\title{
Copper(I)-Catalyzed Intramolecular Asymmetric Double C- Arylation for the Formation of Chiral Spirocyclic Bis-oxindoles
}

Ting Liu, ${ }^{\dagger}$ Jiajie Feng, ${ }^{\ddagger},{ }^{*}$ Chen Chen, ${ }^{\dagger}$ Zhuoji Deng, ${ }^{\dagger}$ Rajendraprasad Kotagiri, ${ }^{\dagger}$ Guangxiong $Z^{2}$ hou, ${ }^{\dagger}$ Xinhao Zhang, ${ }^{\ddagger}, "$ Qian Cai ${ }^{\dagger}$

†International Cooperative Laboratory of Traditional Chinese Medicine Modernization and Innovative Drug Development of Chinese Ministry of Education, College of Pharmacy, Jinan University, No 601 Huangpu Avenue West, Guangzhou, 510632 , China

$\ddagger$ Lab of Computational Chemistry and Drug Design, State Key Laboratory of Chemical Oncogenomics, Peking University Shenzhen Graduate School, Shenzhen 518055, China.

\#Shenzhen Bay Laboratory, Shenzhen, 518055, China.

guangxzh@,Sina.com

zhangxinhao@pku.edu.cn

caiqian@jnu.edu.cn

\section{Supporting Information}

Table of Contents

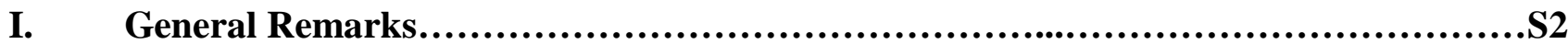

II. Synthesis of Substrates.........................................................S2

III. General Procedure for the Formation of Spirocyclic Bis-oxindoles......................S10

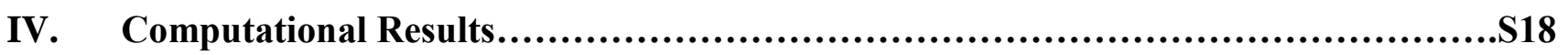

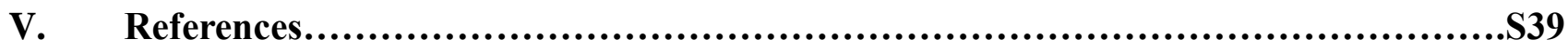

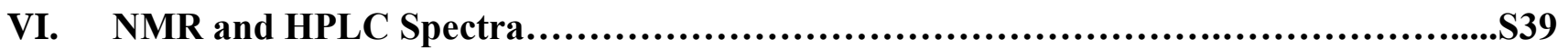




\section{General Remarks}

${ }^{1} \mathrm{H}$ NMR and ${ }^{13} \mathrm{C}$ NMR spectra were recorded on a Bruker AV-400 or $500 \mathrm{MHz}$ spectrometer. Chemical shifts $(\delta)$ are given in relative to tetramethylsilane $(\delta 0.00 \mathrm{ppm})$ in $\mathrm{CDCl}_{3}$. Coupling constants, $J$, were reported in hertz unit (Hz). High resolution mass spectra (HRMS) were obtained on a Q-STAR Elite ESI-LC-MS/MS Spectrometer. Chemical names were generated using Cambridge Soft. ChemDraw Ultra 10.0. Optical rotations were measured on a Perkin Elmer 341 polarimeter. Enantiomeric ratios were determined by chiral HPLC using a chiralpak AD-H (Amylose tris (3,5-dimethylphenylcarbamate)coated on 5 $\mu \mathrm{m}$ silica-gel ), chiralpak AS-H (Amylose tris[(S)- $\alpha$-methylbenzylcarbamate] coated on $5 \mu$ m silica-gel) or chiralcel OD-H column (Cellulose tris (3,5-dimethylphenylcarbamate) coated on $5 \mu \mathrm{m}$ silica-gel) with hexane and $i-\mathrm{PrOH}$ as solvents. Commercially obtained reagents were used without further purification.

\section{Synthesis of Substrates}

A general procedure for the synthesis of $1 \mathrm{a}-\mathrm{m}^{[1,2]}$ :

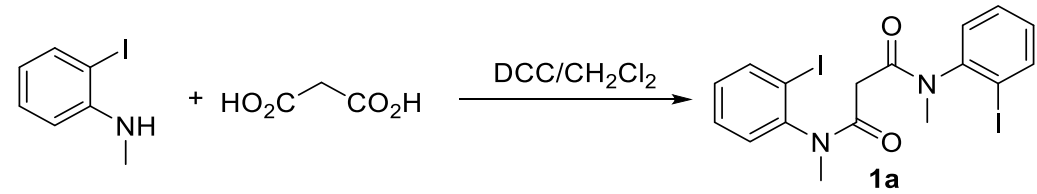

To a solution of $\mathrm{N}$-methyl 2-iodoaniline $(2.33 \mathrm{~g}, 10 \mathrm{mmol})$ in DCM $(50 \mathrm{~mL})$ was added successively malonic acid $(520 \mathrm{mg}, 5 \mathrm{mmol})$ and DCC $(2.27 \mathrm{~g}, 11 \mathrm{mmol})$. The reaction mixture was stirred at room temperature for 24 hours. The mixture was filtrated and the filtrate was evaporated to remove the solvent. The residue was purified through column to afford the corresponding product 1a (1.36 g, 51\% yield). Compounds $\mathbf{1 b}-\mathbf{1 m}$ were synthesized according to similar procedure.

\section{A general procedure for the synthesis of $3 a-i^{[1,2]}$ :}

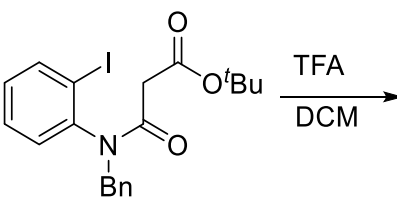

S1<smiles>O=C(O)CC(=O)N(Cc1ccccc1)c1ccccc1I</smiles>

S2
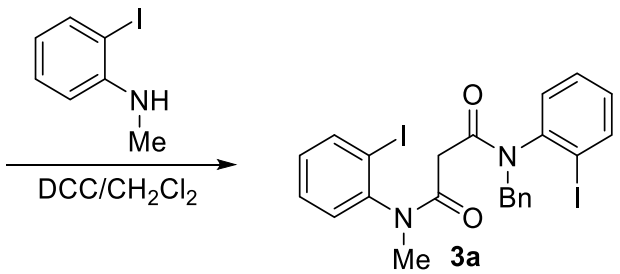

To a solution of $\mathbf{S 1}(9.2 \mathrm{~g}, 20 \mathrm{mmol})$ in DCM $(60 \mathrm{~mL})$ was added TFA $(10 \mathrm{~mL})$. The reaction mixture was stirred at room temperature for 12 hours. The solvent was evaporated. The residue was neutralized with $\mathrm{K}_{2} \mathrm{CO}_{3}$ aqueous solution. The mixture was extracted with EtOAc (20 mL X 3), dried over anhydrous $\mathrm{Na}_{2} \mathrm{SO}_{4}$ and concentrated in vacuo to give $\mathbf{S 2}$, which was directly used in the next step without further purification.

To a solution of $N$-methyl 2-iodoaniline $(1.77 \mathrm{~g}, 7.6 \mathrm{mmol})$ in DCM $(40 \mathrm{~mL})$ was added successively S2 $(3.0 \mathrm{~g}, 7.6 \mathrm{mmol})$ and DCC $(1.57 \mathrm{~g}, 7.6 \mathrm{mmol})$. The reaction mixture was stirred at room temperature for 24 hours. The mixture was filtrated and the filtrate was evaporated to remove the solvent. The residue was purified through column to afford the corresponding product $\mathbf{3 a}$ (3.58 g, 77\% yield). Compounds $\mathbf{3 b - 3 i}$ were synthesized acoording to similar procedures. 
<smiles>CN(C(=O)CC(=O)N(C)c1ccccc1I)c1ccccc1I</smiles>

$1.36 \mathrm{~g}, 51 \%$ yield, white solid, m.p. $152-153{ }^{\circ} \mathrm{C} .{ }^{1} \mathrm{H}$ NMR $\left(400 \mathrm{MHz}, \mathrm{CDCl}_{3}\right.$, two rotamers) $\delta$ $7.71 \& 7.65(2 \mathrm{dd}, J=8.0 \mathrm{~Hz}, 1.2 \mathrm{~Hz}, 2 \mathrm{H}), 7.22-7.26(\mathrm{~m}, 3 \mathrm{H}), 6.87-6.95(\mathrm{~m}, 2 \mathrm{H}), 6.59$ (dd, $J=7.6$ $\mathrm{Hz}, 1.6 \mathrm{~Hz}, 1 \mathrm{H}$ ), 2.93 \& 2.89 (two s, 6H,), 2.73 (d, $J=16.0 \mathrm{~Hz}, 0.5 \mathrm{H}), 2.67$ (s, 1H), 2.58 (d, $J=$ $16.0 \mathrm{~Hz}, 0.5 \mathrm{H}) ;{ }^{13} \mathrm{C}$ NMR $\left(100 \mathrm{MHz}, \mathrm{CDCl}_{3}\right.$, two rotamers) $\delta 166.5,166.2,145.3,145.1,140.0$, $139.9,130.2,129.8,129.7,129.3,99.7,99.2,42.3,41.6,36.2,35.9$; ESI-MS $m / z$ 535.0 (M + H $)^{+}$; HRMS calcd for $\mathrm{C}_{17} \mathrm{H}_{17} \mathrm{I}_{2} \mathrm{~N}_{2} \mathrm{O}_{2}+(\mathrm{M}+\mathrm{H})^{+} 534.9374$, found 534.9366 .

\section{$N^{1}, N^{3}$-diethyl- $N^{1}, N^{3}$-bis(2-iodophenyl)malonamide (1b)}

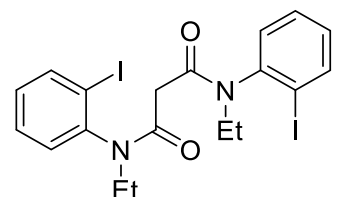

White solid, m.p. $213-214{ }^{\circ} \mathrm{C} .{ }^{1} \mathrm{H}$ NMR $\left(400 \mathrm{MHz}, \mathrm{CDCl}_{3}\right.$, two rotamers) $\delta 7.89 \& 7.83$ (2dd, $J=8.0$ $\mathrm{Hz}, 1.6 \mathrm{~Hz}, 2 \mathrm{H}), 7.37-7.38$ (m, 2H), 7.29-7.30 (m, 1H), 7.07-7.09 (m, 2H), 6.66 (dd, $J=8.0 \mathrm{~Hz}, 1.6$ $\mathrm{Hz}, 1 \mathrm{H}), 4.07-4.15$ (m, 2H), 2.98-3.09 (m, 2H), $2.83(\mathrm{~d}, J=16.0 \mathrm{~Hz}, 0.5 \mathrm{H}), 2.76(\mathrm{~s}, 1 \mathrm{H}), 2.67$ (d, $J$ $=16.0 \mathrm{~Hz}, 0.5 \mathrm{H}), 1.11(\mathrm{t}, J=7.2 \mathrm{~Hz}, 3 \mathrm{H}), 1.05(\mathrm{t}, J=7.2 \mathrm{~Hz}, 3 \mathrm{H}) ;{ }^{13} \mathrm{C} \mathrm{NMR}\left(100 \mathrm{MHz}, \mathrm{CDCl}_{3}\right.$, two rotamers) $\delta 166.5,166.2,143.9,143.8,140.2,140.1,131.2,130.8,130.0,129.9,129.7,129.3$, 100.8, 100.3, 43.6, 43.3, 43.1, 42.3, 12.7; ESI-MS $\mathrm{m} / \mathrm{z} 563.0(\mathrm{M}+\mathrm{H})^{+}$; HRMS calcd for $\mathrm{C}_{19} \mathrm{H}_{21} \mathrm{I}_{2} \mathrm{~N}_{2} \mathrm{O}_{2}{ }^{+}(\mathrm{M}+\mathrm{H})^{+}$562.9687, found 562.9706.

\section{$N^{1}, N^{3}$-diallyl- $N^{1}, N^{3}$-bis(2-iodophenyl)malonamide (1c)}

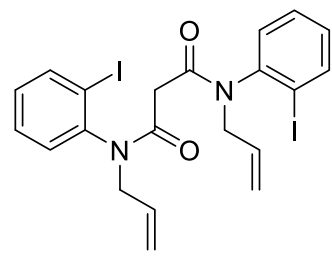

White solid, m.p. $139-140{ }^{\circ} \mathrm{C} .{ }^{1} \mathrm{H}$ NMR $\left(400 \mathrm{MHz}, \mathrm{CDCl}_{3}\right.$, two rotamers) $\delta 7.88 \& 7.82(2 \mathrm{dd}, J=8.0$ $\mathrm{Hz}, 1.2 \mathrm{~Hz}, 2 \mathrm{H}), 7.26-7.36(\mathrm{~m}, 3 \mathrm{H}), 7.00-7.07(\mathrm{~m}, 2 \mathrm{H}), 6.63(\mathrm{dd}, J=7.6 \mathrm{~Hz}, 1.6 \mathrm{~Hz}, 1 \mathrm{H}), 5.78-5.89$ (m, 2H), 5.05-5.10 (m, 4H), 4.73-4.79 (m, 2H), 3.40-3.51 (m, 2H), 2.89 (d, $J=16.0 \mathrm{~Hz}, 0.5 \mathrm{H}), 2.82$ (s, $1 \mathrm{H}), 2.71(\mathrm{~d}, J=16.0 \mathrm{~Hz}, 0.5 \mathrm{H}) ;{ }^{13} \mathrm{C} \mathrm{NMR}\left(100 \mathrm{MHz}, \mathrm{CDCl}_{3}\right.$, two rotamers) $\delta 166.5,166.2$, 143.6 143.5, 140.1, 140.0, 132.1, 131.5, 131.1, 130.2, 130.1, 129.7, 129.2, 118.8, 100.7, 100.3, 51.5, 51.2, 43.1, 42.2; ESI-MS $m / z 587.0(\mathrm{M}+\mathrm{H})^{+}$; HRMS calcd for $\mathrm{C}_{21} \mathrm{H}_{21} \mathrm{I}_{2} \mathrm{~N}_{2} \mathrm{O}_{2}{ }^{+}(\mathrm{M}+\mathrm{H})^{+}$586.9687, found 586.9708 .

\section{$N^{1}, N^{3}$-dibenzyl- $N^{1}, N^{3}$-bis(2-iodophenyl)malonamide (1d)}<smiles>O=C(CC(=O)Nc1ccccc1I)Nc1ccccc1I</smiles> 
White solid, m.p. $176-177^{\circ} \mathrm{C} .{ }^{1} \mathrm{H}$ NMR (400 MHz, $\mathrm{CDCl}_{3}$, two rotamers) $\delta$ 7.82-7.85 (m, 2H), 7.17$7.26(\mathrm{~m}, 11 \mathrm{H}), 6.88-6.99(\mathrm{~m}, 4 \mathrm{H}), 6.15(\mathrm{~d}, J=6.8 \mathrm{~Hz}, 1 \mathrm{H}), 5.60 \& 5.57(2 \mathrm{~d}, J=14.4 \mathrm{~Hz}, 2 \mathrm{H})$, $3.92 \& 3.84(2 \mathrm{~d}, J=14.4 \mathrm{~Hz}, 2 \mathrm{H}), 2.98(\mathrm{~d}, J=16.0 \mathrm{~Hz}, 0.5 \mathrm{H}), 2.91(\mathrm{~s}, 1 \mathrm{H}), 2.73(\mathrm{~d}, J=16.0 \mathrm{~Hz}$, $0.5 \mathrm{H}) ;{ }^{13} \mathrm{C}$ NMR $\left(100 \mathrm{MHz}, \mathrm{CDCl}_{3}\right.$, two rotamers) $\delta 166.8,166.5,143.3,143.2,140.1,140.0,136.4$, 136.3, 131.7, 131.1, 130.1, 129.9, 129.5, 129.3, 129.1, 128.4, 128.3, 127.5, 100.3, 100.0, 51.8, 51.6, 43.1, 42.3; ESI-MS $m / z 687.0(\mathrm{M}+\mathrm{H})^{+}$; HRMS calcd for $\mathrm{C}_{29} \mathrm{H}_{25} \mathrm{I}_{2} \mathrm{~N}_{2} \mathrm{O}_{2}{ }^{+}(\mathrm{M}+\mathrm{H})^{+} 687.0000$, found 686.9993.

\section{$N^{1}, N^{3}$-dibenzyl- $N^{1}, N^{3}$-bis(2-iodo-4-methylphenyl)malonamide (1e)}<smiles>Cc1ccc(NC(=O)CC(=O)N(C(=O)c2ccccc2)c2ccc(C)cc2I)c(I)c1</smiles>

White solid, m.p. $210-21{ }^{\circ} \mathrm{C} .{ }^{1} \mathrm{H}$ NMR (400 MHz, $\mathrm{CDCl}_{3}$, two rotamers) $\delta 7.66 \& 7.64(2 \mathrm{~d}, J=1.2$ $\mathrm{Hz}, 2 \mathrm{H}), 7.25-7.27(\mathrm{~m}, 11 \mathrm{H}), 6.96(\mathrm{dd}, J=8.0 \mathrm{~Hz}, 1.2 \mathrm{~Hz}, 1 \mathrm{H}), 6.81(\mathrm{~d}, J=8.0 \mathrm{~Hz}, 1 \mathrm{H}), 6.66(\mathrm{dd}$, $J=8.0 \mathrm{~Hz}, 1.2 \mathrm{~Hz}, 1 \mathrm{H}), 6.00(\mathrm{~d}, J=8.0 \mathrm{~Hz}, 1 \mathrm{H}), 5.61 \& 5.57(2 \mathrm{~d}, J=16.0 \mathrm{~Hz}, 2 \mathrm{H}), 3.89 \& 3.81(2 \mathrm{dd}$, $J=14.4 \mathrm{~Hz}, 2 \mathrm{H}), 2.97(\mathrm{~d}, J=16.0 \mathrm{~Hz}, 0.5 \mathrm{H}), 2.91(\mathrm{~s}, 1 \mathrm{H}), 2.77(\mathrm{~d}, J=16.0 \mathrm{~Hz}, 0.5 \mathrm{H}), 2.25 \& 2.23$ $(2 \mathrm{~s}, 6 \mathrm{H}),{ }^{13} \mathrm{C} \mathrm{NMR}\left(100 \mathrm{MHz}, \mathrm{CDCl}_{3}\right.$, two rotamers) $\delta 167.1,166.8,140.7,140.6,140.4,140.3$, 140.2, 136.5, 136.4, 131.1, 130.6, 130.2, 129.8, 129.5, 129.2, 128.4, 128.3, 127.5, 127.4, 100.0, 99.6, 51.8, 51.6, 43.1, 42.2, 20.6, 20.5; ESI-MS $m / z$ 715.0 (M + H) ${ }^{+}$; HRMS calcd for $\mathrm{C}_{31} \mathrm{H}_{29} \mathrm{I}_{2} \mathrm{~N}_{2} \mathrm{O}_{2}{ }^{+}$ $(\mathrm{M}+\mathrm{H})^{+} 715.0313$, found 715.0328 .

\section{$N^{1}, N^{3}$-dibenzyl- $N^{1}, N^{3}$-bis(2-iodo-3-methylphenyl)malonamide (1f)}<smiles>Cc1cccc(N(Br)C(=O)CC(=O)N(Cc2ccccc2)c2cccc(C)c2I)c1I</smiles>

White solid, m.p. $142-143{ }^{\circ} \mathrm{C} .{ }^{1} \mathrm{H}$ NMR $\left(400 \mathrm{MHz}, \mathrm{CDCl}_{3}\right.$, two rotamers) $\delta$ 7.20-7.27 (m, 10H), 7.02-7.13 (m, 3H), 6.68-6.74 (m, 2H), 5.87 (dd, $J=7.6 \mathrm{~Hz}, 0.8 \mathrm{~Hz}, 1 \mathrm{H}), 5.64 \& 5.62(2 \mathrm{~d}, J=14.4$ $\mathrm{Hz}, 2 \mathrm{H}), 3.84 \& 3.77(2 \mathrm{~d}, J=14.4 \mathrm{~Hz}, 2 \mathrm{H}), 2.96(\mathrm{~d}, J=16.0 \mathrm{~Hz}, 0.5 \mathrm{H}), 2.92(\mathrm{~s}, 1 \mathrm{H}), 2.75$ (d, $J=$ $16.0 \mathrm{~Hz}, 0.5 \mathrm{H}$ ), 2.46\&2.45 (two s, 6H); ${ }^{13} \mathrm{C} \mathrm{NMR}\left(100 \mathrm{MHz}, \mathrm{CDCl}_{3}\right.$, two rotamers) $\delta 167.0,166.7$, 144.3, 144.1, 143.7, 143.6, 136.6, 136.5, 129.6, 129.5, 129.3, 129.2, 128.8, 128.7, 128.4, 128.3, 128.1, 127.4, 107.4, 107.0, 51.8, 51.6, 43.0, 42.3, 29.6, 29.5; ESI-MS m/z 715.0 (M + H $)^{+}$; HRMS calcd for $\mathrm{C}_{31} \mathrm{H}_{29} \mathrm{I}_{2} \mathrm{~N}_{2} \mathrm{O}_{2}{ }^{+}(\mathrm{M}+\mathrm{H})^{+} 715.0313$, found 715.0298.

\section{$N^{1}, N^{3}$-dibenzyl- $N^{1}, N^{3}$-bis(2-iodo-4-methoxyphenyl)malonamide (1g)}<smiles>COc1ccc(N(Br)C(=O)CC(=O)N(Cc2ccccc2)c2ccc(OC)cc2I)c(I)c1</smiles>

Light yellow solid, m.p. $145-146{ }^{\circ} \mathrm{C} .{ }^{1} \mathrm{H}$ NMR $\left(400 \mathrm{MHz}, \mathrm{CDCl}_{3}\right.$, two rotamers) $\delta$ 7.64-7.66 (m, 2H), 7.20-7.33 (m, 10H), $6.82(\mathrm{~d}, J=8.8 \mathrm{~Hz}, 1 \mathrm{H}), 6.68(\mathrm{dd}, J=8.8 \mathrm{~Hz}, 2.8 \mathrm{~Hz}, 1 \mathrm{H}), 6.37(\mathrm{dd}, J=$ 
$8.8 \mathrm{~Hz}, 2.8 \mathrm{~Hz}, 1 \mathrm{H}), 6.06(\mathrm{~d}, J=8.4 \mathrm{~Hz}, 1 \mathrm{H}), 5.59 \& 5.58(2 \mathrm{~d}, J=14.4 \mathrm{~Hz}, 2 \mathrm{H}), 3.87 \& 3.79(2 \mathrm{~d}, J=$ $14.4 \mathrm{~Hz}, 2 \mathrm{H}$ ), $3.74 \& 3.71$ (two s, 6H), 2.97 (d, $J=16.0 \mathrm{~Hz}, 0.5 \mathrm{H}$ ), 2.92 (s, $1 \mathrm{H}$ ), 2.78 (d, $J=16.0 \mathrm{~Hz}$, $0.5 \mathrm{H}) ;{ }^{13} \mathrm{C}$ NMR $\left(100 \mathrm{MHz}, \mathrm{CDCl}_{3}\right.$, two rotamers) $\delta 167.4,167.2,159.5,159.3,136.6,136.5,136.2$, 136.1, 131.8, 131.1, 129.5, 129.3, 128.4, 128.3, 127.5, 127.4, 124.9, 124.8, 114.8, 114.2, 100.5, 100.1, 55.8, 55.7, 51.9, 51.8, 42.9, 42.1; ESI-MS $\mathrm{m} / \mathrm{z} 747.0(\mathrm{M}+\mathrm{H})^{+}$; HRMS calcd for $\mathrm{C}_{31} \mathrm{H}_{28} \mathrm{I}_{2} \mathrm{~N}_{2} \mathrm{NaO}_{4}{ }^{+}(\mathrm{M}+\mathrm{Na})^{+} 769.0031$, found 769.0029 .

\section{$N^{1}, N^{3}$-dibenzyl- $N^{1}, N^{3}$-bis(4-bromo-2-iodophenyl)malonamide (1h)}<smiles>O=C(CC(=O)N(Cc1ccccc1)c1ccc(Br)cc1I)N(Br)c1ccc(Br)cc1I</smiles>

Light yellow solid, m.p. $118-119{ }^{\circ} \mathrm{C} .{ }^{1} \mathrm{H}$ NMR $\left(400 \mathrm{MHz}, \mathrm{CDCl}_{3}\right.$, two rotamers) $\delta$ 7.98-8.00 (m, 2H), 7.17-7.31 (m, 11H), 6.98-7.01 (m, 1H), $6.78(\mathrm{~d}, J=8.4 \mathrm{~Hz}, 1 \mathrm{H}), 6.04(\mathrm{~d}, J=8.4 \mathrm{~Hz}, 1 \mathrm{H})$, $5.59 \& 5.56(2 \mathrm{~d}, J=14.4 \mathrm{~Hz}, 2 \mathrm{H}), 3.86 \& 3.80(2 \mathrm{~d}, J=14.4 \mathrm{~Hz}, 2 \mathrm{H}), 2.98(\mathrm{~d}, J=16.0 \mathrm{~Hz}, 0.5 \mathrm{H})$, $2.85(\mathrm{~s}, 1 \mathrm{H}), 2.68(\mathrm{~d}, J=16.0 \mathrm{~Hz}, 0.5 \mathrm{H}) ;{ }^{13} \mathrm{C}$ NMR $\left(100 \mathrm{MHz}, \mathrm{CDCl}_{3}\right.$, two rotamers $) \delta 166.3,166.0$, $142.4,142.3,142.1,142.0,136.0,135.9,132.7,132.6,132.3,131.9,129.5,129.3,128.6,128.5$, 127.9, 127.8, 123.2, 123.0, 101.3, 100.9, 51.7, 51.6, 42.9, 42.4; ESI-MS $m / z$ 842.8 (M+ H $)^{+}$; HRMS calcd for $\mathrm{C}_{29} \mathrm{H}_{22} \mathrm{Br}_{2} \mathrm{I}_{2} \mathrm{~N}_{2} \mathrm{NaO}_{2}{ }^{+}(\mathrm{M}+\mathrm{Na})^{+}$864.8030, found 864.8027.

$N^{1}, N^{3}$-dibenzyl- $N^{1}, N^{3}$-bis(4-chloro-2-iodophenyl)malonamide (1i)<smiles>O=C(CC(=O)N(Cc1ccccc1)c1ccc(Cl)cc1I)N(Br)c1ccc(Cl)cc1I</smiles>

White solid, m.p. $123-124{ }^{\circ} \mathrm{C} .{ }^{1} \mathrm{H}$ NMR $\left(400 \mathrm{MHz}, \mathrm{CDCl}_{3}\right.$, two rotamers) $\delta$ 7.83-7.85 (m, 2H), 7.17$7.29(\mathrm{~m}, 11 \mathrm{H}), 6.89(\mathrm{dd}, J=8.4 \mathrm{~Hz}, 2.4 \mathrm{~Hz}, 1 \mathrm{H}), 6.84(\mathrm{~d}, J=8.4 \mathrm{~Hz}, 1 \mathrm{H}), 6.12(\mathrm{~d}, J=8.4 \mathrm{~Hz}, 1 \mathrm{H})$, $5.60 \& 5.56(2 \mathrm{~d}, J=14.0 \mathrm{~Hz}, 2 \mathrm{H}), 3.87 \& 3.82(2 \mathrm{~d}, J=14.4 \mathrm{~Hz}, 2 \mathrm{H}), 2.98(\mathrm{~d}, J=16.0 \mathrm{~Hz}, 0.5 \mathrm{H})$, $2.86(\mathrm{~s}, 1 \mathrm{H}), 2.69(\mathrm{~d}, J=16.0 \mathrm{~Hz}, 0.5 \mathrm{H}) ;{ }^{13} \mathrm{C} \mathrm{NMR}\left(100 \mathrm{MHz}, \mathrm{CDCl}_{3}\right.$, two rotamers $) \delta 166.4,166.1$, $141.9,141.8,139.4,139.3,136.0,135.9,135.1,135.0,132.2$, 131.5, 129.6, 129.4, 129.3, 128.6, 128.5, 127.9, 127.8, 100.8, 100.3, 51.7, 51.6, 42.9, 42.3; ESI-MS $m / z$ 754.9 (M + H $)^{+}$; HRMS calcd for $\mathrm{C}_{29} \mathrm{H}_{23} \mathrm{Cl}_{2} \mathrm{I}_{2} \mathrm{~N}_{2} \mathrm{O}_{2}{ }^{+}(\mathrm{M}+\mathrm{H})^{+} 754.9221$, found 754.9243 .

Dimethyl 4,4'-(malonylbis(benzylazanediyl))bis(3-iodobenzoate) (1j)<smiles>CC(=O)c1ccc(N(Cc2ccccc2)C(=O)CC(=O)N(Cc2ccccc2)c2ccc(C(C)=O)cc2I)c(I)c1</smiles>

White solid, m.p. $161-162{ }^{\circ} \mathrm{C} .{ }^{1} \mathrm{H}$ NMR (400 MHz, $\mathrm{CDCl}_{3}$, two rotamers) $\delta 8.49 \& 8.46(2 \mathrm{~d}, J=1.6$ $\mathrm{Hz}, 2.0 \mathrm{~Hz}, 2 \mathrm{H}), 7.73(\mathrm{~d}, J=8.4 \mathrm{~Hz}, 1 \mathrm{H}), 7.50(\mathrm{~d}, J=8.0 \mathrm{~Hz}, 1 \mathrm{H}), 7.06-7.15(\mathrm{~m}, 10 \mathrm{H}), 6.92$ (d, $J$ $=8.0 \mathrm{~Hz}, 1 \mathrm{H}), 6.14(\mathrm{~d}, J=8.0 \mathrm{~Hz}, 1 \mathrm{H}), 5.59 \& 5.56(2 \mathrm{~d}, J=14.0 \mathrm{~Hz}, 2 \mathrm{H}), 3.92 \& 3.84(2 \mathrm{~d}, J=14.4$ 
$\mathrm{Hz}, 2 \mathrm{H}), 3.90 \& 3.87(2 \mathrm{~s}, 6 \mathrm{H}), 2.97(\mathrm{~d}, J=16.0 \mathrm{~Hz}, 0.5 \mathrm{H}), 2.83(\mathrm{~s}, 1 \mathrm{H}), 2.67(\mathrm{~d}, J=16.0 \mathrm{~Hz}, 0.5 \mathrm{H})$; ${ }^{13} \mathrm{C}$ NMR (100 MHz, $\mathrm{CDCl}_{3}$, two rotamers) $\delta 165.9,165.6,164.5,164.3,147.0,146.9,141.1,141.0$, 135.8, 135.7, 131.6, 131.5, 130.8, 130.5, 130.2, 129.3, 129.2, 128.5, 128.4, 127.8, 100.3, 99.9, 52.8, 52.7, 51.6, 51.4, 43.1, 42.2; ESI-MS m/z $803.0(\mathrm{M}+\mathrm{H})^{+} ; \mathrm{HRMS}$ calcd for $\mathrm{C}_{33} \mathrm{H}_{29} \mathrm{I}_{2} \mathrm{~N}_{2} \mathrm{O}_{4}{ }^{+}(\mathrm{M}+\mathrm{H})^{+}$ 803.0110, found 803.0125.

$N^{1}, N^{3}$-dibenzyl- $N^{1}, N^{3}$-bis(4-acetyl-2-iodophenyl)malonamide (1k)<smiles>CC(=O)c1ccc(NC(=O)CC(=O)N(Cc2ccccc2)c2ccc(C(C)=O)cc2I)c(I)c1</smiles>

White solid, m.p. $135-136{ }^{\circ} \mathrm{C} .{ }^{1} \mathrm{H}$ NMR (400 MHz, $\mathrm{CDCl}_{3}$, two rotamers) $\delta 8.39 \& 8.36(2 \mathrm{~d}, J=2.0$ $\mathrm{Hz}, 2 \mathrm{H}), 7.73 \& 7.42(2 \mathrm{dd}, J=8.0,2.0 \mathrm{~Hz}, 2 \mathrm{H}), 7.16-7.26(\mathrm{~m}, 10 \mathrm{H}), 7.05 \& 6.18(2 \mathrm{~d}, J=8.0 \mathrm{~Hz}, 2 \mathrm{H})$, $5.61 \& 5.60(2 \mathrm{~d}, J=14.4 \mathrm{~Hz}, 2 \mathrm{H}), 3.94 \& 3.84(2 \mathrm{~d}, J=14.4 \mathrm{~Hz}, 2 \mathrm{H}), 2.99(\mathrm{~d}, J=16.0 \mathrm{~Hz}, 0.5 \mathrm{H})$, $2.86(\mathrm{~s}, 1 \mathrm{H}), 2.66(\mathrm{~d}, J=16.0 \mathrm{~Hz}, 0.5 \mathrm{H}), 2.53 \& 2.51(2 \mathrm{~s}, 6 \mathrm{H}) ;{ }^{13} \mathrm{C} \mathrm{NMR}\left(100 \mathrm{MHz}, \mathrm{CDCl}_{3}\right.$, two rotamers) $\delta 195.7,195.6,165.9,165.6,147.1,147.0,140.0,139.9,138.1,137.9,135.9,135.7,131.8$, 131.0, 129.5, 129.3, 129.2, 128.9, 128.6, 128.5, 127.9, 100.9, 100.5, 51.7, 51.5, 43.0, 42.2, 26.8; ESI-MS $\mathrm{m} / z$ 793.0 $(\mathrm{M}+\mathrm{Na})^{+}$; HRMS calcd for $\mathrm{C}_{33} \mathrm{H}_{28} \mathrm{I}_{2} \mathrm{~N}_{2} \mathrm{NaO}_{4}{ }^{+}(\mathrm{M}+\mathrm{Na})^{+} 793.0031$, found 793.0019 .

$N^{1}, N^{3}$-dibenzyl- $N^{1}, N^{3}$-bis(2-iodo-5-methylphenyl)malonamide (11)<smiles>Cc1ccc(I)c(NC(=O)CC(=O)N(Cc2ccccc2)c2cc(C)ccc2I)c1</smiles>

White solid, m.p. $135-136{ }^{\circ} \mathrm{C} .{ }^{1} \mathrm{H}$ NMR (400 MHz, $\mathrm{CDCl}_{3}$, two rotamers) $\delta 7.71 \& 7.68(2 \mathrm{~d}, J=8.0$ $\mathrm{Hz}, 2 \mathrm{H}), 7.27-7.29(\mathrm{~m}, 8 \mathrm{H}), 7.23-7.26(\mathrm{~m}, 2 \mathrm{H}), 6.77-6.84(\mathrm{~m}, 3 \mathrm{H}), 6.16(\mathrm{~d}, J=1.6 \mathrm{~Hz}, 1 \mathrm{H})$, $5.54 \& 5.48(2 \mathrm{~d}, J=14.4 \mathrm{~Hz}, 2 \mathrm{H}), 4.03 \& 4.02(2 \mathrm{~d}, J=14.4 \mathrm{~Hz}, 2 \mathrm{H}), 3.02(\mathrm{~d}, J=16.4 \mathrm{~Hz}, 0.5 \mathrm{H})$, $2.94(\mathrm{~s}, 1 \mathrm{H}), 2.87(\mathrm{~d}, J=16.4 \mathrm{~Hz}, 0.5 \mathrm{H}), 2.16 \& 2.06(2 \mathrm{~s}, 6 \mathrm{H}) ;{ }^{13} \mathrm{C} \mathrm{NMR}\left(100 \mathrm{MHz}, \mathrm{CDCl}_{3}\right.$, two rotamers) $\delta 166.9,166.6,143.2,143.1,139.9,139.6,139.5,139.4,136.5,136.4,132.3,131.7,131.0$, 130.8, 129.4, 129.2, 128.4, 128.3, 127.4, 95.9, 95.4, 51.9, 51.7, 43.0, 42.5, 20.8, 20.7; ESI-MS m/z $715.0(\mathrm{M}+\mathrm{H})^{+} ;$HRMS calcd for $\mathrm{C}_{31} \mathrm{H}_{29} \mathrm{I}_{2} \mathrm{~N}_{2} \mathrm{O}_{2}{ }^{+}(\mathrm{M}+\mathrm{H})^{+} 715.0313$, found 715.0312.

\section{$N^{1}, N^{3}$-dibenzyl- $N^{1}, N^{3}$-bis(5-chloro-2-iodophenyl)malonamide (1m)}<smiles>O=C(CC(=O)N(Cc1ccccc1)c1cc(Cl)ccc1I)Nc1cc(Cl)ccc1I</smiles>

White solid, m.p. $143-144^{\circ} \mathrm{C} .{ }^{1} \mathrm{H}$ NMR (400 MHz, $\mathrm{CDCl}_{3}$, two rotamers) $\delta 7.80 \& 7.75(2 \mathrm{~d}, J=8.4$ $\mathrm{Hz}, 2 \mathrm{H}), 7.28-7.33(\mathrm{~m}, 8 \mathrm{H}), 7.18-7.20(\mathrm{~m}, 2 \mathrm{H}), 6.99-7.02(\mathrm{~m}, 3 \mathrm{H}), 6.38(\mathrm{~d}, J=1.6 \mathrm{~Hz}, 1 \mathrm{H})$, $5.54 \& 5.48(2 \mathrm{~d}, J=14.4 \mathrm{~Hz}, 2 \mathrm{H}), 4.09 \& 4.06(2 \mathrm{~d}, J=14.4 \mathrm{~Hz}, 2 \mathrm{H}), 3.08(\mathrm{~d}, J=16.0 \mathrm{~Hz}, 0.5 \mathrm{H})$, 
$2.91(\mathrm{~s}, 1 \mathrm{H}), 2.81(\mathrm{~d}, J=16.0 \mathrm{~Hz}, 0.5 \mathrm{H}) ;{ }^{13} \mathrm{C} \mathrm{NMR}\left(100 \mathrm{MHz}, \mathrm{CDCl}_{3}\right.$, two rotamers) $\delta 166.2,165.9$, 144.4, 144.2, 140.8, 140.6, 135.7, 135.6, 135.3, 134.8, 131.8, 131.1, 130.5, 130.4, 129.3, 129.2, 128.6, 127.8, 97.8, 97.4, 51.9, 51.6, 43.1, 42.6; ESI-MS $\mathrm{m} / \mathrm{z} 755.0(\mathrm{M}+\mathrm{H})^{+}$; HRMS calcd for $\mathrm{C}_{29} \mathrm{H}_{23} \mathrm{Cl}_{2} \mathrm{I}_{2} \mathrm{~N}_{2} \mathrm{O}_{2}{ }^{+}(\mathrm{M}+\mathrm{H})^{+} 754.9220$, found 754.9209 .

$N^{I}$-benzyl- $N^{I}, N^{3}$-bis(2-iodophenyl)- $N^{3}$-methylmalonamide (3a)

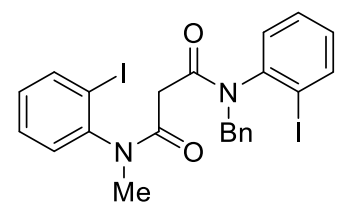

White solid, m.p. 197-198 ${ }^{\circ} \mathrm{C} .{ }^{1} \mathrm{H}$ NMR $\left(400 \mathrm{MHz}, \mathrm{CDCl}_{3}\right) \delta 7.91 \& 7.89(2 \mathrm{dd}, J=7.6 \mathrm{~Hz}, 1.2 \mathrm{~Hz}$, 2H), 7.40-7.51 (m, 1H), 7.217.28 (m, 5H), 6.96-7.08 (m, 4H), 6.75\&6.08 (2dd, $J=7.6 \mathrm{~Hz}, 1.6 \mathrm{~Hz}$, $1 \mathrm{H}), 5.62 \& 5.58(2 \mathrm{~d}, J=14.4 \mathrm{~Hz}, 1 \mathrm{H}), 3.86 \& 3.81(2 \mathrm{~d}, J=14.4 \mathrm{~Hz}, 1 \mathrm{H}), 3.15 \& 3.09$ (two s, $3 \mathrm{H})$, $2.94(\mathrm{~d}, J=15.6 \mathrm{~Hz}, 0.5 \mathrm{H}), 2.90(\mathrm{~d}, J=16.0 \mathrm{~Hz}, 0.5 \mathrm{H}), 2.85(\mathrm{~d}, J=15.6 \mathrm{~Hz}, 0.5 \mathrm{H}), 2.72(\mathrm{~d}, J=$ $16.0 \mathrm{~Hz}, 0.5 \mathrm{H}) ;{ }^{13} \mathrm{C} \mathrm{NMR}\left(100 \mathrm{MHz}, \mathrm{CDCl}_{3}\right) \delta 166.9,166.8,166.5,166.4,145.6,145.4,143.4$, $140.2,140.1,140.0,139.9,136.4,136.3,131.6,130.3,130.2,130.0,129.9,129.8,129.6,129.5$, $129.2,128.4,128.3,127.6,127.5,100.3,99.9,99.6,99.2,51.8,51.7,42.7,42.0,36.3,36.0$; ESIMS $m / z 611.0(\mathrm{M}+\mathrm{H})^{+}$; HRMS calcd for $\mathrm{C}_{23} \mathrm{H}_{21} \mathrm{I}_{2} \mathrm{~N}_{2} \mathrm{O}_{2}{ }^{+}(\mathrm{M}+\mathrm{H})^{+} 610.9687$, found 610.9674 .

$N^{1}$-allyl- $N^{3}$-benzyl- $N^{1}, N^{3}$-bis(2-iodophenyl)malonamide (3b)

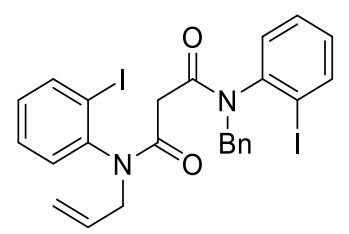

White solid, m.p. $139-140{ }^{\circ} \mathrm{C} .{ }^{1} \mathrm{H}$ NMR $\left(400 \mathrm{MHz}, \mathrm{CDCl}_{3}\right) \delta$ 7.83-7.92 (m, 2H), 7.38-7.40 (m, 1H), 7.23-7.28 (m, 5H), 6.92-7.08 (m, 4H), 6.62\&6.16 (2dd, $J=7.6 \mathrm{~Hz}, 1.6 \mathrm{~Hz}, 1 \mathrm{H}), 5.76-5.97(\mathrm{~m}, 1 \mathrm{H})$, $5.62 \& 5.59(2 \mathrm{~d}, J=14.4 \mathrm{~Hz}, 1 \mathrm{H}), 5.07-5.14(\mathrm{~m}, 2 \mathrm{H}), 4.77-4.82(\mathrm{~m}, 1 \mathrm{H}), 3.90 \& 3.84(2 \mathrm{~d}, J=14.4$ $\mathrm{Hz}, 1 \mathrm{H}), 3.48-3.58(\mathrm{~m}, 1 \mathrm{H}), 2.96(\mathrm{~d}, J=16.0 \mathrm{~Hz}, 0.5 \mathrm{H}), 2.89 \& 2.88(2 \mathrm{~s}, 1 \mathrm{H}), 2.74(\mathrm{~d}, J=16.0 \mathrm{~Hz}$, $0.5 \mathrm{H}) ;{ }^{13} \mathrm{C} \mathrm{NMR}\left(100 \mathrm{MHz}, \mathrm{CDCl}_{3}\right) \delta 166.8,166.5,166.4,166.2,143.6,143.4,143.3,140.1,140.0$, 136.4, 136.3, 132.2, 132.1, 131.6, 131.5, 131.2, 131.0, 130.2, 130.0, 129.9, 129.6, 129.5, 129.4, 129.3, 129.2, 129.1, 128.4, 128.3, 127.6, 127.5, 118.8, 118.7, 100.6, 100.4, 100.3, 99.9, 51.8, 51.7, 51.5, 51.2, 43.1, 42.3; ESI-MS m/z $637.0(\mathrm{M}+\mathrm{H})^{+}$; HRMS calcd for $\mathrm{C}_{25} \mathrm{H}_{23} \mathrm{I}_{2} \mathrm{~N}_{2} \mathrm{O}_{2}{ }^{+}(\mathrm{M}+\mathrm{H})^{+}$ 636.9843 , found 636.9844 .

$N^{1}, N^{3}$-dibenzyl- $N^{1}$-(2-iodo-4-methylphenyl)- $N^{3}$-(2-iodophenyl)malonamide (3c)<smiles>Cc1ccc(N(Br)C(=O)CC(=O)N(Cc2ccccc2)c2ccccc2I)c(I)c1</smiles>

White solid, m.p. 80-81 ${ }^{\circ} \mathrm{C} .{ }^{1} \mathrm{H}$ NMR $\left(400 \mathrm{MHz}, \mathrm{CDCl}_{3}\right) \delta$ 7.83-7.86 (m, 1H), 7.66\&7.64 (2d, $J=$ $1.2 \mathrm{~Hz}, 1 \mathrm{H}), 7.18-7.26(\mathrm{~m}, 10 \mathrm{H}), 6.89-7.00(\mathrm{~m}, 3 \mathrm{H}), 6.78 \& 6.00(2 \mathrm{~d}, J=8.0 \mathrm{~Hz}, 1 \mathrm{H}), 6.66 \& 6.14$ (2dd, $J=8.0 \mathrm{~Hz}, 1.2 \mathrm{~Hz}, 1 \mathrm{H}), 5.54-5.64(\mathrm{~m}, 2 \mathrm{H}), 3.80-3.94(\mathrm{~m}, 2 \mathrm{H}), 2.97(\mathrm{~d}, J=16.0 \mathrm{~Hz}, 0.5 \mathrm{H})$, 
$2.91 \& 2.90(2 \mathrm{~s}, 1 \mathrm{H}), 2.75(\mathrm{~d}, J=16.0 \mathrm{~Hz}, 0.5 \mathrm{H}), 2.26 \& 2.23(2 \mathrm{~s}, 3 \mathrm{H}) ;{ }^{13} \mathrm{C} \mathrm{NMR}\left(100 \mathrm{MHz}, \mathrm{CDCl}_{3}\right)$ $\delta 167.0,166.9,166.7,166.6,143.4,143.3,140.7,140.6,140.4,140.3,140.2,140.1,140.0$, $136.5,136.4,136.3,131.8,131.2,131.0,130.5,130.2,129.9,129.8,129.5,129.4,129.3,129.2$, 128.5, 128.4, 128.3, 127.5, 127.4, 100.3, 100.0, 99.6, 51.9, 51.8, 51.7, 51.6, 43.1, 42.2, 20.6, 20.5; ESI-MS $m / z 701.0(\mathrm{M}+\mathrm{H})^{+} ;$HRMS calcd for $\mathrm{C}_{30} \mathrm{H}_{27} \mathrm{I}_{2} \mathrm{~N}_{2} \mathrm{O}_{2}{ }^{+}(\mathrm{M}+\mathrm{H})^{+}$701.0156, found 701.0157.

\section{$N^{1}, N^{3}$-dibenzyl- $N^{1}$-(2-iodo-4-methoxyphenyl)- $N^{3}$-(2-iodophenyl)malonamide (3d)}<smiles>COc1ccc(N(Cc2ccccc2)C(=O)CC(=O)N(Cc2ccccc2)c2ccccc2I)c(I)c1</smiles>

Light yellow solid, m.p. $157-158{ }^{\circ} \mathrm{C} .{ }^{1} \mathrm{H}$ NMR $\left(400 \mathrm{MHz}, \mathrm{CDCl}_{3}\right) \delta$ 7.82-7.86 (m, 2H), 7.25-7.28 (m, 10.5H), 6.92-6.98 (m, 2H), 6.66-6.68 (m, 0.5H), 6.34-6.37 (m, 0.5H), 6.16-6.19 (m, 0.5H), 6.01 $(\mathrm{d}, J=8.8 \mathrm{~Hz}, 0.5 \mathrm{H}), 5.54-5.64(\mathrm{~m}, 2 \mathrm{H}), 3.81-3.94(\mathrm{~m}, 2 \mathrm{H}), 3.72 \& 3.69(2 \mathrm{~s}, 3 \mathrm{H}), 2.98(\mathrm{~d}, J=16.0$ $\mathrm{Hz}, 0.5 \mathrm{H}), 2.95(\mathrm{~d}, J=16.0 \mathrm{~Hz}, 0.5 \mathrm{H}), 2.89(\mathrm{~d}, J=16.0 \mathrm{~Hz}, 0.5 \mathrm{H}), 2.76(\mathrm{~d}, J=16.0 \mathrm{~Hz}, 0.5 \mathrm{H}) ;{ }^{13} \mathrm{C}$ $\operatorname{NMR}\left(100 \mathrm{MHz}, \mathrm{CDCl}_{3}\right) \delta 167.3,167.1,167.0,166.7,159.5,159.4,143.4,143.3,140.1,140.0$, 136.5, 136.4, 136.3, 136.1, 136.0, 131.8, 131.6, 131.1, 131.0, 130.2, 130.0, 129.5, 129.4, 129.3, $129.2,128.5,128.4,128.3,127.6,127.5,114.8,114.2,100.5,100.3,100.1,100.0,55.8,55.7,51.9$, 51.8, 51.7, 51.6, 43.0, 42.3; ESI-MS $m / 2717.0(\mathrm{M}+\mathrm{H})^{+} ;$HRMS calcd for $\mathrm{C}_{30} \mathrm{H}_{27} \mathrm{I}_{2} \mathrm{~N}_{2} \mathrm{O}_{3}{ }^{+}(\mathrm{M}+\mathrm{H})^{+}$ 717.0106, found 717.0104.

$N^{1}, N^{3}$-dibenzyl- $N^{1}$-(4-chloro-2-iodophenyl)- $N^{3}$-(2-iodophenyl)malonamide (3e)<smiles>O=C(CC(=O)N(Br)c1ccc(Cl)cc1I)c1ccccc1I</smiles>

Light yellow solid, m.p. $130-131^{\circ} \mathrm{C} .{ }^{1} \mathrm{H}$ NMR $\left(400 \mathrm{MHz}, \mathrm{CDCl}_{3}\right) \delta$ 7.79-7.85 (m, 2H), 7.12-7.29 $(\mathrm{m}, 11 \mathrm{H}), 6.78-7.00(\mathrm{~m}, 3 \mathrm{H}), 6.15-6.17(\mathrm{~m}, 0.5 \mathrm{H}), 6.08(\mathrm{~d}, J=8.4 \mathrm{~Hz}, 0.5 \mathrm{H}), 5.55-5.63(\mathrm{~m}, 2 \mathrm{H})$, 3.79-3.91 (m, 2H), $2.99(\mathrm{~d}, J=16.0 \mathrm{~Hz}, 0.5 \mathrm{H}), 2.93(\mathrm{~d}, J=16.0 \mathrm{~Hz}, 0.5 \mathrm{H}), 2.85(\mathrm{~d}, J=16.0 \mathrm{~Hz}$, $0.5 \mathrm{H}), 2.72(\mathrm{~d}, J=16.0 \mathrm{~Hz}, 0.5 \mathrm{H}) ;{ }^{13} \mathrm{C} \mathrm{NMR}\left(100 \mathrm{MHz}, \mathrm{CDCl}_{3}\right) \delta 166.6,166.5,166.4,166.3,143.2$, $143.1,142.0,141.9,140.2,140.1,139.3,136.3,136.2,136.1,136.0,135.1,135.0,132.3,131.7$, $131.6,130.9,130.3,130.1,129.6,129.5,129.4,129.3,129.2,128.6,128.5,128.4,127.8,127.7$, 127.6, 100.9, 100.6, 100.4, 99.8, 51.8, 51.7, 51.7, 51.6, 43.1, 42.4; ESI-MS m/z $721.0(\mathrm{M}+\mathrm{H})^{+}$; HRMS calcd for $\mathrm{C}_{29} \mathrm{H}_{24} \mathrm{ClI}_{2} \mathrm{~N}_{2} \mathrm{O}_{2}{ }^{+}(\mathrm{M}+\mathrm{H})^{+} 720.9610$, found 720.9624 .

$N^{1}, N^{3}$-dibenzyl- $N^{l}$-(4-fluoro-2-iodophenyl)- $N^{3}$-(2-iodophenyl)malonamide (3f)<smiles>O=C(CC(=O)N(Cc1ccccc1)c1ccccc1I)N(Br)c1ccc(F)cc1I</smiles>

Light yellow solid, m.p. $99-100{ }^{\circ} \mathrm{C} .{ }^{1} \mathrm{H}$ NMR $\left(400 \mathrm{MHz}, \mathrm{CDCl}_{3}\right) \delta$ 7.83-7.87 (m, 1H), 7.53-7.55 (m, 
1H), 7.17-7.26 (m, 10.5H), 6.91-6.97 (m, 3H), 6.56-6.61 (m, 0.5H), 6.25-6.27 (m, 0.5H), 6.07-6.11 $(\mathrm{m}, 0.5 \mathrm{H}), 5.55-5.62(\mathrm{~m}, 2 \mathrm{H}), 3.79-3.91(\mathrm{~m}, 2 \mathrm{H}), 2.98(\mathrm{~d}, J=16.0 \mathrm{~Hz}, 0.5 \mathrm{H}), 2.92(\mathrm{~d}, J=16.0 \mathrm{~Hz}$, $0.5 \mathrm{H}), 2.86(\mathrm{~d}, J=16.0 \mathrm{~Hz}, 0.5 \mathrm{H}), 2.72(\mathrm{~d}, J=16.0 \mathrm{~Hz}, 0.5 \mathrm{H}) ;{ }^{13} \mathrm{C} \mathrm{NMR}\left(100 \mathrm{MHz}, \mathrm{CDCl}_{3}\right.$, two rotamers) $\delta 166.9,166.7,166.6,166.4,161.4(\mathrm{~d}, J=252.6 \mathrm{~Hz}), 161.2(\mathrm{~d}, J=253.2 \mathrm{~Hz}), 143.3(\mathrm{~d}, \mathrm{~J}$ $=9.0 \mathrm{~Hz}), 140.1(\mathrm{~d}, J=7.0 \mathrm{~Hz}), 139.7,139.6,136.3,136.2,136.1,136.0,132.5(\mathrm{~d}, J=8.8 \mathrm{~Hz})$, 131.8 (d, $J=8.6 \mathrm{~Hz}), 131.7,131.0,130.1$ (d, $J=20.2 \mathrm{~Hz}), 129.5,129.4,129.3,129.1,128.6,128.5$, 128.4, 127.7, 127.6, 126.9 (d, $J=2.5 \mathrm{~Hz}), 126.7$ (d, $J=2.7 \mathrm{~Hz}), 116.4$ (d, $J=21.8 \mathrm{~Hz}), 116.0$ (d, $J$ $=21.9 \mathrm{~Hz}), 100.3,100.2,100.0,99.9,99.8,51.9,51.8,51.7,51.6,43.0,42.3$; ESI-MS $\mathrm{m} / z 705.0(\mathrm{M}$ $+\mathrm{H})^{+}$; HRMS calcd for $\mathrm{C}_{29} \mathrm{H}_{24} \mathrm{FI}_{2} \mathrm{~N}_{2} \mathrm{O}_{2}{ }^{+}(\mathrm{M}+\mathrm{H})^{+} 704.9906$, found 704.9907 .

Methyl 4-(N-benzyl-3-(benzyl(2-iodophenyl)amino)-3-oxopropanamido)-3-iodobenzoate (3g)<smiles>CC(=O)c1ccc(N(Cc2ccccc2)C(=O)CC(=O)N(Cc2ccccc2)c2ccccc2I)c(I)c1</smiles>

Light yellow solid, m.p. $162-163{ }^{\circ} \mathrm{C} .{ }^{1} \mathrm{H}$ NMR $\left(400 \mathrm{MHz}, \mathrm{CDCl}_{3}\right) \delta 8.49(2 \mathrm{~d}, \mathrm{~J}=2.0 \mathrm{~Hz}, 1 \mathrm{H}), 7.81-$ $7.85(\mathrm{~m}, 1.5 \mathrm{H}), 7.56(\mathrm{~d}, \mathrm{~J}=2.0 \mathrm{~Hz}, 0.5 \mathrm{H}), 7.17-7.28(\mathrm{~m}, 11 \mathrm{H}), 6.92-7.03(\mathrm{~m}, 2 \mathrm{H}), 6.18(2 \mathrm{~d}, \mathrm{~J}=8.0$ $\mathrm{Hz}, 1 \mathrm{H}), 5.55-5.62(\mathrm{~m}, 2 \mathrm{H}), 3.82-3.97(\mathrm{~m}, 5 \mathrm{H}), 2.99(\mathrm{~d}, J=16.0 \mathrm{~Hz}, 0.5 \mathrm{H}), 2.92(\mathrm{~d}, J=16.0 \mathrm{~Hz}$, $0.5 \mathrm{H}), 2.84(\mathrm{~d}, J=16.0 \mathrm{~Hz}, 0.5 \mathrm{H}), 2.71(\mathrm{~d}, J=16.0 \mathrm{~Hz}, 0.5 \mathrm{H}) ;{ }^{13} \mathrm{C} \mathrm{NMR}\left(100 \mathrm{MHz}, \mathrm{CDCl}_{3}\right) \delta$ $166.5,166.3,166.2,166.0,164.8,164.7,147.2,147.1,143.2,141.2,141.1,140.1,140.0,136.3$, $136.2,135.9,135.8,131.7,131.6,131.4,131.0,130.9,130.5,130.2,130.1,130.0,129.5,129.4$, $129.2,129.2,129.1,128.6,128.5,128.4,128.3,127.8,127.6,100.3,100.1,99.9,52.7,51.8,51.7$, 51.6, 51.5, 43.1, 42.4; ESI-MS $m / z$. $745.0(\mathrm{M}+\mathrm{H})^{+}$; HRMS calcd for $\mathrm{C}_{31} \mathrm{H}_{26} \mathrm{I}_{2} \mathrm{~N}_{2} \mathrm{NaO}_{4}{ }^{+}(\mathrm{M}+\mathrm{Na})^{+}$ 766.9874 , found 766.9868 .

$N^{1}$-(4-acetyl-2-iodophenyl)- $N^{1}, N^{3}$-dibenzyl- $N^{3}$-(2-iodophenyl)malonamide (3h)<smiles>CC(=O)c1ccc(N(Br)C(=O)CC(=O)N(Cc2ccccc2)c2ccccc2I)c(I)c1</smiles>

White solid, m.p. $149-150{ }^{\circ} \mathrm{C} .{ }^{1} \mathrm{H}$ NMR $\left(400 \mathrm{MHz}, \mathrm{CDCl}_{3}\right) \delta 8.38(\mathrm{~s}, 1 \mathrm{H}), 7.85 \& 7.81(2 \mathrm{~d}, \mathrm{~J}=8.0$ $\mathrm{Hz}, 1 \mathrm{H}), 7.72 \& 7.36(2 \mathrm{~d}, J=8.0 \mathrm{~Hz}, 1 \mathrm{H}), 7.07-7.24(\mathrm{~m}, 10.5 \mathrm{H}), 7.06-7.08(\mathrm{~m}, 0.5 \mathrm{H}), 6.91-7.00(\mathrm{~m}$, $2 \mathrm{H}), 6.25(\mathrm{~d}, J=8.0 \mathrm{~Hz}, 0.5 \mathrm{H}), 6.05(\mathrm{~d}, J=8.0 \mathrm{~Hz}, 0.5 \mathrm{H}), 5.57-5.63(\mathrm{~m}, 2 \mathrm{H}), 3.80-3.99(\mathrm{~m}, 2 \mathrm{H})$, $2.98(\mathrm{~d}, J=16.0 \mathrm{~Hz}, 0.5 \mathrm{H}), 2.92(\mathrm{~d}, J=16.0 \mathrm{~Hz}, 0.5 \mathrm{H}), 2.83(\mathrm{~d}, J=16.0 \mathrm{~Hz}, 0.5 \mathrm{H}), 2.68(\mathrm{~d}, J=$ $16.0 \mathrm{~Hz}, 0.5 \mathrm{H}$ ), 2.55\&2.52 (two s, 3H); ${ }^{13} \mathrm{C} \mathrm{NMR}\left(100 \mathrm{MHz}, \mathrm{CDCl}_{3}\right) \delta 195.8,195.6,166.5,166.3$, $166.2,165.9,147.2,147.1,143.2,143.1,140.2,140.1,139.9,139.8,136.3,136.2,135.9,135.8$, $131.9,131.6,131.2,130.8,130.3,130.1,129.6,129.5,129.4,129.3,129.2,129.1,128.9,128.6$, 128.5, 128.4, 127.8, 127.7, 127.6, 100.9, 100.6, 100.3, 99.9, 51.8, 51.7, 51.5, 43.1, 42.3, 26.9, 26.8; ESI-MS $m / z$ 729.0 $(\mathrm{M}+\mathrm{H})^{+} ;$HRMS calcd for $\mathrm{C}_{31} \mathrm{H}_{27} \mathrm{I}_{2} \mathrm{~N}_{2} \mathrm{O}_{3}{ }^{+}(\mathrm{M}+\mathrm{H})^{+}$729.0106, found 729.0107.

$N^{I}$-(4-acetyl-2-iodophenyl)- $N^{I}, N^{3}$-dibenzyl- $N^{3}$-(2-iodo-4-methylphenyl)malonamide (3i) 


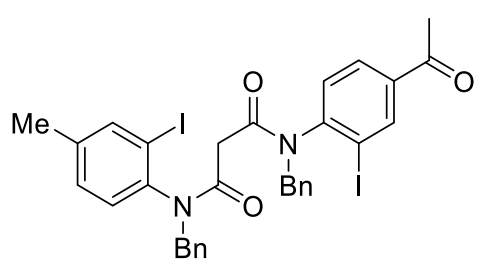

White solid, m.p. $156-157^{\circ} \mathrm{C} .{ }^{1} \mathrm{H}$ NMR (400 MHz, $\left.\mathrm{CDCl}_{3}\right) \delta 8.40 \& 8.39(2 \mathrm{~s}, 1 \mathrm{H}), 7.73$ (dd, $J=8.0$ $\mathrm{Hz}, 2.0 \mathrm{~Hz}, 0.5 \mathrm{H}), 7.67 \& 7.63(2 \mathrm{~d}, J=1.2 \mathrm{~Hz}, 1 \mathrm{H}), 7.37(\mathrm{dd}, J=8.4 \mathrm{~Hz}, 2.0 \mathrm{~Hz}, 0.5 \mathrm{H}), 7.17-7.26$ $(\mathrm{m}, 10 \mathrm{H}), 7.08 \& 6.78(2 \mathrm{~d}, J=8.0 \mathrm{~Hz}, 1 \mathrm{H}$, rotamers), $6.97 \& 6.66(2 \mathrm{dd}, J=8.0 \mathrm{~Hz}, 2.0 \mathrm{~Hz}, 1 \mathrm{H})$, $6.27 \& 5.84(2 \mathrm{~d}, J=8.0 \mathrm{~Hz}, 1 \mathrm{H}), 5.54-5.66(\mathrm{~m}, 2 \mathrm{H}), 3.95(\mathrm{~d}, J=14.8 \mathrm{~Hz}, 0.5 \mathrm{H}), 3.88 \& 3.85(2 \mathrm{~s}, 1 \mathrm{H})$, $3.76(\mathrm{~d}, J=14.4 \mathrm{~Hz}, 0.5 \mathrm{H}), 2.98(\mathrm{~d}, J=16.0 \mathrm{~Hz}, 0.5 \mathrm{H}), 2.93(\mathrm{~d}, J=15.6 \mathrm{~Hz}, 0.5 \mathrm{H}), 2.81$ (d, $J=$ $15.6 \mathrm{~Hz}, 0.5 \mathrm{H}), 2.70(\mathrm{~d}, J=16.0 \mathrm{~Hz}, 0.5 \mathrm{H}), 2.55 \& 2.51(2 \mathrm{~s}, 3 \mathrm{H}), 2.26 \& 2.24(2 \mathrm{~s}, 3 \mathrm{H}) ;{ }^{13} \mathrm{C} \mathrm{NMR}$ $\left(100 \mathrm{MHz}, \mathrm{CDCl}_{3}\right) \delta 195.6,195.4,166.6,166.4,166.3,166.0,147.4,147.3,140.7,140.6,140.5$, $140.4,139.9,139.8,138.1,137.9,136.5,136.4,136.1,136.0,132.1,131.4,131.0,130.3,130.2$, $129.8,129.6,129.5,129.3,129.2,128.7,128.5,128.4,128.3,127.7,127.5,100.9,100.6,99.9,99.5$, 51.9, 51.8, 51.7, 51.5, 43.1, 42.2, 26.6, 20.5, 20.4; ESI-MS m/z $743.0(\mathrm{M}+\mathrm{H})^{+}$; HRMS calcd for $\mathrm{C}_{32} \mathrm{H}_{29} \mathrm{I}_{2} \mathrm{~N}_{2} \mathrm{O}_{3}{ }^{+}(\mathrm{M}+\mathrm{H})^{+}$743.0262, found 743.0259.

\section{General Procedure for the Formation of Spirocyclic Bis-oxindoles:}

An example for the general procedure of spirocyclic bis-oxindoles: The reaction mixture of diamide substrate 1a (534 mg, $1.0 \mathrm{mmol})$, CuI (38 mg, $0.2 \mathrm{mmol}), \mathrm{L} 4$ (96 mg, $0.4 \mathrm{mmol})$ and $\mathrm{Cs}_{2} \mathrm{CO}_{3}(978 \mathrm{mg}, 3.0 \mathrm{mmol})$ in 1,4-dioxane $(10 \mathrm{~mL})$ were stirred at appointed temperature for 24 hours. Then $\mathrm{H}_{2} \mathrm{O}(25.0 \mathrm{~mL})$ and ethyl acetate $(25.0 \mathrm{~mL})$ were added into the mixture. The organic phase was separated and the aqueous phase was extracted with ethyl acetate $(25.0 \mathrm{~mL} \times 3)$. The combined organic phase was washed with $\mathrm{H}_{2} \mathrm{O}$ and brine, dried over $\mathrm{Na}_{2} \mathrm{SO}_{4}$. The solvent was removed under reduced pressure. The residue was purified by flash chromatography (ethyl acetate/petroleum ether $=1 / 10$ to $1 / 3)$ to afford the desired product $\mathbf{2 a}(241 \mathrm{mg}, 87 \%$ yield, $91 \%$ ee, white solid). Other spirocyclic bis-oxindole products were synthesized according to similar procedure in $0.2 \mathrm{mmol}$ scale.

\section{(S)-1,1'-dimethyl-3,3'-spirobi[indoline]-2,2'-dione (2a)}<smiles>CN(C(=O)C1(c2ccccc2)C(=O)N(C)c2ccccc21)c1ccccc1</smiles>

$47.8 \mathrm{mg}, 86 \%$ yield $\left(0.2 \mathrm{mmol}\right.$ scale), white solid, m.p. $215-216^{\circ} \mathrm{C} .{ }^{1} \mathrm{H}$ NMR $\left(400 \mathrm{MHz}, \mathrm{CDCl}_{3}\right) \delta$ 7.34-7.40 (m, 2H), 7.00-7.04 (m, 2H), 6.97 (d, $J=8.0 \mathrm{~Hz}, 2 \mathrm{H}), 6.90$ (dd, $J=7.6 \mathrm{~Hz}, 0.8 \mathrm{~Hz}, 2 \mathrm{H})$, $3.31(\mathrm{~s}, 6 \mathrm{H}) ;{ }^{13} \mathrm{C} \mathrm{NMR}\left(100 \mathrm{MHz}, \mathrm{CDCl}_{3}\right) \delta 172.2,145.3,129.6,127.8,123.9,123.4,108.9,62.3$, 33.9; ESI-MS $m / z 279.1\left(\mathrm{M}+\mathrm{H}^{+}\right)$, HRMS calcd for $\mathrm{C}_{17} \mathrm{H}_{15} \mathrm{~N}_{2} \mathrm{O}_{2}{ }^{+}(\mathrm{M}+\mathrm{H})^{+} 279.1128$, found 279.1129; HPLC Chiralcel OD-H (hexane $/ \mathrm{i}-\mathrm{PrOH}=70: 30,1.0 \mathrm{ml} / \mathrm{min}$ ) $\tau_{\text {major }}=12.0 \mathrm{~min}, \tau_{\text {minor }}=$ $29.2 \mathrm{~min} ;[\alpha]_{\mathrm{D}}^{25}-41.2\left(\right.$ c $1.0, \mathrm{CHCl}_{3}, 91 \%$ ee $)$.

(S)-1,1'-diethyl-3,3'-spirobi[indoline]-2,2'-dione (2b) 
<smiles>CCN1C(=O)C2(C(=O)N(C)c3ccccc32)c2ccccc21</smiles>

$55.1 \mathrm{mg}, 90 \%$ yield, light yellow solid, m.p. $222-223{ }^{\circ} \mathrm{C} .{ }^{1} \mathrm{H}$ NMR $\left(400 \mathrm{MHz}, \mathrm{CDCl}_{3}\right) \delta$ 7.32-7.36 (m, 2H), 6.97-7.02 (m, 4H), $6.87(\mathrm{~d}, J=7.2 \mathrm{~Hz}, 2 \mathrm{H}), 3.80-3.87(\mathrm{~m}, 2 \mathrm{H}), 1.34(\mathrm{t}, J=7.2 \mathrm{~Hz}, 3 \mathrm{H})$; ${ }^{13} \mathrm{C}$ NMR $\left(100 \mathrm{MHz}, \mathrm{CDCl}_{3}\right) \delta 171.8,144.4,129.5,128.2,123.9,123.1,109.0,62.3,35.2,12.8$; ESI-MS $m / z$ 307.1 $\left(\mathrm{M}+\mathrm{H}^{+}\right)$, HRMS calcd for $\mathrm{C}_{19} \mathrm{H}_{19} \mathrm{~N}_{2} \mathrm{O}_{2}{ }^{+}(\mathrm{M}+\mathrm{H})^{+}$307.1441, found 307.1442; HPLC Chiralcel OD-H (hexane $/ \mathrm{i}-\mathrm{PrOH}=70: 30,1.0 \mathrm{ml} / \mathrm{min}$ ) $\tau_{\text {major }}=7.5 \mathrm{~min}, \tau_{\text {minor }}=10.8 \mathrm{~min}$; $[\alpha]_{\mathrm{D}}^{25}-57.4\left(c\right.$ 1.0, $\mathrm{CHCl}_{3}, 94 \%$ ee $)$.

(S)-1,1'-diallyl-3,3'-spirobi[indoline]-2,2'-dione (2c)<smiles>C=CCN1C(=O)C(C2c3ccccc3C(=O)N2CC=C)c2ccccc21</smiles>

$57.4 \mathrm{mg}, 87 \%$ yield, yellow solid, m.p. $170-171{ }^{\circ} \mathrm{C} .{ }^{1} \mathrm{H}$ NMR $\left(400 \mathrm{MHz}, \mathrm{CDCl}_{3}\right) \delta$ 7.30-7.36 (m, 2H), 7.02-7.04 (m, 2H), 6.88-6.95 (m, 4H), 5.86-5.93 (m, 2H), 5.35 (d, J=17.2 Hz, 2H), 5.27 (d, $J$ $=10.8 \mathrm{~Hz}, 2 \mathrm{H}), 4.08-4.28(\mathrm{~m}, 4 \mathrm{H}) ;{ }^{13} \mathrm{C} \mathrm{NMR}\left(100 \mathrm{MHz}, \mathrm{CDCl}_{3}\right) \delta 172.0,144.5,130.6,129.5,127.8$, 123.9, 123.3, 117.6, 109.7, 62.4, 42.8; ESI-MS $m / z 331.1\left(\mathrm{M}+\mathrm{H}^{+}\right)$, HRMS calcd for $\mathrm{C}_{21} \mathrm{H}_{19} \mathrm{~N}_{2} \mathrm{O}_{2}{ }^{+}$ $(\mathrm{M}+\mathrm{H})^{+} 331.1441$, found 331.1441; HPLC Chiralcel OD-H (hexane/i-PrOH $=70: 30,1.0 \mathrm{ml} / \mathrm{min}$ ) $\tau_{\text {major }}=7.7 \mathrm{~min}, \tau_{\text {minor }}=9.4 \mathrm{~min} ;[\alpha]_{\mathrm{D}}{ }^{25}-74.2\left(c 1.0, \mathrm{CHCl}_{3}, 93 \%\right.$ ee $)$.

(S)-1,1'-dibenzyl-3,3'-spirobi[indoline]-2,2'-dione (2d)<smiles>O=C1N(Cc2ccccc2)c2ccccc2C12C(=O)N(Cc1ccccc1)c1ccccc12</smiles>

$81.7 \mathrm{mg}$, 95\% yield, yellow solid, m.p. $110-111{ }^{\circ} \mathrm{C} .{ }^{1} \mathrm{H}$ NMR (400 MHz, $\left.\mathrm{CDCl}_{3}\right) \delta$ 7.30-7.36 (m, 2H), 7.02-7.04 (m, 2H),6.88-6.95 (m, 4H), 5.86-5.93 (m, 2H), $5.35(\mathrm{~d}, J=17.2 \mathrm{~Hz}, 2 \mathrm{H}), 5.27$ (d, $J$ $=10.8 \mathrm{~Hz}, 2 \mathrm{H}), 4.08-4.28(\mathrm{~m}, 4 \mathrm{H}) ;{ }^{13} \mathrm{C} \mathrm{NMR}\left(100 \mathrm{MHz}, \mathrm{CDCl}_{3}\right) \delta 172.0,144.5,130.6,129.5,127.8$, 123.9, 123.3, 117.6, 109.7, 62.4, 42.8; ESI-MS $m / z$ 431.1 (M+ $\left.\mathrm{H}^{+}\right)$, HRMS calcd for $\mathrm{C}_{29} \mathrm{H}_{23} \mathrm{~N}_{2} \mathrm{O}_{2}{ }^{+}$ $(\mathrm{M}+\mathrm{H})^{+}$431.1754, found 431.1753; HPLC Chiralpak AS-H (hexane/i-PrOH $=80: 20,1.0 \mathrm{ml} / \mathrm{min}$ ) $\tau_{\text {major }}=25.8 \mathrm{~min}, \tau_{\text {minor }}=17.8 \mathrm{~min} ;[\alpha]_{\mathrm{D}}{ }^{25}-67.0\left(c 1.0, \mathrm{CHCl}_{3}, 96 \% e e\right)$.

(S)-1,1'-dibenzyl-5,5'-dimethyl-3,3'-spirobi[indoline]-2,2'-dione (2e) 


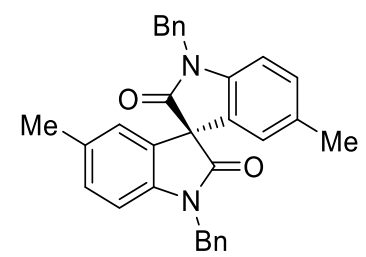

$88.9 \mathrm{mg}$, 97\% yield, yellow solid, m.p. 103-104 ${ }^{\circ} \mathrm{C} .{ }^{1} \mathrm{H}$ NMR (400 MHz, $\left.\mathrm{CDCl}_{3}\right) \delta$ 7.36-7.43 (m, $8 \mathrm{H}), 7.28-7.31(\mathrm{~m}, 2 \mathrm{H}), 7.05(\mathrm{~d}, J=8.0 \mathrm{~Hz}, 2 \mathrm{H}), 6.77(\mathrm{~s}, 2 \mathrm{H}), 6.72(\mathrm{~d}, J=8.0 \mathrm{~Hz}, 2 \mathrm{H}), 5.08(\mathrm{~d}, J=$ $15.6 \mathrm{~Hz}, 2 \mathrm{H}), 4.99$ (d, $J=15.6 \mathrm{~Hz}, 2 \mathrm{H}), 2.24(\mathrm{~s}, 6 \mathrm{H}) ;{ }^{13} \mathrm{C} \mathrm{NMR}\left(100 \mathrm{MHz}, \mathrm{CDCl}_{3}\right) \delta 172.6,142.0$, 135.4, 133.1, 129.9, 128.9, 128.1, 127.7, 127.2, 124.7, 109.8, 62.6, 44.3, 21.1; ESI-MS $m / z 459.1$ $\left(\mathrm{M}+\mathrm{H}^{+}\right)$, HRMS calcd for $\mathrm{C}_{31} \mathrm{H}_{27} \mathrm{~N}_{2} \mathrm{O}_{2}{ }^{+}(\mathrm{M}+\mathrm{H})^{+}$459.2067, found 459.2061; HPLC Chiralcel OD$\mathrm{H}$ (hexane $/ \mathrm{i}-\mathrm{PrOH}=85: 15,1.0 \mathrm{ml} / \mathrm{min}) \tau_{\text {major }}=14.2 \mathrm{~min}, \tau_{\text {minor }}=20.0 \mathrm{~min} ;[\alpha]_{\mathrm{D}}{ }^{25}-76.4(c 1.0$, $\mathrm{CHCl}_{3}, 95 \%$ ee).

\section{(S)-1,1'-dibenzyl-4,4'-dimethyl-3,3'-spirobi[indoline]-2,2'-dione (2f)}

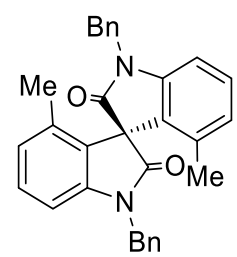

$44 \mathrm{mg}, 48 \%$ yield, yellow solid, m.p. 89-90 ${ }^{\circ} \mathrm{C} .{ }^{1} \mathrm{H}$ NMR $\left(400 \mathrm{MHz}, \mathrm{CDCl}_{3}\right) \delta$ 7.27-7.44 (m, 10H), 7.14-7.18 (m, 2H), 6.78 (d, $J=8.0 \mathrm{~Hz}, 2 \mathrm{H}), 6.72(\mathrm{~d}, J=8.0 \mathrm{~Hz}, 2 \mathrm{H}), 5.08$ (d, $J=15.6 \mathrm{~Hz}, 2 \mathrm{H}), 4.99$ $(\mathrm{d}, J=15.6 \mathrm{~Hz}, 2 \mathrm{H}), 1.66(\mathrm{~s}, 6 \mathrm{H}) ;{ }^{13} \mathrm{C} \mathrm{NMR}\left(100 \mathrm{MHz}, \mathrm{CDCl}_{3}\right) \delta 171.7,144.3,135.4,134.9,129.3$, 128.8, 127.8, 127.5, 125.1, 124.3, 62.1, 44.6, 17.3; ESI-MS $m / z$ 459.1 $\left(\mathrm{M}+\mathrm{H}^{+}\right)$, HRMS calcd for $\mathrm{C}_{31} \mathrm{H}_{27} \mathrm{~N}_{2} \mathrm{O}_{2}{ }^{+}(\mathrm{M}+\mathrm{H})^{+}$459.2067, found 459.2061; HPLC Chiralcel OD-H (hexane/i-PrOH = 85:15, $1.0 \mathrm{ml} / \mathrm{min}) \tau_{\text {major }}=20.3 \mathrm{~min}, \tau_{\text {minor }}=18.5 \mathrm{~min} ;[\alpha]_{\mathrm{D}}^{25}-8.0\left(c 0.7, \mathrm{CHCl}_{3}, 91 \% e e\right)$.

(S)-1,1'-dibenzyl-5,5'-dimethoxy-3,3'-spirobi[indoline]-2,2'-dione (2g)<smiles>COc1cccc(C23C(=O)C(c4cc(OC)ccc42)C(Cc2ccccc2)N(Cc2ccccc2)C3=O)c1</smiles>

95.1 mg, 97\% yield, yellow solid, m.p. 116-117 ${ }^{\circ} \mathrm{C} .{ }^{1} \mathrm{H}$ NMR $\left(400 \mathrm{MHz}, \mathrm{CDCl}_{3}\right) \delta$ 7.27-7.41 (m, $10 \mathrm{H}), 6.77(\mathrm{dd}, \mathrm{J}=8.4 \mathrm{~Hz}, 2.4 \mathrm{~Hz}, 2 \mathrm{H}), 6.71(\mathrm{~d}, J=8.4 \mathrm{~Hz}, 2 \mathrm{H}), 6.52(\mathrm{~d}, J=2.4 \mathrm{~Hz}, 2 \mathrm{H}), 5.05(\mathrm{~d}$, $J=15.6 \mathrm{~Hz}, 2 \mathrm{H}), 4.96(\mathrm{~d}, J=15.6 \mathrm{~Hz}, 2 \mathrm{H}), 3.67(\mathrm{~s}, 6 \mathrm{H}) ;{ }^{13} \mathrm{C} \mathrm{NMR}\left(100 \mathrm{MHz}, \mathrm{CDCl}_{3}\right) \delta 172.2$, 156.4, 137.6, 135.3, 128.9, 127.7, 127.1, 114.3, 110.9, 110.5, 63.1, 55.8, 44.4; ESI-MS $m / z$ 491.1 $\left(\mathrm{M}+\mathrm{H}^{+}\right)$, HRMS calcd for $\mathrm{C}_{31} \mathrm{H}_{27} \mathrm{~N}_{2} \mathrm{O}_{4}{ }^{+}(\mathrm{M}+\mathrm{H})^{+}$491.1965, found 491.1961; HPLC Chiralpak AS$\mathrm{H}$ (hexane $/ \mathrm{i}-\mathrm{PrOH}=80: 20,1.0 \mathrm{ml} / \mathrm{min}) \tau_{\text {major }}=35.0 \mathrm{~min}, \tau_{\text {minor }}=45.0 \mathrm{~min} ;[\alpha]_{\mathrm{D}}{ }^{25}-47.6(c 1.0$, $\mathrm{CHCl}_{3},>99 \%$ ee).

(S)-1,1'-dibenzyl-5,5'-dibromo-3,3'-spirobi[indoline]-2,2'-dione (2h) 
<smiles>O=C(Nc1ccccc1)C1(Cc2ccccc2)C(=O)N(Cc2ccccc2)c2ccc(Br)cc21</smiles>

$99.6 \mathrm{mg}, 85 \%$ yield, yellow solid, m.p. $211-212{ }^{\circ} \mathrm{C} .{ }^{1} \mathrm{H}$ NMR $\left(400 \mathrm{MHz}, \mathrm{CDCl}_{3}\right) \delta$ 7.29-7.40 (m, $12 \mathrm{H}), 7.05(\mathrm{~d}, J=1.6 \mathrm{~Hz}, 2 \mathrm{H}), 6.70(\mathrm{~d}, J=8.4 \mathrm{~Hz}, 2 \mathrm{H}), 5.08(\mathrm{~d}, J=16.0 \mathrm{~Hz}, 2 \mathrm{H}), 4.93(\mathrm{~d}, J=16.0$ $\mathrm{Hz}, 2 \mathrm{H}) ;{ }^{13} \mathrm{C}$ NMR $\left(100 \mathrm{MHz}, \mathrm{CDCl}_{3}\right) \delta 171.3,143.4,134.5,132.8,129.1,128.9,128.1,127.2$, 127.1, 116.2, 111.6, 62.0, 44.5; ESI-MS $m / z$ 587.0 $\left(\mathrm{M}+\mathrm{H}^{+}\right)$, HRMS calcd for $\mathrm{C}_{29} \mathrm{H}_{21} \mathrm{Br}_{2} \mathrm{~N}_{2} \mathrm{O}_{2}{ }^{+}(\mathrm{M}$ $+\mathrm{H})^{+} 586.9965$, found 586.9967 ; HPLC Chiralpak AS-H (hexane $/ \mathrm{i}-\mathrm{PrOH}=80: 20,1.0 \mathrm{ml} / \mathrm{min}$ ) $\tau_{\text {major }}$ $=14.9 \mathrm{~min}, \tau_{\mathrm{minor}}=17.5 \mathrm{~min} ;[\alpha]_{\mathrm{D}}{ }^{25}-62.4\left(c 1.0, \mathrm{CHCl}_{3}, 93 \%\right.$ ee $)$.

(S)-1,1'-dibenzyl-5,5'-dichloro-3,3'-spirobi[indoline]-2,2'-dione (2i)<smiles>O=C(c1ccccc1)[C@@]1(Cc2ccccc2)C(=O)N(Cc2ccccc2)c2ccc(Cl)cc21</smiles>

$96.6 \mathrm{mg}, 97 \%$ yield, yellow solid, m.p. $159-160{ }^{\circ} \mathrm{C} .{ }^{1} \mathrm{H}$ NMR $\left(400 \mathrm{MHz}, \mathrm{CDCl}_{3}\right) \delta$ 7.28-7.39 (m, $10 \mathrm{H}), 7.24(\mathrm{dd}, J=8.4 \mathrm{~Hz}, 2.0 \mathrm{~Hz}, 2 \mathrm{H}), 6.92(\mathrm{~d}, J=2.0 \mathrm{~Hz}, 2 \mathrm{H}), 6.75(\mathrm{~d}, J=8.4 \mathrm{~Hz}, 2 \mathrm{H}), 5.08(\mathrm{~d}$, $J=16.0 \mathrm{~Hz}, 2 \mathrm{H}), 4.95(\mathrm{~d}, J=16.0 \mathrm{~Hz}, 2 \mathrm{H}) ;{ }^{13} \mathrm{C} \mathrm{NMR}\left(100 \mathrm{MHz}, \mathrm{CDCl}_{3}\right) \delta 171.4,142.9,134.5$, 129.9, 129.1, 129.0, 128.6, 128.0, 127.1, 124.5, 111.2, 62.2, 44.6; ESI-MS $m / z 499.0\left(\mathrm{M}+\mathrm{H}^{+}\right)$, HRMS calcd for $\mathrm{C}_{29} \mathrm{H}_{21} \mathrm{Cl}_{2} \mathrm{~N}_{2} \mathrm{O}_{2}{ }^{+}(\mathrm{M}+\mathrm{H})^{+}$499.0975, found 499.0979; HPLC Chiralcel OJ-H (hexane $/ \mathrm{i}-\mathrm{PrOH}=80: 20,1.0 \mathrm{ml} / \mathrm{min}) \tau_{\text {major }}=16.2 \mathrm{~min}, \tau_{\text {minor }}=23.7 \mathrm{~min} ;[\alpha]_{\mathrm{D}}{ }^{25}-107.4\left(c 1.0, \mathrm{CHCl}_{3}\right.$, $94 \% e e$ ).

\section{Dimethyl (S)-1,1'-dibenzyl-2,2'-dioxo-3,3'-spirobi[indoline]-5,5'-dicarboxylate (2j)}<smiles>CC(=O)c1ccc2c(c1)C(C(=O)n1c(=O)n(Cc3ccccc3)c3ccc(C(C)=O)cc31)C(=O)N2Cc1ccccc1</smiles>

$108 \mathrm{mg}$, 99\% yield, yellow solid, m.p. 259-260 ${ }^{\circ} \mathrm{C} .{ }^{1} \mathrm{H}$ NMR (400 MHz, $\mathrm{CDCl}_{3}$ ) $\delta 8.01$ (dd, $J=8.4$ $\mathrm{Hz}, 1.6 \mathrm{~Hz}, 2 \mathrm{H}), 7.60(\mathrm{~d}, J=1.6 \mathrm{~Hz}, 2 \mathrm{H}), 7.26-7.39(\mathrm{~m}, 10 \mathrm{H}), 6.89(\mathrm{~d}, J=8.4 \mathrm{~Hz}, 2 \mathrm{H}), 5.16(\mathrm{~d}, J$ $=16.0 \mathrm{~Hz}, 2 \mathrm{H}), 4.96(\mathrm{~d}, J=16.0 \mathrm{~Hz}, 2 \mathrm{H}), 3.81(\mathrm{~s}, 6 \mathrm{H}) ;{ }^{13} \mathrm{C} \mathrm{NMR}\left(100 \mathrm{MHz}, \mathrm{CDCl}_{3}\right) \delta 172.1,166.2$, 148.5, 134.4, 132.4, 129.1, 128.1, 127.2, 127.0, 125.6, 125.4, 109.8, 61.8, 52.2, 44.6; ESI-MS $\mathrm{m} / \mathrm{z}$ $547.1\left(\mathrm{M}+\mathrm{H}^{+}\right)$, HRMS calcd for $\mathrm{C}_{33} \mathrm{H}_{27} \mathrm{~N}_{2} \mathrm{O}_{6}{ }^{+}(\mathrm{M}+\mathrm{H})^{+}$547.1864, found 547.1868; HPLC Chiralcel OD-H (hexane $/ \mathrm{i}-\mathrm{PrOH}=60: 40,1.0 \mathrm{ml} / \mathrm{min}) \tau_{\text {major }}=18.2 \mathrm{~min}, \tau_{\text {minor }}=26.9 \mathrm{~min} ;[\alpha]_{\mathrm{D}}{ }^{25}-$ 112.0 (c 1.0, $\mathrm{CHCl}_{3}, \quad>99 \%$ ee).

(S)-5,5'-diacetyl-1,1'-dibenzyl-3,3'-spirobi[indoline]-2,2'-dione (2k) 


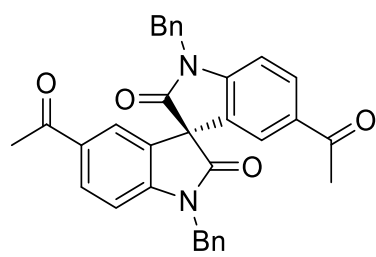

$92.7 \mathrm{mg}, 90 \%$ yield, red solid, m.p. 290-291 ${ }^{\circ} \mathrm{C} .{ }^{1} \mathrm{H}$ NMR (400 MHz, $\left.\mathrm{CDCl}_{3}\right) \delta 7.91$ (d, J=8.0 Hz, 2H), $7.55(\mathrm{~s}, 2 \mathrm{H}), 7.26-7.38(\mathrm{~m}, 10 \mathrm{H}), 6.90(\mathrm{~d}, J=8.0 \mathrm{~Hz}, 2 \mathrm{H}), 5.17$ (d, $J=16.0 \mathrm{~Hz}, 2 \mathrm{H}), 4.95(\mathrm{~d}$, $J=16.0 \mathrm{~Hz}, 2 \mathrm{H}), 2.47$ (s, 6H); ${ }^{13} \mathrm{C}$ NMR $\left(100 \mathrm{MHz}, \mathrm{CDCl}_{3}\right) \delta 196.3,172.1,148.6,134.4,133.0$, 131.7, 129.2, 128.1, 127.6, 127.0, 123.9, 109.7, 61.8, 44.7, 26.4; ESI-MS $m / z 515.1\left(\mathrm{M}+\mathrm{H}^{+}\right)$, HRMS calcd for $\mathrm{C}_{33} \mathrm{H}_{27} \mathrm{~N}_{2} \mathrm{O}_{4}^{+}(\mathrm{M}+\mathrm{H})^{+}$515.1965, found 515.1965; HPLC Chiralcel OJ-H (hexane $/ \mathrm{i}-\mathrm{PrOH}=50: 50,1.0 \mathrm{ml} / \mathrm{min}) \tau_{\text {major }}=26.7 \mathrm{~min}, \tau_{\text {minor }}=50.6 \mathrm{~min} ;[\alpha]_{\mathrm{D}}{ }^{25}-130.8(c 1.0$, $\mathrm{CHCl}_{3},>99 \%$ ee).

\section{(S)-1,1'-dibenzyl-6,6'-dimethyl-3,3'-spirobi[indoline]-2,2'-dione (2I)}<smiles>Cc1cccc(C(=O)C2(C(=O)Nc3ccccc3)C(=O)N(Cc3ccccc3)c3cc(C)ccc32)c1</smiles>

$82.3 \mathrm{mg}, 90 \%$ yield, yellow solid, m.p. $91-92{ }^{\circ} \mathrm{C} .{ }^{1} \mathrm{H}$ NMR $\left(400 \mathrm{MHz}, \mathrm{CDCl}_{3}\right) \delta$ 7.37-7.43 (m, 8H), 7.31-7.33 (m, 2H), 6.85 (d, $J=7.6 \mathrm{~Hz}, 2 \mathrm{H}), 6.83$ (d, $J=7.6 \mathrm{~Hz}, 2 \mathrm{H}), 6.67$ (s, 2H), 5.06 (d, $J=16.0$ $\mathrm{Hz}, 2 \mathrm{H}), 5.01(\mathrm{~d}, J=16.0 \mathrm{~Hz}, 2 \mathrm{H}), 2.33(\mathrm{~s}, 6 \mathrm{H}) ;{ }^{13} \mathrm{C} \mathrm{NMR}\left(100 \mathrm{MHz}, \mathrm{CDCl}_{3}\right) \delta 173.0,139.8,135.4$, 128.9, 127.6, 127.0, 125.1, 124.0, 123.6, 110.7, 62.0, 44.2, 21.9; ESI-MS $m / z 459.1\left(\mathrm{M}+\mathrm{H}^{+}\right)$, HRMS calcd for $\mathrm{C}_{31} \mathrm{H}_{27} \mathrm{~N}_{2} \mathrm{O}_{2}{ }^{+}(\mathrm{M}+\mathrm{H})^{+}$459.2067, found 459.2053; HPLC Chiralcel AS-H (hexane $/ \mathrm{i}-\mathrm{PrOH}=85: 15,1.0 \mathrm{ml} / \mathrm{min}) \tau_{\text {major }}=14.8 \mathrm{~min}, \tau_{\text {minor }}=8.2 \mathrm{~min} ;[\alpha]_{\mathrm{D}}{ }^{25}-45.0\left(c 1.0, \mathrm{CHCl}_{3}\right.$, $99 \%$ ee).

\section{(S)-1,1'-dibenzyl-6,6'-dichloro-3,3'-spirobi[indoline]-2,2'-dione (2m)}<smiles>O=C1Nc2cc(Cl)ccc2C12C(=O)N(Cc1ccccc1)c1cc(Cl)ccc12</smiles>

91.6mg, 92\% yield, yellow solid, m.p. $229-230{ }^{\circ} \mathrm{C} .{ }^{1} \mathrm{H}$ NMR $\left(400 \mathrm{MHz}, \mathrm{CDCl}_{3}\right) \delta$ 7.29-7.38 (m, $10 \mathrm{H}), 7.00$ (dd, $J=8.0 \mathrm{~Hz}, 1.6 \mathrm{~Hz}, 2 \mathrm{H}), 6.81-6.83(\mathrm{~m}, 4 \mathrm{H}), 5.03(\mathrm{~d}, J=16.0 \mathrm{~Hz}, 2 \mathrm{H}), 4.95$ (d, $J=$ $16.0 \mathrm{~Hz}, 2 \mathrm{H}) ;{ }^{13} \mathrm{C} \mathrm{NMR}\left(100 \mathrm{MHz}, \mathrm{CDCl}_{3}\right) \delta 171.9,145.6,135.7,134.5,129.1,128.1,127.0,125.6$, 124.8, 123.6, 110.7, 61.5, 44.5; ESI-MS $m / z$ 499.1 (M + $\left.\mathrm{H}^{+}\right)$, HRMS calcd for $\mathrm{C}_{29} \mathrm{H}_{21} \mathrm{Cl}_{2} \mathrm{~N}_{2} \mathrm{O}_{2}{ }^{+}(\mathrm{M}$ $+\mathrm{H})^{+}$499.0975, found 499.0974; HPLC Chiralcel OD-H (hexane $/ \mathrm{i}-\mathrm{PrOH}=85: 15,1.0 \mathrm{ml} / \mathrm{min}$ ) $\tau_{\text {major }}$ $=11.5 \mathrm{~min}, \tau_{\operatorname{minor}}=15.1 \mathrm{~min} ;[\alpha]_{\mathrm{D}}^{25}-39.1\left(c 1.0, \mathrm{CHCl}_{3}, 96 \%\right.$ ee $)$.

\section{(S)-1-benzyl-1'-methyl-3,3'-spirobi[indoline]-2,2'-dione (4a)}




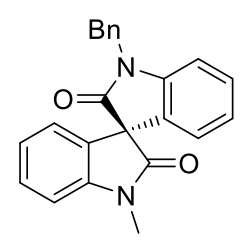

$63.6 \mathrm{mg}, 90 \%$ yield, white solid, m.p. $223-224{ }^{\circ} \mathrm{C} .{ }^{1} \mathrm{H}$ NMR $\left(400 \mathrm{MHz}, \mathrm{CDCl}_{3}\right) \delta$ 7.34-7.40 (m, 5H), 7.28-7.31 (m, 1H), 7.20-7.23 (m, 1H), 7.06-7.08 (m, 1H), 6.97-7.04 (m, 2H), 6.89-6.93 (m, 2H), $6.80(\mathrm{~d}, J=8.0 \mathrm{~Hz}, 1 \mathrm{H}), 5.03(\mathrm{~d}, J=16.0 \mathrm{~Hz}, 1 \mathrm{H}), 4.98(\mathrm{~d}, J=16.0 \mathrm{~Hz}, 1 \mathrm{H}), 3.33(\mathrm{~s}, 6 \mathrm{H}) ;{ }^{13} \mathrm{C} \mathrm{NMR}$ $\left(100 \mathrm{MHz}, \mathrm{CDCl}_{3}\right) \delta 172.5,172.2,145.4,144.3,135.3,129.7,129.5,129.0,127.9,127.8,127.7$, $127.1,124.0,123.9,123.5,123.4,110.0,109.0,62.4,44.3,27.1$; ESI-MS $m / z 355.1\left(\mathrm{M}+\mathrm{H}^{+}\right)$, HRMS calcd for $\mathrm{C}_{23} \mathrm{H}_{19} \mathrm{~N}_{2} \mathrm{O}_{2}{ }^{+}(\mathrm{M}+\mathrm{H})^{+}$355.1441, found 355.1445; HPLC Chiralcel OD-H (hexane $/ \mathrm{i}-\mathrm{PrOH}=85: 15,1.0 \mathrm{ml} / \mathrm{min}) \tau_{\text {major }}=21.7 \mathrm{~min}, \tau_{\text {minor }}=26.2 \mathrm{~min} ;[\alpha]_{\mathrm{D}}{ }^{25}-62.6\left(c 1.0, \mathrm{CHCl}_{3}\right.$, $88 \%$ ee).

\section{(S)-1-allyl-1'-benzyl-3,3'-spirobi[indoline]-2,2'-dione (4b)}<smiles>C=CCN1c2ccccc2C(C(=O)N(Cc2ccccc2)c2ccccc2)c2ccccc21</smiles>

$72.4 \mathrm{mg}$, 95\% yield, yellow solid, m.p. $180-181{ }^{\circ} \mathrm{C} .{ }^{1} \mathrm{H}$ NMR $\left(400 \mathrm{MHz}, \mathrm{CDCl}_{3}\right) \delta$ 7.23-7.40 (m, $7 \mathrm{H}), 6.90-7.04(\mathrm{~m}, 5 \mathrm{H}), 6.80(\mathrm{~d}, J=8.0 \mathrm{~Hz}, 1 \mathrm{H}), 5.88-5.95(\mathrm{~m}, 1 \mathrm{H}), 5.28-5.40(\mathrm{~m}, 2 \mathrm{H}), 5.04(\mathrm{~d}, J$ $=16.0 \mathrm{~Hz}, 1 \mathrm{H}), 4.98(\mathrm{~d}, J=16.0 \mathrm{~Hz}, 1 \mathrm{H}), 4.44-4.46(\mathrm{~m}, 2 \mathrm{H}) ;{ }^{13} \mathrm{C} \mathrm{NMR}\left(100 \mathrm{MHz}, \mathrm{CDCl}_{3}\right) \delta 172.4$, 172.0, 145.5, 145.4, 130.6, 129.6, 129.5, 128.9, 127.7, 127.1, 124.0, 123.9, 123.4, 117.6, 110.0, 109.8, 62.4, 44.3, 42.8; ESI-MS $m / z 381.1\left(\mathrm{M}+\mathrm{H}^{+}\right)$, HRMS calcd for $\mathrm{C}_{25} \mathrm{H}_{21} \mathrm{~N}_{2} \mathrm{O}_{2}{ }^{+}(\mathrm{M}+\mathrm{H})^{+}$ 381.1598 , found 381.1601; HPLC Chiralcel OD-H (hexane $/ \mathrm{i}-\mathrm{PrOH}=95: 5,1.0 \mathrm{ml} / \mathrm{min}$ ) $\tau_{\text {major }}=45.4$ $\min , \tau_{\operatorname{minor}}=55.8 \mathrm{~min} ;[\alpha]_{\mathrm{D}}{ }^{25}-40.2\left(c 1.0, \mathrm{CHCl}_{3}, 92.5 \% e e\right)$.

\section{(S)-1,1'-dibenzyl-5-methyl-3,3'-spirobi[indoline]-2,2'-dione (4c)}

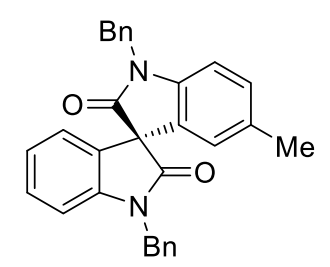

$87.0 \mathrm{mg}, 98 \%$ yield, yellow solid, m.p. $127-128{ }^{\circ} \mathrm{C} .{ }^{1} \mathrm{H}$ NMR $\left(400 \mathrm{MHz}, \mathrm{CDCl}_{3}\right) \delta$ 7.35-7.44 (m, $8 \mathrm{H}), 7.24-7.31(\mathrm{~m}, 3 \mathrm{H}), 7.00-7.06(\mathrm{~m}, 2 \mathrm{H}), 6.93-6.95(\mathrm{~m}, 1 \mathrm{H}), 6.83-6.84(\mathrm{~m}, 1 \mathrm{H}), 6.70-6.76(\mathrm{~m}$, 2H), 4.98-5.15 (m, 4H), 2.23 (s, 3H); ${ }^{13} \mathrm{C}$ NMR (100 MHz, $\left.\mathrm{CDCl}_{3}\right) \delta$ 172.7, 172.4, 144.4, 141.9, 135.3, 135.2, 133.2, 129.9, 129.6, 129.0, 128.9, 128.1, 127.9, 127.8, 127.7, 127.2, 127.1, 124.7, 124.0, 123.5, 110.0, 109.8, 62.5, 44.3, 44.2, 21.1; ESI-MS $m / z$ 445.1 $\left(\mathrm{M}+\mathrm{H}^{+}\right)$, HRMS calcd for $\mathrm{C}_{30} \mathrm{H}_{25} \mathrm{~N}_{2} \mathrm{O}_{2}{ }^{+}(\mathrm{M}+\mathrm{H})^{+}$445.1911, found 445.1913; HPLC Chiralcel OD-H (hexane/i-PrOH = 90:10, $1.0 \mathrm{ml} / \mathrm{min}) \tau_{\text {major }}=25.2 \mathrm{~min}, \tau_{\text {minor }}=33.2 \mathrm{~min} ;[\alpha]_{\mathrm{D}}{ }^{25}-53.4\left(c 1.0, \mathrm{CHCl}_{3}, 93 \%\right.$ ee $)$. 


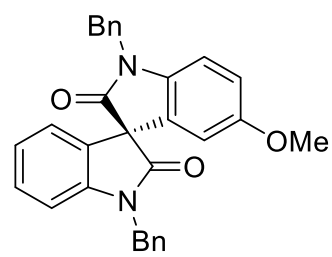

$81.0 \mathrm{mg}, 88 \%$ yield, yellow solid, m.p. $145-146{ }^{\circ} \mathrm{C} .{ }^{1} \mathrm{H}$ NMR $\left(400 \mathrm{MHz}, \mathrm{CDCl}_{3}\right) \delta$ 7.34-7.41 (m, $8 \mathrm{H}), 7.23-7.28(\mathrm{~m}, 3 \mathrm{H}), 7.01-7.03(\mathrm{~m}, 1 \mathrm{H}), 6.93(\mathrm{~d}, J=6.4 \mathrm{~Hz}, 1 \mathrm{H}), 6.82(\mathrm{~d}, J=8.0 \mathrm{~Hz}, 1 \mathrm{H}), 6.76$ $(\mathrm{dd}, J=8.0,2.4 \mathrm{~Hz}, 1 \mathrm{H}), 6.70(\mathrm{~d}, \mathrm{~J}=8.0 \mathrm{~Hz}, 1 \mathrm{H}), 6.50(\mathrm{~d}, J=2.4 \mathrm{~Hz}, 1 \mathrm{H}), 4.95-5.10(\mathrm{~m}, 2 \mathrm{H}), 3.67$ (s, 3H); ${ }^{13} \mathrm{C}$ NMR (100 MHz, $\left.\mathrm{CDCl}_{3}\right) \delta 172.5,172.2,156.4,144.3,137.7,135.3,135.2,129.6,129.0$, $128.9,127.9,127.8,127.7,127.1,127.0,124.0,123.5,114.2,110.9,110.5,110.0,62.9,55.7,44.4$, 44.3; ESI-MS $m / z 461.1\left(\mathrm{M}+\mathrm{H}^{+}\right)$, HRMS calcd for $\mathrm{C}_{30} \mathrm{H}_{25} \mathrm{~N}_{2} \mathrm{O}_{3}{ }^{+}(\mathrm{M}+\mathrm{H})^{+} 461.1860$, found 461.1858; HPLC Chiralcel OJ-H (hexane $/ \mathrm{i}-\mathrm{PrOH}=50: 50,1.0 \mathrm{ml} / \mathrm{min}$ ) $\tau_{\text {major }}=11.5 \mathrm{~min}, \tau_{\text {minor }}=$ $11.6 \mathrm{~min} ;[\alpha]_{\mathrm{D}}{ }^{25}-29.0\left(\right.$ c $1.0, \mathrm{CHCl}_{3}, 97.5 \%$ ee $)$.

\section{(S)-1,1'-dibenzyl-5-chloro-3,3'-spirobi[indoline]-2,2'-dione (4e)}

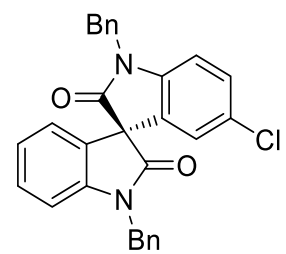

$78.9 \mathrm{mg}, 85 \%$ yield, white solid, m.p. $161-162{ }^{\circ} \mathrm{C} .{ }^{1} \mathrm{H}$ NMR $\left(400 \mathrm{MHz}, \mathrm{CDCl}_{3}\right) \delta$ 7.26-7.42 (m, $11 \mathrm{H}), 7.22(\mathrm{dd}, J=8.4 \mathrm{~Hz}, 2.0 \mathrm{~Hz}, 1 \mathrm{H}), 7.01-7.05(\mathrm{~m}, 1 \mathrm{H}), 6.91-6.94(\mathrm{~m}, 2 \mathrm{H}), 6.84(\mathrm{~d}, J=8.0 \mathrm{~Hz}$, $1 \mathrm{H}), 6.73(\mathrm{~d}, \mathrm{~J}=8.4 \mathrm{~Hz}, 1 \mathrm{H}), 4.96-5.11(\mathrm{~m}, 4 \mathrm{H}) ;{ }^{13} \mathrm{C} \mathrm{NMR}\left(100 \mathrm{MHz}, \mathrm{CDCl}_{3}\right) \delta$ 172.0, 171.9, 144.4, 143.0, 135.0, 134.8, 129.9, 129.1, 129.0, 128.0, 127.9, 127.1, 127.0, 124.5, 124.0, 111.0, 110.2, 62.3, 44.5, 44.4; ESI-MS $m / z 465.1\left(\mathrm{M}+\mathrm{H}^{+}\right)$, HRMS calcd for $\mathrm{C}_{29} \mathrm{H}_{22} \mathrm{ClN}_{2} \mathrm{O}_{2}{ }^{+}(\mathrm{M}+\mathrm{H})^{+} 465.1364$, found 465.1361; HPLC Chiralpak AS-H (hexane $/ \mathrm{i}-\mathrm{PrOH}=80: 20,1.0 \mathrm{ml} / \mathrm{min}$ ) $\tau_{\text {major }}=18.5 \mathrm{~min}, \tau_{\text {minor }}=$ $15.6 \mathrm{~min} ;[\alpha]_{\mathrm{D}}{ }^{25}-50.7\left(c 1.0, \mathrm{CHCl}_{3}, 81 \%\right.$ ee $)$.

(S)-1,1'-dibenzyl-5-fluoro-3,3'-spirobi[indoline]-2,2'-dione (4f)

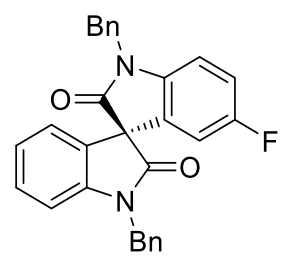

$80.6 \mathrm{mg}$, 90\% yield, yellow solid, m.p. $155-156{ }^{\circ} \mathrm{C} .{ }^{1} \mathrm{H}$ NMR $\left(400 \mathrm{MHz}, \mathrm{CDCl}_{3}\right) \delta$ 7.26-7.40 (m, $11 \mathrm{H}), 7.01-7.05(\mathrm{~m}, 1 \mathrm{H}), 6.93-6.97(\mathrm{~m}, 2 \mathrm{H}), 6.85(\mathrm{~d}, J=8.0 \mathrm{~Hz}, 1 \mathrm{H}), 6.69-6.76(\mathrm{~m}, 2 \mathrm{H}), 4.98-5.10$ $(\mathrm{m}, 4 \mathrm{H}) ;{ }^{13} \mathrm{C}$ NMR $\left(100 \mathrm{MHz}, \mathrm{CDCl}_{3}\right) \delta 172.2,172.0,159.4(\mathrm{~d}, J=220.8 \mathrm{~Hz}), 144.3,140.4,140.3$, $135.0(\mathrm{~d}, J=16.8 \mathrm{~Hz}), 129.9,129.2,129.1,129.0,127.9,127.8,127.1,127.0,123.8$ (d, $J=29.9$ $\mathrm{Hz}), 116.0$ (d, $J=23.3 \mathrm{~Hz}), 112.1$ (d, $J=25.1 \mathrm{~Hz}), 110.6$ (d, $J=8.0 \mathrm{~Hz}), 110.2,62.7,62.6,44.5$, 44.4; ESI-MS $m / z$ 449.1 (M + $\left.\mathrm{H}^{+}\right)$, HRMS calcd for $\mathrm{C}_{29} \mathrm{H}_{22} \mathrm{FN}_{2} \mathrm{O}_{2}{ }^{+}(\mathrm{M}+\mathrm{H})^{+} 449.1660$, found 449.1662; HPLC Chiralpak AS-H (hexane $/ \mathrm{i}-\mathrm{PrOH}=80: 20,1.0 \mathrm{ml} / \mathrm{min}$ ) $\tau_{\text {major }}=24.2 \mathrm{~min}, \tau_{\text {minor }}=$ $18.1 \mathrm{~min} ;[\alpha]_{\mathrm{D}}^{25}-48.5\left(\right.$ c $1.0, \mathrm{CHCl}_{3}, 83 \%$ ee $)$. 


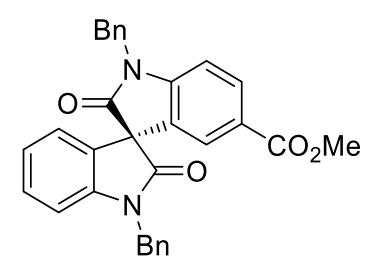

$89.8 \mathrm{mg}, 92 \%$ yield, yellow solid, m.p. $125-126{ }^{\circ} \mathrm{C} .{ }^{1} \mathrm{H}$ NMR $\left(400 \mathrm{MHz}, \mathrm{CDCl}_{3}\right) \delta 8.01$ (dd, $J=8.4$ $\mathrm{Hz}, 1.6 \mathrm{~Hz}, 1 \mathrm{H}), 7.66(\mathrm{~d}, \mathrm{~J}=1.6 \mathrm{~Hz}, 1 \mathrm{H}), 7.26-7.42(\mathrm{~m}, 11 \mathrm{H}), 7.01-7.05(\mathrm{~m}, 1 \mathrm{H}), 6.85-6.94(\mathrm{~m}$, $3 \mathrm{H}), 5.14(\mathrm{~d}, J=15.6 \mathrm{~Hz}, 1 \mathrm{H}), 5.09$ (d, $J=15.6 \mathrm{~Hz}, 1 \mathrm{H}), 5.03(\mathrm{~d}, J=16.0 \mathrm{~Hz}, 1 \mathrm{H}), 4.96$ (d, $J=$ $16.0 \mathrm{~Hz}, 1 \mathrm{H}), 3.83(\mathrm{~s}, 3 \mathrm{H}) ;{ }^{13} \mathrm{C} \mathrm{NMR}\left(100 \mathrm{MHz}, \mathrm{CDCl}_{3}\right) \delta 172.7,171.9,166.3,148.5,144.4,135.0$, 134.7, 132.2, 130.0, 129.1, 129.0, 128.1, 128.0, 127.9, 127.1, 125.4, 124.0, 123.7, 110.3, 110.0, 62.1, 52.2, 44.5, 44.4; ESI-MS $m / z 489.1\left(\mathrm{M}+\mathrm{H}^{+}\right)$, HRMS calcd for $\mathrm{C}_{31} \mathrm{H}_{25} \mathrm{~N}_{2} \mathrm{O}_{4}{ }^{+}(\mathrm{M}+\mathrm{H})^{+}$489.1809, found 489.1814; HPLC Chiralcel OD-H (hexane $/ \mathrm{i}-\mathrm{PrOH}=70: 30,1.0 \mathrm{ml} / \mathrm{min}$ ) $\tau_{\text {major }}=15.4 \mathrm{~min}$, $\tau_{\text {minor }}=22.8 \mathrm{~min} ;[\alpha]_{\mathrm{D}}^{25}-29.0\left(c 1.0, \mathrm{CHCl}_{3}, 91 \% e e\right)$.

(S)-5-acetyl-1,1'-dibenzyl-3,3'-spirobi[indoline]-2,2'-dione (4h)<smiles>CC(=O)c1ccc2c(c1)C(C(=O)Nc1ccccc1)c1ccccc1N2Cc1ccccc1</smiles>

$90.6 \mathrm{mg}, 96 \%$ yield, white solid, m.p. $217-218^{\circ} \mathrm{C} .{ }^{1} \mathrm{H}$ NMR (400 MHz, $\left.\mathrm{CDCl}_{3}\right) \delta 7.90$ (dd, J = 8.4 $\mathrm{Hz}, 2.0 \mathrm{~Hz}, 1 \mathrm{H}), 7.57(\mathrm{~d}, \mathrm{~J}=2.0 \mathrm{~Hz}, 1 \mathrm{H}), 7.27-7.40(\mathrm{~m}, 11 \mathrm{H}), 7.00-7.05(\mathrm{~m}, 1 \mathrm{H}), 6.84-6.92(\mathrm{~m}$, 3H), 4.92-5.16 (m, 4H), 2.47 (s, 3H); ${ }^{13} \mathrm{C}$ NMR $\left(100 \mathrm{MHz}, \mathrm{CDCl}_{3}\right) \delta 196.4,172.7,171.9,148.6$, 144.4, 134.9, 134.6, 132.9, 131.4, 130.0, 129.1, 129.0, 128.0, 127.9, 127.1, 124.0, 123.9, 123.7, 110.3, 109.5, 62.1, 44.5, 44.4, 26.4; ESI-MS $m / z$ 473.1 $\left(\mathrm{M}+\mathrm{H}^{+}\right)$, HRMS calcd for $\mathrm{C}_{31} \mathrm{H}_{25} \mathrm{~N}_{2} \mathrm{O}_{3}{ }^{+}(\mathrm{M}$ $+\mathrm{H})^{+} 473.1860$, found 473.1855; HPLC Chiralcel OD-H (hexane $/ \mathrm{i}-\mathrm{PrOH}=60: 40,1.0 \mathrm{ml} / \mathrm{min}$ ) $\tau_{\text {major }}$ $=13.6 \mathrm{~min}, \tau_{\text {minor }}=19.2 \mathrm{~min} ;[\alpha]_{\mathrm{D}}{ }^{25}-66.4\left(c 1.0, \mathrm{CHCl}_{3}, 86 \%\right.$ ee $)$.

(S)-5-acetyl-1,1'-dibenzyl-5'-methyl-3,3'-spirobi[indoline]-2,2'-dione (4i)<smiles>CC(=O)c1ccc2c(c1)C(C(=O)Nc1ccccc1)(C(=O)Nc1ccccc1)c1cc(C)ccc1N2</smiles>

$89.4 \mathrm{mg}, 92 \%$ yield, yellow solid, m.p. $158-159^{\circ} \mathrm{C} .{ }^{1} \mathrm{H}$ NMR $\left(400 \mathrm{MHz}, \mathrm{CDCl}_{3}\right) \delta 7.90$ (dd, $J=8.4$ $\mathrm{Hz}, 2.0 \mathrm{~Hz}, 1 \mathrm{H}), 7.57(\mathrm{~d}, J=2.0 \mathrm{~Hz}, 1 \mathrm{H}), 7.27-7.40(\mathrm{~m}, 10 \mathrm{H}), 7.07$ (dd, $J=8.0,0.8 \mathrm{~Hz}, 1 \mathrm{H}), 6.87$ $(\mathrm{d}, J=8.0 \mathrm{~Hz}, 1 \mathrm{H}), 6.71-6.74(\mathrm{~m}, 2 \mathrm{H}), 4.92-5.16(\mathrm{~m}, 4 \mathrm{H}), 2.48(\mathrm{~s}, 3 \mathrm{H}), 2.23(\mathrm{~s}, 3 \mathrm{H}) ;{ }^{13} \mathrm{C}$ NMR $(100$ $\left.\mathrm{MHz}, \mathrm{CDCl}_{3}\right) \delta 196.5,172.9,171.8,148.6,142.0,135.0,134.6,133.4,132.9,131.3,130.3,129.1$, 129.0, 128.5, 128.0, 127.8, 127.1, 127.0, 126.9, 124.7, 124.0, 110.0, 109.5, 62.2, 44.5, 44.4, 26.4, 21.1; ESI-MS $m / z$ 487.1 $\left(\mathrm{M}+\mathrm{H}^{+}\right)$, HRMS calcd for $\mathrm{C}_{32} \mathrm{H}_{27} \mathrm{~N}_{2} \mathrm{O}_{3}{ }^{+}(\mathrm{M}+\mathrm{H})^{+}$487.2016, found 487.2014; HPLC Chiralcel OJ-H (hexane $/ \mathrm{i}-\mathrm{PrOH}=70: 30,1.0 \mathrm{ml} / \mathrm{min}$ ) $\tau_{\text {major }}=15.3 \mathrm{~min}, \tau_{\text {minor }}=$ $28.9 \min ;[\alpha]_{\mathrm{D}}{ }^{25}-64.4\left(c 1.0, \mathrm{CHCl}_{3}, 85 \% e e\right)$. 


\section{Computational Results}

\section{Computational methods}

All the density functional theory (DFT) computations were performed with Gaussian 16 program package. ${ }^{3}$ Calculations were carried out with B3LYP. ${ }^{4}$ The geometry optimization and frequency calculations were conducted with the basis set $6-31 \mathrm{G}(\mathrm{d})^{5-6}$ for all the atoms except $\mathrm{Cu}$ and I atoms, for which the Lanl2dz with effective core potential (ECP) was adopted and the polarization functions were added for $\mathrm{I}(\xi(\mathrm{d})=0.289)$ and $\mathrm{Cu}(\xi(\mathrm{f})=3.525)^{7-9}$. Single point energies were calculated with larger basis sets, Def2TZVP ${ }^{10}$ and $6-311+\mathrm{G}^{* * 11-13}$. Solvent effect was conducted with self-consistent reaction field (SCRF) method based on $\mathrm{SMD}^{14}$ model in dioxane. Frequency analysis was performed to obtain thermal corrections at $298.15 \mathrm{~K}$ and to confirm the transition states with only one imaginary frequency. Some structures shown in figures were generated with the CYLview. ${ }^{15}$

2. Computational results

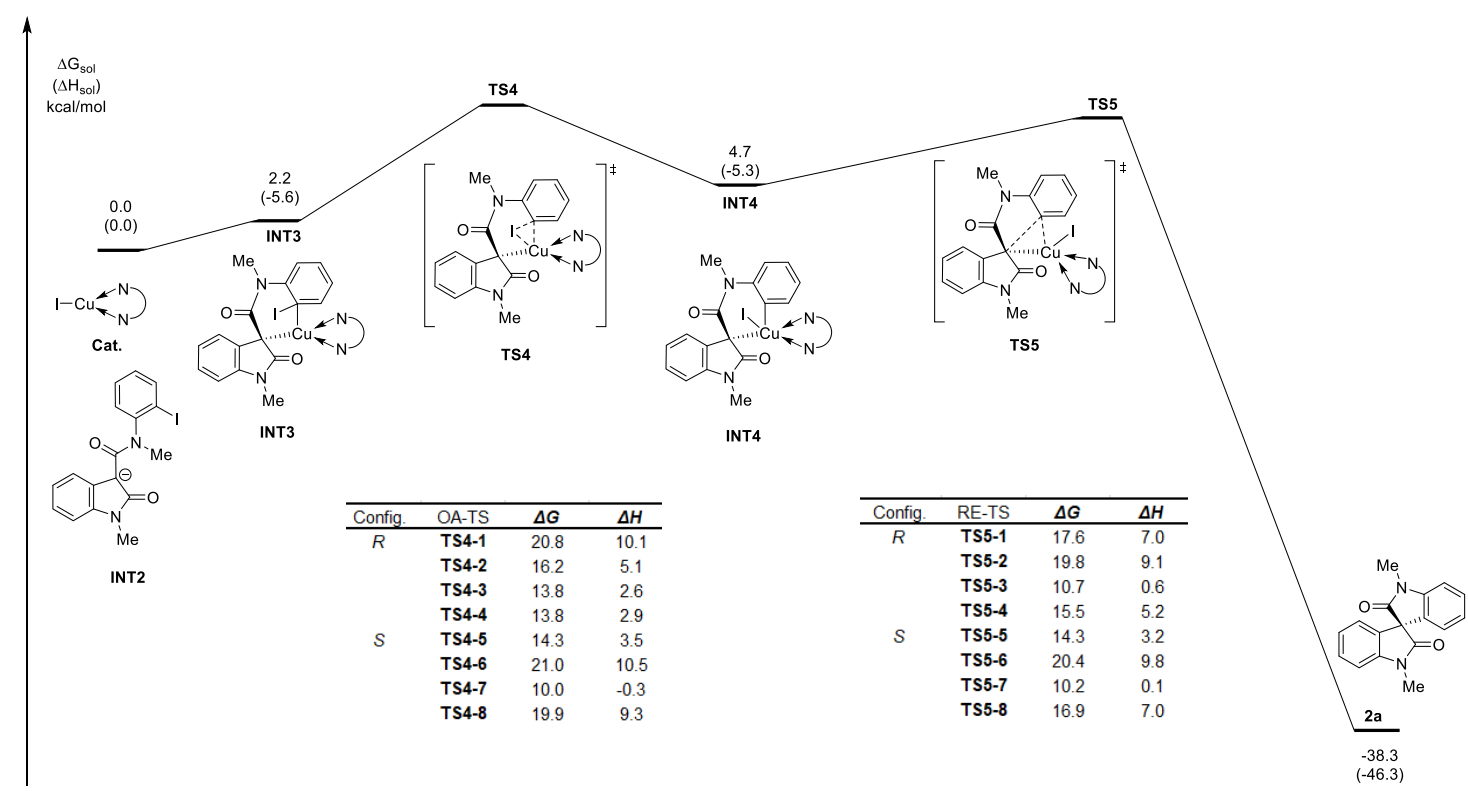

Figure S1. Potential Energy Surface. Free energies and enthalpies (in parenthesis) are given in $\mathrm{kcal} / \mathrm{mol}$. 


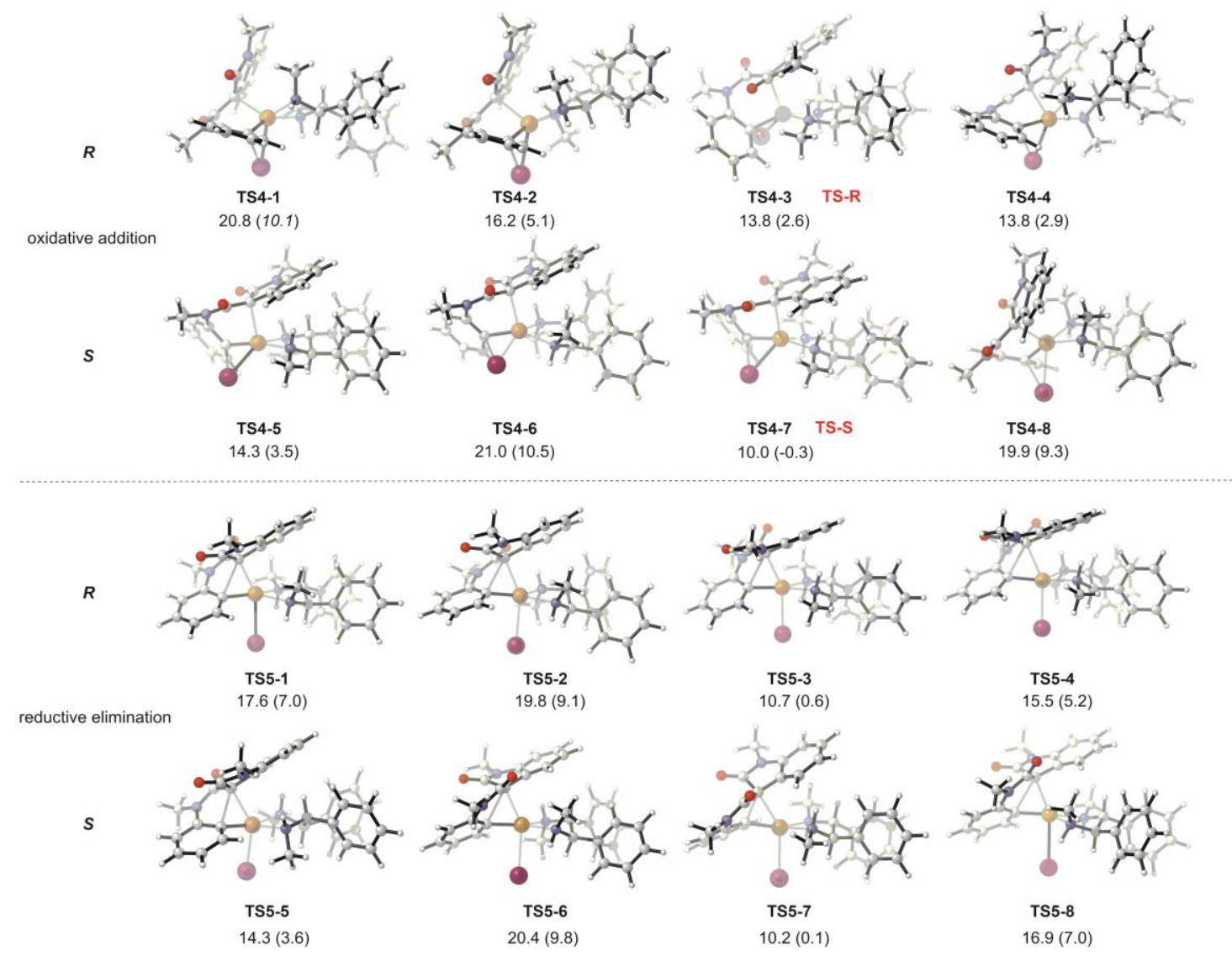

Figure S2. Conformation search of transition states, $\Delta \Delta \mathrm{G},(\Delta \Delta \mathrm{H})$ relative to TS4-7. Free energies and enthalpies (in parenthesis) are given in $\mathrm{kcal} / \mathrm{mol}$. 
Table S1. Energies of transition states. (units of $H$ and $G$ are Hartree)

\begin{tabular}{ccc}
\hline & $\boldsymbol{H}$ & $\boldsymbol{G}$ \\
\hline TS4-1 & -3585.780844 & -3585.892442 \\
TS4-2 & -3585.788855 & -3585.899849 \\
TS4-3 & -3585.792812 & -3585.903644 \\
TS4-4 & -3585.792397 & -3585.903602 \\
TS4-5 & -3585.791303 & -3585.902765 \\
TS4-6 & -3585.780160 & -3585.892081 \\
TS4-7 & -3585.797495 & -3585.909659 \\
TS4-8 & -3585.782134 & -3585.893879 \\
TS5-1 & -3585.785786 & -3585.897519 \\
TS5-2 & -3585.782446 & -3585.894082 \\
TS5-3 & -3585.795997 & -3585.908524 \\
TS5-4 & -3585.788700 & -3585.900933 \\
TS5-5 & -3585.791800 & -3585.902793 \\
TS5-6 & -3585.781403 & -3585.893192 \\
TS5-7 & -3585.796863 & -3585.909407 \\
TS5-8 & -3585.785726 & -3585.898733 \\
Cat & -2669.625983 & -2669.703309 \\
INT1 & -1214.609650 & -1214.681752 \\
INT2 & -1214.115683 & -1214.186237 \\
INT3 & -3585.805891 & -3585.922044 \\
INT4 & -3585.805422 & -3585.918113 \\
$\mathbf{2 a}$ & -916.232126 & -916.295227 \\
I & -297.944713 & -297.963921 \\
\hline & &
\end{tabular}




\section{Cartesian Coordinates}

\section{TS4-1}

C $\quad 2.146045 \quad 2.411678 \quad-0.271952$

C $\quad 1.622352 \quad 2.948053 \quad-1.475545$

$\begin{array}{llll}\text { C } & 2.293164 & 3.251264 & 0.834739\end{array}$

$\begin{array}{llll}\text { C } & 1.172310 & 4.261478 & -1.569998\end{array}$

$\begin{array}{llll}\text { C } & 1.864865 & 4.581111 & 0.744511\end{array}$

$\mathrm{H} \quad 2.745620 \quad 2.865200 \quad 1.739894$

$\begin{array}{llll}\text { C } & 1.295017 & 5.076265 & -0.434171\end{array}$

$\begin{array}{llll}\mathrm{H} & 0.769739 & 4.652168 & -2.500270\end{array}$

$\mathrm{H} \quad 1.976065 \quad 5.237322 \quad 1.603506$

H $\quad 0.960922 \quad 6.109321 \quad-0.481976$

C $\quad 2.285576 \quad 0.793537 \quad-1.971662$

$\begin{array}{lllll}\text { O } & 2.533294 & -0.175039 & -2.691228\end{array}$

$\begin{array}{llll}\mathrm{N} & 1.692623 & 1.976770 & -2.466130\end{array}$

$\begin{array}{llll}\text { C } & 1.438440 & 2.203899 & -3.868581\end{array}$

$\mathrm{H} \quad 2.022556 \quad 3.056633 \quad-4.236341$

$\mathrm{H} \quad 0.375116 \quad 2.402849 \quad-4.056999$

H $\quad 1.738054 \quad 1.301625 \quad-4.405427$

$\begin{array}{llll}\text { C } & 3.590719 & 0.402776 & 0.256232\end{array}$

$\begin{array}{lllll}\text { C } & 3.003264 & -1.898521 & -0.422251\end{array}$

$\begin{array}{llll}\text { C } & 2.586389 & -3.959249 & -1.662086\end{array}$

$\begin{array}{llll}\mathrm{O} & 4.258126 & 1.056882 & 1.056978\end{array}$

$\begin{array}{llll}\mathrm{N} & 3.917875 & -0.936088 & 0.046202\end{array}$

$\begin{array}{llll}\text { C } & 1.657599 & -1.908672 & -0.035142\end{array}$

C $\quad 1.270472 \quad-3.995136-1.196424$

H $\quad 2.956935 \quad-4.742310 \quad-2.317206$

$\begin{array}{lllll}\text { C } & 5.097440 & -1.410845 & 0.782200\end{array}$

$\begin{array}{lllll}\text { C } & 0.813013 & -2.972488 & -0.362620\end{array}$

H $\quad 0.610077 \quad-4.819834-1.449886$

H $\quad 5.338644 \quad-2.423491 \quad 0.457920$

H $\quad 4.903159 \quad-1.410685 \quad 1.860877$

$\begin{array}{llll}\text { H } & 5.942729 & -0.749043 & 0.586497\end{array}$

H $\quad-0.184086 \quad-3.019677 \quad 0.063305$

C $\quad 2.475474 \quad 1.005150 \quad-0.513327$

$\begin{array}{lllll}\text { C } & 3.433034 & -2.922105 & -1.282681\end{array}$

H $\quad 4.446688 \quad-2.871726 \quad-1.667986$

I $\quad \begin{array}{llll}1.140693 & -1.327532 & 2.522523\end{array}$

$\begin{array}{llll}\mathrm{Cu} & 0.712405 & -0.116790 & 0.157194\end{array}$

$\mathrm{N} \quad-0.892233 \quad-0.649417 \quad-1.308902$

$\begin{array}{llll}\text { C } & -2.123374 & -0.611444 & -0.478481\end{array}$

$\begin{array}{llll}\text { C } & -2.235563 & 0.781214 & 0.181799\end{array}$

$\begin{array}{llll}\mathrm{N} & -0.984960 & 1.046641 & 0.947065\end{array}$
H $\quad-1.059080 \quad 0.544373 \quad 1.834168$

$\begin{array}{llll}\text { C } & -0.822699 & 2.477918 & 1.269843\end{array}$

$\mathrm{H} \quad-0.595791 \quad 3.030355 \quad 0.356202$

$\mathrm{H} \quad 0.025707 \quad 2.586475 \quad 1.943526$

H $\quad-1.723372 \quad 2.894117 \quad 1.740132$

C $\quad \begin{array}{llll}-3.476946 & 0.915969 & 1.047307\end{array}$

$\begin{array}{llll}\text { C } & -3.706115 & 0.024934 & 2.105728\end{array}$

$\begin{array}{llll}\text { C } & -4.394222 & 1.948216 & 0.825739\end{array}$

C $\quad-4.831556 \quad 0.160827 \quad 2.917345$

$\mathrm{H} \quad-3.003432 \quad-0.782367 \quad 2.302163$

$\begin{array}{llll}\text { C } & -5.523125 & 2.087322 & 1.635843\end{array}$

$\mathrm{H} \quad-4.224315 \quad 2.648253 \quad 0.010823$

$\begin{array}{llll}\text { C } & -5.744959 & 1.192329 & 2.683192\end{array}$

H $\quad-4.996276 \quad-0.539008 \quad 3.731924$

H $\quad-6.225408 \quad 2.895168 \quad 1.449345$

$\mathrm{H} \quad-6.622130 \quad 1.297776 \quad 3.315435$

$\mathrm{H} \quad-2.271120 \quad 1.542179 \quad-0.603830$

C $\quad-3.369939-1.074205-1.213533$

C $\quad-4.154531-0.212818-1.992882$

C $\quad-3.719501-2.429638-1.157843$

C $\quad-5.257147 \quad-0.696190 \quad-2.698556$

H $\quad-3.913788 \quad 0.844527 \quad-2.044723$

C $\quad-4.820808 \quad-2.917807-1.861456$

H $\quad-3.124709-3.109266-0.550086$

$\begin{array}{llll}\text { C } & -5.593337 & -2.050001 & -2.635933\end{array}$

H $\quad-5.855884 \quad-0.012992 \quad-3.294744$

H $\quad-5.078192-3.971655-1.799968$

H $\quad-6.453759-2.424841 \quad-3.183195$

H $\quad-1.928319 \quad-1.327625 \quad 0.330247$

C $\quad-0.903836 \quad 0.127931 \quad-2.558836$

H $\quad 0.016938 \quad-0.111138 \quad-3.092484$

$\mathrm{H} \quad-0.892084 \quad 1.195363 \quad-2.329441$

H $\quad-1.770088-0.100016 \quad-3.192566$

H $\quad-0.693190 \quad-1.618545 \quad-1.547922$

\section{TS4-2}

$\begin{array}{llll}\text { C } & 0.530454 & 2.587061 & -0.696168\end{array}$

C $\quad-0.324575 \quad 2.542833 \quad-1.828387$

C $\quad 0.260186 \quad 3.517040 \quad 0.313703$

C $\quad-1.426025 \quad 3.382883-1.964300$

$\begin{array}{llll}\text { C } & -0.848454 & 4.367787 & 0.186264\end{array}$

$\mathrm{H} \quad 0.931204 \quad 3.588982 \quad 1.161628$

$\begin{array}{llll}\text { C } & -1.679961 & 4.304895 & -0.937080\end{array}$ 


\begin{tabular}{|c|c|c|c|}
\hline $\mathrm{H}$ & -2.056597 & 3.340170 & -2.847862 \\
\hline $\mathrm{H}$ & -1.053726 & 5.095272 & 0.966747 \\
\hline $\mathrm{H}$ & -2.528877 & 4.978135 & -1.022122 \\
\hline $\mathrm{C}$ & 1.371012 & 1.030448 & -2.235984 \\
\hline $\mathrm{O}$ & 2.045978 & 0.259210 & -2.916710 \\
\hline$\Lambda$ & 0.157359 & 1.587406 & -2.710914 \\
\hline $\mathrm{C}$ & -0.336960 & 1.362484 & -4.048476 \\
\hline $\mathrm{H}$ & -0.311992 & 2.286329 & -4.640444 \\
\hline $\mathrm{H}$ & -1.369288 & 0.988223 & -4.031610 \\
\hline $\mathrm{H}$ & 0.312521 & 0.617753 & -4.512677 \\
\hline C & 2.877327 & 1.750416 & -0.218534 \\
\hline C & 3.607832 & -0.553402 & -0.737297 \\
\hline $\mathrm{C}$ & 4.171079 & -2.561692 & -1.994711 \\
\hline $\mathrm{O}$ & 3.125352 & 2.738373 & 0.474872 \\
\hline $\mathrm{N}$ & 3.877012 & 0.795329 & -0.417898 \\
\hline $\mathrm{C}$ & 2.592349 & -1.279603 & -0.094634 \\
\hline C & 3.178685 & -3.279743 & -1.323497 \\
\hline $\mathrm{H}$ & 4.784584 & -3.048938 & -2.746905 \\
\hline C & 5.182879 & 1.105160 & 0.175696 \\
\hline $\mathrm{C}$ & 2.402996 & -2.641311 & -0.355900 \\
\hline $\mathrm{H}$ & 3.025406 & -4.336592 & -1.523751 \\
\hline $\mathrm{H}$ & 5.151005 & 1.003496 & 1.267232 \\
\hline $\mathrm{H}$ & 5.456144 & 2.132924 & -0.066404 \\
\hline $\mathrm{H}$ & 5.928847 & 0.418941 & -0.228755 \\
\hline $\mathrm{H}$ & 1.691733 & -3.210125 & 0.232881 \\
\hline $\mathrm{C}$ & 1.558435 & 1.557796 & -0.864854 \\
\hline $\mathrm{C}$ & 4.372065 & -1.215267 & -1.707198 \\
\hline $\mathrm{H}$ & 5.106487 & -0.636707 & -2.258581 \\
\hline I & 2.402292 & -0.875112 & 2.408893 \\
\hline $\mathrm{Cu}$ & 0.888539 & -0.211059 & 0.355803 \\
\hline $\mathrm{N}$ & -0.583837 & -1.651497 & -0.449694 \\
\hline $\mathrm{C}$ & -1.887151 & -1.393880 & 0.231870 \\
\hline $\mathrm{C}$ & -2.037057 & 0.132552 & 0.419311 \\
\hline $\mathrm{N}$ & -0.878805 & 0.627600 & 1.228908 \\
\hline
\end{tabular}

$\begin{array}{llll}\mathrm{C} & -3.378979 & 0.587235 & 0.970338 \\ \mathrm{C} & -4.123070 & -0.176161 & 1.880259 \\ \mathrm{C} & -3.860203 & 1.849138 & 0.598967 \\ \mathrm{C} & -5.322813 & 0.309052 & 2.401723 \\ \mathrm{H} & -3.777009 & -1.161050 & 2.178945 \\ \mathrm{C} & -5.058697 & 2.337543 & 1.120778 \\ \mathrm{H} & -3.286024 & 2.454303 & -0.096952 \\ \mathrm{C} & -5.795011 & 1.567868 & 2.023417 \\ \mathrm{H} & -5.889657 & -0.298515 & 3.102002 \\ \mathrm{H} & -5.416334 & 3.318611 & 0.819570 \\ \mathrm{H} & -6.730448 & 1.944204 & 2.428514 \\ \mathrm{H} & -1.891884 & 0.607973 & -0.556321 \\ \mathrm{C} & -3.056647 & -2.082342 & -0.451023 \\ \mathrm{C} & -3.799864 & -1.469466 & -1.468734 \\ \mathrm{C} & -3.363651 & -3.402850 & -0.099625 \\ \mathrm{C} & -4.824034 & -2.160818 & -2.115790 \\ \mathrm{H} & -3.590891 & -0.442203 & -1.751129 \\ \mathrm{C} & -4.386897 & -4.098974 & -0.744593 \\ \mathrm{H} & -2.797861 & -3.889940 & 0.692965 \\ \mathrm{C} & -5.120730 & -3.477667 & -1.756826 \\ \mathrm{H} & -5.393858 & -1.668143 & -2.898849 \\ \mathrm{H} & -4.613239 & -5.121108 & -0.453477 \\ \mathrm{H} & -5.920917 & -4.013736 & -2.259531 \\ \mathrm{H} & -1.767430 & -1.837566 & 1.226311 \\ \mathrm{C} & -0.591611 & -1.589759 & -1.925750 \\ \mathrm{H} & 0.441763 & -1.640257 & -2.273647 \\ \mathrm{H} & -0.995836 & -0.627194 & -2.237379 \\ \mathrm{H} & -1.189904 & -2.388196 & -2.379173 \\ \mathrm{H} & -0.267641 & -2.579153 & -0.179631 \\ \mathrm{H} & -0.785088 & 1.626490 & 1.033905 \\ \mathrm{C} & -0.992922 & 0.465906 & 2.689983 \\ \mathrm{H} & -1.890251 & 0.946886 & 3.093916 \\ \mathrm{H} & -0.102426 & 0.904085 & 3.143787 \\ \mathrm{H} & -1.004305 & -0.595195 & 2.951409\end{array}$

$\begin{array}{llll}\text { C } & -3.378979 & 0.587235 & 0.970338\end{array}$

$\begin{array}{llll}\text { C } & -4.123070 & -0.176161 & 1.880259\end{array}$

$\begin{array}{lllll}\mathrm{C} & -3.860203 & 1.849138 & 0.598967\end{array}$

$\begin{array}{llll}\text { C } & -5.322813 & 0.309052 & 2.401723\end{array}$

H $\quad-3.777009 \quad-1.161050 \quad 2.178945$

$\begin{array}{llll}\text { C } & -5.058697 & 2.337543 & 1.120778\end{array}$

$\begin{array}{lll}-3.286024 & 2.454303 & -0.096952\end{array}$

$\begin{array}{lllll}\text { C } & -5.795011 & 1.567868 & 2.023417\end{array}$

H $\quad-5.889657 \quad-0.298515 \quad 3.102002$

$\begin{array}{lll}-5.416334 & 3.318611 & 0.819570\end{array}$

$\mathrm{H} \quad-6.730448 \quad 1.944204 \quad 2.428514$

C $-3.056647-2.082342-0.451023$

C $-3.056647-2.082342-0.451023$

$\begin{array}{llll}\text { C } & -3.363651 & -3.402850 & -0.099625\end{array}$

$\begin{array}{llll}\text { C } & -4.824034 & -2.160818 & -2.115790\end{array}$

H $\quad-3.590891 \quad-0.442203 \quad-1.751129$

$\begin{array}{lllll}\text { C } & -4.386897 & -4.098974 & -0.744593\end{array}$

H $\quad-2.797861 \quad-3.8899400 .692965$

H $\quad-5.393858-1.668143 \quad-2.898849$

$\mathrm{H} \quad-4.613239 \quad-5.121108 \quad-0.453477$

(1)

$\begin{array}{llll}-1.767430 & -1.837566 & 1.226311\end{array}$

C $\quad-0.591611-1.589759-1.925750$

$\begin{array}{llll}0.441763 & -1.640257 & -2.273647\end{array}$

H $\quad-0.995836-0.627194 \quad-2.237379$

H $\quad-0.267641-2.579153 \quad-0.179631$

$\mathrm{H} \quad-0.785088 \quad 1.626490 \quad 1.033905$

$\begin{array}{llll}-1.890251 & 0.94686 & 3.093916\end{array}$

$\mathrm{H} \quad-0.102426 \quad 0.904085 \quad 3.143787$

$\begin{array}{llll}-1.004305 & -0.595195 & 2.951409\end{array}$ 


\section{TS4-3}

$\begin{array}{lllll}\text { C } & 0.267004 & -0.812771 & -2.404089\end{array}$

$\begin{array}{llll}\text { C } & -0.652567 & -1.863283 & -2.146230\end{array}$

$\begin{array}{lllll}\text { C } & -0.042468 & 0.112284 & -3.405909\end{array}$

$\begin{array}{lllll}\text { C } & -1.868745 & -1.974419 & -2.813772\end{array}$

$\begin{array}{lllll}\text { C } & -1.268381 & 0.015170 & -4.078180\end{array}$

$\mathrm{H} \quad 0.681218 \quad 0.876632 \quad-3.660808$

$\begin{array}{llll}\text { C } & -2.178354 & -1.005905 & -3.781049\end{array}$

$\mathrm{H} \quad-2.544275 \quad-2.801314 \quad-2.612487$

$\mathrm{H} \quad-1.508020 \quad 0.737961 \quad-4.853502$

H $\quad-3.118269-1.072447 \quad-4.322246$

$\begin{array}{llll}\text { C } & 1.216738 & -2.288155 & -0.848668\end{array}$

$\begin{array}{lllll}\text { O } & 1.939705 & -2.962644 & -0.118165\end{array}$

$\mathrm{N} \quad-0.113349-2.696828 \quad-1.169556$

$\begin{array}{lllll}\text { C } & -0.633470 & -3.999210 & -0.807761\end{array}$

H $\quad-0.750677 \quad-4.637438 \quad-1.692721$

$\mathrm{H} \quad-1.605015 \quad-3.908436 \quad-0.306758$

H $\quad 0.087121 \quad-4.454895 \quad-0.125794$

$\begin{array}{llll}\text { C } & 2.726534 & -0.407070 & -1.865726\end{array}$

$\begin{array}{lllll}\text { C } & 3.657832 & -0.829332 & 0.386321\end{array}$

$\begin{array}{llll}\text { C } & 4.347039 & -1.984751 & 2.415149\end{array}$

$\begin{array}{llll}\text { O } & 2.903020 & 0.221983 & -2.911129\end{array}$

$\begin{array}{lllll}\mathrm{N} & 3.808259 & -0.592980 & -0.997141\end{array}$

$\begin{array}{lllll}\text { C } & 2.712808 & -0.134845 & 1.157620\end{array}$

$\begin{array}{llll}\text { C } & 3.409683 & -1.272625 & 3.165937\end{array}$

$\mathrm{H} \quad 4.987980 \quad-2.717382 \quad 2.897171$

$\begin{array}{llll}\text { C } & 5.097916 & -0.075632 & -1.467902\end{array}$

$\begin{array}{lllll}\text { C } & 2.603959 & -0.321930 & 2.536420\end{array}$

$\begin{array}{lllll}\mathrm{H} & 3.322526 & -1.426722 & 4.237877\end{array}$

H $\quad 5.266802 \quad-0.400278-2.495492$

$\mathrm{H} \quad 5.891296 \quad-0.458697 \quad-0.824044$

$\mathrm{H} \quad 5.110878 \quad 1.020701 \quad-1.445186$

$\mathrm{H} \quad 1.917240 \quad 0.288969 \quad 3.111123$

$\begin{array}{lllll}\text { C } & 1.414048 & -0.985967 & -1.512251\end{array}$

$\begin{array}{llll}\text { C } & 4.458667 & -1.771524 & 1.043154\end{array}$

$\begin{array}{llll}\mathrm{H} & 5.144509 & -2.365730 & 0.447359\end{array}$

I $\quad 2.631298 \quad 2.394168 \quad 0.686373$

$\begin{array}{llll}\mathrm{Cu} & 0.977853 & 0.393541 & 0.255333\end{array}$

$\begin{array}{lllll}\mathrm{N} & -0.734969 & 1.652795 & -0.043565\end{array}$

$\begin{array}{llll}\mathrm{C} & -1.964323 & 0.820051 & 0.022495\end{array}$

$\begin{array}{llll}\mathrm{C} & -1.882571 & -0.062511 & 1.293686\end{array}$

$\begin{array}{lllll}\mathrm{N} & -0.637047 & -0.858579 & 1.221366\end{array}$

$\begin{array}{llll}\mathrm{H} & -0.734481 & -1.519103 & 0.446669\end{array}$ $\begin{array}{llll}\text { C } & -0.336915 & -1.647862 & 2.424985\end{array}$

$\begin{array}{llll}\mathrm{H} & -0.154705 & -0.972940 & 3.266891\end{array}$

$\begin{array}{lllll}\mathrm{H} & 0.573768 & -2.217712 & 2.231207\end{array}$

$\begin{array}{lllll}\mathrm{H} & -1.154365 & -2.329738 & 2.694700\end{array}$

$\begin{array}{llll}\text { C } & -3.123127 & -0.918377 & 1.488546\end{array}$

$\begin{array}{lllll}\text { C } & -3.554885 & -1.783981 & 0.473514\end{array}$

$\begin{array}{llll}\text { C } & -3.827399 & -0.891424 & 2.695653\end{array}$

$\begin{array}{lllll}\text { C } & -4.665858 & -2.604049 & 0.665083\end{array}$

$\mathrm{H} \quad-3.031108 \quad-1.806360-0.477105$

$\begin{array}{llll}\text { C } & -4.941901 & -1.710833 & 2.890993\end{array}$

$\mathrm{H} \quad-3.503580 \quad-0.221570 \quad 3.489091$

$\begin{array}{lllll}\text { C } & -5.363019 & -2.570984 & 1.876186\end{array}$

H $\quad-4.991662-3.264697 \quad-0.134102$

$\begin{array}{llll}\mathrm{H} & -5.478694 & -1.676120 & 3.835160\end{array}$

$\begin{array}{llll}\mathrm{H} & -6.229608 & -3.209158 & 2.025254\end{array}$

$\begin{array}{lllll}\mathrm{H} & -1.775821 & 0.599528 & 2.163889\end{array}$

$\begin{array}{llll}\text { C } & -3.249326 & 1.623774 & -0.043544\end{array}$

$\begin{array}{llll}\text { C } & -3.582994 & 2.539928 & 0.962422\end{array}$

$\begin{array}{llll}\text { C } & -4.112460 & 1.468606 & -1.133222\end{array}$

$\begin{array}{llll}\text { C } & -4.761455 & 3.281667 & 0.884826\end{array}$

$\begin{array}{llll}\mathrm{H} & -2.921204 & 2.678122 & 1.815438\end{array}$

$\begin{array}{llll}\text { C } & -5.294339 & 2.207594 & -1.212641\end{array}$

$\begin{array}{llll}\mathrm{H} & -3.849612 & 0.767562 & -1.922113\end{array}$

$\begin{array}{llll}\text { C } & -5.622192 & 3.114727 & -0.203518\end{array}$

$\mathrm{H} \quad-5.009203 \quad 3.987938 \quad 1.672672$

$\mathrm{H} \quad-5.956601 \quad 2.076343 \quad-2.064124$

H $\quad-6.5412293 .691158 \quad-0.264218$

$\mathrm{H} \quad-1.915251 \quad 0.166519 \quad-0.854295$

$\begin{array}{llll}\text { C } & -0.701508 & 2.575318 & -1.198197\end{array}$

H $\quad 0.275312 \quad 3.062678 \quad-1.218421$

$\mathrm{H} \quad-1.494276 \quad 3.329916 \quad-1.142086$

$\mathrm{H} \quad-0.825923 \quad 1.993112 \quad-2.109983$

$\begin{array}{llll}\mathrm{H} & -0.687484 & 2.224324 & 0.803208\end{array}$

\section{TS4-4}

$\begin{array}{llll}\text { C } & -0.219326 & 0.028618 & -2.445410\end{array}$

$\begin{array}{lllll}\text { C } & 0.724689 & 1.083671 & -2.562442\end{array}$

$\begin{array}{lllll}\text { C } & 0.071313 & -1.205739 & -3.036023\end{array}$

$\begin{array}{llll}\text { C } & 1.925290 & 0.935060 & -3.249712\end{array}$

$\begin{array}{lllll}\text { C } & 1.282660 & -1.366917 & -3.723460\end{array}$

$\begin{array}{lllll}\mathrm{H} & -0.664169 & -2.001070 & -2.993662\end{array}$

$\begin{array}{llll}\text { C } & 2.197348 & -0.312175 & -3.832688\end{array}$

$\begin{array}{llll}\mathrm{H} & 2.621219 & 1.763313 & -3.348225\end{array}$

H $\quad 1.504846 \quad-2.320277 \quad-4.195193$ 


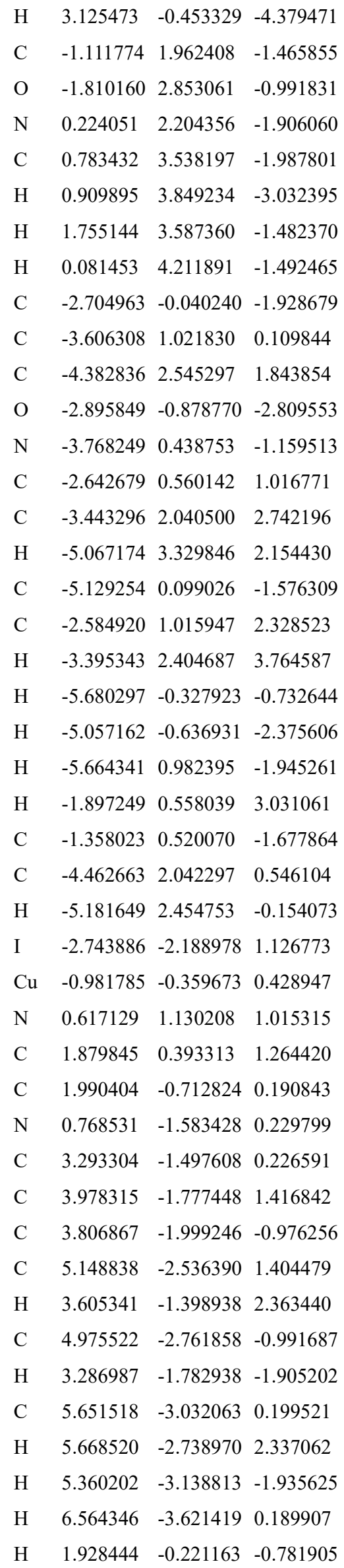

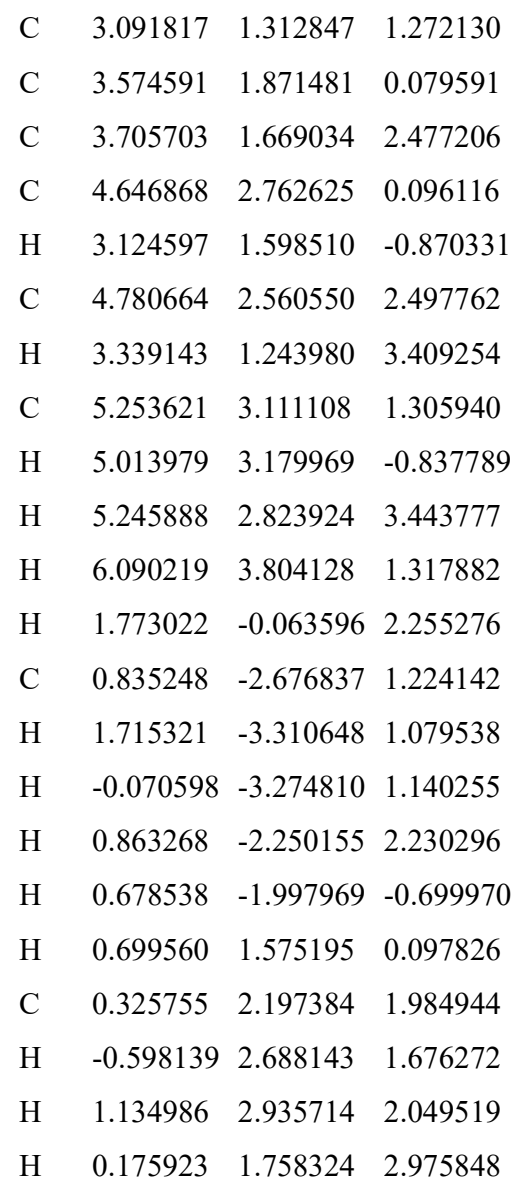

\section{TS4-5}

$\begin{array}{llll}\text { C } & 0.162226 & 0.905679 & -2.382667\end{array}$

$\begin{array}{lllll}\text { C } & -0.740829 & 1.969197 & -2.115227\end{array}$

$\begin{array}{llll}\text { C } & -0.188191 & -0.042137 & -3.351440\end{array}$

$\begin{array}{llll}\text { C } & -1.957886 & 2.099555 & -2.776953\end{array}$

$\begin{array}{llll}\text { C } & -1.412481 & 0.082532 & -4.025619\end{array}$

H $\quad 0.507776 \quad-0.837379-3.590149$

$\begin{array}{lllll}\text { C } & -2.286141 & 1.138731 & -3.746610\end{array}$

$\mathrm{H} \quad-2.625606 \quad 2.929084 \quad-2.561677$

H $\quad-1.679526-0.649886-4.782366$

$\mathrm{H} \quad \begin{array}{llll}-3.227817 & 1.221483 & -4.282468\end{array}$

$\begin{array}{llll}\text { C } & 1.121515 & 2.343408 & -0.800753\end{array}$

$\begin{array}{llll}\text { O } & 1.842669 & 2.988595 & -0.041758\end{array}$

$\mathrm{N} \quad-0.190616 \quad 2.781598 \quad-1.131875$

$\begin{array}{llll}\text { C } & -0.690924 & 4.082346 & -0.745591\end{array}$

$\mathrm{H} \quad-0.862641 \quad 4.714567 \quad-1.625816$

$\begin{array}{lllll}\mathrm{H} & -1.629939 & 4.000775 & -0.184271\end{array}$

$\mathrm{H} \quad 0.068209 \quad 4.543273 \quad-0.110136$

$\begin{array}{llll}\text { C } & 2.619455 & 0.489886 & -1.899439\end{array}$

$\begin{array}{llll}\text { C } & 3.648684 & 0.909177 & 0.308539\end{array}$

$\begin{array}{llll}\text { C } & 4.463095 & 2.017358 & 2.317866\end{array}$ 


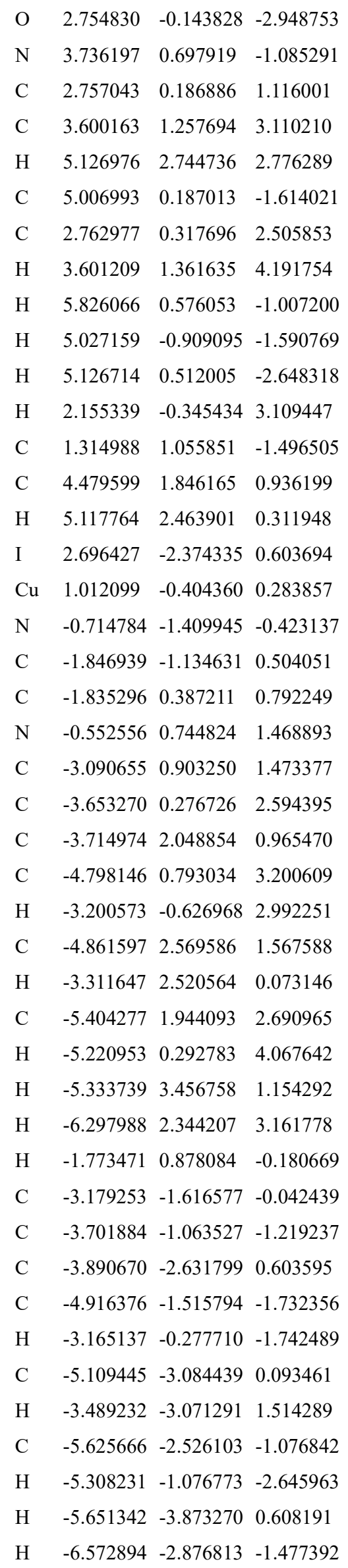

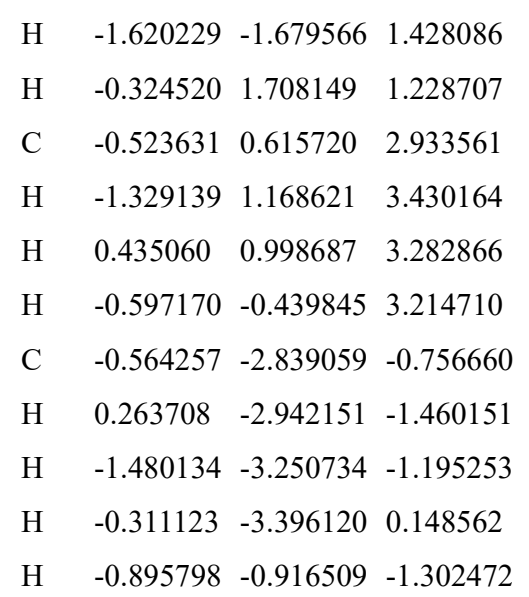

\section{TS4-6}

$\begin{array}{llll}\text { C } & -0.460913 & 1.438100 & 2.155585\end{array}$

$\begin{array}{llll}\text { C } & 0.442574 & 2.434568 & 1.700880\end{array}$

$\begin{array}{lllll}\text { C } & -0.209737 & 0.815411 & 3.381797\end{array}$

$\begin{array}{llll}\text { C } & 1.598823 & 2.767210 & 2.399394\end{array}$

$\begin{array}{llll}\text { C } & 0.950827 & 1.143932 & 4.093778\end{array}$

$\begin{array}{lllll}\text { H } & -0.921549 & 0.095986 & 3.766363\end{array}$

$\begin{array}{llll}\text { C } & 1.853784 & 2.094796 & 3.604649\end{array}$

$\begin{array}{llll}\mathrm{H} & 2.264694 & 3.547989 & 2.042581\end{array}$

$\begin{array}{lllll}\mathrm{H} & 1.148377 & 0.655666 & 5.044277\end{array}$

$\begin{array}{llll}\mathrm{H} & 2.746819 & 2.339570 & 4.173214\end{array}$

$\begin{array}{lllll}\text { C } & -1.341355 & 2.448407 & 0.232112\end{array}$

$\begin{array}{llll}\text { O } & -2.041100 & 2.895176 & -0.672675\end{array}$

$\begin{array}{llll}\mathrm{N} & -0.051773 & 2.982476 & 0.519672\end{array}$

$\begin{array}{lllll}\text { C } & 0.436734 & 4.198916 & -0.096260\end{array}$

$\begin{array}{lllll}\mathrm{H} & 0.492990 & 5.015270 & 0.634951\end{array}$

$\begin{array}{cllll}\mathrm{H} & 1.431558 & 4.048684 & -0.533249\end{array}$

$\begin{array}{lllll}\mathrm{H} & -0.269368 & 4.467485 & -0.884767\end{array}$

$\begin{array}{llll}\text { C } & -2.869501 & 0.830690 & 1.597369\end{array}$

$\begin{array}{lllll}\text { C } & -3.664508 & 0.634951 & -0.729777\end{array}$

$\begin{array}{llll}\text { C } & -4.285150 & 1.124619 & -3.033066\end{array}$

$\begin{array}{llll}\text { O } & -3.100946 & 0.490601 & 2.758885\end{array}$

$\begin{array}{lllll}\mathrm{N} & -3.898571 & 0.766938 & 0.654537\end{array}$

$\begin{array}{llll}\text { C } & -2.635358 & -0.172928 & -1.230985\end{array}$

$\begin{array}{llll}\text { C } & -3.276116 & 0.285622 & -3.508251\end{array}$

$\begin{array}{lllll}\mathrm{H} & -4.932447 & 1.647476 & -3.731346\end{array}$

$\begin{array}{llll}\text { C } & -5.206807 & 0.351740 & 1.174222\end{array}$

$\begin{array}{llll}\text { C } & -2.461205 & -0.391612 & -2.595920\end{array}$

$\begin{array}{lllll}\mathrm{H} & -3.141228 & 0.123956 & -4.574142\end{array}$

$\begin{array}{llll}\mathrm{H} & -5.963846 & 0.513133 & 0.405628\end{array}$

$\begin{array}{llll}\mathrm{H} & -5.194433 & -0.708295 & 1.454232\end{array}$

$\begin{array}{lllll}\mathrm{H} & -5.449586 & 0.939362 & 2.060653\end{array}$ 
$\mathrm{H} \quad-1.720660 \quad-1.104480 \quad-2.940559$

$\begin{array}{llll}\text { C } & -1.542268 & 1.328929 & 1.176642\end{array}$

$\begin{array}{llll}\text { C } & -4.469215 & 1.301179 & -1.663218\end{array}$

$\mathrm{H} \quad-5.221760 \quad 1.991038 \quad-1.294036$

I $\quad-2.539401-2.549611-0.018885$

$\mathrm{Cu} \quad-0.943178-0.427935-0.188546$

$\begin{array}{llll}\mathrm{N} & 0.769597 & -1.648669 & 0.353225\end{array}$

$\begin{array}{llll}\text { C } & 1.966505 & -1.272113 & -0.467539\end{array}$

$\begin{array}{llll}\text { C } & 1.948274 & 0.262662 & -0.640330\end{array}$

$\mathrm{N} \quad 0.696709 \quad 0.633650 \quad-1.364591$

C $\quad 3.205004 \quad 0.860253 \quad-1.248353$

$\begin{array}{llll}\text { C } & 3.872438 & 0.271283 & -2.331179\end{array}$

$\begin{array}{llll}\text { C } & 3.697490 & 2.066751 & -0.734798\end{array}$

$\begin{array}{llll}\text { C } & 4.996546 & 0.878824 & -2.891009\end{array}$

H $\quad 3.522484 \quad-0.674687 \quad-2.734344$

$\begin{array}{llll}\text { C } & 4.821703 & 2.678412 & -1.291732\end{array}$

H $\quad 3.205069 \quad 2.515070 \quad 0.123505$

$\begin{array}{llll}\text { C } & 5.473232 & 2.086409 & -2.375164\end{array}$

H $\quad 5.503540 \quad 0.405962 \quad-3.727765$

$\mathrm{H} \quad 5.191710 \quad 3.611204 \quad-0.874563$

$\begin{array}{llll}\mathrm{H} & 6.350292 & 2.557469 & -2.810317\end{array}$

$\mathrm{H} \quad 1.817242 \quad 0.694424 \quad 0.355880$

$\begin{array}{llll}\text { C } & 3.268926 & -1.840343 & 0.065243\end{array}$

$\begin{array}{llll}\text { C } & 4.015943 & -1.186912 & 1.056115\end{array}$

$\begin{array}{llll}\text { C } & 3.719423 & -3.078076 & -0.408679\end{array}$

$\begin{array}{lllll}\text { C } & 5.184854 & -1.759826 & 1.555406\end{array}$

$\begin{array}{llll}\mathrm{H} & 3.689092 & -0.225167 & 1.439971\end{array}$

$\begin{array}{llll}\text { C } & 4.889169 & -3.655001 & 0.088926\end{array}$

H $\quad 3.149990 \quad-3.594901-1.179205$

$\begin{array}{llll}\text { C } & 5.625918 & -2.994938 & 1.073612\end{array}$

$\mathrm{H} \quad 5.753454 \quad-1.238971 \quad 2.320861$

H $\quad 5.224803 \quad-4.614651 \quad-0.294649$

H $\quad \begin{array}{llll}6.538763 & -3.438068 & 1.462009\end{array}$

H $\quad 1.781399 \quad-1.719850 \quad-1.450151$

$\begin{array}{lllll}\text { C } & 0.979366 & -1.751992 & 1.811571\end{array}$

$\begin{array}{lllll}\mathrm{H} & 0.004204 & -1.930222 & 2.270327\end{array}$

H $\quad \begin{array}{llll}1.365192 & -0.811043 & 2.197952\end{array}$

$\begin{array}{lllll}\mathrm{H} & 1.670345 & -2.559258 & 2.077612\end{array}$

$\begin{array}{llll}\mathrm{H} & 0.446358 & -2.561445 & 0.034635\end{array}$

$\mathrm{H} \quad 0.488308 \quad 1.600780 \quad-1.123960$

$\begin{array}{llll}\text { C } & 0.710084 & 0.515711 & -2.829504\end{array}$

$\mathrm{H} \quad \begin{array}{llll}1.509776 & 1.098843 & -3.301873\end{array}$

$\begin{array}{lllll}\mathrm{H} & -0.256791 & 0.863145 & -3.197324\end{array}$

H $\quad 0.827367 \quad-0.532732 \quad-3.122491$

\section{TS4-7}

$\begin{array}{llll}\text { C } & -0.502356 & 2.488637 & -0.869935\end{array}$

$\begin{array}{llll}\text { C } & 0.408357 & 2.301780 & -1.943812\end{array}$

$\begin{array}{llll}\text { C } & -0.238652 & 3.493993 & 0.065961\end{array}$

$\begin{array}{llll}\text { C } & 1.560982 & 3.069430 & -2.086049\end{array}$

$\begin{array}{lllll}\text { C } & 0.920014 & 4.273453 & -0.069403\end{array}$

$\mathrm{H} \quad \begin{array}{llll}0.946330 & 3.670834 & 0.866779\end{array}$

C $\quad 1.808852 \quad 4.067108 \quad-1.129694$

$\mathrm{H} \quad 2.236898 \quad 2.913968 \quad-2.922292$

$\begin{array}{llll}\mathrm{H} & 1.121976 & 5.056622 & 0.656095\end{array}$

H $\quad 2.698722 \quad 4.684280 \quad-1.220305$

$\begin{array}{llll}\text { C } & -1.335611 & 0.829480 & -2.303520\end{array}$

$\begin{array}{llll}\mathrm{O} & -2.015811 & 0.035996 & -2.950550\end{array}$

$\mathrm{N} \quad \begin{array}{llll}-0.067680 & 1.285320 & -2.759664\end{array}$

$\begin{array}{llll}\text { C } & 0.452757 & 0.967298 & -4.069260\end{array}$

$\begin{array}{llll}\mathrm{H} & 0.507031 & 1.863460 & -4.700675\end{array}$

H $\quad 1.456769 \quad 0.527130 \quad-4.002690$

$\mathrm{H} \quad-0.230376 \quad 0.246560 \quad-4.523680$

$\begin{array}{lllll}\text { C } & -2.884157 & 1.736991 & -0.396061\end{array}$

$\begin{array}{llll}\text { C } & -3.627776 & -0.601884 & -0.722735\end{array}$

$\begin{array}{llll}\text { C } & -4.198918 & -2.718715 & -1.784268\end{array}$

$\begin{array}{llll}\mathrm{O} & -3.135996 & 2.778935 & 0.212570\end{array}$

N $\quad-3.889103 \quad 0.772921 \quad-0.533042$

$\begin{array}{llll}\text { C } & -2.628732 & -1.277032 & -0.001965\end{array}$

$\begin{array}{llll}\text { C } & -3.221337 & -3.379640 & -1.036532\end{array}$

$\mathrm{H} \quad-4.810977 \quad-3.270508-2.491773$

$\begin{array}{lllll}\text { C } & -5.202123 & 1.141254 & 0.009471\end{array}$

$\begin{array}{llll}\text { C } & -2.444342 & -2.657746 & -0.129844\end{array}$

H $\quad-3.075785-4.451834-1.135705$

$\mathrm{H} \quad-5.950358 \quad 0.437757 \quad-0.359655$

$\begin{array}{llll}\mathrm{H} & -5.193494 & 1.118382 & 1.106075\end{array}$

H $\quad-5.4552352 .152225 \quad-0.311649$

$\begin{array}{llll}\mathrm{H} & -1.719009 & -3.164138 & 0.498013\end{array}$

$\begin{array}{llll}\text { C } & -1.558684 & 1.483767 & -0.997412\end{array}$

$\begin{array}{llll}\text { C } & -4.385958 & -1.347472 & -1.634907\end{array}$

$\mathrm{H} \quad-5.106675 \quad-0.818809-2.250734$

I $\quad-2.441416 \quad-0.656486 \quad 2.448654$

$\begin{array}{lllll}\mathrm{Cu} & -0.925286 & -0.206128 & 0.351377\end{array}$

$\begin{array}{lllll}\mathrm{N} & 0.830775 & 0.656672 & 1.211551\end{array}$

$\begin{array}{llll}\text { C } & 1.970379 & -0.275899 & 1.048340\end{array}$

$\begin{array}{lllll}\text { C } & 1.892947 & -0.888762 & -0.373133\end{array}$

$\begin{array}{lllll}\mathrm{N} & 0.591914 & -1.584803 & -0.517913\end{array}$

$\begin{array}{llll}\mathrm{H} & 0.582275 & -2.382868 & 0.118985\end{array}$ 


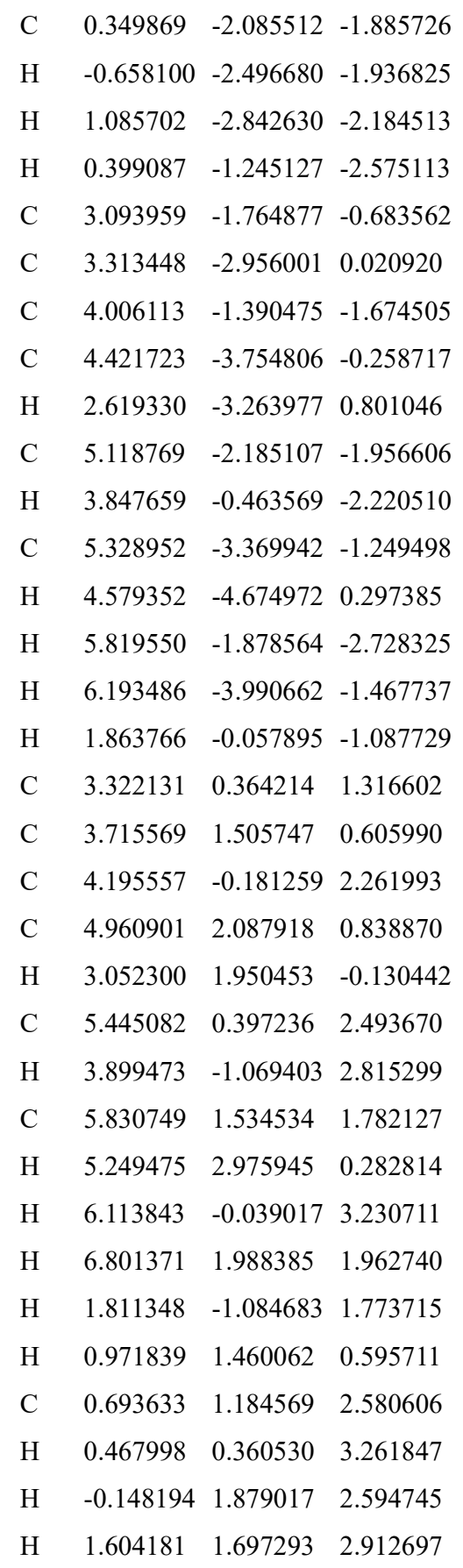

\section{TS4-8}

$\begin{array}{llll}\text { C } & 2.298982 & 2.316778 & 0.191801\end{array}$

$\begin{array}{llll}\text { C } & 1.995205 & 2.858328 & 1.465637\end{array}$

$\begin{array}{llll}\text { C } & 2.318422 & 3.162768 & -0.919868\end{array}$

$\begin{array}{llll}\text { C } & 1.672206 & 4.199897 & 1.643683\end{array}$

$\begin{array}{lllll}\text { C } & 2.008337 & 4.518320 & -0.749428\end{array}$

$\mathrm{H} \quad 2.593888 \quad 2.764082 \quad-1.888730$

$\begin{array}{llll}\text { C } & 1.678532 & 5.028864 & 0.512998\end{array}$

$\begin{array}{llll}\mathrm{H} & 1.442764 & 4.596685 & 2.628568\end{array}$

$\begin{array}{llll}\mathrm{H} & 2.028363 & 5.183378 & -1.608670\end{array}$

\begin{tabular}{|c|c|c|c|}
\hline$H$ & 1.437993 & 6.082781 & 0.624232 \\
\hline $\mathrm{C}$ & 2.556027 & 0.648620 & 1.827342 \\
\hline $\mathrm{O}$ & 2.842993 & -0.350573 & 2.488543 \\
\hline $\mathrm{N}$ & 2.108519 & 1.852046 & 2.418556 \\
\hline$C$ & 2.063662 & 2.053354 & 3.847425 \\
\hline . & 2.800138 & 2.803811 & 4.162001 \\
\hline $\mathrm{H}$ & 1.069208 & 2.386536 & 4.171808 \\
\hline $\mathrm{H}$ & 2.298537 & 1.097017 & 4.318727 \\
\hline $\mathrm{C}$ & 3.523352 & 0.231251 & -0.557101 \\
\hline $\mathrm{C}$ & 2.855633 & -2.034209 & 0.161202 \\
\hline $\mathrm{C}$ & 2.432776 & -4.057323 & 1.456954 \\
\hline $\mathrm{O}$ & 4.124118 & 0.855816 & -1.430716 \\
\hline $\mathrm{N}$ & 3.776535 & -1.132437 & -0.407147 \\
\hline$c$ & 1.477173 & -1.948044 & -0.081855 \\
\hline $\mathrm{C}$ & 1.074515 & -3.998597 & 1.139367 \\
\hline $\mathrm{H}$ & 2.816163 & -4.863558 & 2.075516 \\
\hline $\mathrm{C}$ & 4.815825 & -1.678509 & -1.289237 \\
\hline $\mathrm{C}$ & 0.601177 & -2.949863 & 0.348806 \\
\hline $\mathrm{H}$ & 0.388451 & -4.771889 & 1.473590 \\
\hline 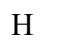 & 5.715120 & -1.064960 & -1.216635 \\
\hline $\mathrm{H}$ & 5.041573 & -2.701541 & -0.986150 \\
\hline $\mathrm{H}$ & 4.479602 & -1.674025 & -2.332713 \\
\hline $\mathrm{H}$ & -0.435584 & -2.935852 & 0.029457 \\
\hline $\mathrm{C}$ & 2.563822 & 0.886881 & 0.362712 \\
\hline $\mathrm{C}$ & 3.304254 & -3.083762 & 0.977176 \\
\hline $\mathrm{H}$ & 4.354853 & -3.103187 & 1.249440 \\
\hline 1 & 0.757626 & -1.300478 & -2.513423 \\
\hline $\mathrm{Cu}$ & 0.669822 & -0.068098 & -0.126678 \\
\hline $\mathrm{N}$ & -0.945288 & 1.264527 & -0.706339 \\
\hline $\mathrm{C}$ & -2.142737 & 0.401080 & -0.459507 \\
\hline $\mathrm{C}$ & -2.208776 & 0.074238 & 1.047736 \\
\hline $\mathrm{N}$ & -0.890378 & -0.481241 & 1.460915 \\
\hline $\mathrm{H}$ & -0.941954 & -1.495598 & 1.397080 \\
\hline $\mathrm{C}$ & -0.512771 & -0.153185 & 2.846362 \\
\hline $\mathrm{H}$ & -0.377600 & 0.927550 & 2.925122 \\
\hline 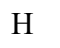 & 0.446180 & -0.628285 & 3.064595 \\
\hline $\mathrm{H}$ & -1.271336 & -0.477738 & 3.573253 \\
\hline $\mathrm{C}$ & -3.345401 & -0.859639 & 1.418946 \\
\hline $\mathrm{C}$ & -3.551668 & -2.056781 & 0.716691 \\
\hline $\mathrm{C}$ & -4.164652 & -0.575848 & 2.517625 \\
\hline $\mathrm{C}$ & -4.554078 & -2.944978 & 1.104615 \\
\hline $\mathrm{H}$ & -2.934441 & -2.296598 & -0.146037 \\
\hline $\mathrm{C}$ & -5.169421 & -1.463166 & 2.909003 \\
\hline $\mathrm{H}$ & -4.017075 & 0.350222 & 3.068725 \\
\hline
\end{tabular}




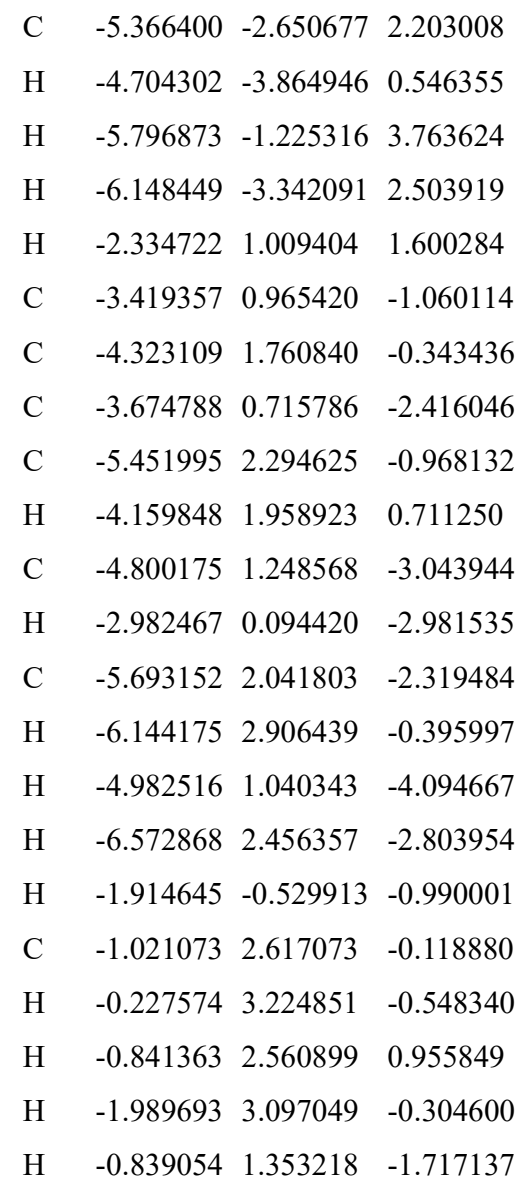

\section{TS5-1}

$\begin{array}{llll}\mathrm{C} & -1.110985 & 2.312753 & -0.779764 \\ \mathrm{C} & -1.493679 & 3.306289 & 0.152299 \\ \mathrm{C} & 0.020721 & 2.526809 & -1.563866 \\ \mathrm{C} & -0.733075 & 4.448745 & 0.372399 \\ \mathrm{C} & 0.798378 & 3.672082 & -1.355903 \\ \mathrm{H} & 0.267230 & 1.827905 & -2.348302 \\ \mathrm{C} & 0.433932 & 4.612235 & -0.385751 \\ \mathrm{H} & -1.042684 & 5.198728 & 1.092853 \\ \mathrm{H} & 1.678102 & 3.840914 & -1.970183 \\ \mathrm{H} & 1.046854 & 5.496029 & -0.233127 \\ \mathrm{C} & -3.293979 & 1.905068 & 0.009492 \\ \mathrm{O} & -4.478338 & 1.637957 & 0.003019 \\ \mathrm{~N} & -2.734884 & 2.985029 & 0.698678 \\ \mathrm{C} & -3.543412 & 3.902190 & 1.478060 \\ \mathrm{H} & -3.703983 & 4.843592 & 0.937241 \\ \mathrm{H} & -3.054489 & 4.118590 & 2.433557 \\ \mathrm{H} & -4.506813 & 3.423507 & 1.657959 \\ \mathrm{C} & -2.472809 & 0.527361 & -2.045913 \\ \mathrm{C} & -3.601102 & -1.008400 & -0.583165 \\ \mathrm{C} & -5.124832 & -1.969466 & 1.022649\end{array}$
O $\quad-2.063120 \quad 0.910887 \quad-3.138125$
N $\quad-3.254962-0.597309 \quad-1.883802$
$\begin{array}{llll}\text { C } & -2.774357 & -0.630124 & 0.479092\end{array}$
$\begin{array}{lllll}\text { C } & -4.347515 & -1.475171 & 2.074461\end{array}$
H $\quad-6.036528 \quad-2.5198891 .236128$
$\begin{array}{llll}\text { C } & -3.762426 & -1.295711 & -3.056725\end{array}$
$\begin{array}{llll}\text { C } & -3.172509 & -0.770581 & 1.800560\end{array}$
$\mathrm{H} \quad-4.628832 \quad-1.672571 \quad 3.104863$
H $\quad-3.636099-2.374156-2.924282$
$\mathrm{H} \quad-3.192484-0.954438 \quad-3.920481$
$\mathrm{H} \quad-4.823873-1.073181 \quad-3.219189$
$\begin{array}{llll}\mathrm{H} & -2.530894 & -0.436515 & 2.608927\end{array}$
$\begin{array}{llll}\text { C } & -2.132872 & 1.245843 & -0.764062\end{array}$
$\begin{array}{llll}\text { C } & -4.768607 & -1.729085 & -0.303141\end{array}$
$\mathrm{H} \quad-5.419653 \quad-2.049580-1.109875$
I $\quad-0.327144 \quad-3.026554 \quad 1.174668$
$\begin{array}{lllll}\mathrm{Cu} & -0.887171 & -0.344815 & 0.129588\end{array}$
$\mathrm{N} \quad 0.779275 \quad-0.803258 \quad-1.214486$
$\begin{array}{llll}\text { C } & 2.004368 & -0.085586 & -0.779540\end{array}$
$\begin{array}{lllll}C & 2.089903 & -0.159661 & 0.770764\end{array}$
$\begin{array}{lllll}\mathrm{N} & 0.817706 & 0.301100 & 1.416938\end{array}$
$\begin{array}{llll}\text { C } & 3.338247 & 0.501461 & 1.328238\end{array}$
$\begin{array}{llll}\text { C } & 3.773207 & 1.764234 & 0.900124\end{array}$
$\begin{array}{llll}\text { C } & 4.067757 & -0.152907 & 2.328101\end{array}$
$\begin{array}{llll}\text { C } & 4.907810 & 2.353865 & 1.456726\end{array}$
$\begin{array}{llll}\mathrm{H} & 3.221335 & 2.296852 & 0.130740\end{array}$
$\begin{array}{llll}\text { C } & 5.203875 & 0.433638 & 2.888542\end{array}$
$\begin{array}{llll}\mathrm{H} & 3.744561 & -1.135337 & 2.665019\end{array}$
$\begin{array}{llll}\text { C } & 5.627971 & 1.690094 & 2.452876\end{array}$
$\begin{array}{llll}\mathrm{H} & 5.231282 & 3.332137 & 1.110748\end{array}$
H $\quad 5.759196 \quad-0.093583 \quad 3.659596$
$\begin{array}{llll}\mathrm{H} & 6.514104 & 2.148267 & 2.883741\end{array}$
$\begin{array}{llll}\mathrm{H} & 2.137535 & -1.224211 & 1.015609\end{array}$
$\begin{array}{llll}\text { C } & 3.270775 & -0.642708 & -1.410983\end{array}$
$\begin{array}{llll}\text { C } & 3.625294 & -1.987119 & -1.228115\end{array}$
$\begin{array}{llll}\text { C } & 4.095141 & 0.171690 & -2.193132\end{array}$
$\begin{array}{llll}\text { C } & 4.785203 & -2.498809 & -1.809146\end{array}$
H $\quad 2.992275 \quad-2.641398 \quad-0.631876$
$\begin{array}{llll}\text { C } & 5.258241 & -0.336516 & -2.774797\end{array}$
H $3.826922 \quad 1.215127 \quad-2.342593$
$\begin{array}{llll}\text { C } & 5.606757 & -1.674216 & -2.582735\end{array}$
H $\quad 5.047633 \quad-3.542357 \quad-1.657728$
$\mathrm{H} \quad 5.888916 \quad 0.311342 \quad-3.377785$
$\begin{array}{llll}\mathrm{H} & 6.510639 & -2.073675 & -3.034553\end{array}$ 


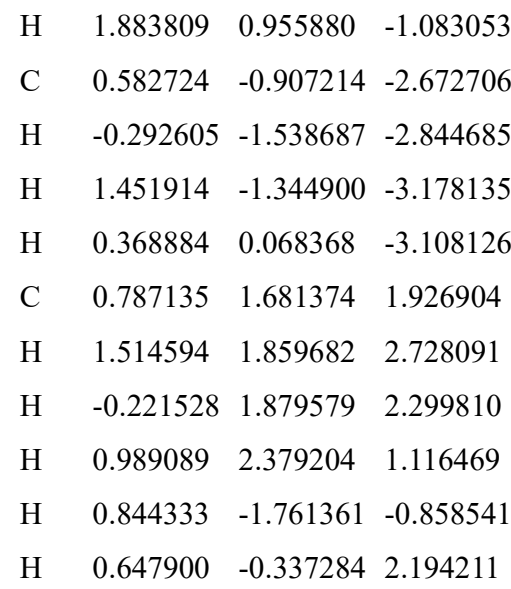

\section{TS5-2}

$\begin{array}{llll}\text { C } & -1.126489 & 2.439793 & -0.216195\end{array}$

$\begin{array}{llll}\text { C } & -1.635047 & 3.127541 & 0.913078\end{array}$

$\begin{array}{llll}\text { C } & 0.021932 & 2.933549 & -0.836965\end{array}$

$\begin{array}{llll}\text { C } & -0.953864 & 4.183337 & 1.507052\end{array}$

$\begin{array}{llll}\text { C } & 0.710087 & 4.009075 & -0.261649\end{array}$

$\begin{array}{llll}\mathrm{H} & 0.337807 & 2.529808 & -1.789170\end{array}$

$\begin{array}{llll}\text { C } & 0.244107 & 4.605039 & 0.914335\end{array}$

H $\quad-1.356500 \quad 4.691092 \quad 2.377431$

$\begin{array}{llll}\mathrm{H} & 1.603491 & 4.392265 & -0.745944\end{array}$

$\begin{array}{llll}\mathrm{H} & 0.793056 & 5.431951 & 1.355723\end{array}$

$\begin{array}{lllll}\text { C } & -3.367724 & 1.844238 & 0.163398\end{array}$

$\begin{array}{lllll}\text { O } & -4.534451 & 1.630239 & -0.091645\end{array}$

$\begin{array}{lllll}\mathrm{N} & -2.918397 & 2.663130 & 1.206182\end{array}$

$\begin{array}{llll}\text { C } & -3.840310 & 3.343101 & 2.095393\end{array}$

$\begin{array}{llll}\mathrm{H} & -3.956430 & 4.397897 & 1.814471\end{array}$

$\begin{array}{llll}\mathrm{H} & -3.479205 & 3.286178 & 3.127315\end{array}$

$\mathrm{H} \quad-4.806524 \quad 2.843233 \quad 2.016491$

$\begin{array}{llll}\text { C } & -2.189116 & 0.891547 & -1.991372\end{array}$

$\begin{array}{lllll}\text { C } & -3.464297 & -0.859421 & -0.997436\end{array}$

$\begin{array}{llll}\text { C } & -5.130408 & -2.086520 & 0.235304\end{array}$

$\begin{array}{lllll}\text { O } & -1.564846 & 1.418839 & -2.914472\end{array}$

$\begin{array}{lllll}\text { N } & -2.972790 & -0.227410 & -2.153406\end{array}$

$\begin{array}{llll}\text { C } & -2.778536 & -0.636520 & 0.201986\end{array}$

$\begin{array}{lllll}\text { C } & -4.505103 & -1.743197 & 1.438836\end{array}$

H $\quad-6.038844 \quad-2.681546 \quad 0.248648$

$\begin{array}{llll}\text { C } & -3.251725 & -0.745067 & -3.485177\end{array}$

$\begin{array}{lllll}\text { C } & -3.335823 & -0.980069 & 1.427614\end{array}$

$\mathrm{H} \quad-4.901850 \quad-2.103534 \quad 2.383582$

H $\quad-3.064832-1.822961-3.508010$

H $\quad-2.590806 \quad-0.236119-4.186454$

$\begin{array}{lllll}\mathrm{H} & -4.293320 & -0.553764 & -3.768277\end{array}$
$\begin{array}{llll}\mathrm{H} & -2.813458 & -0.758330 & 2.351841\end{array}$

$\begin{array}{lllll}\text { C } & -2.096614 & 1.382209 & -0.573339\end{array}$

$\begin{array}{llll}\text { C } & -4.627506 & -1.635366 & -0.984099\end{array}$

H $\quad-5.162249 \quad-1.836022-1.906905$

$\begin{array}{lllll}\text { I } & -0.452822 & -3.123396 & 0.834447\end{array}$

$\begin{array}{lllll}\mathrm{Cu} & -0.833178 & -0.394674 & 0.157162\end{array}$

$\begin{array}{lllll}\mathrm{N} & 0.727890 & -0.191639 & -1.400659\end{array}$

$\begin{array}{lllll}\text { C } & 1.984378 & 0.270685 & -0.730563\end{array}$

$\begin{array}{llll}\text { C } & 2.094682 & -0.410977 & 0.652059\end{array}$

$\begin{array}{lllll}\mathrm{N} & 0.900652 & -0.029735 & 1.470447\end{array}$

$\begin{array}{llll}\text { C } & 3.403589 & -0.180455 & 1.385593\end{array}$

$\begin{array}{llll}\text { C } & 4.100072 & 1.034529 & 1.330566\end{array}$

$\begin{array}{llll}\text { C } & 3.904193 & -1.204858 & 2.200026\end{array}$

$\begin{array}{llll}\text { C } & 5.271297 & 1.217582 & 2.066231\end{array}$

$\begin{array}{llll}\mathrm{H} & 3.735279 & 1.841291 & 0.701524\end{array}$

$\begin{array}{llll}\text { C } & 5.074720 & -1.025130 & 2.938471\end{array}$

$\begin{array}{llll}\mathrm{H} & 3.369408 & -2.151224 & 2.250836\end{array}$

$\begin{array}{llll}\text { C } & 5.762812 & 0.188263 & 2.872363\end{array}$

$\begin{array}{llll}\mathrm{H} & 5.802371 & 2.163961 & 2.006664\end{array}$

$\begin{array}{llll}\mathrm{H} & 5.450656 & -1.833349 & 3.560123\end{array}$

$\begin{array}{lllll}\mathrm{H} & 6.677003 & 0.330301 & 3.442411\end{array}$

$\begin{array}{lllll}\mathrm{H} & 1.971412 & -1.486956 & 0.501682\end{array}$

$\begin{array}{lllll}\text { C } & 3.209347 & 0.139017 & -1.618168\end{array}$

$\begin{array}{llll}\text { C } & 3.991996 & -1.022490 & -1.656779\end{array}$

$\begin{array}{llll}\text { C } & 3.537550 & 1.197125 & -2.475226\end{array}$

$\begin{array}{llll}\text { C } & 5.077281 & -1.119163 & -2.527875\end{array}$

H $\quad 3.761345 \quad-1.853493 \quad-0.997391$

$\begin{array}{llll}\text { C } & 4.621508 & 1.104959 & -3.349238\end{array}$

$\begin{array}{llll}\mathrm{H} & 2.937556 & 2.105456 & -2.455040\end{array}$

$\begin{array}{lllll}\text { C } & 5.395857 & -0.056538 & -3.376733\end{array}$

H $\quad 5.676242 \quad-2.025674 \quad-2.541299$

$\mathrm{H} \quad 4.862246 \quad 1.938626 \quad-4.003520$

H $\quad 6.242972-0.132664 \quad-4.052952$

$\begin{array}{lllll}\mathrm{H} & 1.822811 & 1.334548 & -0.547479\end{array}$

$\begin{array}{lllll}\text { C } & 0.831769 & -1.424537 & -2.209991\end{array}$

H $\quad-0.171033 \quad-1.666433 \quad-2.570273$

$\begin{array}{llll}\mathrm{H} & 1.157351 & -2.253392 & -1.581715\end{array}$

H $\quad 1.515054 \quad-1.312179 \quad-3.059633$

$\begin{array}{llll}\mathrm{H} & 0.414904 & 0.543143 & -2.032530\end{array}$

$\begin{array}{lllll}\mathrm{H} & 0.743659 & -0.795491 & 2.127057\end{array}$

$\begin{array}{llll}\text { C } & 0.992653 & 1.231582 & 2.224254\end{array}$

$\begin{array}{llll}\mathrm{H} & 0.029526 & 1.406311 & 2.710456\end{array}$

$\begin{array}{lllll}\mathrm{H} & 1.175182 & 2.062194 & 1.542826\end{array}$

$\begin{array}{lllll}\mathrm{H} & 1.786750 & 1.220167 & 2.980556\end{array}$ 


\section{TS5-3}

$\begin{array}{llll}\text { C } & -1.242090 & 2.232333 & -0.839251\end{array}$

$\begin{array}{llll}\text { C } & -1.635239 & 3.167551 & 0.146403\end{array}$

$\begin{array}{lllll}\text { C } & -0.117369 & 2.491978 & -1.618793\end{array}$

$\begin{array}{llll}\text { C } & -0.862877 & 4.283577 & 0.449123\end{array}$

C $\quad 0.650188 \quad 3.632840 \quad-1.349764$

$\mathrm{H} \quad 0.135964 \quad 1.826685 \quad-2.431631$

$\begin{array}{lllll}\text { C } & 0.297208 & 4.499712 & -0.309294\end{array}$

$\mathrm{H} \quad-1.166218 \quad 4.984996 \quad 1.219550$

$\mathrm{H} \quad 1.523875 \quad 3.846267 \quad-1.958460$

H $\quad 0.912726 \quad 5.369774 \quad-0.099795$

$\begin{array}{llll}\text { C } & -3.432754 & 1.775633 & -0.114667\end{array}$

$\begin{array}{lllll}\text { O } & -4.613346 & 1.502175 & -0.173439\end{array}$

$\begin{array}{llll}\mathrm{N} & -2.890792 & 2.819014 & 0.645851\end{array}$

$\begin{array}{llll}\text { C } & -3.715601 & 3.690919 & 1.459198\end{array}$

$\mathrm{H} \quad-3.845547 \quad 4.670031 \quad 0.980910$

$\mathrm{H} \quad-3.259891 \quad 3.832572 \quad 2.444799$

H $\quad-4.690270 \quad 3.213945 \quad 1.570968$

$\begin{array}{llll}\text { C } & -2.491300 & 0.416931 & -2.155022\end{array}$

C $\quad-3.526363-1.182968-0.721485$

$\begin{array}{llll}\text { C } & -5.019742 & -2.223966 & 0.859903\end{array}$

$\begin{array}{llll}\text { O } & -2.091453 & 0.845662 & -3.233326\end{array}$

$\begin{array}{llll}\mathrm{N} & -3.168858 & -0.773423 & -2.016102\end{array}$

$\begin{array}{llll}\text { C } & -2.786151 & -0.676635 & 0.350775\end{array}$

$\begin{array}{llll}\text { C } & -4.347244 & -1.601951 & 1.917360\end{array}$

$\mathrm{H} \quad-5.879538 \quad-2.855876 \quad 1.062455$

$\begin{array}{llll}\text { C } & -3.582014 & -1.516760 & -3.197199\end{array}$

$\begin{array}{llll}\text { C } & -3.241232 & -0.790707 & 1.660418\end{array}$

$\mathrm{H} \quad-4.656504 \quad-1.778378 \quad 2.943404$

H $\quad-3.366861 \quad-2.580020 \quad-3.055912$

$\mathrm{H} \quad-3.020519-1.129525-4.047165$

H $\quad-4.653968$-1.387612 -3.388776

H $\quad-2.682164 \quad-0.3501192 .478509$

$\begin{array}{llll}\text { C } & -2.241330 & 1.143638 & -0.857030\end{array}$

$\begin{array}{lllll}\text { C } & -4.628669 & -2.006303 & -0.459393\end{array}$

H $\quad-5.205103 \quad-2.425066-1.277758$

I $\quad \begin{array}{llll}0.171818 & -2.702438 & 1.544551\end{array}$

$\begin{array}{lllll}\mathrm{Cu} & -0.875923 & -0.287217 & 0.206029\end{array}$

$\begin{array}{lllll}\mathrm{N} & 0.765413 & -0.860027 & -1.251945\end{array}$

$\begin{array}{llll}\text { C } & 2.017257 & -0.147596 & -0.898261\end{array}$

$\begin{array}{llll}\mathrm{C} & 2.047683 & 0.148066 & 0.629734\end{array}$

$\begin{array}{llll}\mathrm{N} & 0.791447 & 0.825773 & 1.039876\end{array}$

$\begin{array}{llll}\mathrm{H} & 0.773066 & 1.750907 & 0.614136\end{array}$

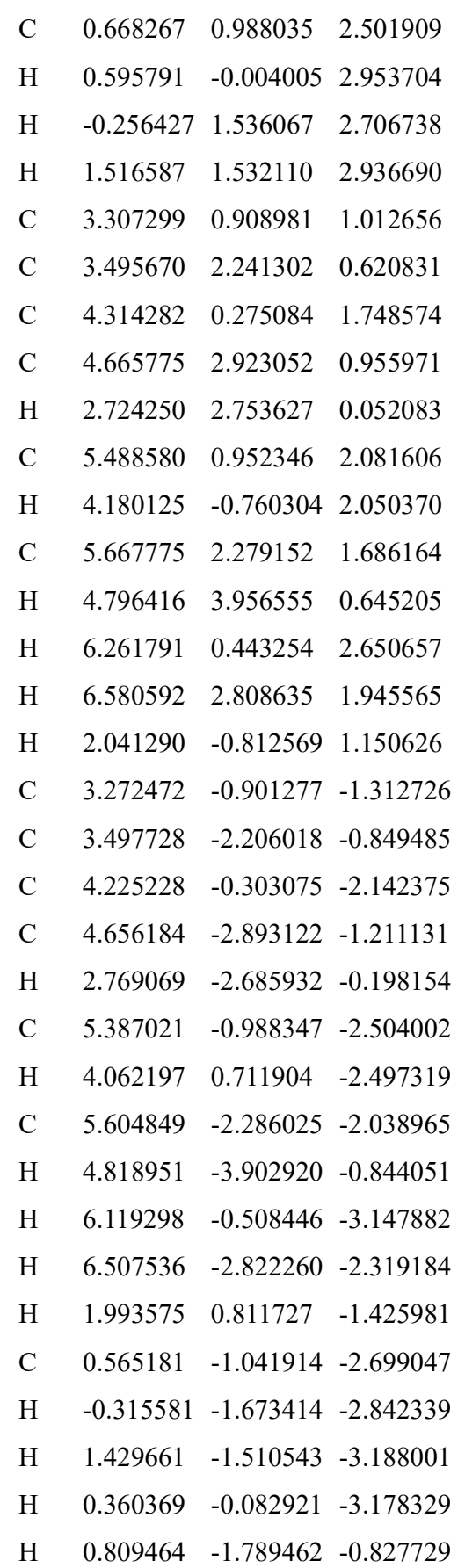

\section{TS5-4}

$\begin{array}{llll}\text { C } & -1.385894 & 2.241814 & -0.825745\end{array}$

$\begin{array}{lllll}\text { C } & -2.073081 & 3.116420 & 0.048784\end{array}$

$\begin{array}{lllll}\text { C } & -0.198407 & 2.671126 & -1.419670\end{array}$

$\begin{array}{llll}\text { C } & -1.532988 & 4.334121 & 0.446693\end{array}$

$\begin{array}{lllll}\text { C } & 0.340908 & 3.912457 & -1.053083\end{array}$

$\begin{array}{llll}\mathrm{H} & 0.273603 & 2.061126 & -2.178080\end{array}$

$\begin{array}{lllll}\text { C } & -0.302051 & 4.716093 & -0.105738\end{array}$

$\begin{array}{llll}\mathrm{H} & -2.064639 & 4.986396 & 1.131922\end{array}$

H $\quad 1.259079 \quad 4.256056 \quad-1.520411$ 


$$
\begin{aligned}
& \mathrm{H} \quad 0.136396 \quad 5.667254 \quad 0.181675 \\
& \text { C } \quad-3.590916 \quad 1.494261 \quad-0.499382 \\
& \begin{array}{lllll}
\mathrm{O} & -4.693435 & 1.062049 & -0.765474
\end{array} \\
& \begin{array}{llll}
\mathrm{N} & -3.341428 & 2.601232 & 0.319463
\end{array} \\
& \begin{array}{llll}
\text { C } & -4.409138 & 3.351219 & 0.951030
\end{array} \\
& \mathrm{H} \quad-4.580645 \quad 4.304379 \quad 0.434760 \\
& \begin{array}{llll}
\mathrm{H} & -4.162320 & 3.551731 & 1.998829
\end{array} \\
& \text { H } \quad-5.3148592 .745675 \quad 0.896995 \\
& \begin{array}{llll}
\text { C } & -2.092859 & 0.227722 & -2.256396
\end{array} \\
& \begin{array}{lllll}
\text { C } & -3.196639 & -1.430034 & -0.962553
\end{array} \\
& \begin{array}{llll}
\text { C } & -4.815820 & -2.580082 & 0.400127
\end{array} \\
& \begin{array}{lllll}
\text { O } & -1.469831 & 0.633518 & -3.239361
\end{array} \\
& \mathrm{~N} \quad-2.679074 \quad-1.013333-2.199537 \\
& \begin{array}{llll}
\text { C } & -2.694152 & -0.807631 & 0.186013
\end{array} \\
& \begin{array}{llll}
\text { C } & -4.401943 & -1.844984 & 1.517057
\end{array} \\
& \begin{array}{llll}
\mathrm{H} & -5.625782 & -3.297569 & 0.493237
\end{array} \\
& \begin{array}{lllll}
\text { C } & -2.775182 & -1.839341 & -3.393974
\end{array} \\
& \text { C } \quad-3.361010 \quad-0.923516 \quad 1.404138 \\
& \begin{array}{llll}
\mathrm{H} & -4.860638 & -2.017412 & 2.486309
\end{array} \\
& \mathrm{H} \quad-2.456101 \quad-2.860564 \quad-3.164789 \\
& \mathrm{H} \quad-2.123630-1.406510-4.152688 \\
& \mathrm{H} \quad-3.803998 \quad-1.860447 \quad-3.771644 \\
& \text { H } \quad-3.002013 \quad-0.394346 \quad 2.279373 \\
& \text { C } \quad-2.209671 \quad 1.025823 \quad-0.986891 \\
& \text { C } \quad-4.232423-2.364694-0.845904 \\
& \text { H } \quad-4.615829 \quad-2.869012 \quad-1.726973 \\
& \text { I } \quad-0.157755 \quad-2.287201 \quad 2.133497 \\
& \begin{array}{lllll}
\mathrm{Cu} & -0.807830 & -0.252907 & 0.364818
\end{array} \\
& \begin{array}{lllll}
\mathrm{N} & 0.689018 & -0.722021 & -1.311272
\end{array} \\
& \begin{array}{llll}
\text { C } & 2.002769 & -0.104316 & -0.941661
\end{array} \\
& \begin{array}{llll}
\text { C } & 2.080229 & 0.220202 & 0.578235
\end{array} \\
& \begin{array}{llll}
\mathrm{N} & 0.847115 & 0.949377 & 0.972982
\end{array} \\
& \mathrm{H} \quad 0.793460 \quad 1.803360 \quad 0.418351 \\
& \begin{array}{llll}
\text { C } & 0.806291 & 1.338926 & 2.397732
\end{array} \\
& \mathrm{H} \quad 0.810461 \quad 0.434835 \quad 3.008681 \\
& \begin{array}{llll}
\mathrm{H} & -0.130383 & 1.876253 & 2.572317
\end{array} \\
& \text { H } \quad 1.651099 \quad 1.980741 \quad 2.675730 \\
& \begin{array}{llll}
\text { C } & 3.356877 & 0.981474 & 0.899061
\end{array} \\
& \begin{array}{llll}
\text { C } & 3.565742 & 2.278679 & 0.410173
\end{array} \\
& \begin{array}{llll}
\text { C } & 4.349584 & 0.394952 & 1.691038
\end{array} \\
& \begin{array}{llll}
\text { C } & 4.743081 & 2.968698 & 0.698687
\end{array} \\
& \text { H } \quad 2.799616 \quad 2.761851 \quad-0.191885 \\
& \begin{array}{llll}
\text { C } & 5.531056 & 1.080260 & 1.979529
\end{array} \\
& \text { H } \quad 4.195275 \quad-0.608113 \quad 2.079633
\end{aligned}
$$

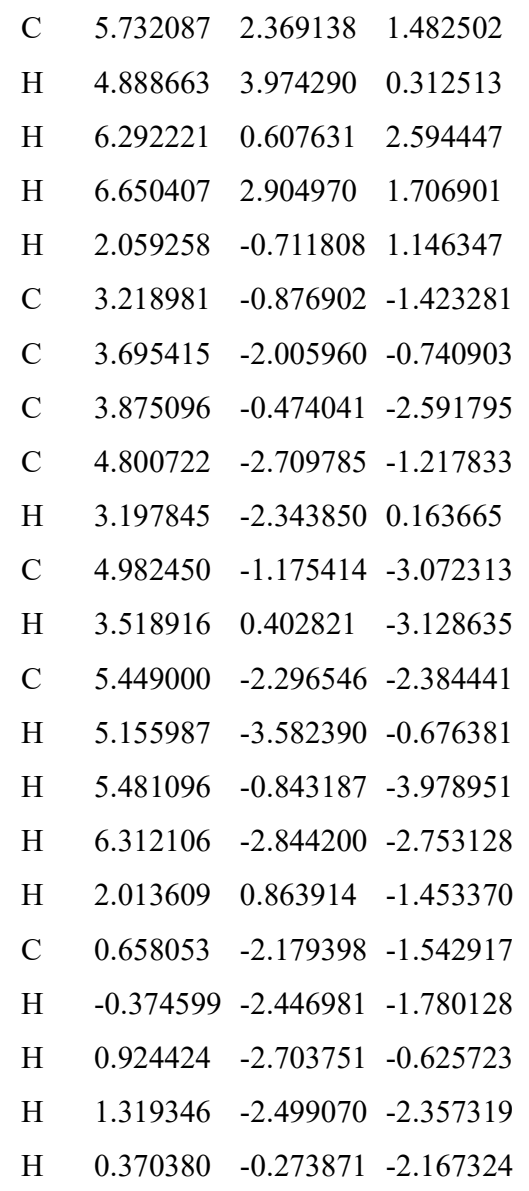

\section{TS5-5}

$\begin{array}{llll}\mathrm{N} & -0.675442 & 0.288346 & 1.430220 \\ \mathrm{C} & -1.927495 & 0.345342 & 0.619498 \\ \mathrm{C} & -1.975393 & -0.885061 & -0.326963 \\ \mathrm{~N} & -0.814825 & -0.743315 & -1.252375 \\ \mathrm{H} & -0.952939 & 0.137380 & -1.748939 \\ \mathrm{C} & -0.696713 & -1.804017 & -2.275848 \\ \mathrm{H} & -0.536553 & -2.761074 & -1.779182 \\ \mathrm{H} & 0.184749 & -1.582406 & -2.883748 \\ \mathrm{H} & -1.586379 & -1.856212 & -2.914368 \\ \mathrm{C} & -3.294090 & -1.017701 & -1.067189 \\ \mathrm{C} & -3.837542 & 0.063200 & -1.775832 \\ \mathrm{C} & -3.968312 & -2.242976 & -1.085870 \\ \mathrm{C} & -5.033252 & -0.078423 & -2.478518 \\ \mathrm{H} & -3.329123 & 1.023692 & -1.772736 \\ \mathrm{C} & -5.166632 & -2.388488 & -1.788308 \\ \mathrm{H} & -3.548774 & -3.089884 & -0.547616 \\ \mathrm{C} & -5.702890 & -1.305308 & -2.485823 \\ \mathrm{H} & -5.445690 & 0.769933 & -3.018425 \\ \mathrm{H} & -5.677578 & -3.347498 & -1.791443 \\ \mathrm{H} & -6.635364 & -1.414836 & -3.032751\end{array}$ 


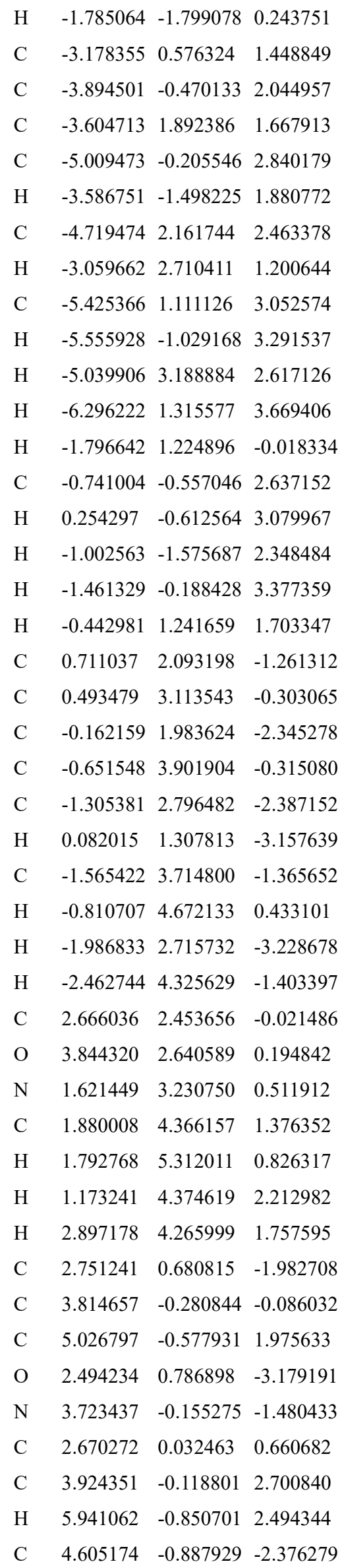

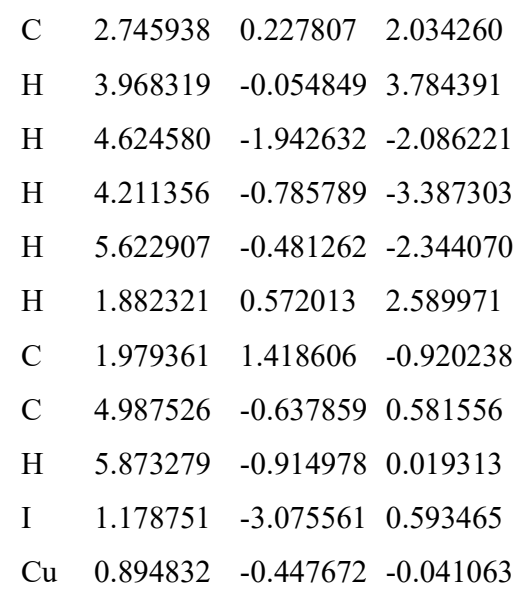

\section{TS5-6}

$\begin{array}{llll}\mathrm{N} & -0.748569 & 0.739573 & 1.135315\end{array}$

$\begin{array}{llll}\text { C } & -1.996020 & 0.592975 & 0.315232\end{array}$

$\begin{array}{llll}\text { C } & -2.044320 & -0.843624 & -0.252146\end{array}$

N $\quad-0.852083-1.041686-1.141036$

$\begin{array}{llll}\text { C } & -3.341280 & -1.249798 & -0.928429\end{array}$

$\begin{array}{llll}\text { C } & -4.148085 & -0.348610 & -1.635443\end{array}$

$\begin{array}{llll}\text { C } & -3.708440 & -2.602065 & -0.900824\end{array}$

$\begin{array}{llll}\text { C } & -5.296833 & -0.787886 & -2.294410\end{array}$

$\begin{array}{llll}\mathrm{H} & -3.891301 & 0.706344 & -1.660009\end{array}$

$\begin{array}{llll}\text { C } & -4.855470 & -3.045307 & -1.560413\end{array}$

H $\quad-3.085175 \quad-3.309971 \quad-0.358009$

$\begin{array}{llll}\text { C } & -5.654078 & -2.137537 & -2.259595\end{array}$

H $\quad-5.914962-0.074116 \quad-2.832499$

$\mathrm{H} \quad-5.126305 \quad-4.097110 \quad-1.524947$

H $\quad-6.549995 \quad-2.478608-2.771272$

$\begin{array}{llll}\mathrm{H} & -1.866660 & -1.532857 & 0.578265\end{array}$

$\begin{array}{llll}\text { C } & -3.239253 & 1.046265 & 1.061648\end{array}$

$\begin{array}{llll}\text { C } & -4.004999 & 0.178403 & 1.852014\end{array}$

$\begin{array}{llll}\text { C } & -3.601718 & 2.398554 & 1.005234\end{array}$

$\begin{array}{llll}\text { C } & -5.108441 & 0.651310 & 2.562826\end{array}$

$\begin{array}{llll}\mathrm{H} & -3.748085 & -0.875157 & 1.905328\end{array}$

$\begin{array}{llll}\text { C } & -4.704991 & 2.875223 & 1.714744\end{array}$

$\begin{array}{llll}\mathrm{H} & -3.011767 & 3.079819 & 0.396029\end{array}$

$\begin{array}{llll}\text { C } & -5.462884 & 2.000799 & 2.496180\end{array}$

$\begin{array}{llll}\mathrm{H} & -5.693898 & -0.036865 & 3.166458\end{array}$

H $\quad-4.974818 \quad 3.926395 \quad 1.653555$

$\begin{array}{llll}\mathrm{H} & -6.324970 & 2.366850 & 3.047170\end{array}$

$\begin{array}{lllll}\mathrm{H} & -1.842058 & 1.272424 & -0.525803\end{array}$

$\begin{array}{lllll}\text { C } & -0.830974 & 0.280491 & 2.535929\end{array}$

$\begin{array}{llll}\mathrm{H} & 0.132642 & 0.482180 & 3.010465\end{array}$

$\begin{array}{lllll}\mathrm{H} & -0.980340 & -0.800465 & 2.558508\end{array}$ 
H $\quad-1.625546 \quad 0.776650 \quad 3.105587$

$\begin{array}{llll}\mathrm{H} & -0.513501 & 1.730207 & 1.137344\end{array}$

$\begin{array}{llll}\text { C } & 0.980508 & 2.226209 & -1.126907\end{array}$

$\begin{array}{llll}\text { C } & 1.121747 & 3.231253 & -0.135911\end{array}$

$\begin{array}{llll}\text { C } & -0.034228 & 2.347976 & -2.074051\end{array}$

$\begin{array}{llll}\text { C } & 0.189348 & 4.251621 & 0.015438\end{array}$

$\begin{array}{llll}\text { C } & -0.960008 & 3.393038 & -1.956825\end{array}$

$\mathrm{H} \quad-0.060845 \quad 1.664901 \quad-2.908950$

C $\quad-0.868727 \quad 4.310866 \quad-0.905911$

$\begin{array}{llll}\mathrm{H} & 0.302718 & 5.006724 & 0.786609\end{array}$

H $\quad \begin{array}{llll}-1.750009 & 3.493836 & -2.695157\end{array}$

H $\quad-1.600724 \quad 5.109186 \quad-0.820033$

$\begin{array}{llll}\text { C } & 3.101985 & 2.093437 & -0.137052\end{array}$

$\begin{array}{lllll}\text { O } & 4.310803 & 2.012098 & -0.074618\end{array}$

$\begin{array}{llll}\mathrm{N} & 2.335524 & 3.062667 & 0.529270\end{array}$

$\begin{array}{llll}\text { C } & 2.943130 & 4.070882 & 1.375768\end{array}$

$\begin{array}{llll}\mathrm{H} & 3.008617 & 5.036484 & 0.857949\end{array}$

$\begin{array}{llll}\mathrm{H} & 2.358221 & 4.197050 & 2.292882\end{array}$

H $\quad 3.948470 \quad 3.729799 \quad 1.626389$

$\begin{array}{llll}\text { C } & 2.565832 & 0.433648 & -2.115583\end{array}$

$\begin{array}{llll}\text { C } & 3.706575 & -0.775187 & -0.393293\end{array}$

$\begin{array}{llll}\text { C } & 5.150486 & -1.370320 & 1.444832\end{array}$

$\begin{array}{llll}\text { O } & 2.161702 & 0.600897 & -3.265794\end{array}$

N $\quad 3.442584 \quad-0.568994 \quad-1.758583$

$\begin{array}{llll}\text { C } & 2.753365 & -0.342440 & 0.536544\end{array}$

$\begin{array}{llll}\text { C } & 4.242603 & -0.816413 & 2.352221\end{array}$

H $\quad 6.080888 \quad-1.799938 \quad 1.804465$

$\begin{array}{lllll}\text { C } & 4.065888 & -1.392563 & -2.784923\end{array}$

$\begin{array}{llll}\text { C } & 3.044449 & -0.265231 & 1.891096\end{array}$

H $\quad 4.444974 \quad-0.8477703 .418998$

H $\quad 3.983570 \quad-2.447767 \quad-2.508310$

$\mathrm{H} \quad 3.541433 \quad-1.210030 \quad-3.722546$

H $\quad 5.123619 \quad-1.131466 \quad-2.908812$

$\begin{array}{llll}\mathrm{H} & 2.311377 & 0.117519 & 2.592218\end{array}$

$\begin{array}{llll}\text { C } & 2.098474 & 1.281154 & -0.968052\end{array}$

$\begin{array}{llll}\text { C } & 4.898612 & -1.338139 & 0.073857\end{array}$

H $\quad 5.643730 \quad-1.698355 \quad-0.628018$

I $\quad 0.677015 \quad-3.011469 \quad 1.108896$

$\begin{array}{lllll}\mathrm{Cu} & 0.862813 & -0.362283 & 0.035455\end{array}$

H $\quad-0.597942 \quad-2.028494 \quad-1.057946$

$\begin{array}{llll}\text { C } & -1.057193 & -0.755773 & -2.574989\end{array}$

$\mathrm{H} \quad-1.676284 \quad-1.518870 \quad-3.059887$

$\mathrm{H} \quad-0.080448 \quad-0.701686 \quad-3.062914$

$\mathrm{H} \quad-1.560601 \quad 0.203055 \quad-2.697080$

\section{TS5-7}

$\begin{array}{llll}\text { N } & -0.766530 & -0.099393 & 1.453539\end{array}$

$\begin{array}{lllll}\text { C } & -2.042327 & 0.252768 & 0.781881\end{array}$

$\begin{array}{llll}\text { C } & -2.070965 & -0.274242 & -0.678060\end{array}$

$\begin{array}{llll}\mathrm{N} & -0.833253 & 0.160235 & -1.371195\end{array}$

$\begin{array}{lllll}\mathrm{H} & -0.842912 & 1.179722 & -1.393466\end{array}$

$\begin{array}{lllll}\text { C } & -0.713108 & -0.327989 & -2.761761\end{array}$

H $\quad-0.633711 \quad-1.416356 \quad-2.738432$

$\begin{array}{lllll}\mathrm{H} & 0.210015 & 0.079360 & -3.184935\end{array}$

$\begin{array}{lllll}\mathrm{H} & -1.567844 & -0.028693 & -3.380462\end{array}$

$\begin{array}{llll}\text { C } & -3.341888 & 0.176834 & -1.380157\end{array}$

$\begin{array}{lllll}\text { C } & -3.575700 & 1.534360 & -1.642277\end{array}$

C $\quad-4.307996 \quad-0.759393-1.761373$

$\begin{array}{lllll}\text { C } & -4.750924 & 1.944955 & -2.271157\end{array}$

$\begin{array}{lllll}\mathrm{H} & -2.838380 & 2.280132 & -1.351461\end{array}$

$\begin{array}{llll}\text { C } & -5.487400 & -0.351749 & -2.387779\end{array}$

H $\quad-4.136333-1.813023-1.557451$

$\begin{array}{lllll}\text { C } & -5.711882 & 1.001690 & -2.644766\end{array}$

$\begin{array}{lllll}\mathrm{H} & -4.918095 & 3.000709 & -2.468516\end{array}$

$\mathrm{H} \quad-6.228781 \quad-1.092162 \quad-2.675699$

$\begin{array}{lllll}\mathrm{H} & -6.628258 & 1.320727 & -3.133855\end{array}$

H $\quad-2.033562 \quad-1.365821 \quad-0.655358$

$\begin{array}{lllll}\text { C } & -3.266234 & -0.230631 & 1.544714\end{array}$

$\begin{array}{lllll}\text { C } & -3.442221 & -1.600519 & 1.790664\end{array}$

$\begin{array}{lllll}\text { C } & -4.230713 & 0.670281 & 2.005047\end{array}$

$\begin{array}{lllll}\text { C } & -4.563589 & -2.055004 & 2.483934\end{array}$

$\begin{array}{lllll}\mathrm{H} & -2.704338 & -2.316829 & 1.432461\end{array}$

$\begin{array}{llll}\text { C } & -5.355441 & 0.217185 & 2.698119\end{array}$

$\begin{array}{llll}\mathrm{H} & -4.105951 & 1.732427 & 1.807867\end{array}$

$\begin{array}{llll}\text { C } & -5.523870 & -1.147018 & 2.939518\end{array}$

$\begin{array}{llll}\mathrm{H} & -4.689382 & -3.118985 & 2.665455\end{array}$

$\begin{array}{llll}\mathrm{H} & -6.098120 & 0.929492 & 3.047642\end{array}$

$\begin{array}{llll}\mathrm{H} & -6.398106 & -1.502314 & 3.478314\end{array}$

$\begin{array}{lllll}\mathrm{H} & -2.059931 & 1.349325 & 0.736839\end{array}$

$\begin{array}{llll}\text { C } & 1.272218 & 2.402538 & -0.373341\end{array}$

$\begin{array}{llll}\text { C } & 1.296421 & 3.047705 & 0.884316\end{array}$

$\begin{array}{lllll}\text { C } & 0.409762 & 2.877778 & -1.362622\end{array}$

$\begin{array}{llll}\text { C } & 0.375798 & 4.032543 & 1.224520\end{array}$

$\begin{array}{lllll}\text { C } & -0.510850 & 3.885799 & -1.040436\end{array}$

$\begin{array}{llll}\mathrm{H} & 0.493852 & 2.494586 & -2.373360\end{array}$

$\begin{array}{lllll}\text { C } & -0.551207 & 4.427437 & 0.248081\end{array}$

$\begin{array}{lllll}\mathrm{H} & 0.398340 & 4.508869 & 2.199533\end{array}$

$\begin{array}{lllll}\mathrm{H} & -1.183147 & 4.259989 & -1.806928\end{array}$ 
$\begin{array}{llll}\mathrm{H} & -1.276838 & 5.199621 & 0.486981\end{array}$

$\begin{array}{llll}\text { C } & 3.238848 & 1.840480 & 0.787220\end{array}$

$\begin{array}{llll}\mathrm{O} & 4.418053 & 1.639051 & 0.986932\end{array}$

$\begin{array}{llll}\mathrm{N} & 2.406879 & 2.611896 & 1.608765\end{array}$

$\begin{array}{llll}\text { C } & 2.891730 & 3.249327 & 2.816569\end{array}$

H $\quad 3.035035 \quad 4.326862 \quad 2.665513$

H $2.181252 \quad 3.095399 \quad 3.635824$

$\begin{array}{llll}\mathrm{H} & 3.849231 & 2.792367 & 3.070339\end{array}$

$\begin{array}{lllll}\text { C } & 2.969367 & 0.982262 & -1.694001\end{array}$

$\begin{array}{llll}\text { C } & 3.711218 & -0.858159 & -0.394849\end{array}$

$\begin{array}{llll}\text { C } & 4.703889 & -2.267388 & 1.288148\end{array}$

$\begin{array}{llll}\text { O } & 2.784884 & 1.608385 & -2.734167\end{array}$

N $\quad 3.716856 \quad-0.169980 \quad-1.616262$

$\begin{array}{lllll}\text { C } & 2.653097 & -0.589996 & 0.484758\end{array}$

$\begin{array}{llll}\text { C } & 3.713281 & -1.891438 & 2.200047\end{array}$

$\mathrm{H} \quad 5.490777 \quad-2.950258 \quad 1.594510$

C $\quad 4.464302-0.648378-2.768976$

$\begin{array}{llll}\text { C } & 2.699506 & -1.015225 & 1.808260\end{array}$

$\mathrm{H} \quad 3.707735 \quad-2.304625 \quad 3.204537$

H $\quad 4.242233 \quad-1.706228 \quad-2.938960$

$\begin{array}{llll}\mathrm{H} & 4.155745 & -0.059149 & -3.632262\end{array}$

$\mathrm{H} \quad 5.542402 \quad-0.523684 \quad-2.614001$

H $\quad 1.904866 \quad-0.757967 \quad 2.497267$

C $\quad 2.344803 \quad 1.380926 \quad-0.380414$

C $\quad 4.721063 \quad-1.740309-0.002950$

H $\quad 5.532762 \quad-1.978867 \quad-0.682238$

I $\quad 0.341539-3.082468-0.349756$

$\mathrm{Cu} \quad 0.828551 \quad-0.344431 \quad-0.193981$

$\begin{array}{llll}\text { C } & -0.593251 & 0.638262 & 2.714149\end{array}$

$\begin{array}{llll}\mathrm{H} & 0.338629 & 0.326773 & 3.191947\end{array}$

H $\quad-1.422537 \quad 0.481535 \quad 3.418229$

$\mathrm{H} \quad-0.519110 \quad 1.705725 \quad 2.490091$

$\mathrm{H} \quad-0.772650 \quad-1.102330 \quad 1.650350$

\section{TS5-8}

$\begin{array}{llll}\mathrm{N} & -0.854231 & 0.342438 & 1.309231\end{array}$

$\begin{array}{llll}\text { C } & -2.015095 & 0.491278 & 0.394625\end{array}$

$\begin{array}{llll}\text { C } & -2.057145 & -0.718773 & -0.578415\end{array}$

$\begin{array}{llll}\mathrm{N} & -0.736239 & -0.927362 & -1.260923\end{array}$

C $\quad-3.242805-0.660424-1.524475$

$\begin{array}{llll}\text { C } & -3.593550 & 0.509865 & -2.212265\end{array}$

C $\quad-3.996605-1.818234-1.750274$

C $\quad-4.669347 \quad 0.522839 \quad-3.099325$

$\mathrm{H} \quad \begin{array}{llll}-3.023254 & 1.421597 & -2.056907\end{array}$ $\begin{array}{llll}\text { C } & -5.074279 & -1.811317 & -2.637734\end{array}$

H $\quad-3.737385-2.732196-1.220856$

C $\quad-5.414353-0.639036-3.314880$

$\mathrm{H} \quad-4.926937 \quad 1.440462 \quad-3.621565$

H $\quad-5.649593 \quad-2.719648 \quad-2.795231$

H $\quad-6.254421-0.629128-4.004000$

$\begin{array}{llll}\mathrm{H} & -2.177340 & -1.603708 & 0.052103\end{array}$

$\begin{array}{llll}\text { C } & -3.328719 & 0.632389 & 1.146902\end{array}$

$\begin{array}{llll}\text { C } & -3.778563 & -0.391211 & 1.993358\end{array}$

$\begin{array}{llll}\text { C } & -4.098029 & 1.793361 & 1.022816\end{array}$

$\begin{array}{llll}\text { C } & -4.979444 & -0.256648 & 2.689743\end{array}$

$\begin{array}{llll}\mathrm{H} & -3.188648 & -1.297842 & 2.113252\end{array}$

$\begin{array}{llll}\text { C } & -5.301452 & 1.930522 & 1.717256\end{array}$

$\begin{array}{llll}\mathrm{H} & -3.751020 & 2.595121 & 0.375067\end{array}$

$\begin{array}{llll}\text { C } & -5.746064 & 0.903794 & 2.551697\end{array}$

$\begin{array}{lllll}\mathrm{H} & -5.316922 & -1.058807 & 3.340483\end{array}$

$\begin{array}{llll}\mathrm{H} & -5.888952 & 2.838256 & 1.607418\end{array}$

$\begin{array}{llll}\mathrm{H} & -6.682141 & 1.007013 & 3.093813\end{array}$

$\mathrm{H} \quad-1.831300 \quad 1.407114 \quad-0.169378$

$\begin{array}{llll}\text { C } & 1.239516 & 2.353099 & -0.370903\end{array}$

$\begin{array}{llll}\text { C } & 1.805602 & 3.183351 & 0.622541\end{array}$

$\begin{array}{llll}\text { C } & 0.056319 & 2.745266 & -0.993102\end{array}$

$\begin{array}{llll}\text { C } & 1.175806 & 4.342504 & 1.061137\end{array}$

$\begin{array}{llll}\text { C } & -0.584466 & 3.916277 & -0.571190\end{array}$

H $\quad-0.335553 \quad 2.161603 \quad-1.814862$

$\begin{array}{llll}\text { C } & -0.039334 & 4.693738 & 0.455875\end{array}$

$\begin{array}{llll}\mathrm{H} & 1.622967 & 4.970588 & 1.824688\end{array}$

$\mathrm{H} \quad-1.505338 \quad 4.228305 \quad-1.055181$

$\begin{array}{llll}\mathrm{H} & -0.549531 & 5.597393 & 0.776910\end{array}$

$\begin{array}{llll}\text { C } & 3.464560 & 1.702156 & 0.079396\end{array}$

$\begin{array}{lllll}\text { O } & 4.611532 & 1.343692 & -0.094219\end{array}$

$\begin{array}{llll}\mathrm{N} & 3.073742 & 2.710354 & 0.962745\end{array}$

$\begin{array}{llll}\text { C } & 4.031056 & 3.448614 & 1.763137\end{array}$

H $4.203642 \quad 4.449016 \quad 1.346194$

$\begin{array}{llll}\mathrm{H} & 3.667446 & 3.547980 & 2.791092\end{array}$

$\begin{array}{llll}\mathrm{H} & 4.968370 & 2.890831 & 1.756768\end{array}$

$\begin{array}{lllll}\text { C } & 2.312213 & 0.779565 & -2.069497\end{array}$

$\begin{array}{llll}\text { C } & 3.380384 & -1.119306 & -1.089241\end{array}$

$\begin{array}{lllll}\text { C } & 4.949390 & -2.513301 & 0.099311\end{array}$

O $\quad 1.867866 \quad 1.434423 \quad-3.006198$

$\mathrm{N} \quad 2.947456 \quad-0.431705 \quad-2.233354$

$\begin{array}{llll}\text { C } & 2.749848 & -0.837647 & 0.125033\end{array}$

$\begin{array}{llll}\text { C } & 4.392651 & -2.113159 & 1.318749\end{array}$

$\begin{array}{llll}\mathrm{H} & 5.794377 & -3.195647 & 0.087916\end{array}$ 


$$
\begin{array}{llll}
\mathrm{C} & 3.271329 & -0.900453 & -3.573332 \\
\mathrm{C} & 3.306078 & -1.237871 & 1.336110 \\
\mathrm{H} & 4.774031 & -2.512084 & 2.253994 \\
\mathrm{H} & 3.033726 & -1.964930 & -3.658735 \\
\mathrm{H} & 2.675596 & -0.324567 & -4.280993 \\
\mathrm{H} & 4.334215 & -0.749229 & -3.796385 \\
\mathrm{H} & 2.836807 & -0.969823 & 2.275708 \\
\mathrm{C} & 2.175919 & 1.236546 & -0.633243 \\
\mathrm{C} & 4.463301 & -2.009581 & -1.104289 \\
\mathrm{H} & 4.955410 & -2.256801 & -2.039019 \\
\mathrm{I} & 0.149369 & -2.951780 & 1.490419 \\
\mathrm{Cu} & 0.879533 & -0.323340 & 0.258928 \\
\mathrm{H} & -0.519255 & -1.918687 & -1.151636 \\
\mathrm{C} & -0.644603 & -0.603993 & -2.694814 \\
\mathrm{H} & -1.397183 & -1.121654 & -3.301514 \\
\mathrm{H} & 0.352052 & -0.890607 & -3.035515 \\
\mathrm{H} & -0.750755 & 0.468363 & -2.860524 \\
\mathrm{H} & -0.979765 & -0.525351 & 1.838908 \\
\mathrm{C} & -0.714698 & 1.446728 & 2.277140 \\
\mathrm{H} & 0.196394 & 1.281427 & 2.858720 \\
\mathrm{H} & -1.573063 & 1.508840 & 2.956816 \\
\mathrm{H} & -0.622509 & 2.391380 & 1.743659
\end{array}
$$

$$
\begin{array}{llll}
\text { Cat } \\
\mathrm{Cu} & 1.878681 & -0.000389 & 0.000026 \\
\mathrm{~N} & 0.255075 & 1.419871 & 0.005165 \\
\mathrm{C} & -0.983983 & 0.688066 & 0.360857 \\
\mathrm{C} & -0.984015 & -0.688333 & -0.360808 \\
\mathrm{~N} & 0.254934 & -1.420217 & -0.004962 \\
\mathrm{H} & 0.164776 & -1.764953 & 0.951368 \\
\mathrm{C} & 0.539135 & -2.570862 & -0.884600 \\
\mathrm{H} & 1.448242 & -3.059109 & -0.527497 \\
\mathrm{H} & -0.287768 & -3.293345 & -0.920403 \\
\mathrm{H} & 0.734202 & -2.198084 & -1.894054 \\
\mathrm{C} & -2.253129 & -1.477169 & -0.085416 \\
\mathrm{C} & -2.569459 & -1.883802 & 1.218745 \\
\mathrm{C} & -3.124453 & -1.816526 & -1.124997 \\
\mathrm{C} & -3.730488 & -2.611411 & 1.476357 \\
\mathrm{H} & -1.909887 & -1.627591 & 2.045933 \\
\mathrm{C} & -4.289261 & -2.543991 & -0.871882 \\
\mathrm{H} & -2.892801 & -1.502533 & -2.139872 \\
\mathrm{C} & -4.595087 & -2.943287 & 0.429722 \\
\mathrm{H} & -3.961958 & -2.917336 & 2.492921 \\
\mathrm{H} & -4.955279 & -2.798515 & -1.691716
\end{array}
$$

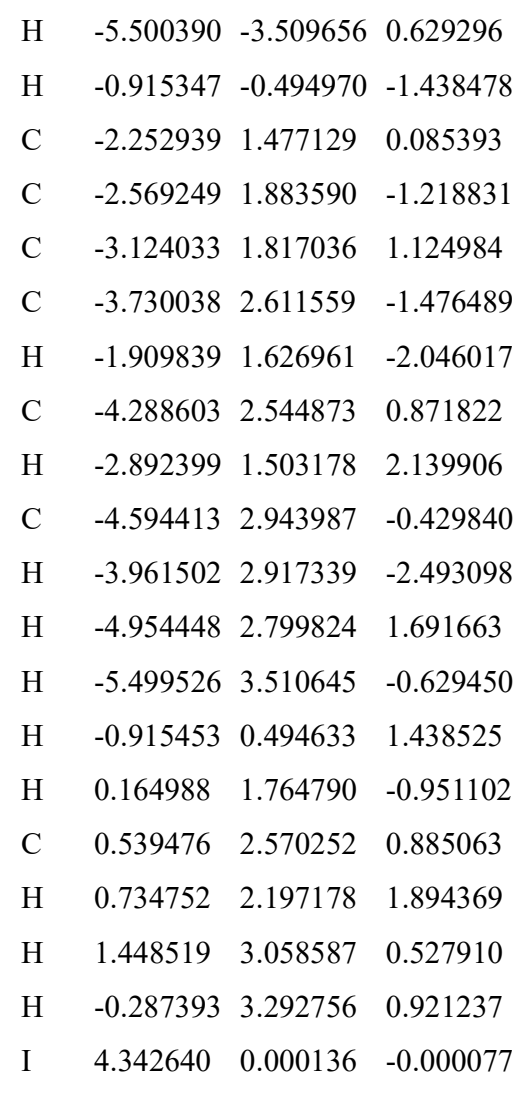

\section{INT1}

$\begin{array}{llll}\text { C } & -2.544796 & -0.721078 & -0.025947\end{array}$

$\begin{array}{llll}\text { C } & -3.807681 & -0.139153 & -0.198651\end{array}$

$\begin{array}{lllll}\text { C } & -2.313071 & -2.018744 & -0.448571\end{array}$

$\begin{array}{llll}\text { C } & -4.866943 & -0.833276 & -0.770903\end{array}$

$\begin{array}{lllll}\text { C } & -3.368014 & -2.738877 & -1.029363\end{array}$

H $\quad-1.325853 \quad-2.459730 \quad-0.346901$

C $\quad-4.625570-2.151350-1.183621$

H $\quad-5.841983 \quad-0.374095 \quad-0.900030$

$\mathrm{H} \quad-3.202623 \quad-3.757447 \quad-1.367692$

H $\quad-5.433392 \quad-2.719747 \quad-1.636352$

$\begin{array}{llll}\text { C } & -2.551316 & 1.528606 & 0.775702\end{array}$

$\begin{array}{lllll}\text { O } & -2.251078 & 2.614938 & 1.246064\end{array}$

$\begin{array}{llll}\mathrm{N} & -3.791009 & 1.182815 & 0.279670\end{array}$

$\begin{array}{llll}\text { C } & -4.912630 & 2.096455 & 0.228998\end{array}$

$\begin{array}{lllll}\mathrm{H} & -5.233148 & 2.254456 & -0.807404\end{array}$

$\begin{array}{lllll}\mathrm{H} & -5.757507 & 1.701885 & 0.805443\end{array}$

$\begin{array}{lllll}\mathrm{H} & -4.587278 & 3.044759 & 0.659691\end{array}$

$\begin{array}{lllll}\text { C } & -0.376427 & 0.598182 & -0.196917\end{array}$

$\begin{array}{lllll}\mathrm{O} & -0.320968 & 0.336195 & -1.387518\end{array}$

$\begin{array}{llll}\mathrm{N} & 0.681006 & 1.159555 & 0.482787\end{array}$

$\begin{array}{lllll}\text { C } & -1.638646 & 0.285757 & 0.630893\end{array}$

$\begin{array}{llll}\mathrm{H} & -1.350916 & -0.032845 & 1.639785\end{array}$ 


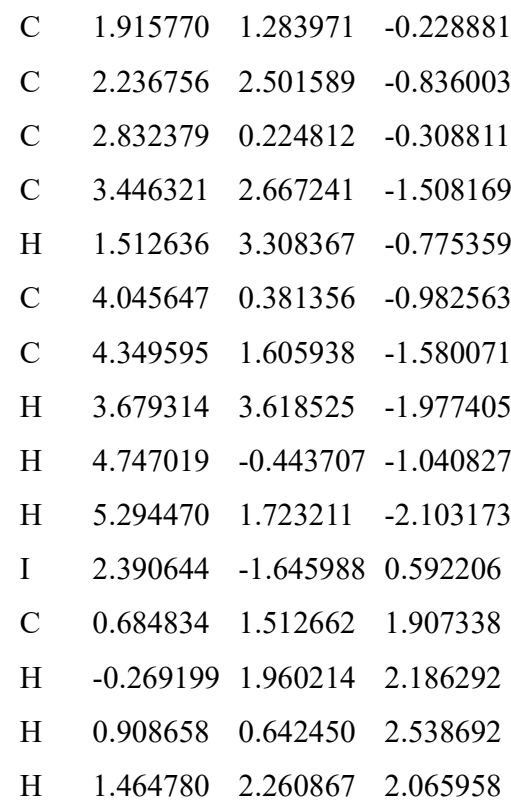

\section{INT2}

C $\quad-2.252358 \quad-0.332039 \quad 0.451925$

$\begin{array}{llll}\text { C } & -3.031854 & 0.560878 & -0.341690\end{array}$

$\begin{array}{lllll}\text { C } & -2.694255 & -1.651795 & 0.598287\end{array}$

$\begin{array}{llll}\text { C } & -4.203765 & 0.163673 & -0.976463\end{array}$

$\begin{array}{llll}\text { C } & -3.873534 & -2.058657 & -0.040927\end{array}$

H $\quad-2.120571 \quad-2.331623 \quad 1.219155$

C $\quad-4.623068-1.168334-0.820198$

$\mathrm{H} \quad-4.778988 \quad 0.866579 \quad-1.575849$

$\begin{array}{llll}\mathrm{H} & -4.215824 & -3.086014 & 0.076639\end{array}$

$\mathrm{H} \quad \begin{array}{llll}-5.538649 & -1.503382 & -1.304463\end{array}$

$\begin{array}{llll}\text { C } & -1.225019 & 1.760441 & 0.475733\end{array}$

$\begin{array}{llll}\text { O } & -0.568428 & 2.795035 & 0.694909\end{array}$

$\begin{array}{llll}\mathrm{N} & -2.410453 & 1.796627 & -0.325427\end{array}$

$\begin{array}{llll}\text { C } & -2.906810 & 2.999831 & -0.928156\end{array}$

$\mathrm{H} \quad-3.902886 \quad 3.268237 \quad-0.543213$

$\mathrm{H} \quad-2.980564 \quad 2.905598 \quad-2.022222$

H $\quad-2.196619 \quad 3.793073 \quad-0.678879$

$\begin{array}{llll}\text { C } & -0.101760 & -0.179734 & 1.783334\end{array}$

$\begin{array}{llll}\text { C } & 1.753831 & 0.944893 & 0.559740\end{array}$

$\begin{array}{llll}\text { C } & 2.774712 & 2.874359 & -0.534389\end{array}$

$\begin{array}{lllll}\text { O } & -0.299159 & -1.140991 & 2.542651\end{array}$

$\begin{array}{llll}\mathrm{N} & 1.200245 & 0.394447 & 1.747460\end{array}$

$\begin{array}{llll}\text { C } & 2.008125 & 0.199241 & -0.606285\end{array}$

$\begin{array}{llll}\text { C } & 3.002002 & 2.119321 & -1.686283\end{array}$

H $\quad 3.058567 \quad 3.923295 \quad-0.500736$

$\begin{array}{llll}\text { C } & 2.175459 & -0.115356 & 2.703259\end{array}$

$\begin{array}{llll}\text { C } & 2.613253 & 0.780416 & -1.725866\end{array}$

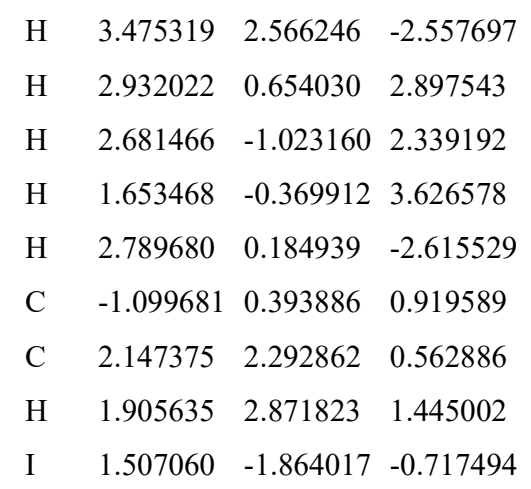

\section{INT3}

$\begin{array}{llll}\text { C } & -0.886651 & 2.439027 & 0.820235\end{array}$

$\begin{array}{llll}\text { C } & 0.146541 & 2.545082 & 1.789179\end{array}$

$\begin{array}{llll}\text { C } & -0.855221 & 3.277394 & -0.295855\end{array}$

C $\quad 1.218936 \quad 3.420046 \quad 1.635742$

$\begin{array}{llll}\text { C } & 0.216176 & 4.168758 & -0.455475\end{array}$

H $\quad-1.662061 \quad 3.231142 \quad-1.020393$

$\begin{array}{llll}\text { C } & 1.245014 & 4.235872 & 0.492401\end{array}$

$\begin{array}{llll}\text { H } & 2.002292 & 3.482999 & 2.386319\end{array}$

H $\quad 0.242671 \quad 4.822809 \quad-1.323050$

$\begin{array}{llll}\mathrm{H} & 2.065532 & 4.934798 & 0.353380\end{array}$

$\begin{array}{llll}\text { C } & -1.301740 & 0.905011 & 2.551548\end{array}$

$\begin{array}{llll}\text { O } & -1.772206 & 0.108728 & 3.369625\end{array}$

$\begin{array}{lllll}\mathrm{N} & -0.104938 & 1.632671 & 2.805076\end{array}$

C $\quad 0.606642 \quad 1.567006 \quad 4.058552$

$\mathrm{H} \quad 0.588798 \quad 2.535488 \quad 4.574716$

$\begin{array}{llll}\mathrm{H} & 1.655401 & 1.274424 & 3.909343\end{array}$

H $\quad 0.105099 \quad 0.819083 \quad 4.676530$

$\begin{array}{llll}\text { C } & -3.180267 & 1.282658 & 0.823618\end{array}$

$\begin{array}{lllll}\text { C } & -3.086769 & -1.187135 & 0.691441\end{array}$

$\begin{array}{lllll}\text { C } & -2.269153 & -3.305571 & 1.577828\end{array}$

$\begin{array}{llll}\text { O } & -3.817148 & 2.274477 & 0.481739\end{array}$

$\begin{array}{llll}\mathrm{N} & -3.801548 & 0.024389 & 0.893021\end{array}$

$\begin{array}{llll}\text { C } & -2.668132 & -1.605878 & -0.600036\end{array}$

$\begin{array}{llll}\text { C } & -1.819332 & -3.683512 & 0.309557\end{array}$

$\begin{array}{llll}\mathrm{H} & -2.128041 & -3.969890 & 2.425854\end{array}$

$\begin{array}{llll}\text { C } & -5.257309 & -0.053660 & 0.795675\end{array}$

$\begin{array}{llll}\text { C } & -2.023059 & -2.838577 & -0.783736\end{array}$

$\begin{array}{llll}\mathrm{H} & -1.335679 & -4.645182 & 0.159669\end{array}$

$\begin{array}{llll}\mathrm{H} & -5.633437 & -0.799735 & 1.503781\end{array}$

H $\quad-5.586558-0.324005 \quad-0.216203$

$\begin{array}{llll}\mathrm{H} & -5.662168 & 0.928857 & 1.038660\end{array}$

H $\quad-1.713231 \quad-3.142022-1.778214$

$\begin{array}{llll}\text { C } & -1.751395 & 1.320788 & 1.207346\end{array}$ 


\begin{tabular}{|c|c|c|c|}
\hline $\mathrm{C}$ & -2.888459 & -2.071712 & 1.766499 \\
\hline $\mathrm{H}$ & -3.191116 & -1.731857 & 2.747986 \\
\hline I & -3.193135 & -0.475057 & -2.327742 \\
\hline $\mathrm{Cu}$ & -0.695065 & -0.179201 & 0.177565 \\
\hline $\mathrm{N}$ & 0.785029 & 0.526406 & -1.250715 \\
\hline $\mathrm{C}$ & 2.012559 & -0.277671 & -1.069339 \\
\hline $\mathrm{C}$ & 2.134821 & -0.719237 & 0.416059 \\
\hline $\mathrm{N}$ & 0.904259 & -1.432956 & 0.837294 \\
\hline $\mathrm{H}$ & 0.824581 & -2.310154 & 0.321220 \\
\hline $\mathrm{C}$ & 0.884540 & -1.737354 & 2.282347 \\
\hline $\mathrm{H}$ & -0.073936 & -2.191689 & 2.529241 \\
\hline $\mathrm{H}$ & 1.710892 & -2.394283 & 2.584100 \\
\hline $\mathrm{H}$ & 0.952361 & -0.798063 & 2.830348 \\
\hline $\mathrm{C}$ & 3.410115 & -1.512538 & 0.649840 \\
\hline $\mathrm{C}$ & 3.567995 & -2.789458 & 0.094731 \\
\hline $\mathrm{C}$ & 4.454483 & -0.975208 & 1.408149 \\
\hline $\mathrm{C}$ & 4.744442 & -3.512118 & 0.291375 \\
\hline $\mathrm{H}$ & 2.769350 & -3.226203 & -0.502486 \\
\hline $\mathrm{C}$ & 5.635459 & -1.692898 & 1.605652 \\
\hline $\mathrm{H}$ & 4.345185 & 0.018555 & 1.835174 \\
\hline $\mathrm{C}$ & 5.783441 & -2.963822 & 1.047926 \\
\hline $\mathrm{H}$ & 4.851720 & -4.500803 & -0.146762 \\
\hline $\mathrm{H}$ & 6.438409 & -1.258976 & 2.195227 \\
\hline $\mathrm{H}$ & 6.701194 & -3.524732 & 1.201196 \\
\hline $\mathrm{H}$ & 2.164142 & 0.193186 & 1.024212 \\
\hline $\mathrm{C}$ & 3.274390 & 0.437408 & -1.528874 \\
\hline $\mathrm{C}$ & 3.594961 & 1.700149 & -1.009918 \\
\hline $\mathrm{C}$ & 4.138057 & -0.152686 & -2.456318 \\
\hline $\mathrm{C}$ & 4.758366 & 2.354730 & -1.413144 \\
\hline $\mathrm{H}$ & 2.936913 & 2.183975 & -0.291331 \\
\hline $\mathrm{C}$ & 5.305123 & 0.499667 & -2.859014 \\
\hline $\mathrm{H}$ & 3.901299 & -1.135179 & -2.857701 \\
\hline $\mathrm{C}$ & 5.618272 & 1.755962 & -2.337759 \\
\hline $\mathrm{H}$ & 4.991878 & 3.334405 & -1.004744 \\
\hline $\mathrm{H}$ & 5.966743 & 0.026903 & -3.579872 \\
\hline $\mathrm{H}$ & 6.524750 & 2.266752 & -2.650956 \\
\hline $\mathrm{H}$ & 1.877508 & -1.184840 & -1.673143 \\
\hline $\mathrm{H}$ & 0.928664 & 1.452008 & -0.841148 \\
\hline $\mathrm{C}$ & 0.424781 & 0.729445 & -2.663216 \\
\hline $\mathrm{H}$ & 0.125142 & -0.227834 & -3.099410 \\
\hline $\mathrm{H}$ & -0.432799 & 1.403403 & -2.701157 \\
\hline$H$ & 1.251026 & 1.152502 & -3.2511 \\
\hline
\end{tabular}

\section{INT4}

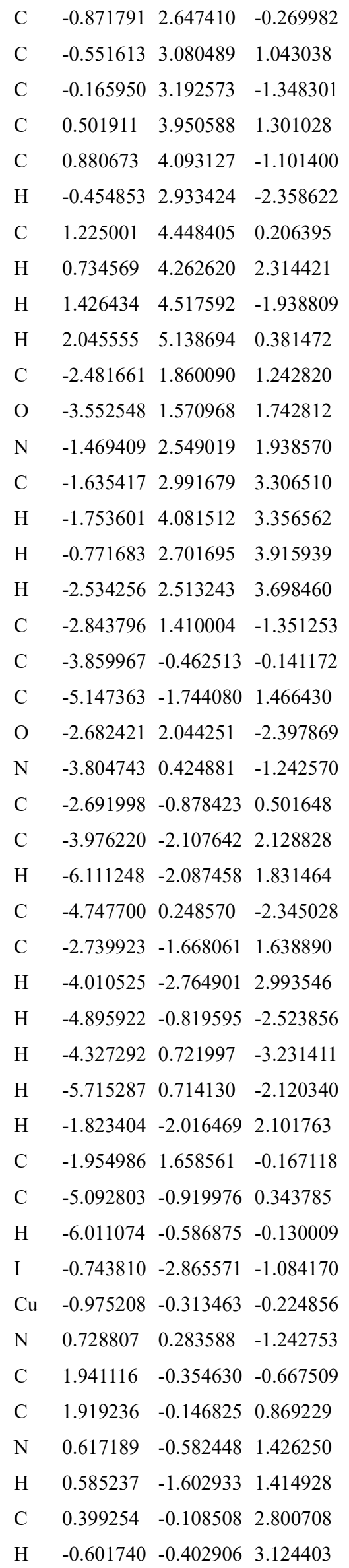

$\begin{array}{llll}\text { C } & -0.551613 & 3.080489 & 1.043038\end{array}$

C $\quad-0.165950 \quad 3.192573 \quad-1.348301$

$\begin{array}{llll}\text { C } & 0.501911 & 3.950588 & 1.301028\end{array}$

$\begin{array}{llll}\text { C } & 0.880673 & 4.093127 & -1.101400\end{array}$

$\mathrm{H} \quad-0.4548532 .933424 \quad-2.358622$

$\begin{array}{llll}\text { C } & 1.225001 & 4.448405 & 0.206395\end{array}$

$\mathrm{H} \quad 0.734569 \quad 4.262620 \quad 2.314421$

H $\quad 1.426434 \quad 4.517592 \quad-1.938809$

$\begin{array}{llll}\text { H } & 2.045555 & 5.138694 & 0.381472\end{array}$

C $\quad-2.481661 \quad 1.860090 \quad 1.242820$

C $\quad-1.635417 \quad 2.991679 \quad 3.306510$

$-1.753601 \quad 4.081512 \quad 3.356562$

$\begin{array}{llll}\text { H } & -2.534256 & 2.513243 & 3.698460\end{array}$

$\begin{array}{llll}\text { C } & -2.843796 & 1.410004 & -1.351253\end{array}$

$\begin{array}{llll}\text { C } & -3.859967 & -0.462513 & -0.141172\end{array}$

C $\quad-5.147363 \quad-1.744080 \quad 1.466430$

$\begin{array}{lllll}\text { O } & -2.682421 & 2.044251 & -2.397869\end{array}$

$\mathrm{N} \quad \begin{array}{llll}-3.804743 & 0.424881 & -1.242570\end{array}$

$\begin{array}{lll}-2.691998 & -0.878423 & 0.501648\end{array}$

$\begin{array}{llll}\text { C } & -3.976220 & -2.107642 & 2.128828\end{array}$

H $\quad-6.111248 \quad-2.087458 \quad 1.831464$

$\begin{array}{llll}\text { C } & -4.747700 & 0.248570 & -2.345028\end{array}$

$\begin{array}{llll}\text { C } & -2.739923 & -1.668061 & 1.638890\end{array}$

H $\quad-4.010525 \quad-2.7649012 .993546$

H $\quad-4.895922 \quad-0.819595 \quad-2.523856$

H $\quad-4.327292 \quad 0.721997 \quad-3.231411$

$\begin{array}{llll}\text { H } & -5.715287 & 0.714130 & -2.120340\end{array}$

H $\quad-1.823404 \quad-2.0164692 .101763$

$\begin{array}{llll}\text { C } & -1.954986 & 1.658561 & -0.167118\end{array}$

$\begin{array}{llll}\text { C } & -5.092803 & -0.919976 & 0.343785\end{array}$

H $\quad-6.011074 \quad-0.586875-0.130009$

$\mathrm{Cu} \quad-0.975208-0.313463-0.224856$

$\begin{array}{llll}\mathrm{N} & 0.728807 & 0.283588 & -1.242753\end{array}$

$\begin{array}{lll}1.941116 & -0.354630 & -0.667509\end{array}$

$\begin{array}{llll}\text { C } & 1.919236 & -0.146825 & 0.869229\end{array}$

$\begin{array}{llll}\mathrm{N} & 0.617189 & -0.582448 & 1.426250\end{array}$

$\begin{array}{llll}\text { C } & 0.399254 & -0.108508 & 2.800708\end{array}$

$\begin{array}{llll}\mathrm{H} & -0.601740 & -0.402906 & 3.124403\end{array}$ 


$$
\begin{array}{llll}
\mathrm{H} & 1.145796 & -0.494156 & 3.508920 \\
\mathrm{H} & 0.443374 & 0.982445 & 2.800394 \\
\mathrm{C} & 3.111060 & -0.821287 & 1.528499 \\
\mathrm{C} & 3.241947 & -2.217016 & 1.487129 \\
\mathrm{C} & 4.092771 & -0.068478 & 2.179212 \\
\mathrm{C} & 4.334138 & -2.843826 & 2.086069 \\
\mathrm{H} & 2.491623 & -2.819349 & 0.977667 \\
\mathrm{C} & 5.188597 & -0.693134 & 2.778587 \\
\mathrm{H} & 4.003934 & 1.014940 & 2.205735 \\
\mathrm{C} & 5.311256 & -2.082625 & 2.733796 \\
\mathrm{H} & 4.424368 & -3.925899 & 2.044426 \\
\mathrm{H} & 5.944825 & -0.094172 & 3.278852 \\
\mathrm{H} & 6.162930 & -2.571008 & 3.199345 \\
\mathrm{H} & 1.974595 & 0.933616 & 1.053660 \\
\mathrm{C} & 3.236202 & 0.166122 & -1.266708 \\
\mathrm{C} & 3.545239 & 1.533176 & -1.230190 \\
\mathrm{C} & 4.149944 & -0.718964 & -1.846868 \\
\mathrm{C} & 4.744638 & 2.002548 & -1.765142 \\
\mathrm{H} & 2.848370 & 2.241174 & -0.788602 \\
\mathrm{C} & 5.353971 & -0.252384 & -2.377992 \\
\mathrm{H} & 3.918981 & -1.780744 & -1.875050 \\
\mathrm{C} & 5.654098 & 1.110234 & -2.339060 \\
\mathrm{H} & 4.969339 & 3.065478 & -1.732961 \\
\mathrm{H} & 6.054734 & -0.953306 & -2.823305 \\
\mathrm{H} & 6.589354 & 1.475721 & -2.754304 \\
\mathrm{H} & 1.847981 & -1.422763 & -0.878216 \\
\mathrm{H} & 0.777195 & 1.283198 & -1.038560 \\
\mathrm{H} & 0.602682 & 0.151248 & -2.712804 \\
\mathrm{H} & -0.339742 & 0.616310 & -3.013456 \\
\mathrm{H} & 0.632451 & -3.231715
\end{array}
$$

$2 a$

$$
\begin{array}{llll}
\mathrm{C} & -1.195805 & -0.526128 & -0.489166 \\
\mathrm{C} & -2.337131 & -0.060808 & 0.180505 \\
\mathrm{C} & -1.298796 & -1.540811 & -1.425401 \\
\mathrm{C} & -3.598087 & -0.588412 & -0.072954 \\
\mathrm{C} & -2.563015 & -2.086804 & -1.695650 \\
\mathrm{H} & -0.413319 & -1.906945 & -1.937678 \\
\mathrm{C} & -3.693232 & -1.611073 & -1.027623 \\
\mathrm{H} & -4.477962 & -0.224103 & 0.447763 \\
\mathrm{H} & -2.661611 & -2.881986 & -2.428498 \\
\mathrm{H} & -4.667204 & -2.040188 & -1.246219 \\
\mathrm{C} & -0.618973 & 1.178656 & 1.088352 \\
\mathrm{O} & -0.025169 & 1.971420 & 1.790808 \\
\mathrm{~N} & -1.981189 & 0.939747 & 1.095307 \\
\mathrm{C} & -2.906225 & 1.645570 & 1.956332 \\
\mathrm{H} & -3.649787 & 2.186063 & 1.359363 \\
\mathrm{H} & -3.424658 & 0.946695 & 2.623569 \\
\mathrm{H} & -2.327496 & 2.355652 & 2.549259 \\
\mathrm{C} & 0.000001 & 0.251264 & 0.000007 \\
\mathrm{C} & 1.195816 & -0.526106 & 0.489189 \\
\mathrm{C} & 0.618960 & 1.178650 & -1.088351 \\
\mathrm{C} & 2.337132 & -0.060799 & -0.180509 \\
\mathrm{C} & 1.298822 & -1.540764 & 1.425450 \\
\mathrm{O} & 0.025141 & 1.971377 & -1.790835 \\
\mathrm{~N} & 1.981174 & 0.939727 & -1.095337 \\
\mathrm{C} & 3.598094 & -0.588390 & 0.072950 \\
\mathrm{C} & 2.563048 & -2.086744 & 1.695699 \\
\mathrm{H} & 0.413353 & -1.906889 & 1.937747 \\
\mathrm{H} & 3.906196 & 1.645531 & -1.956393 \\
\mathrm{H} & -676969 & -1.611025 & 1.027646 \\
\mathrm{H} & -0.224089 & -0.447784 \\
\mathrm{H} & -2.881906 & 2.428566 \\
\mathrm{H} & 2.186134 & -1.359451
\end{array}
$$




\section{References}

1. H. Wu, Y.-P. He, L. Xu, D.-Y. Zhang, L.-Z. Gong, Angew. Chem. Int. Ed. 2014, 53, 3466-3469.

2. J. Wang, Y. Yuan, R. Xiong, D. Zhang-Negrerie, Y. Du, K. Zhao, Org. Lett. 2012, 14, $2210-$ 2213.

3. Gaussian 16, Revision A.03, Frisch, M. J.; Trucks, G. W.; Schlegel, H. B.; Scuseria, G. E.; Robb, M. A.; Cheeseman, J. R.; Scalmani, G.; Barone, V.; Petersson, G. A.; Nakatsuji, H.; Li, X.; Caricato, M.; Marenich, A. V.; Bloino, J.; Janesko, B. G.; Gomperts, R.; Mennucci, B.; Hratchian, H. P.; Ortiz, J. V.; Izmaylov, A. F.; Sonnenberg, J. L.; Williams-Young, D.; Ding, F.; Lipparini, F.; Egidi, F.; Goings, J.; Peng, B.; Petrone, A.; Henderson, T.; Ranasinghe, D.; Zakrzewski, V. G.; Gao, J.; Rega, N.; Zheng, G.; Liang, W.; Hada, M.; Ehara, M.; Toyota, K.; Fukuda, R.; Hasegawa, J.; Ishida, M.; Nakajima, T.; Honda, Y.; Kitao, O.; Nakai, H.; Vreven, T.; Throssell, K.; Montgomery, J. A., Jr.; Peralta, J. E.; Ogliaro, F.; Bearpark, M. J.; Heyd, J. J.; Brothers, E. N.; Kudin, K. N.; Staroverov, V. N.; Keith, T. A.; Kobayashi, R.; Normand, J.; Raghavachari, K.; Rendell, A. P.; Burant, J. C.; Iyengar, S. S.; Tomasi, J.; Cossi, M.; Millam, J. M.; Klene, M.; Adamo, C.; Cammi, R.; Ochterski, J. W.; Martin, R. L.; Morokuma, K.; Farkas, O.; Foresman, J. B.; Fox, D. J. Gaussian, Inc., Wallingford CT, 2016.

4. A. D. Becke, J. Chem. Phys. 1993, 98, 5648-5652.

5. P. C. Hariharan, J. A. Pople, Theor. Chim. Acta 1973, 28, 213-222.

6. M. M. Francl, W. J. Pietro, W. J. Hehre, J. S. Binkley, M. S. Gordon, D. J. DeFrees, J. A. Pople, J. Chem. Phys. 1982, 77, 3654-3665.

7. P. J. Hay, W. R. Wadt, J. Chem. Phys. 1985, 82, 299.

8. L. E. Roy, P. J. Hay, R. L. Martin, J. Chem. Theory Comput. 2008, 4, 1029.

9. A. W. Ehlers, M. Böhme, S. Dapprich, A. Gobbi, A. Höllwarth, V. Jonas, K. F. Köhler, R. Stegmann, A. Veldkamp, G. Frenking, Chem. Phys. Lett. 1993, 208, 111.

10. F. Weigend, R. Ahlrichs, Phys. Chem. Chem. Phys. 2005, 7,3297-3305.

11. A. D. McLean, G. S. Chandler, J. Chem. Phys. 1980, 72, 5639-5648.

12. R. Krishnan, J. S. Binkley, R. Seeger, J. A. Pople, J. Chem. Phys. 1980, 72, 650-654.

13. R. C. Binning, L. A. Curtiss, J. Comput. Chem. 1990, 11, 1206-1216.

14. A. V. Marenich, C. J. Cramer, D. G. Truhlar, J. Phys. Chem. B. 2009, 113, 6378-6396.

15. C. Y. Legault, CYLview, 1.0b, Universite de Sherbrooke, 2009, http://www.cylview.org.

\section{NMR and HPLC Spectra}



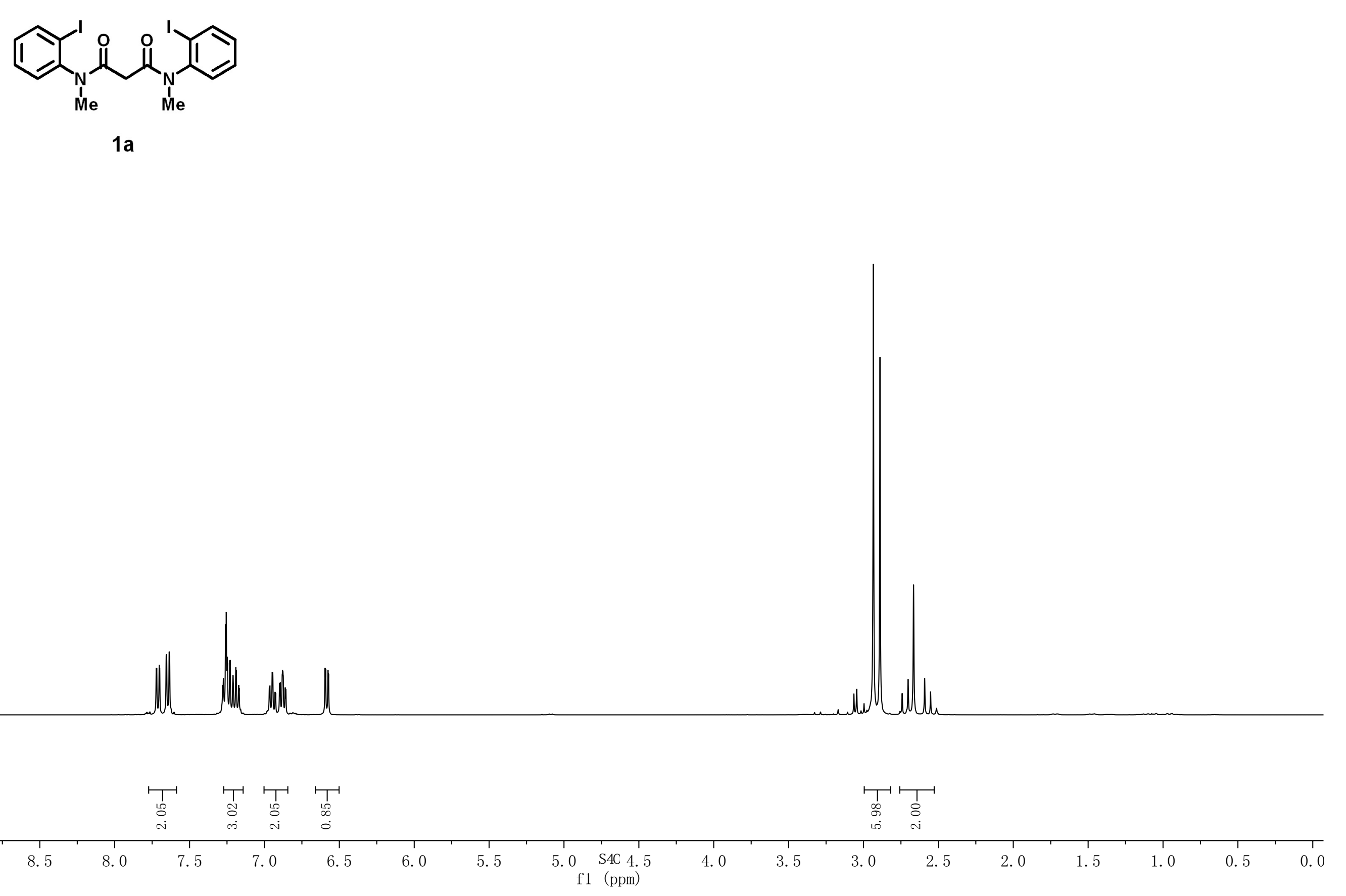


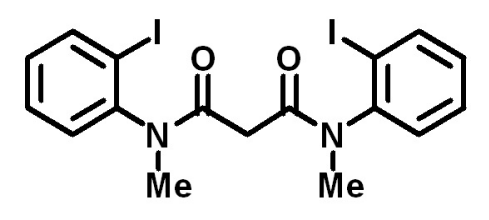

$1 a$
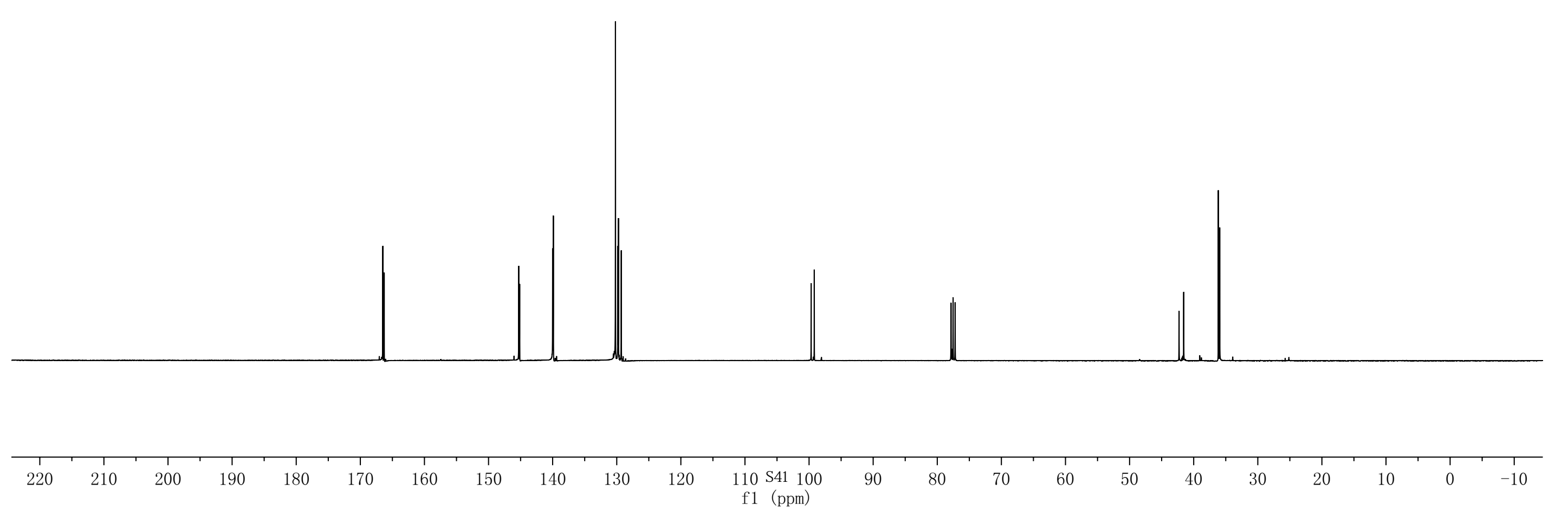


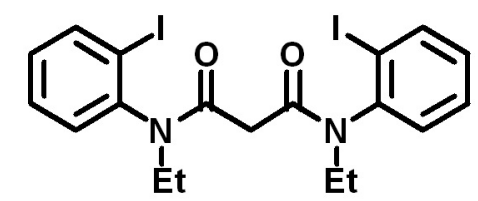

$1 \mathrm{~b}$

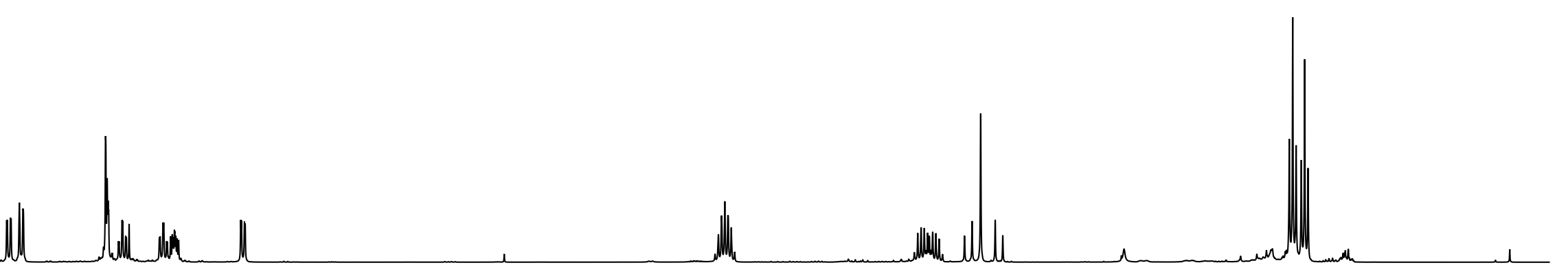

\begin{tabular}{|c|c|c|c|c|c|c|c|c|c|c|c|c|c|c|c|c|c|c|c|c|}
\hline & & & & 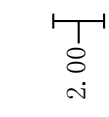 & 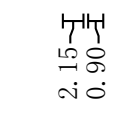 & $\begin{array}{l}1 \\
1 \\
8 \\
i \\
i\end{array}$ & $\begin{array}{c}\urcorner_{\infty}^{\top} \\
\infty \\
0 \\
0\end{array}$ & & & & & 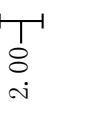 & & 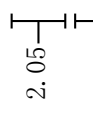 & & & & 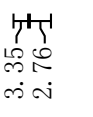 & & \\
\hline 1.0 & 9.5 & 9.0 & 8.5 & 8.0 & 7.5 & 7. 0 & 6.5 & 6.0 & 5.5 & $\begin{array}{l}\text { 5. } \$ 42 \\
\text { f1 (ppm) }\end{array}$ & 4.5 & 4.0 & 3.5 & 3.0 & 2.5 & 2.0 & 1.5 & 1.0 & 0.5 & 0.0 \\
\hline
\end{tabular}




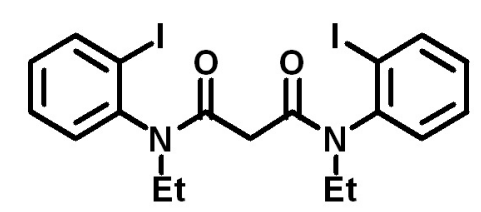

$1 \mathrm{~b}$

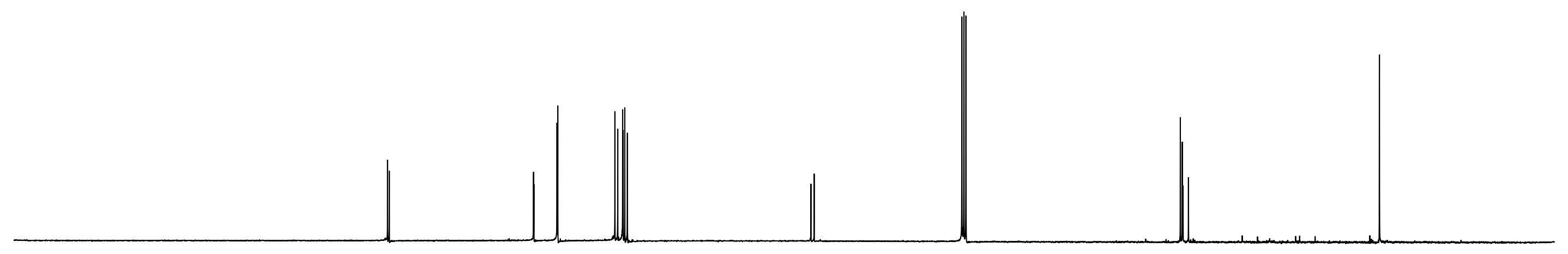


oreso

iber bis in 

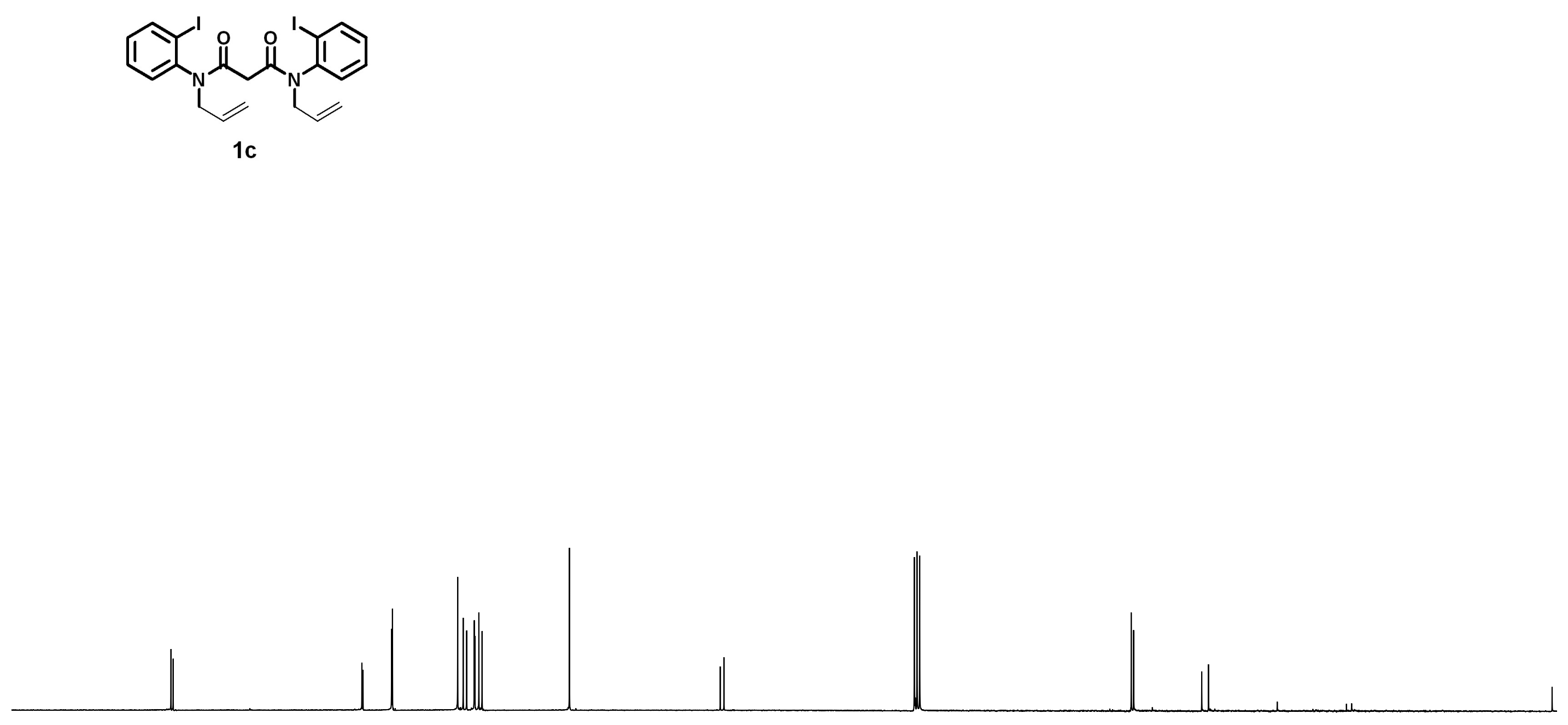

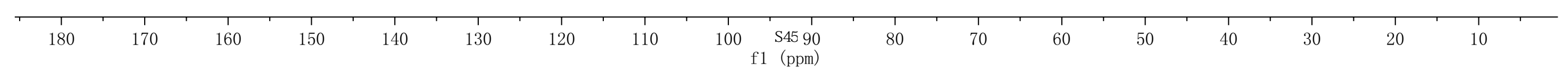



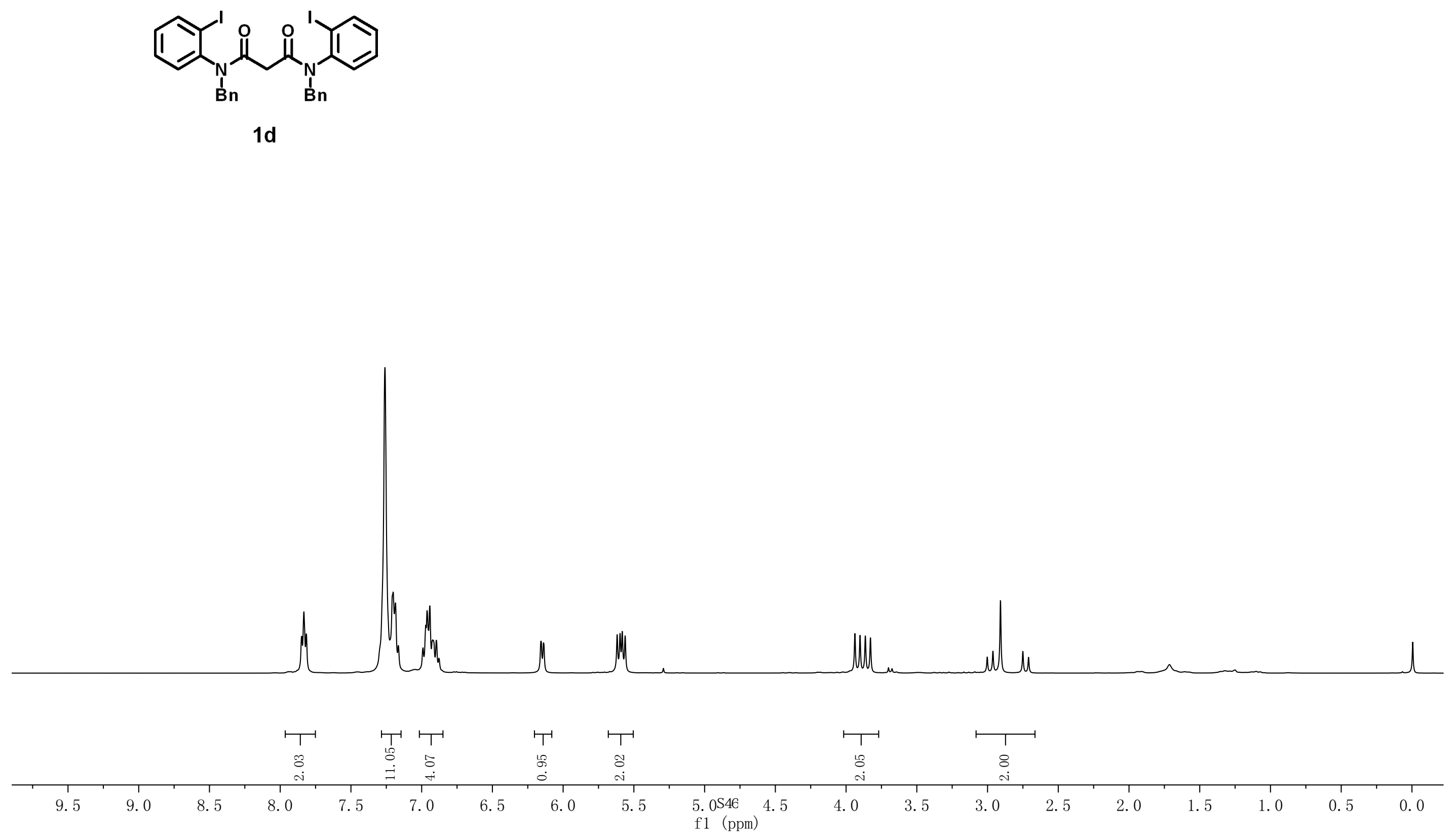


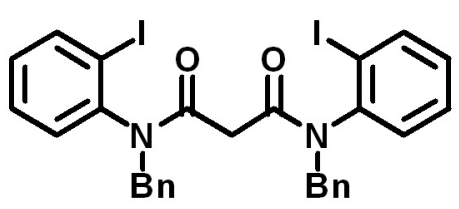

1d

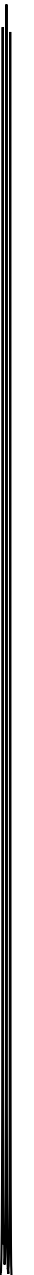



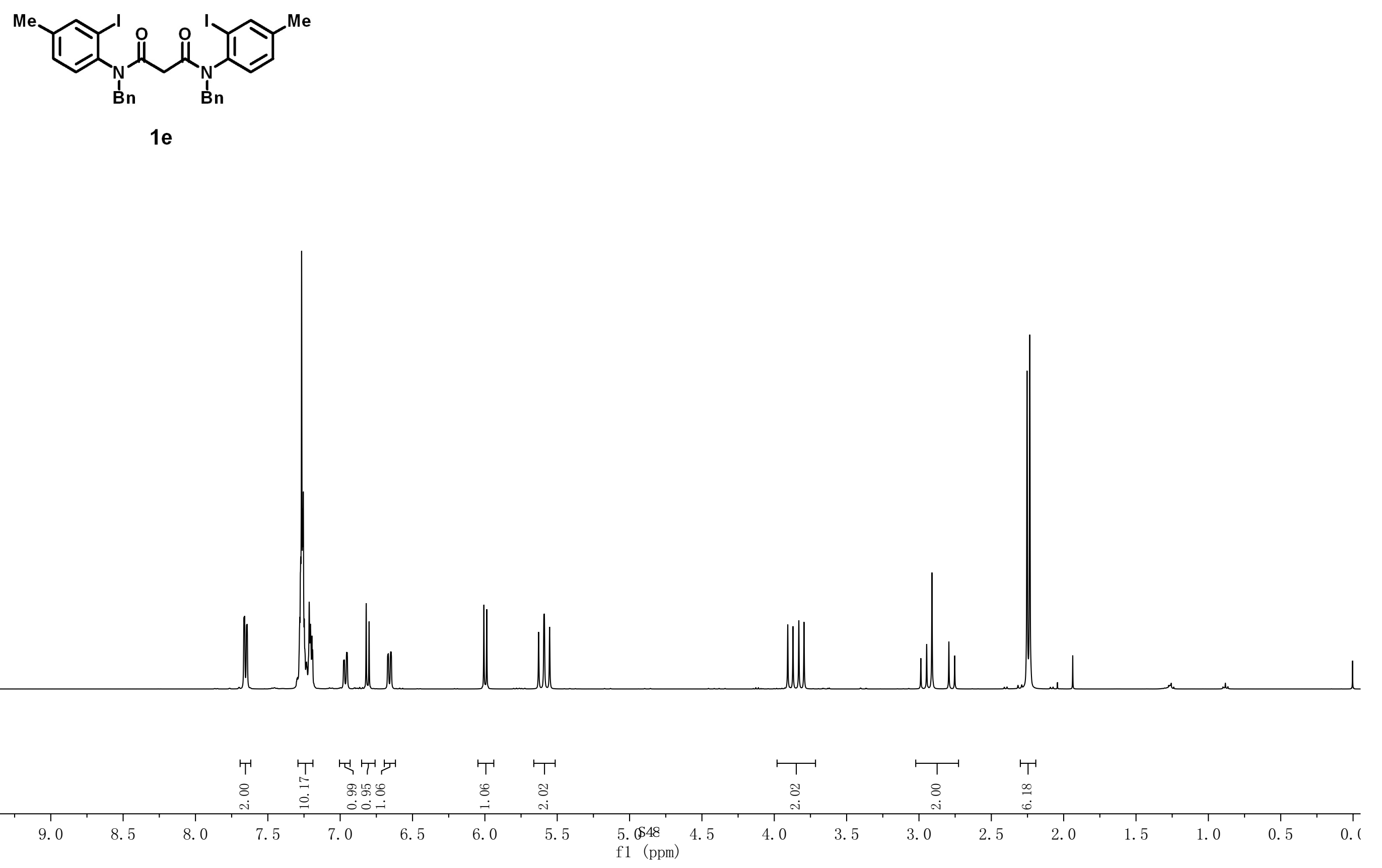

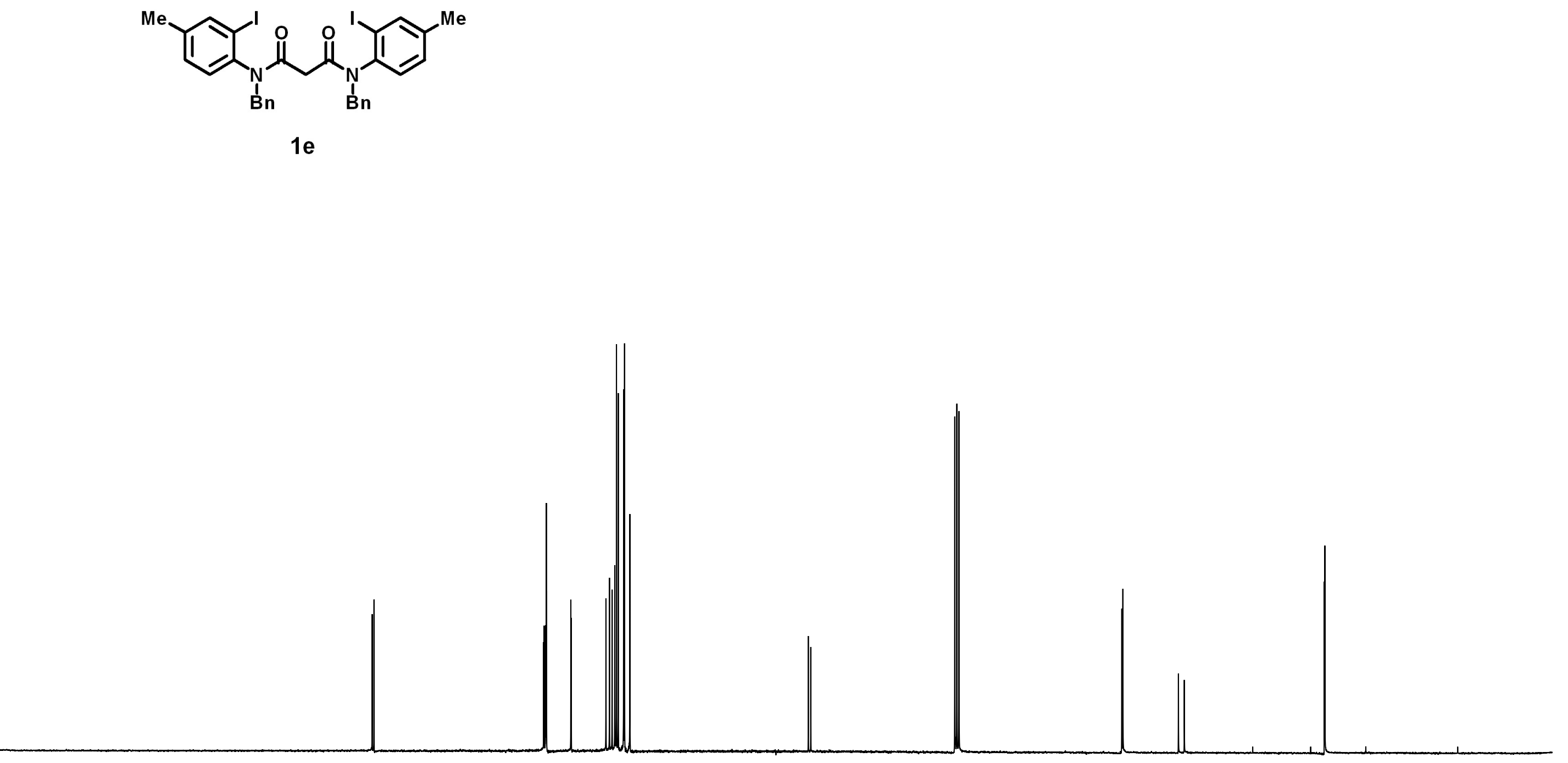

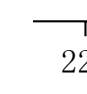



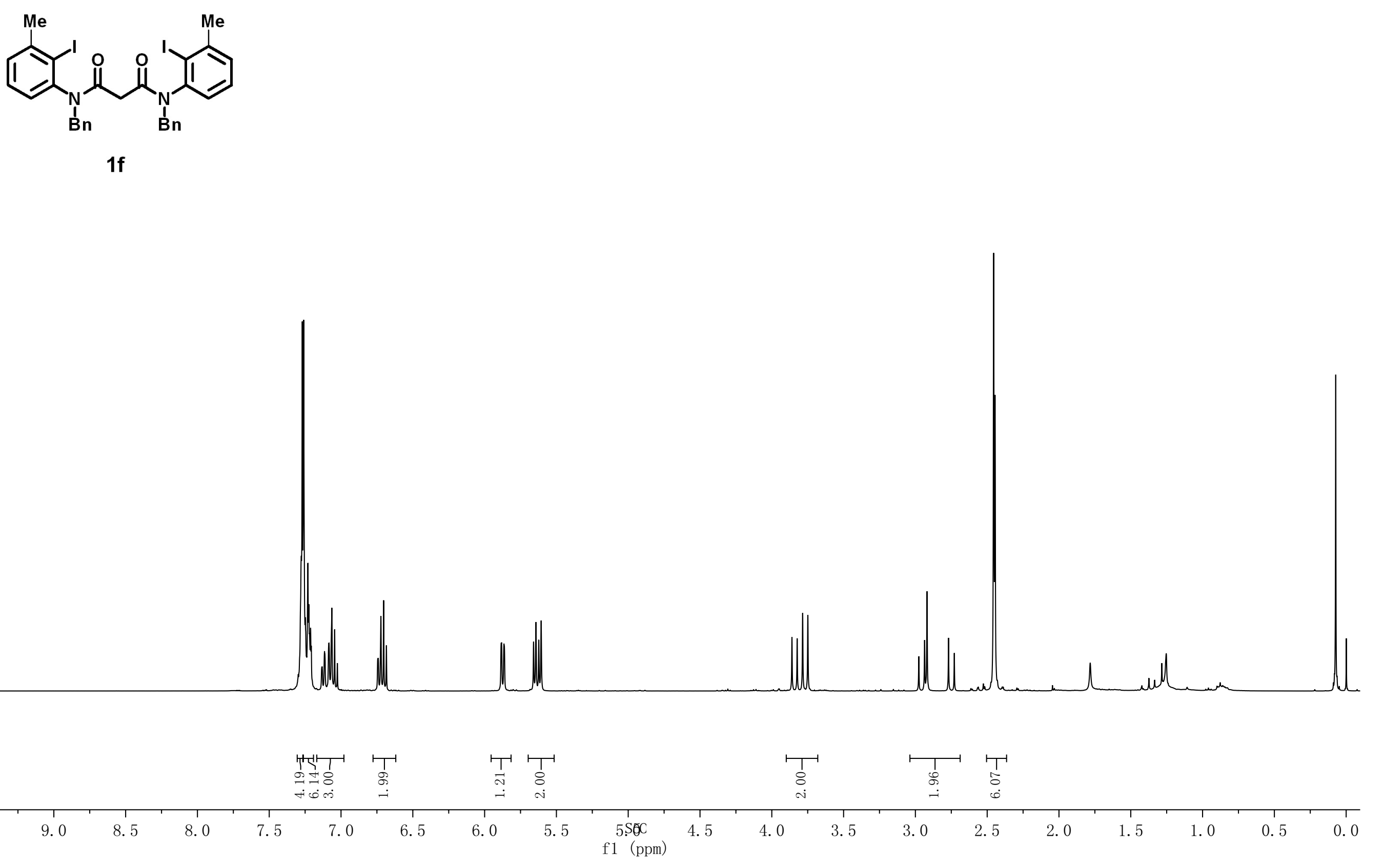

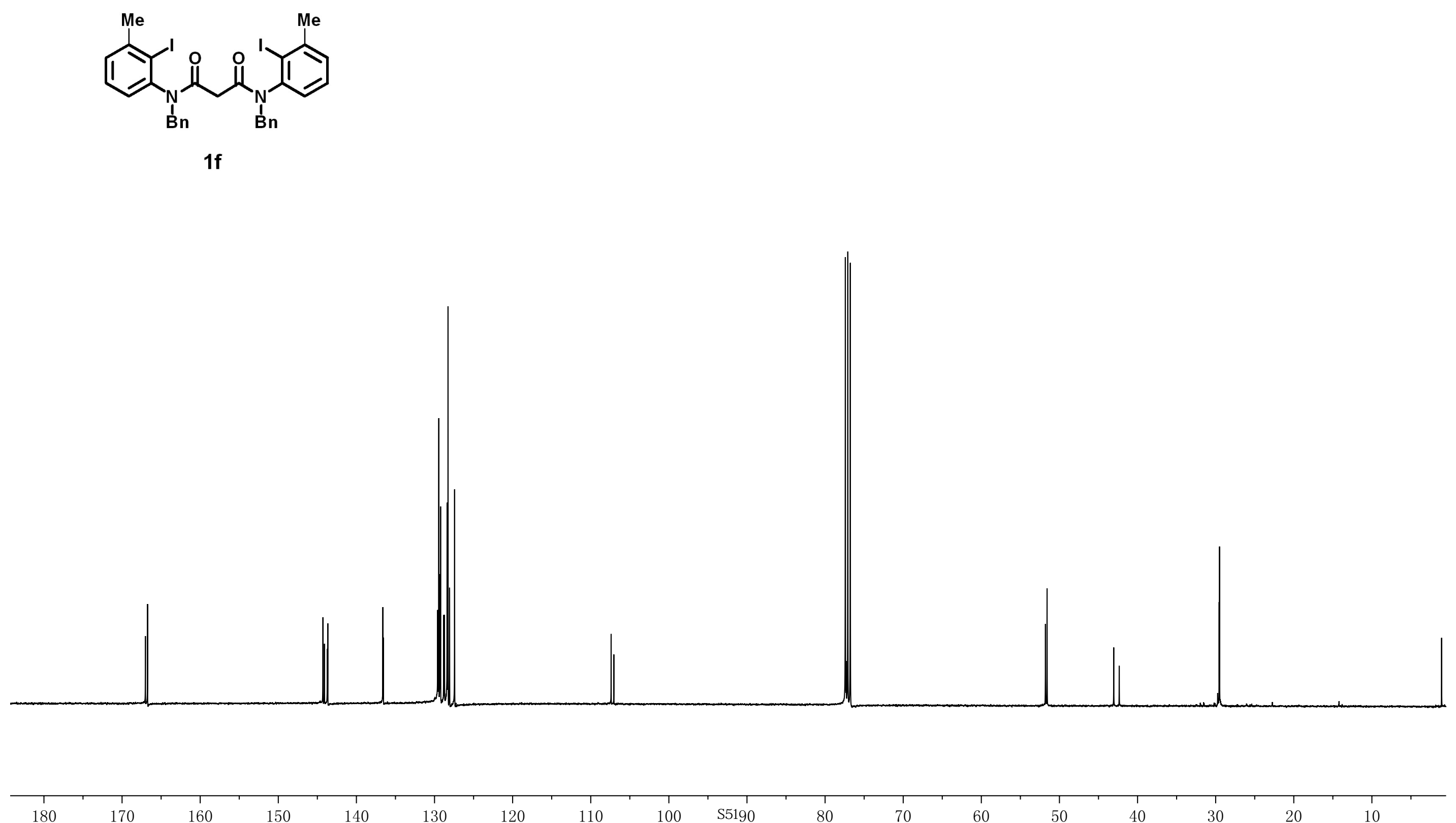

130

120 110 


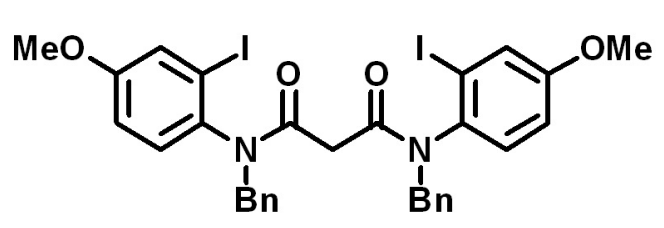

$1 \mathrm{~g}$
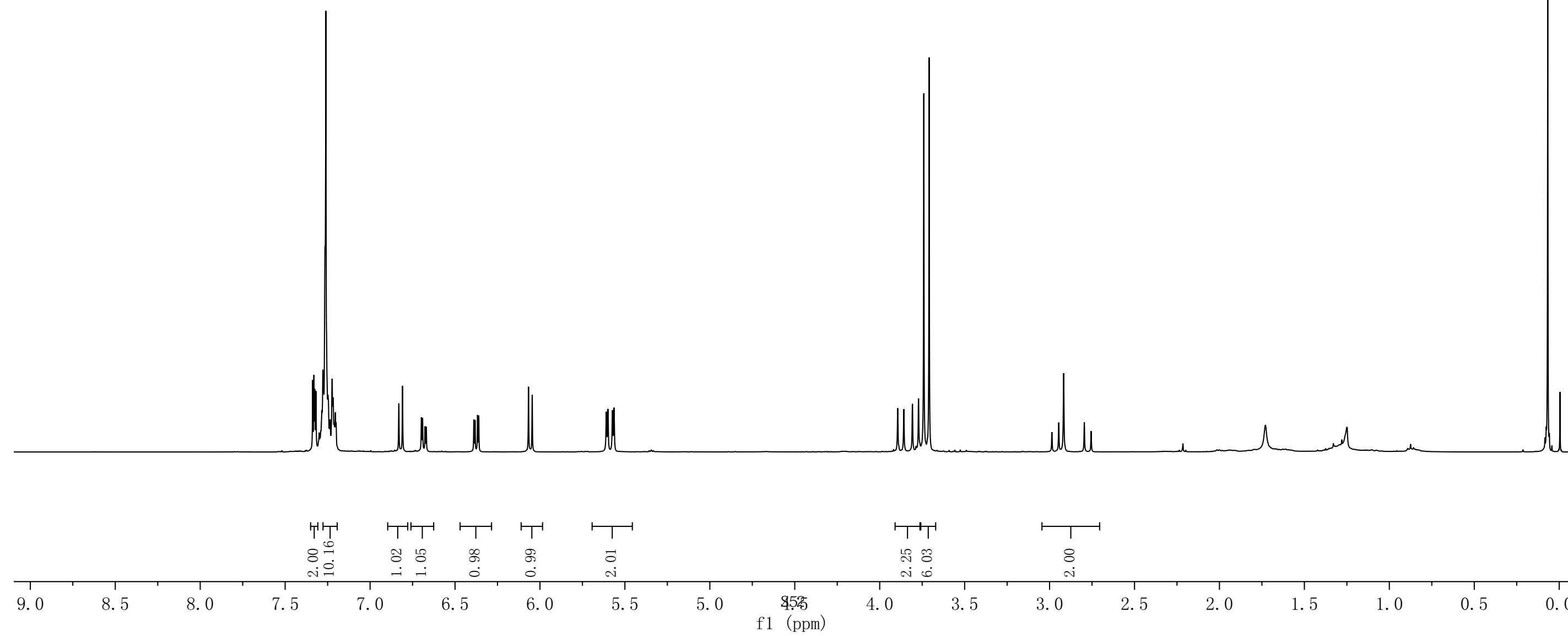
<smiles>COc1ccc(N(Cc2ccccc2)C(=O)CC(=O)N(Cc2ccccc2)c2ccc(I)cc2I)c(I)c1</smiles>

$1 \mathrm{~g}$
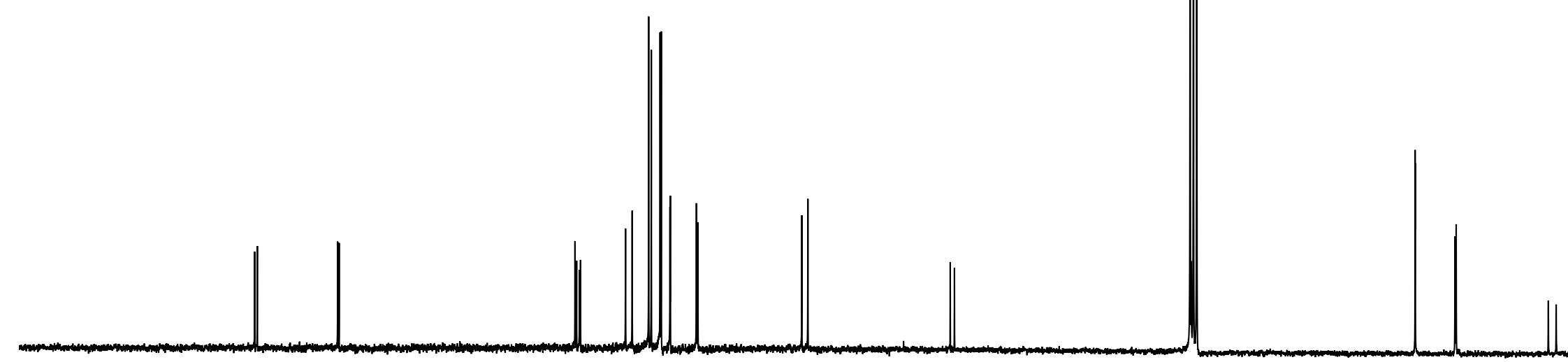

$170 \quad 160$

150

130

120 110

$100 \quad$ S53 90

80

70

60

50

10 

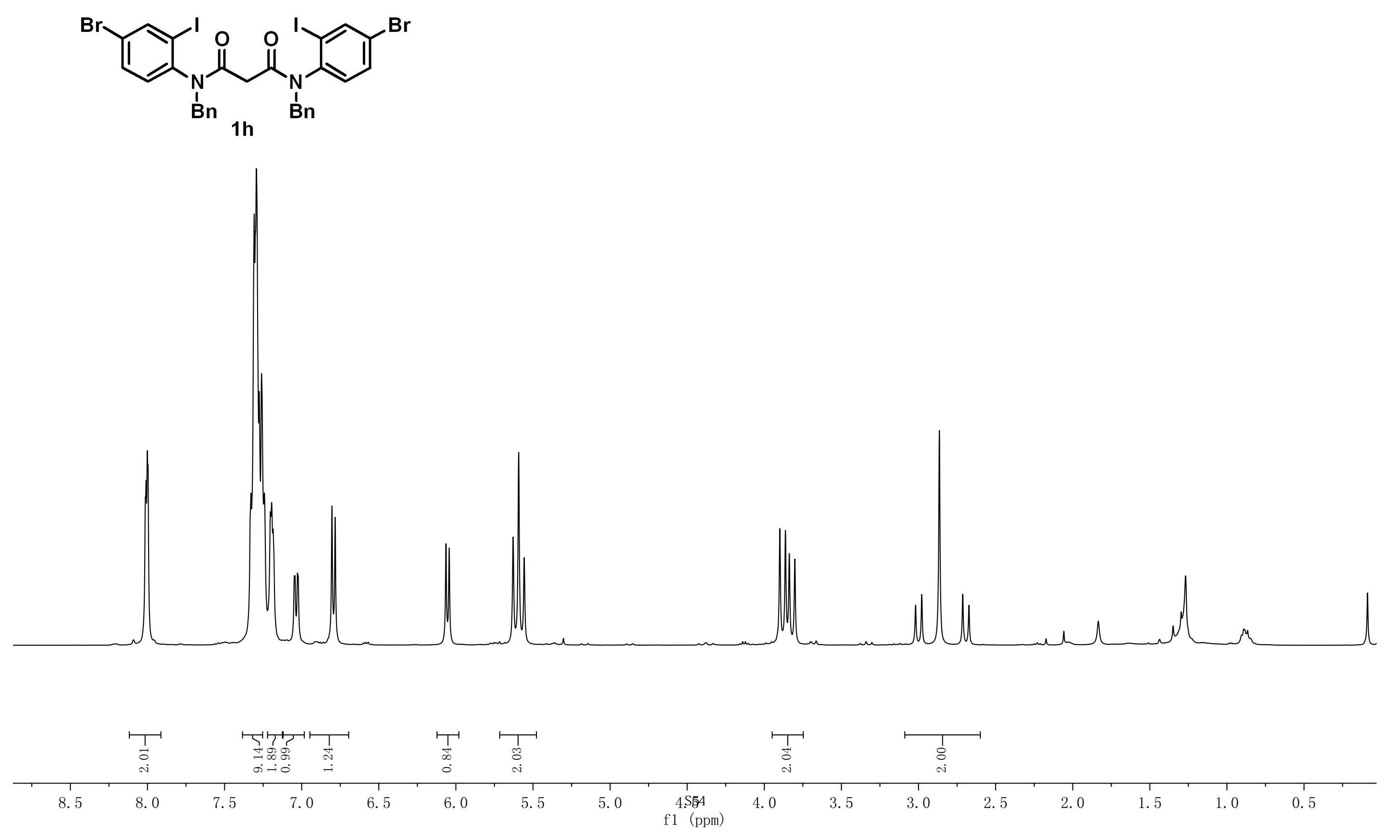


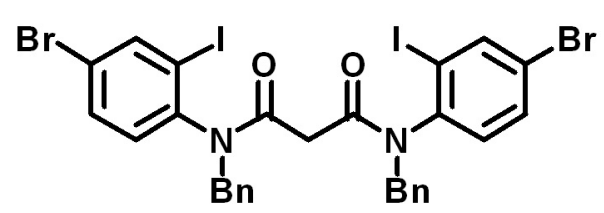

1h

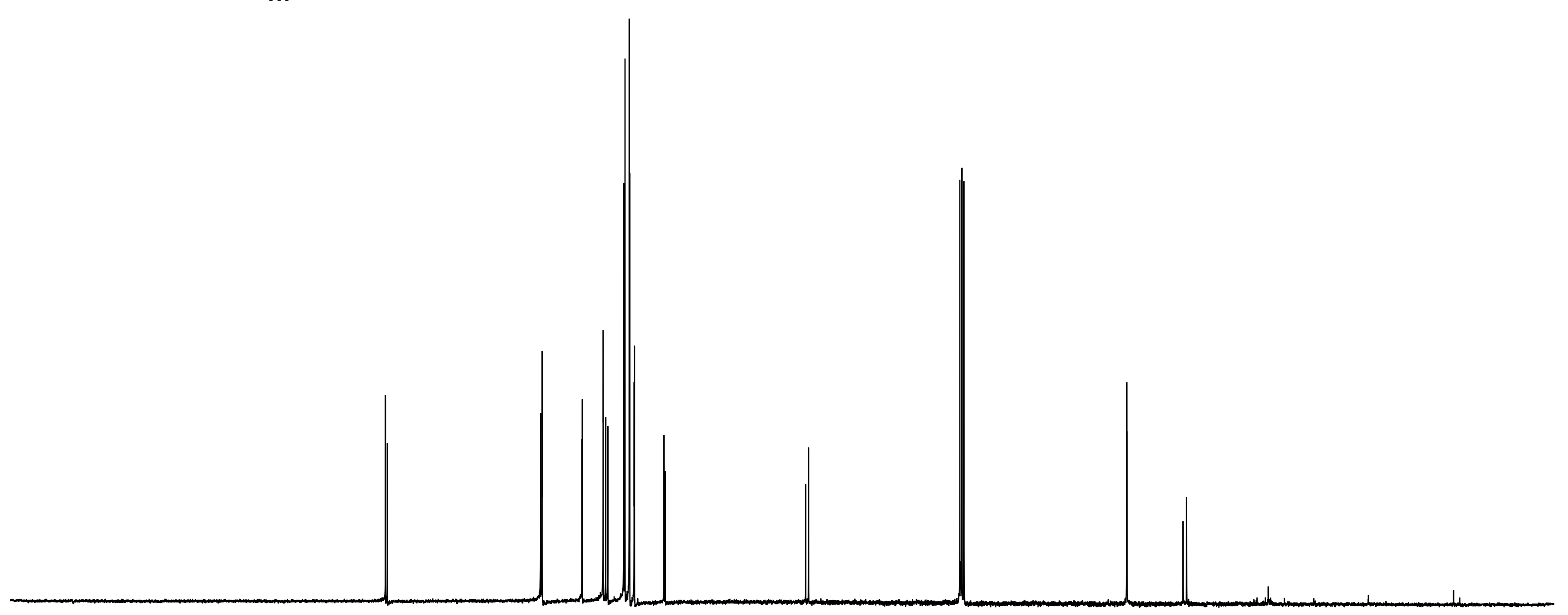



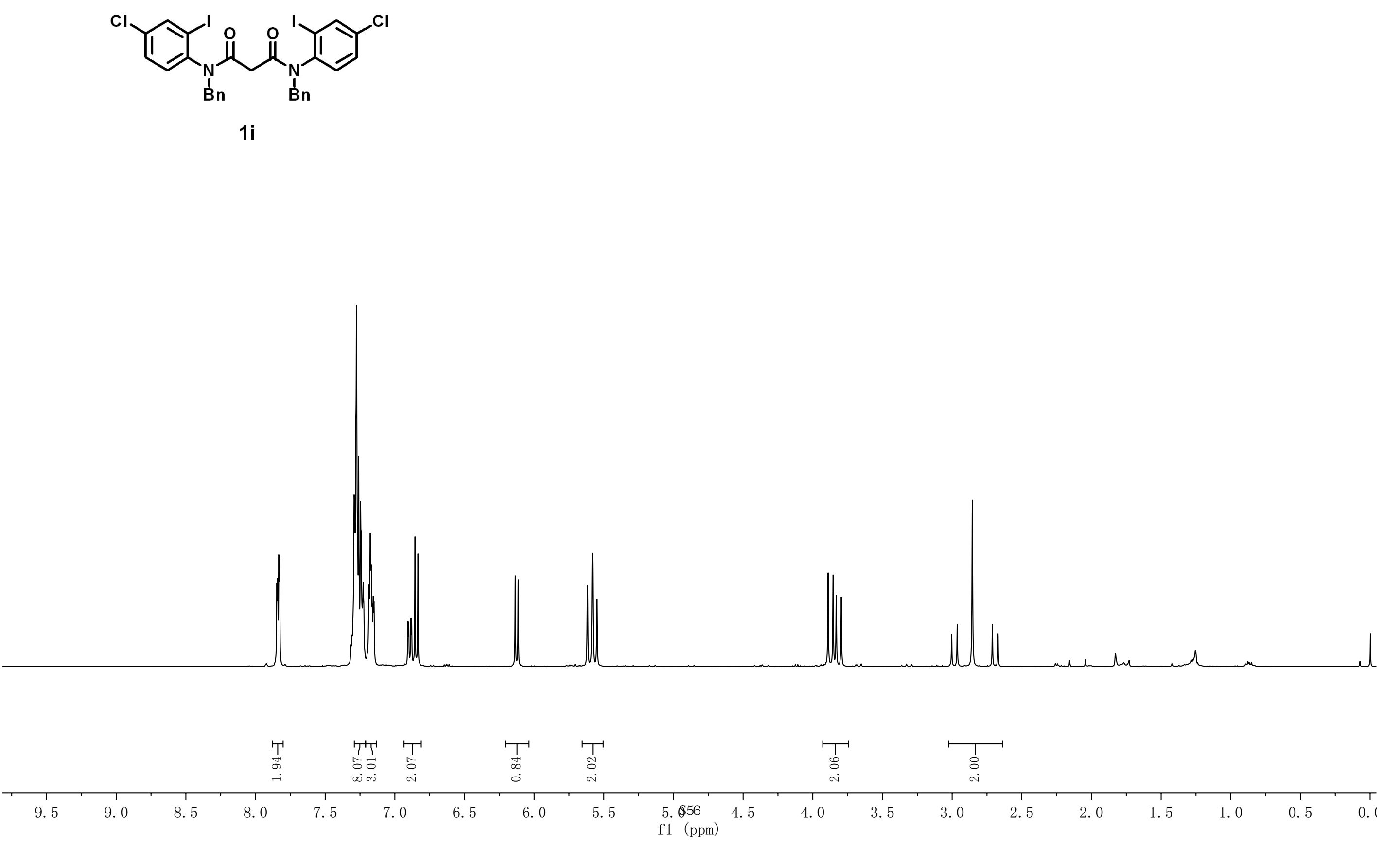

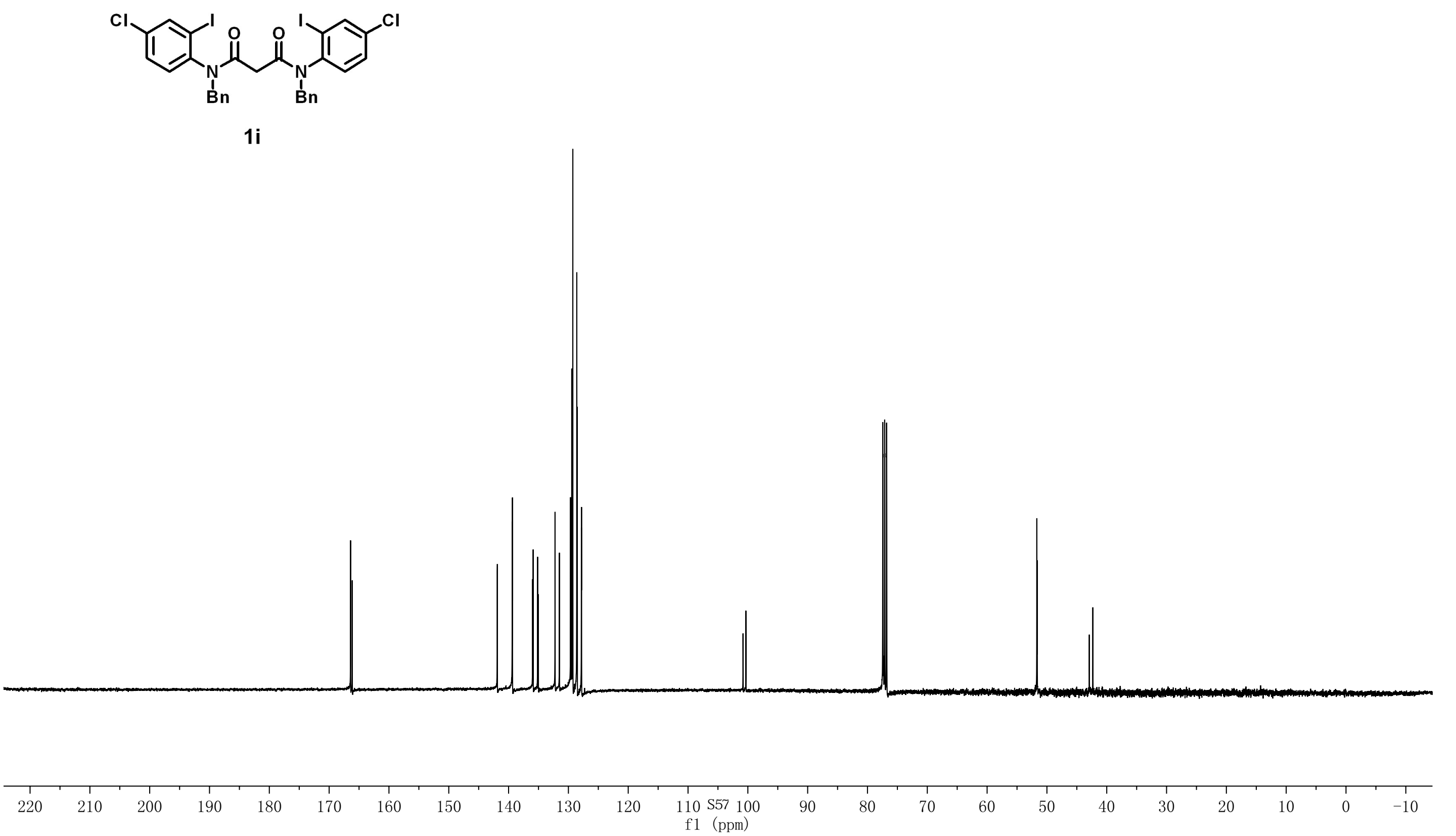

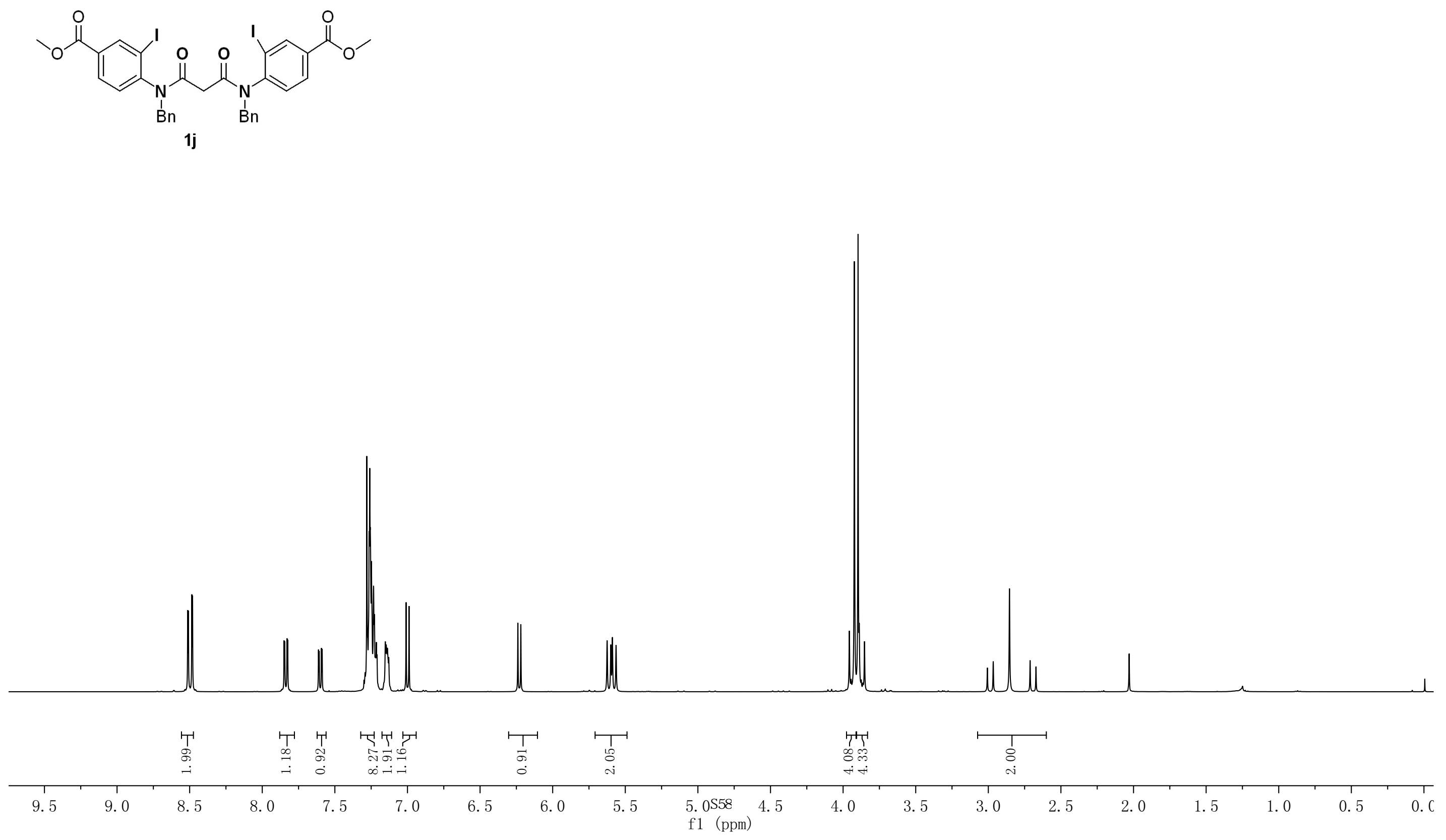

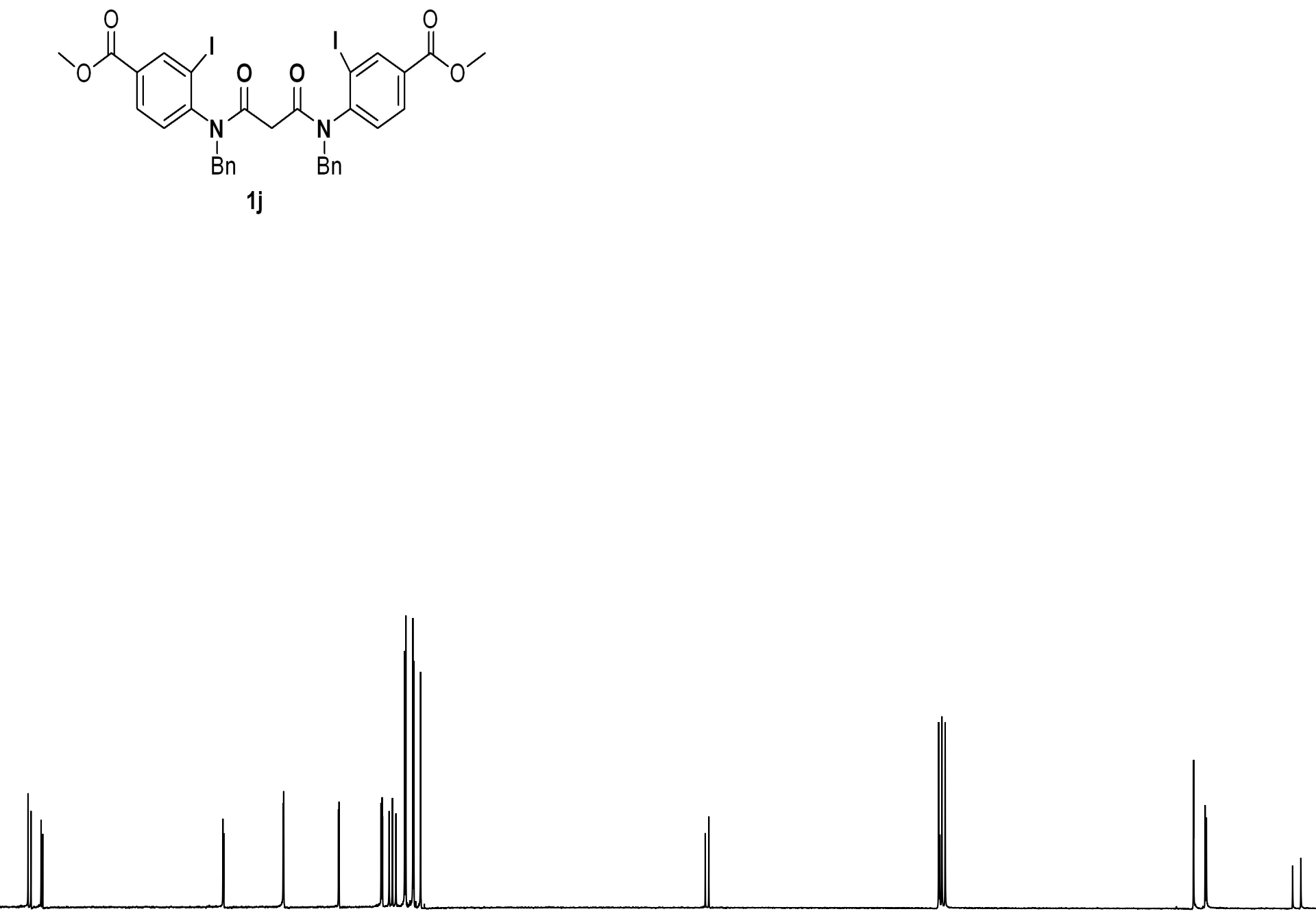

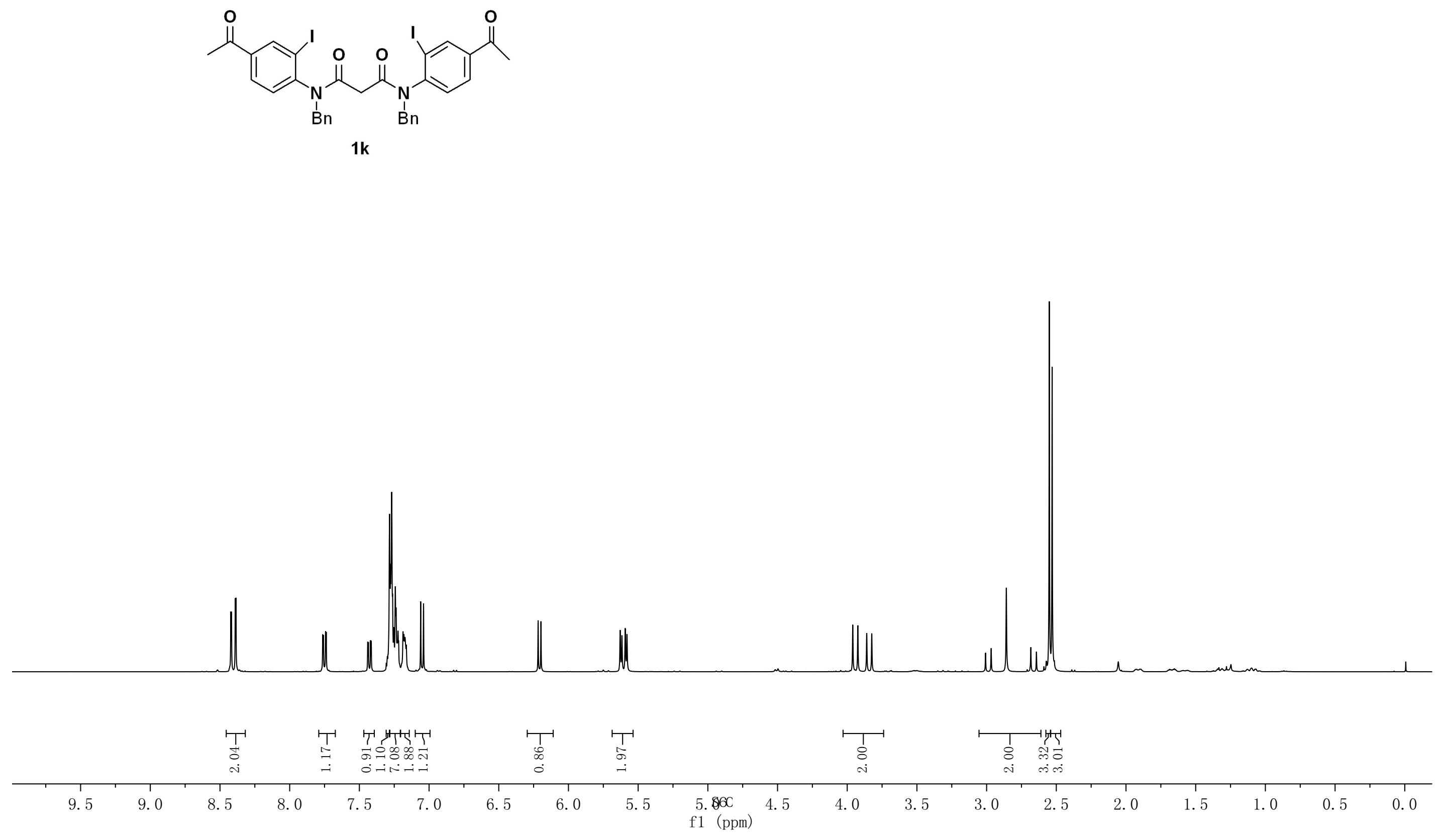

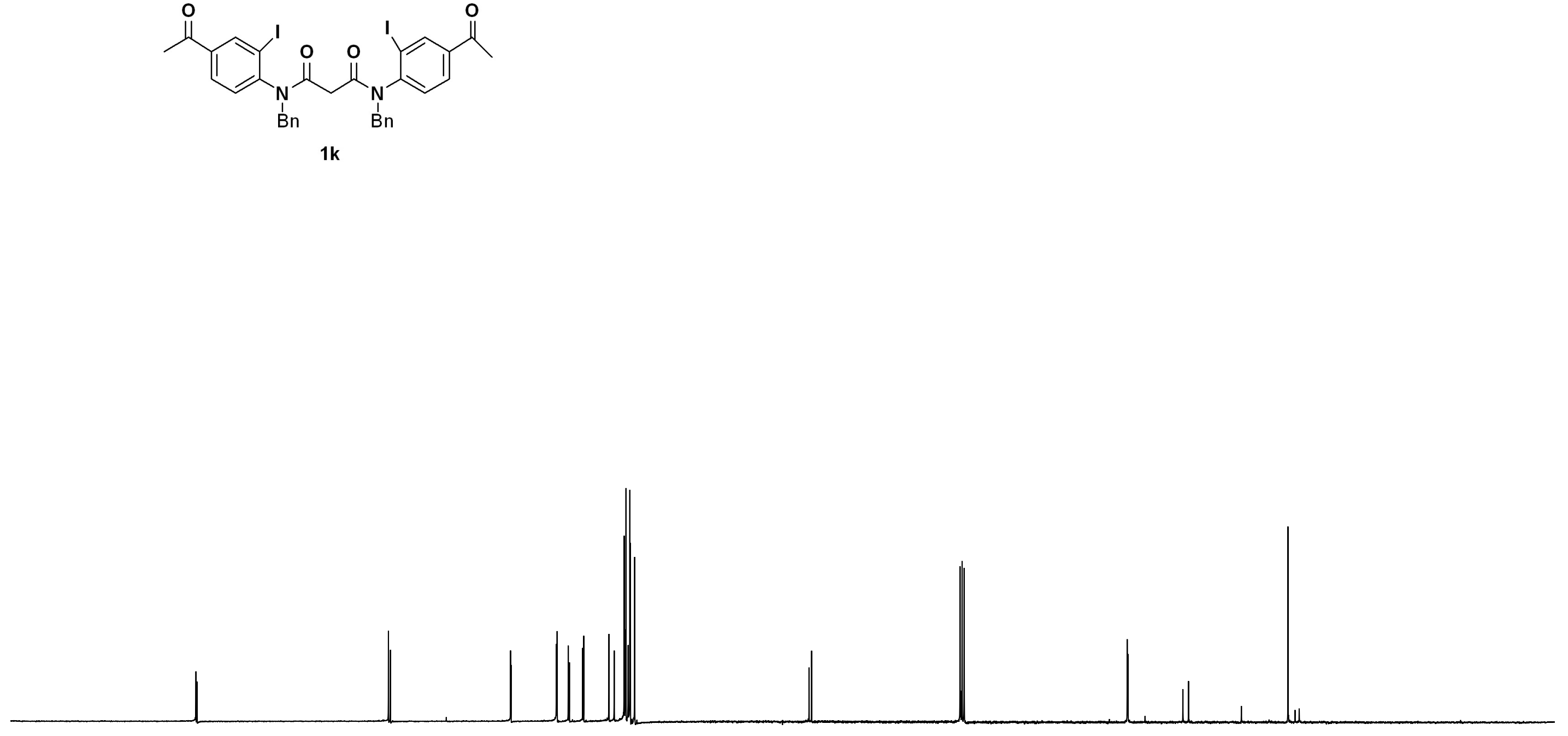

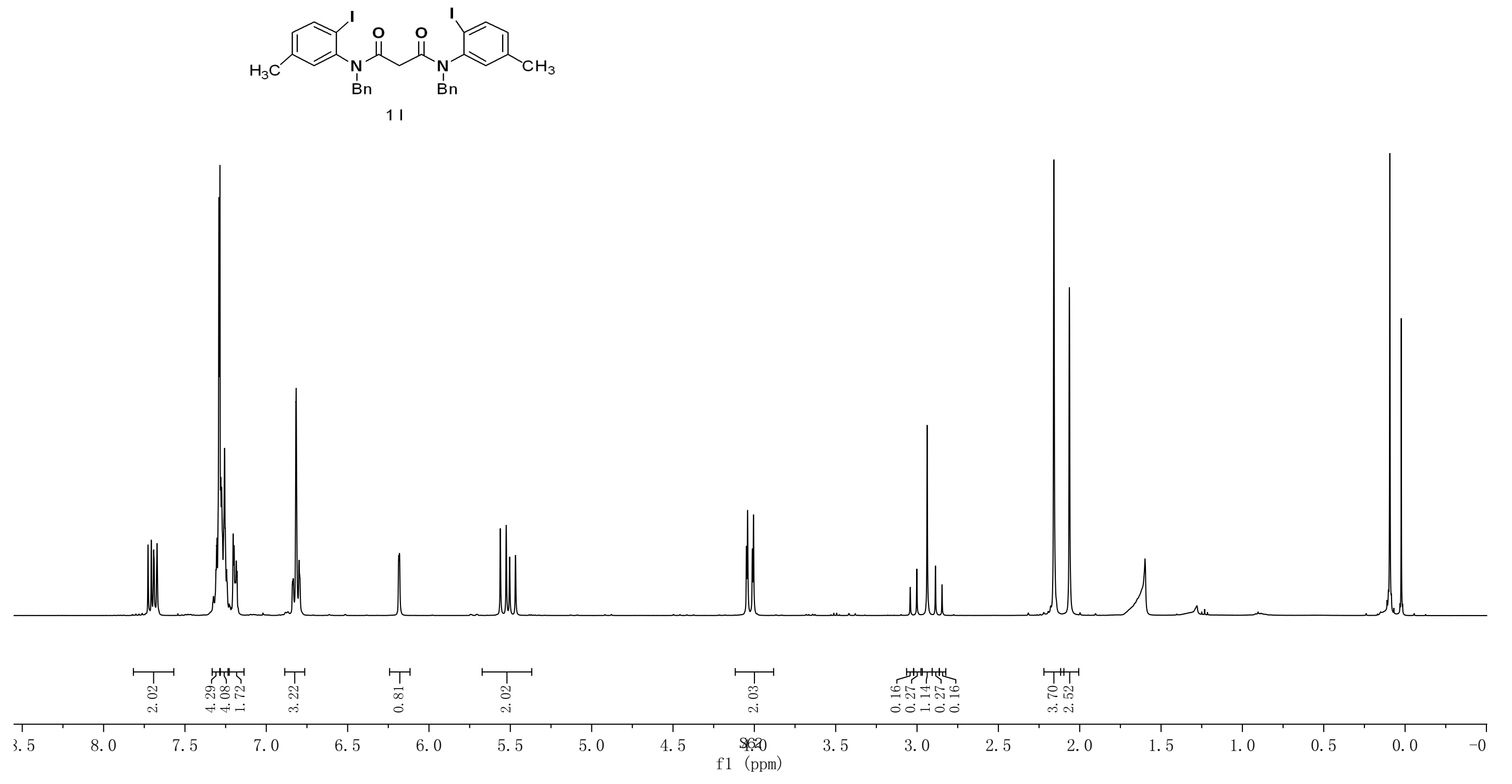

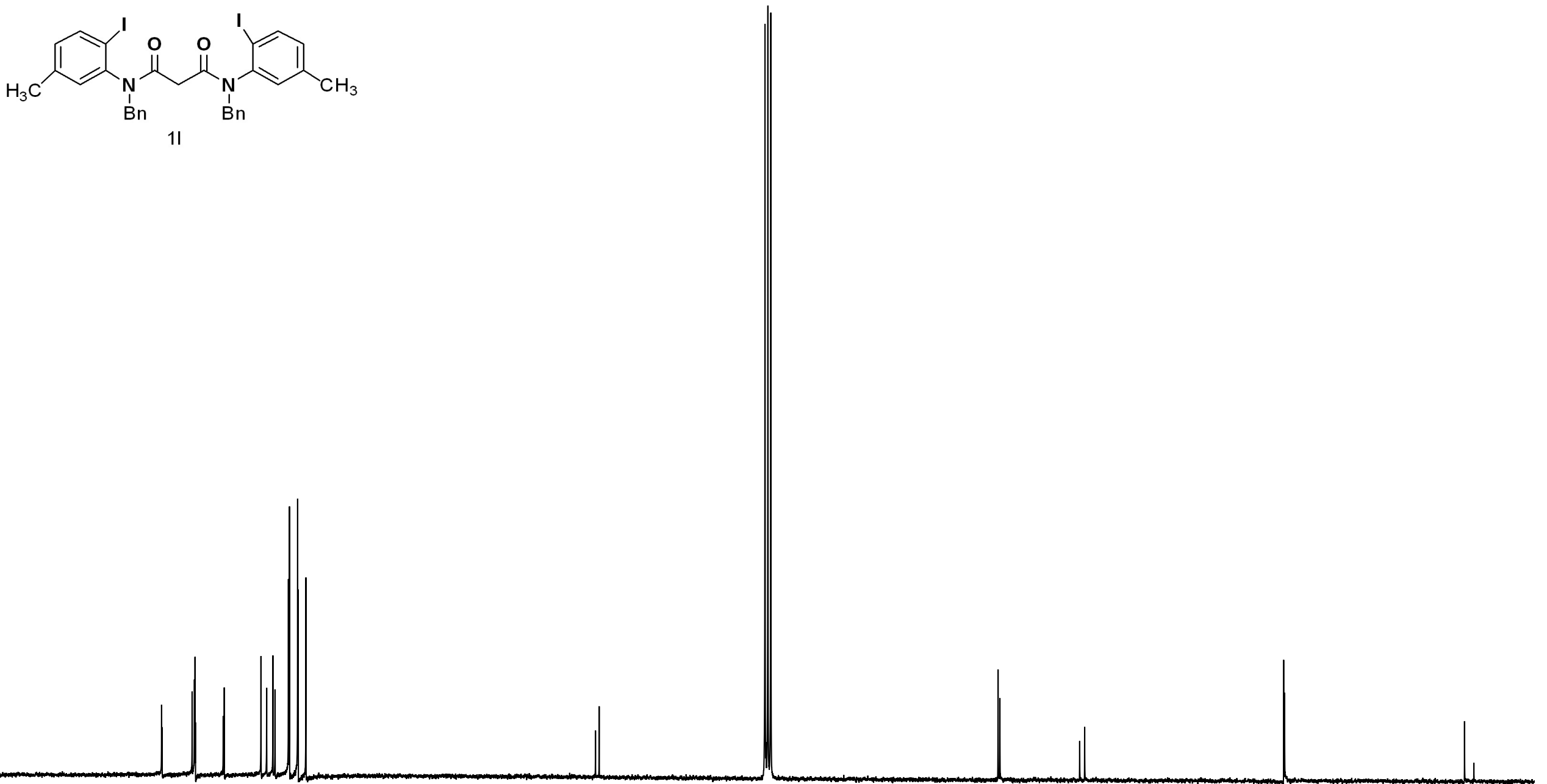

$$
\begin{gathered}
\mathrm{T} \\
180
\end{gathered}
$$



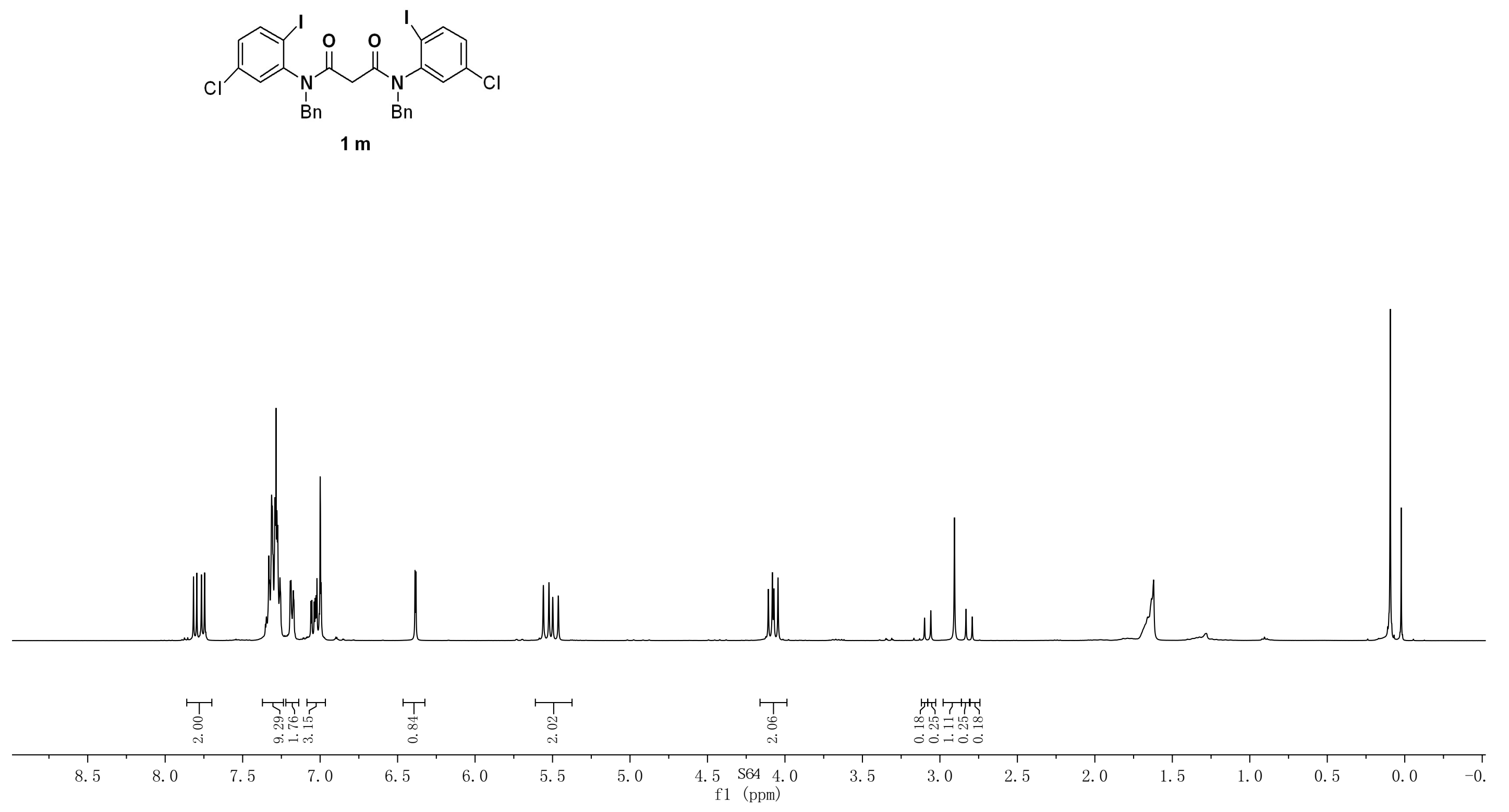

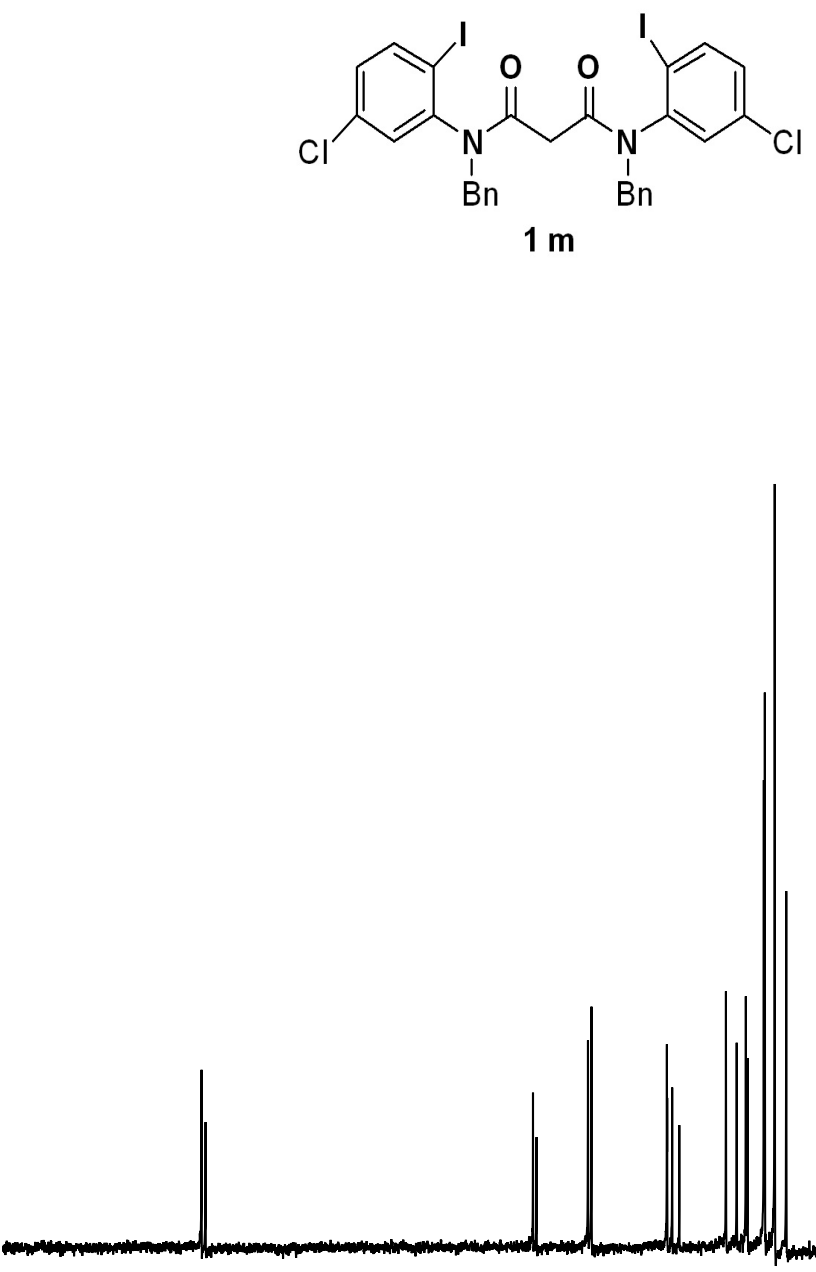


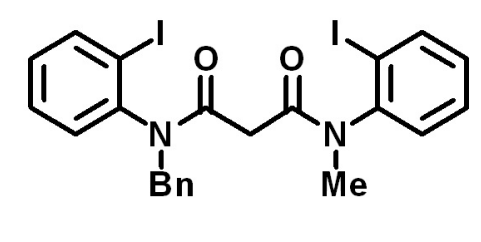

$3 a$

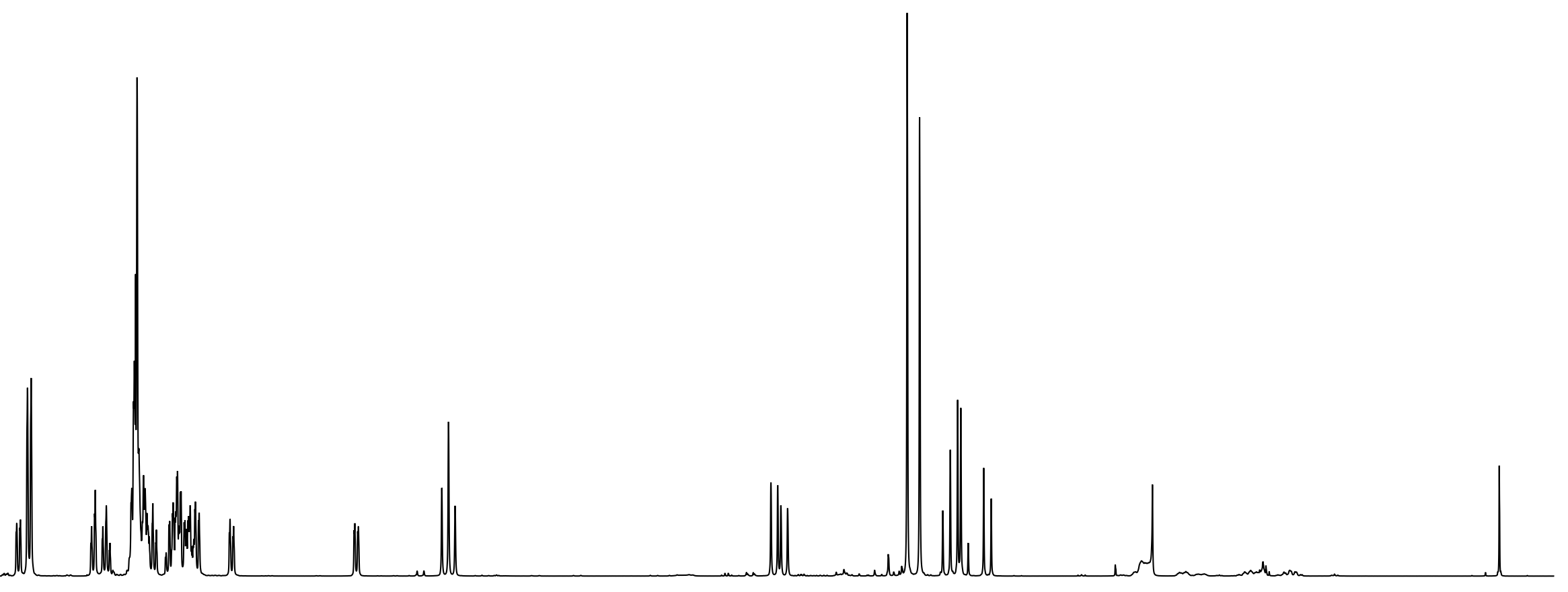

\begin{tabular}{|c|c|c|c|c|c|c|c|c|c|c|c|c|c|c|c|c|c|c|}
\hline & & & 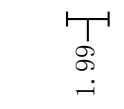 & 독 & |r & 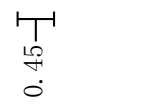 & \begin{tabular}{l}
$T^{1}$ \\
\multirow{2}{*}{} \\
0
\end{tabular} & 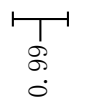 & & $\begin{array}{l}1 \\
\text { S. } \\
\vdots \\
0\end{array}$ & & 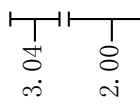 & & & & & & \\
\hline 9.5 & 9. 0 & 8.5 & 8.0 & 7.5 & 7. 0 & 6.5 & 6.0 & 5.5 & $\begin{array}{c}5.0 \quad \mathrm{~S} 66 \quad 4.5 \\
\text { f1 (ppm) }\end{array}$ & 4. 0 & 3.5 & 3.0 & 2.5 & 2.0 & 1.5 & 1.0 & 0.5 & 0.0 \\
\hline
\end{tabular}




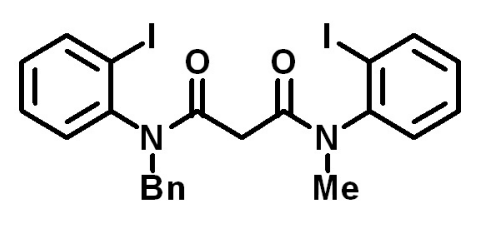

$3 a$
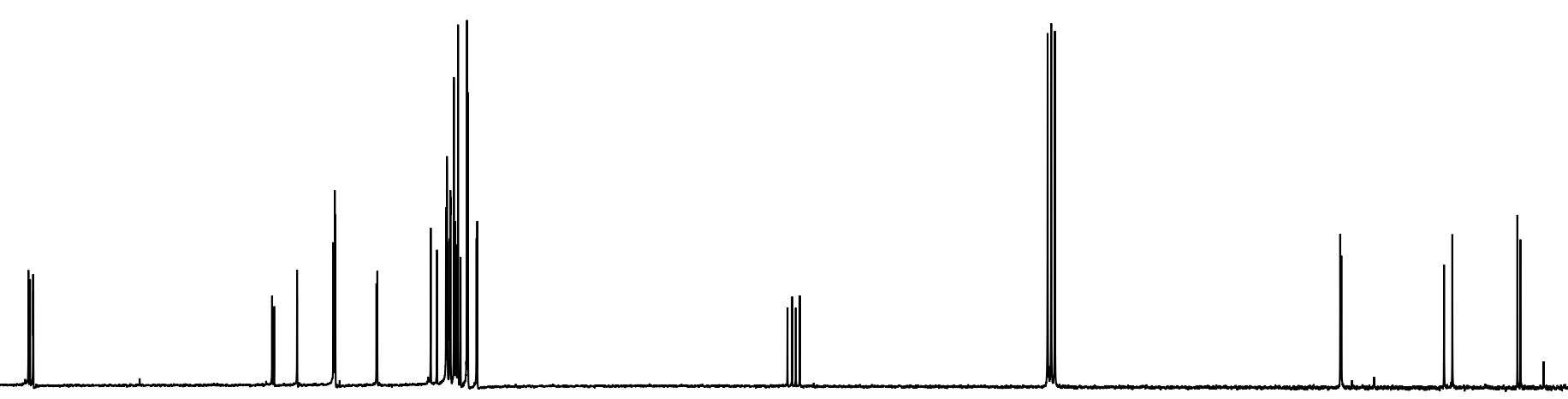

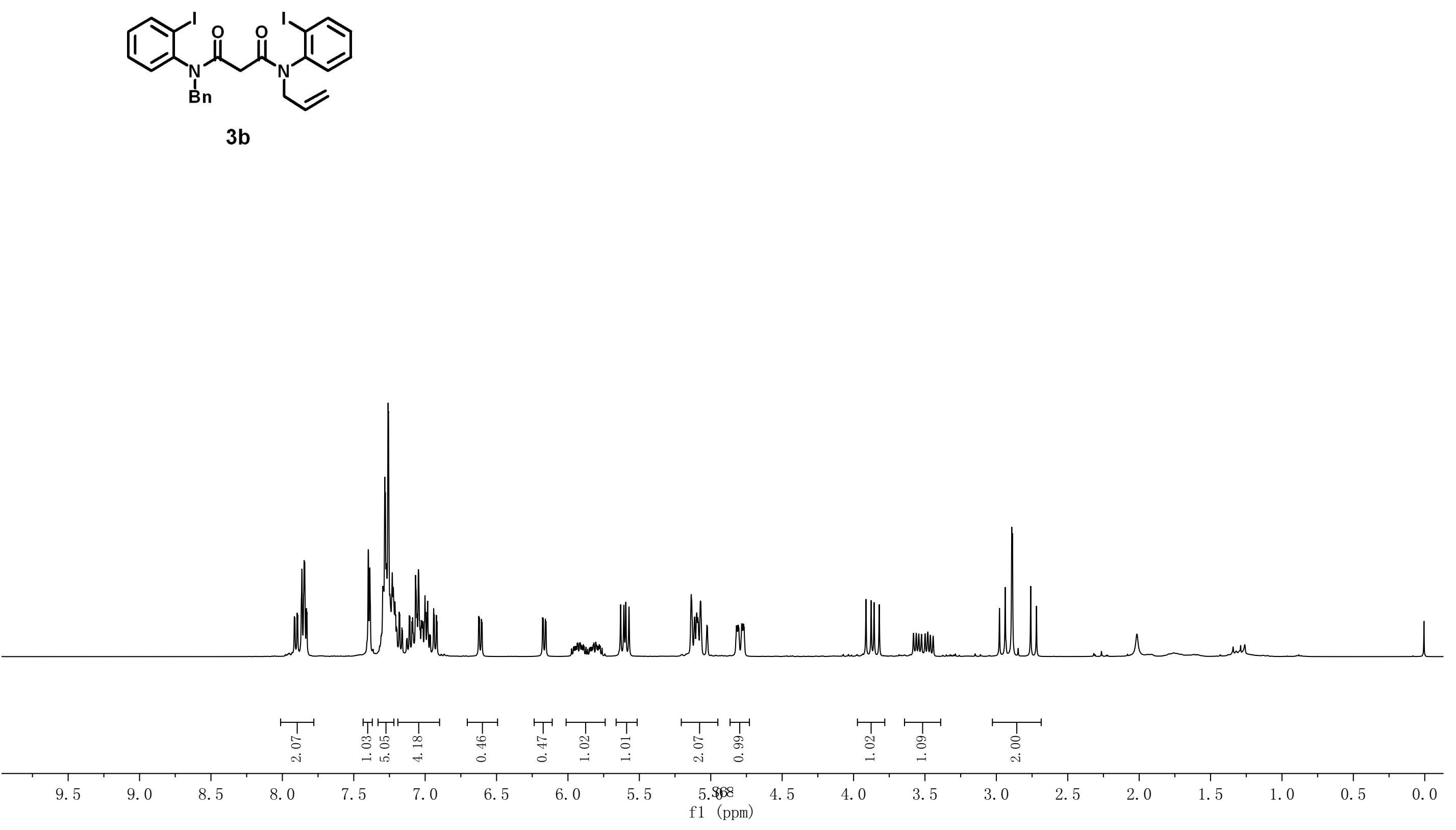


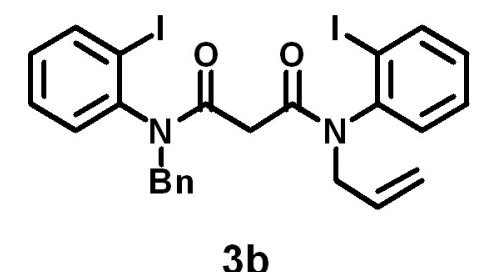

$3 b$
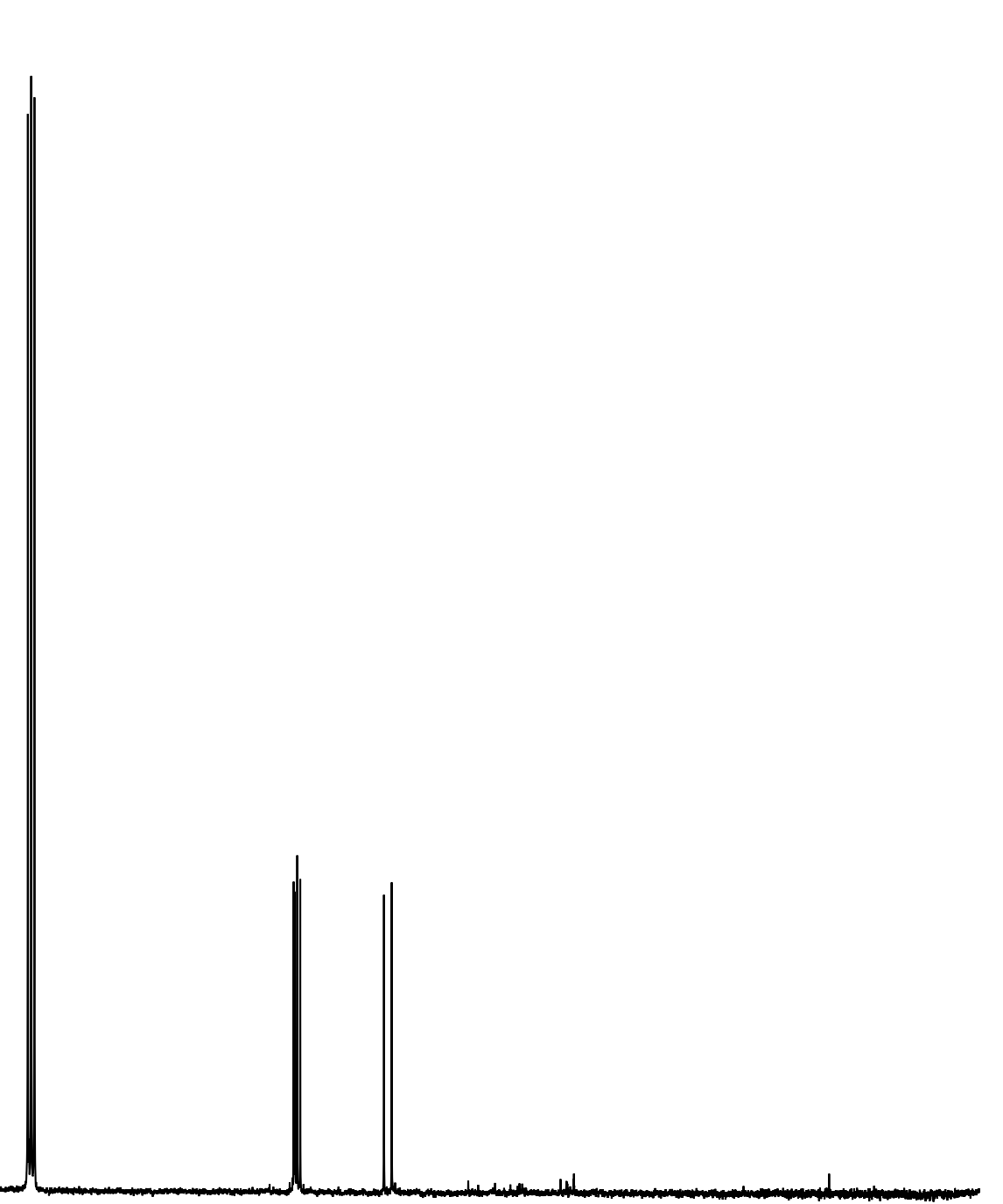

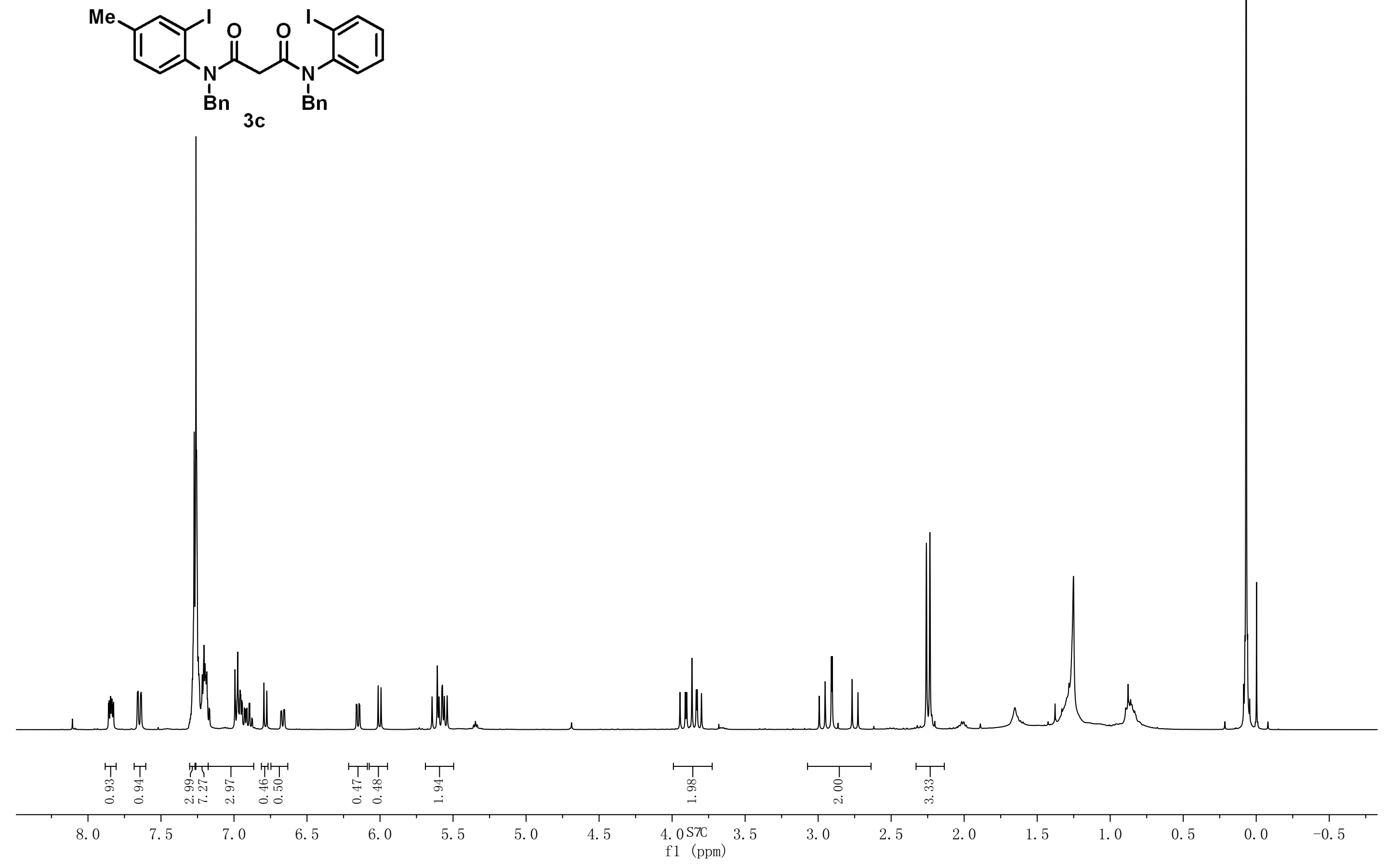

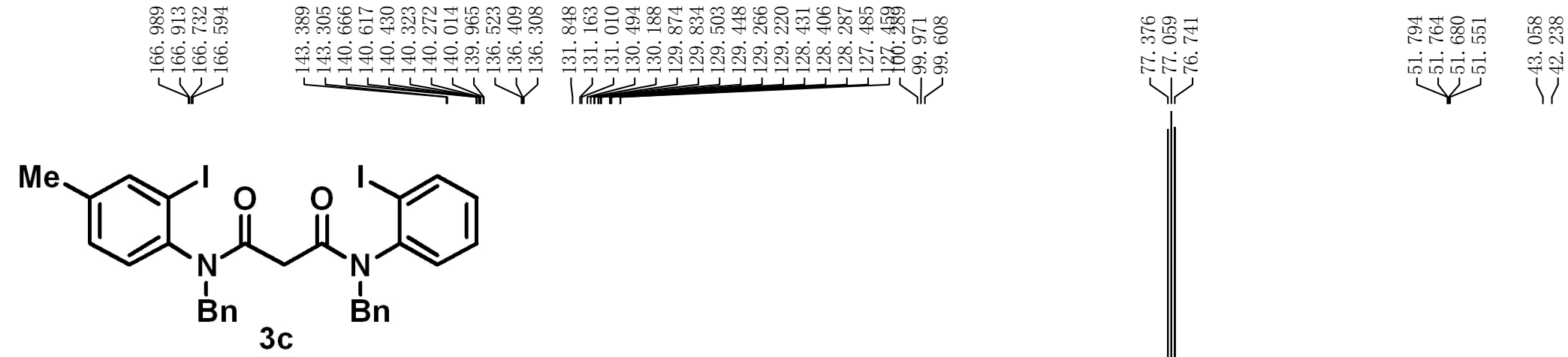

$$
\text { (1) }
$$



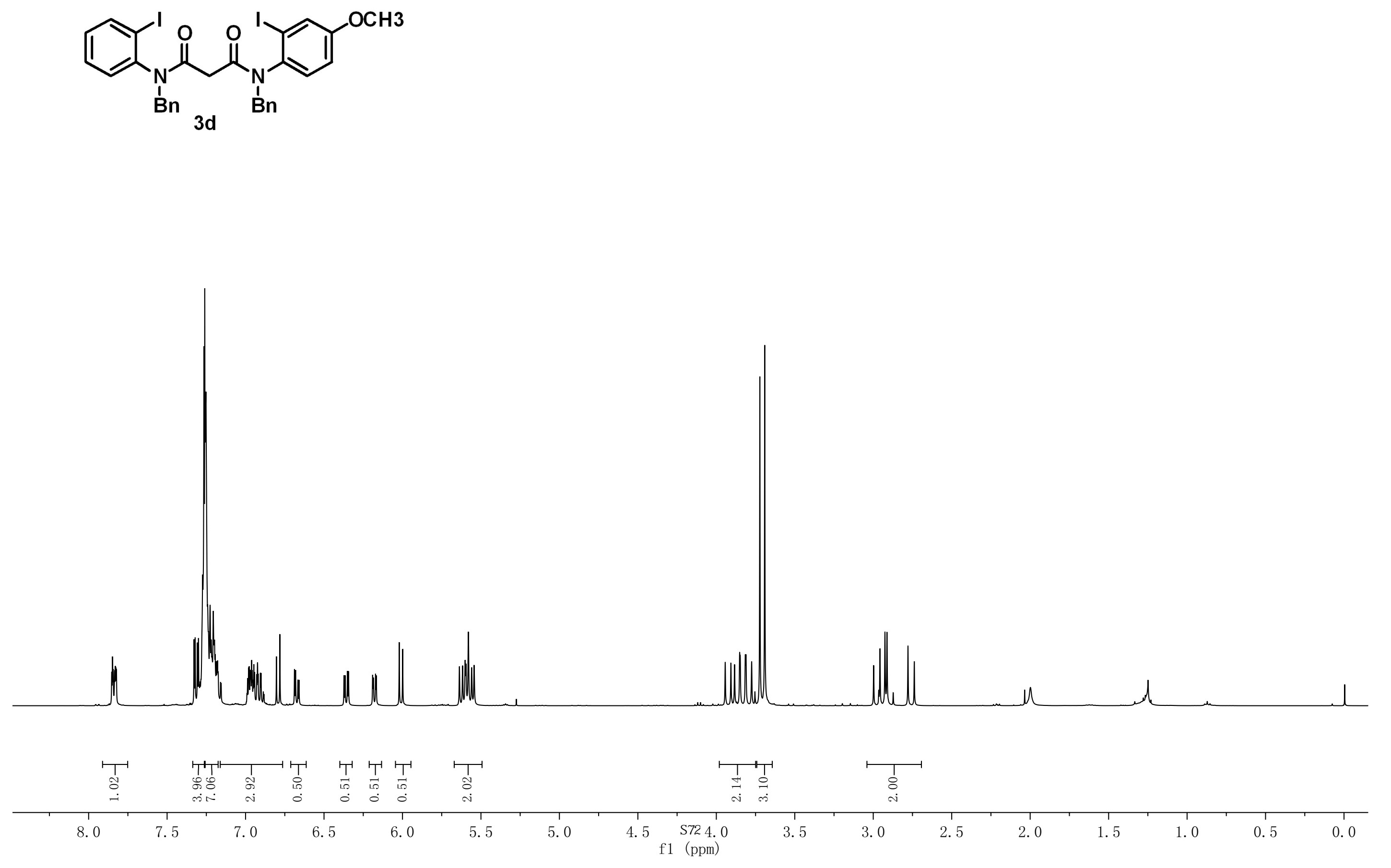

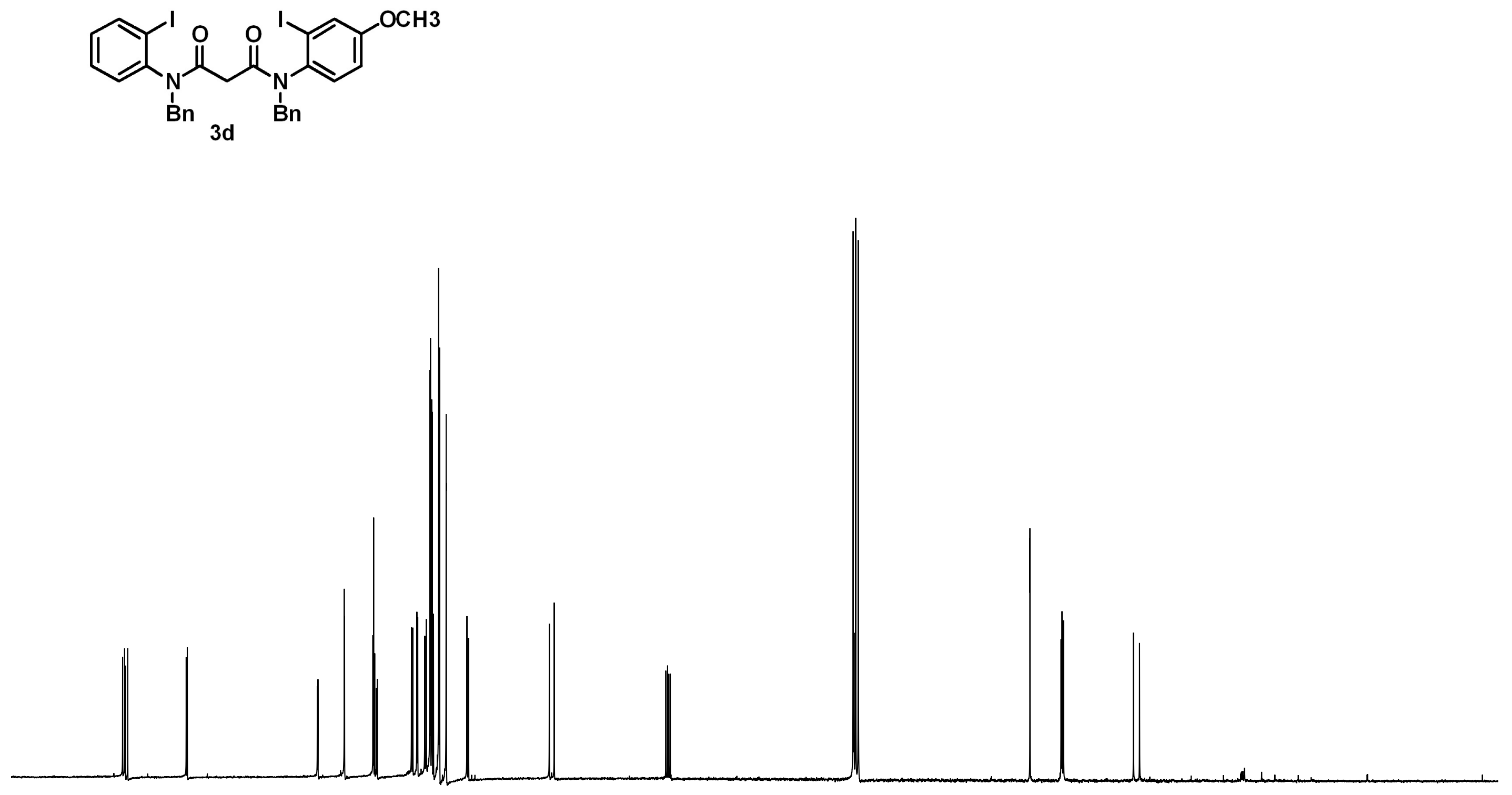

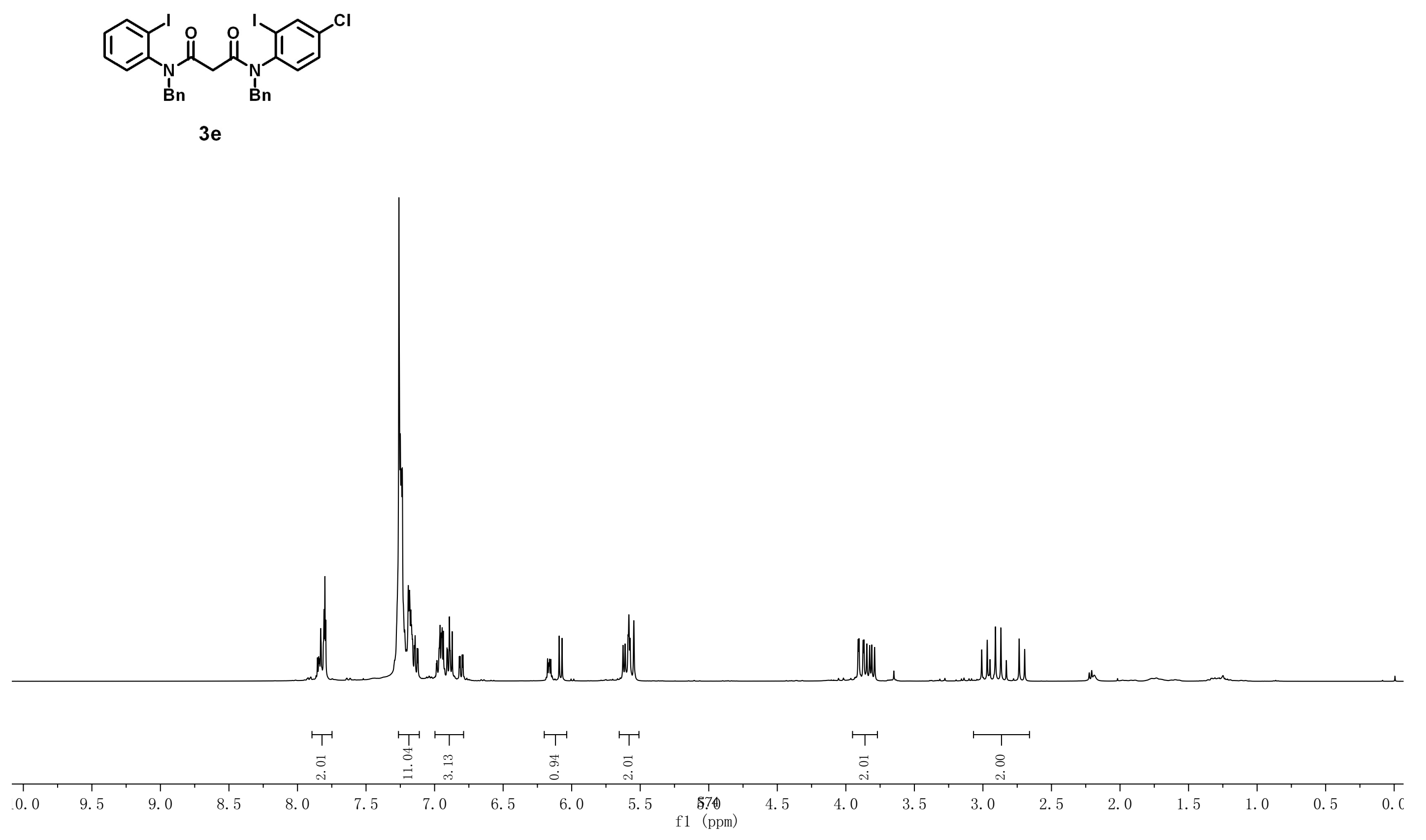

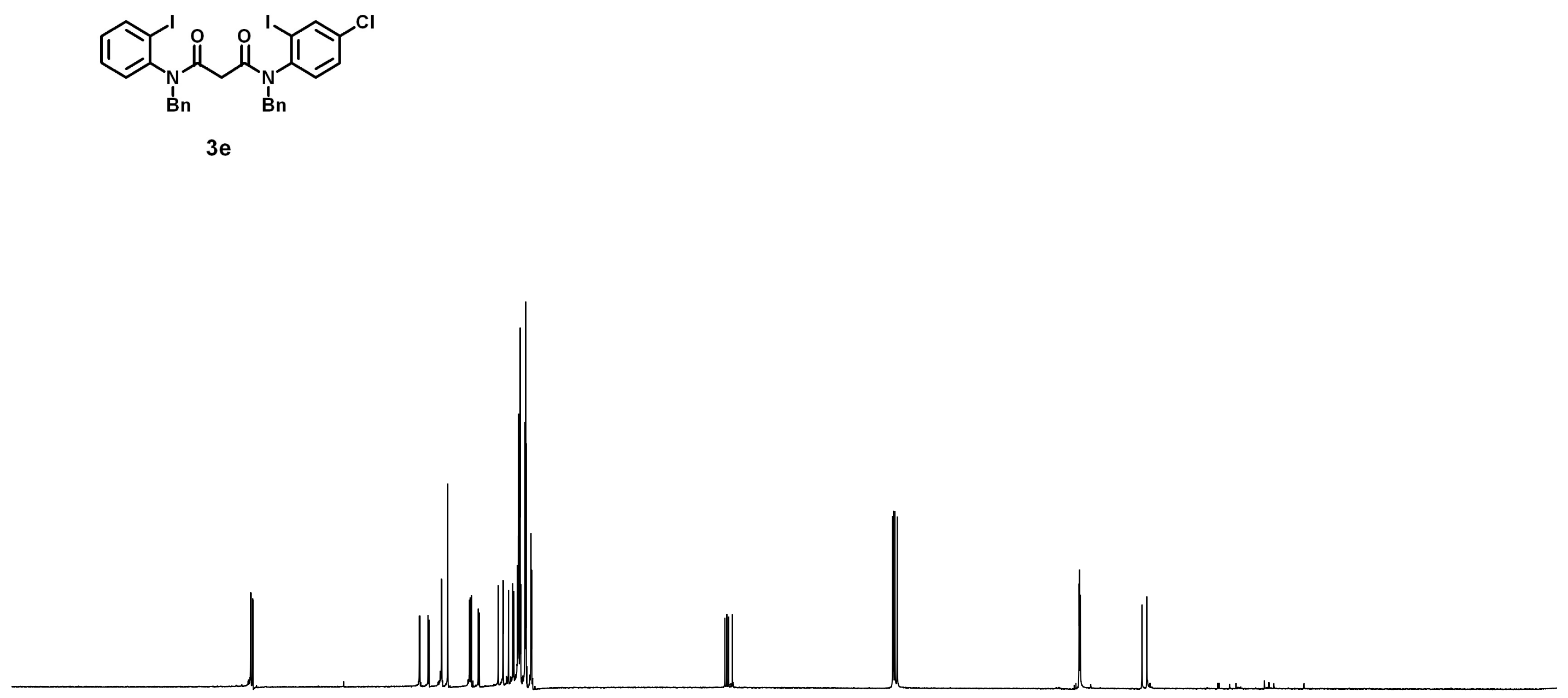

\begin{tabular}{|c|c|c|c|c|c|c|c|c|c|c|c|c|c|c|c|c|c|c|c|c|}
\hline 190 & 180 & 170 & 160 & 150 & 140 & 130 & 120 & 110 & & $\begin{array}{c}57590 \\
(\mathrm{ppm})\end{array}$ & 80 & 70 & 60 & 50 & $\begin{array}{l}1 \\
40\end{array}$ & 30 & 20 & 10 & 0 & -10 \\
\hline
\end{tabular}



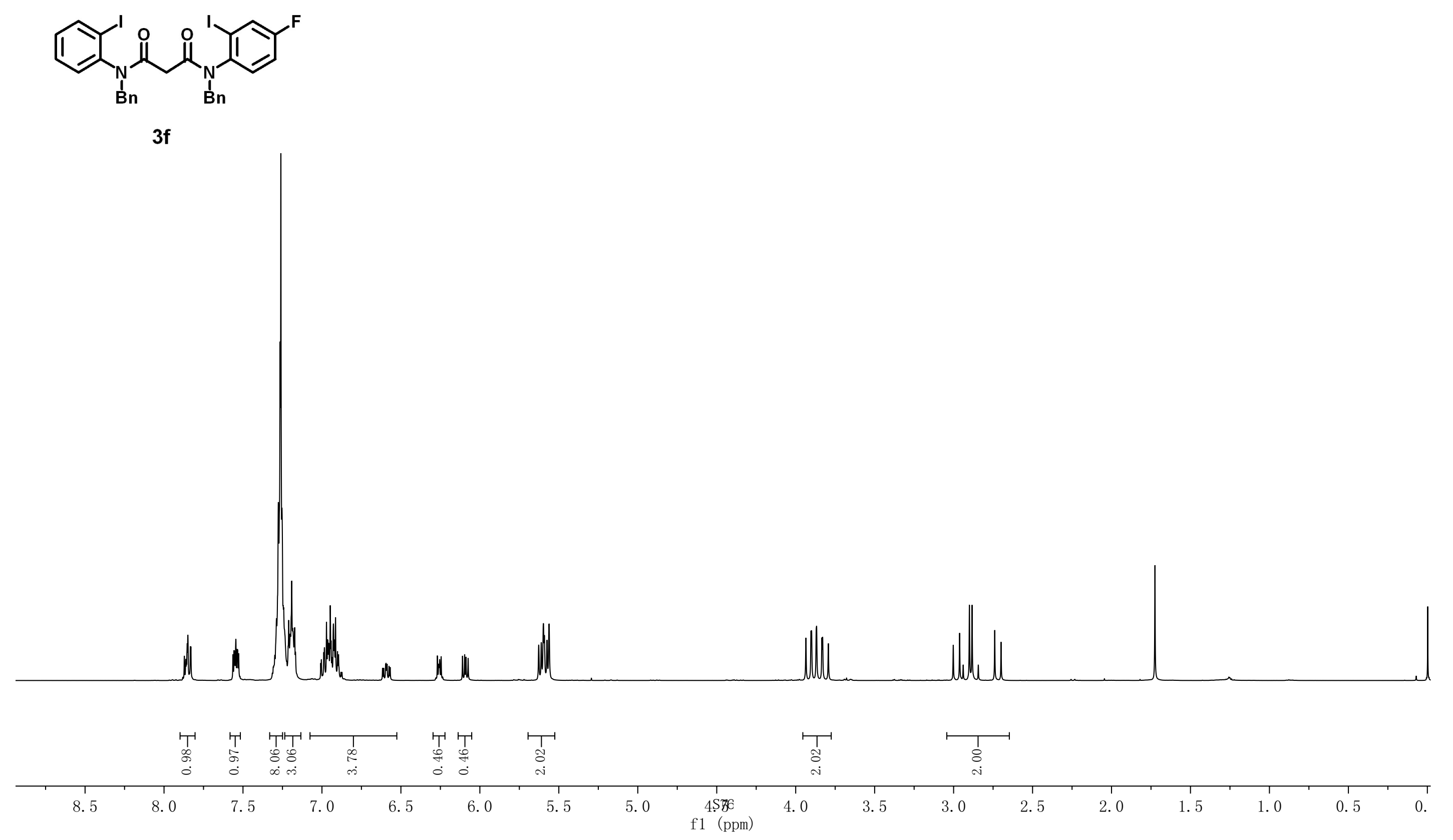


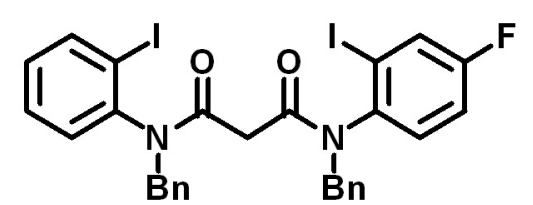

$3 f$

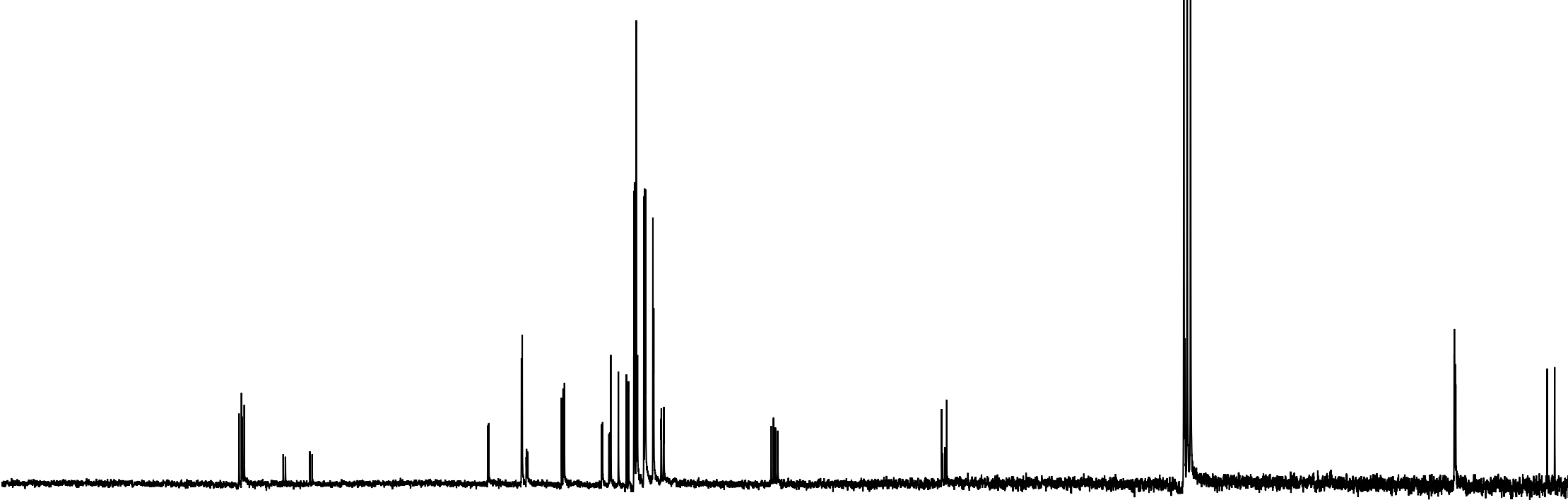

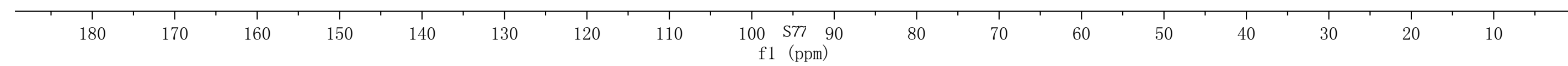




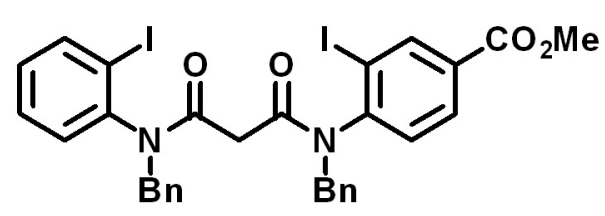

$3 g$

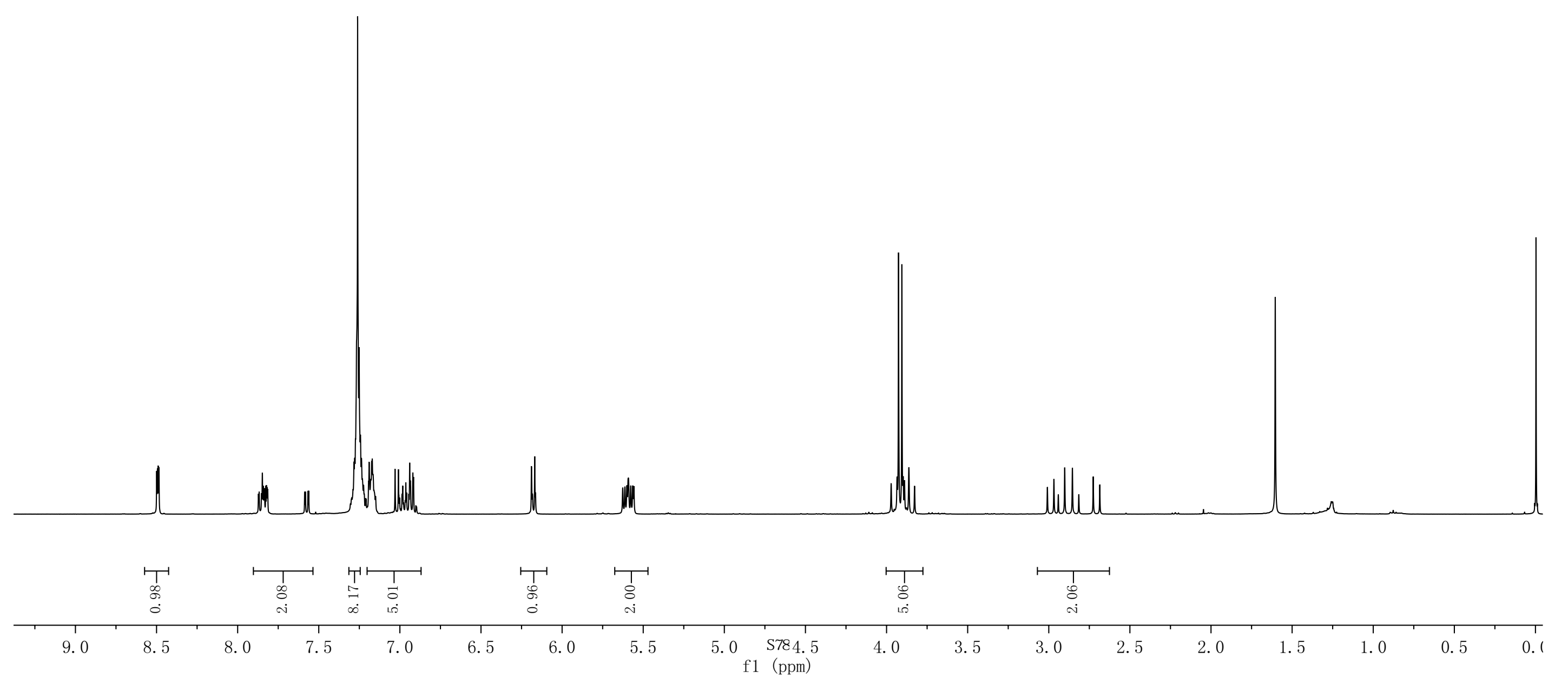




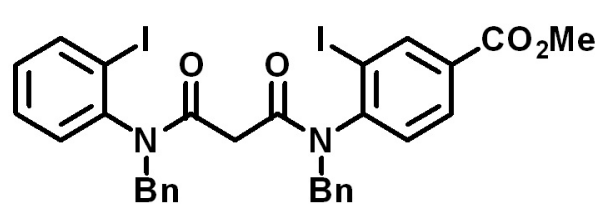

$3 g$

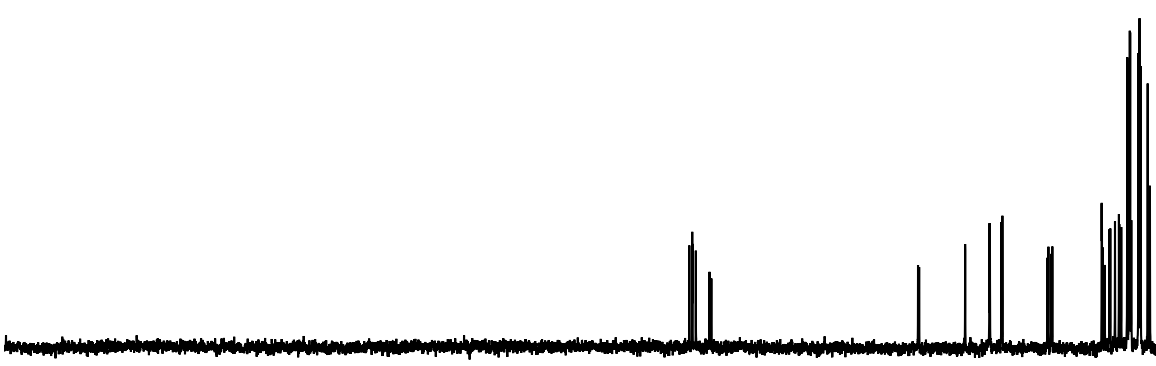




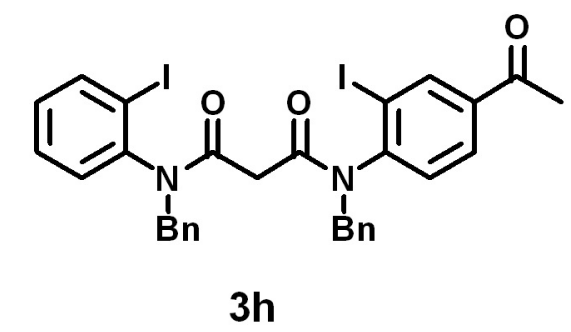

$3 h$
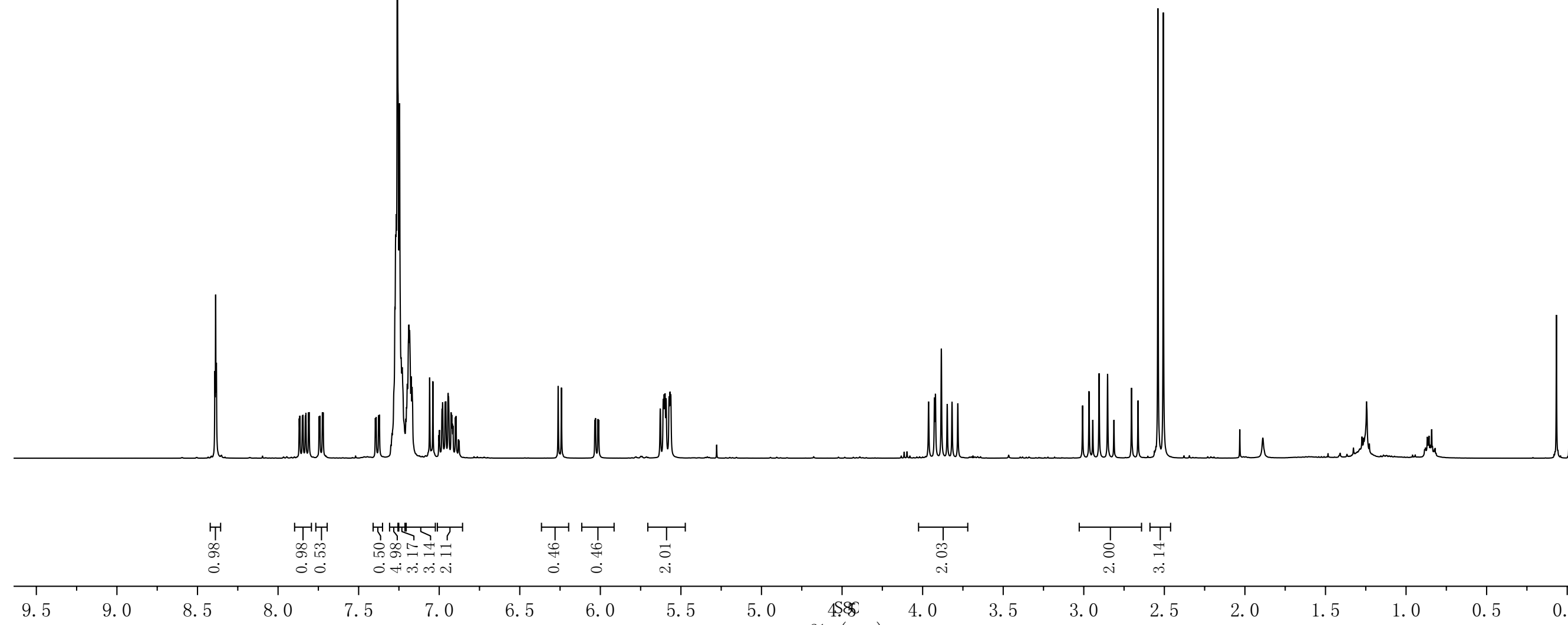

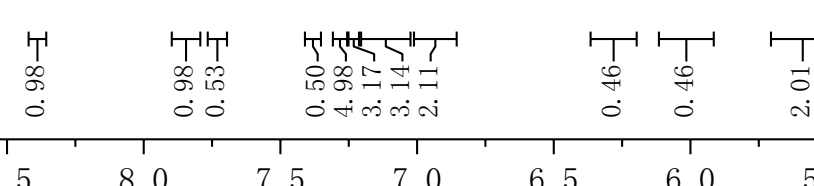

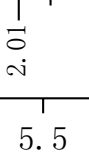


<smiles>[Mg][Mg]</smiles>

$3 h$

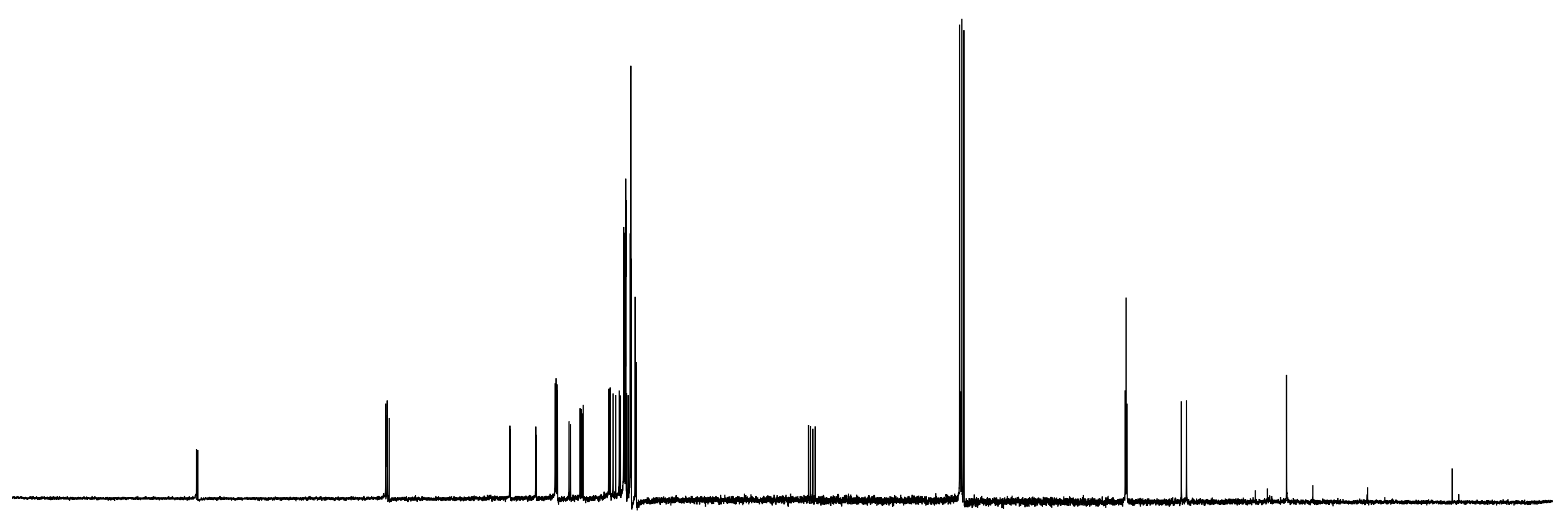

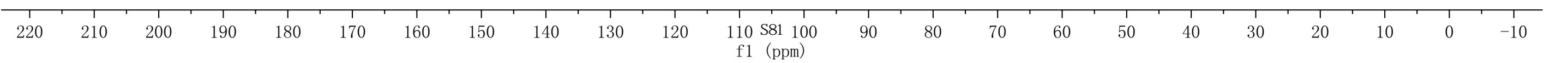



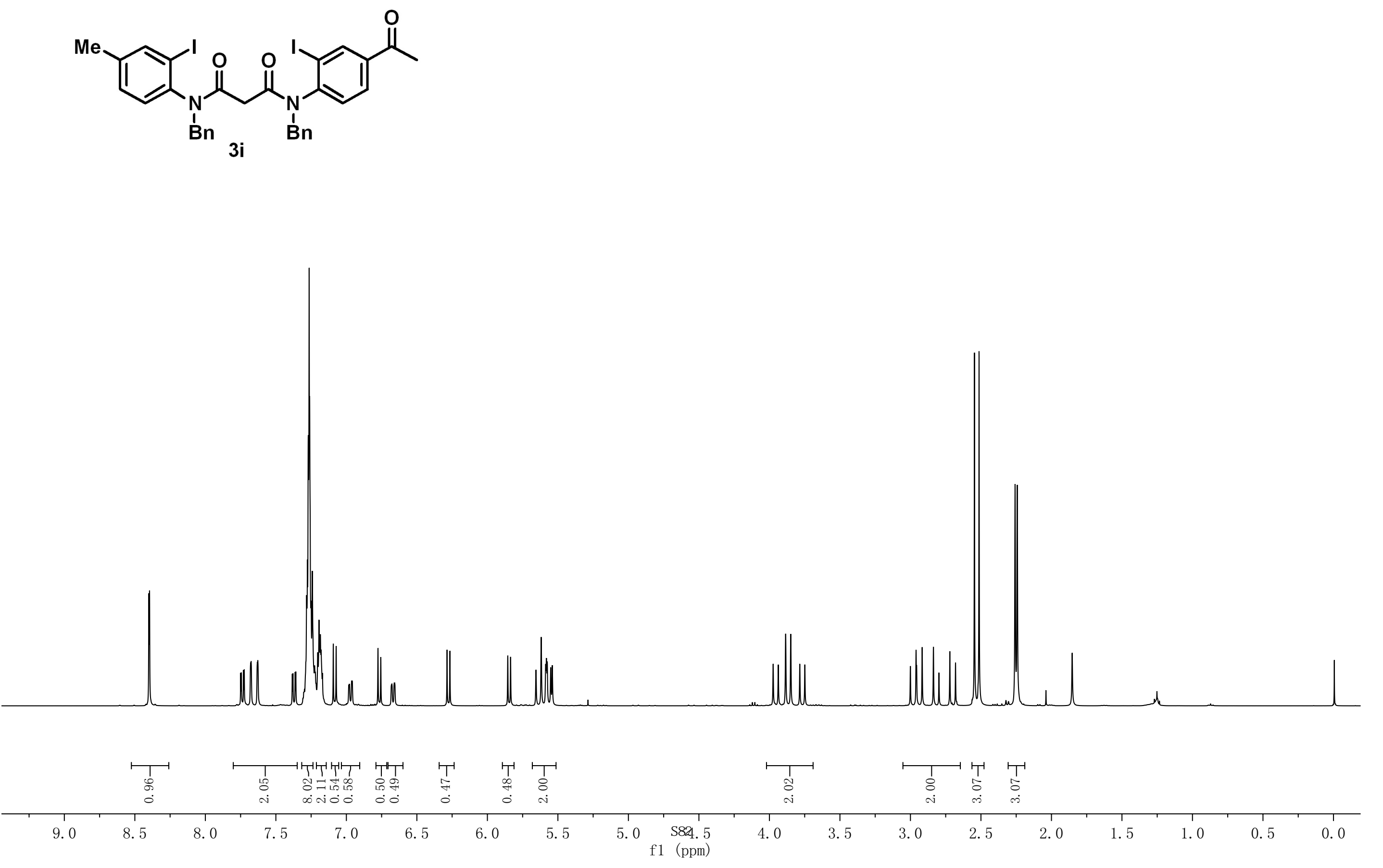
<smiles>CC(=O)c1ccc(N(Cc2ccccc2)C(=O)CC(=O)N(Cc2ccccc2)c2ccc(C)cc2I)c(I)c1</smiles>

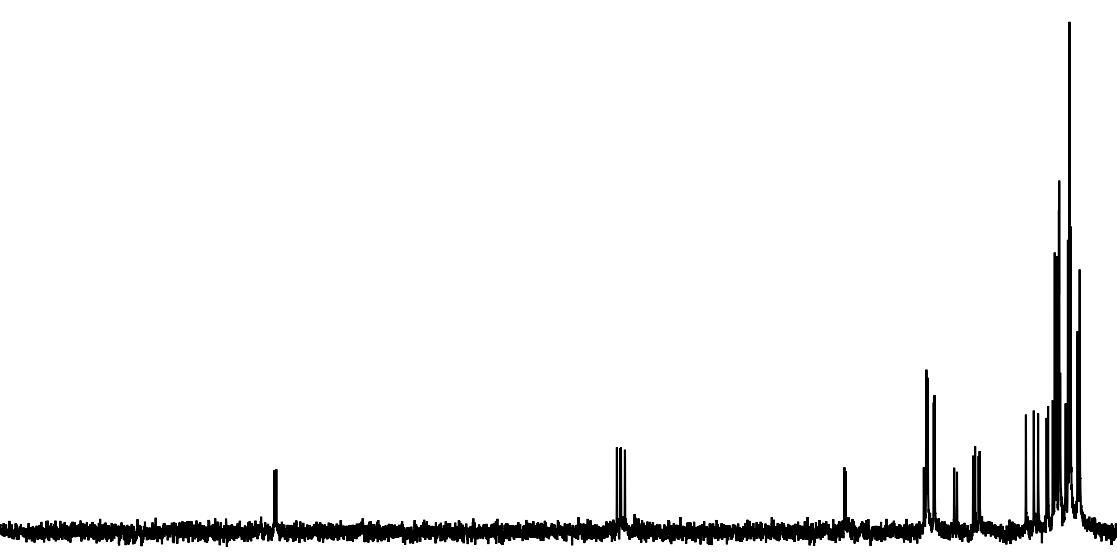




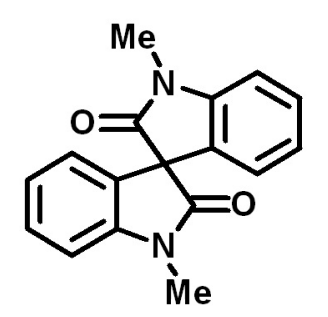

$2 a$
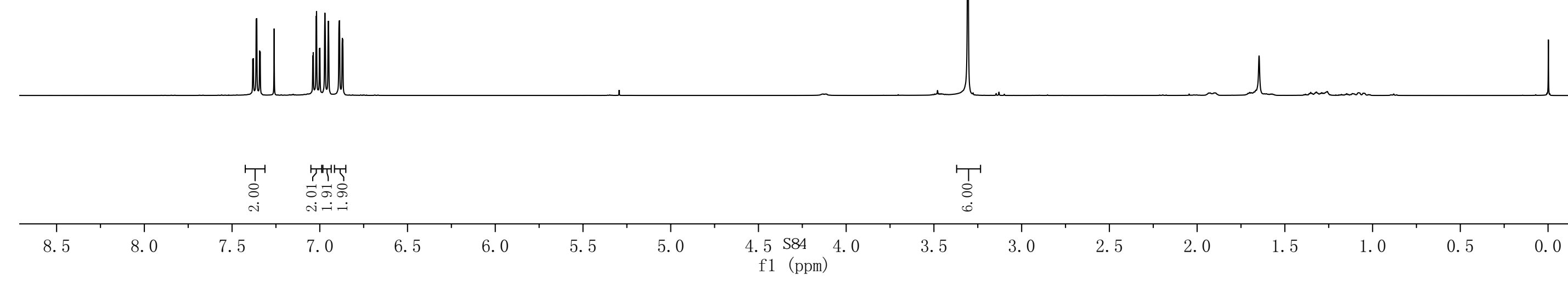


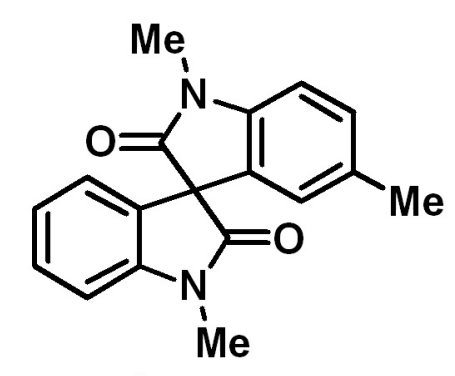

$2 a$

$$
22
$$

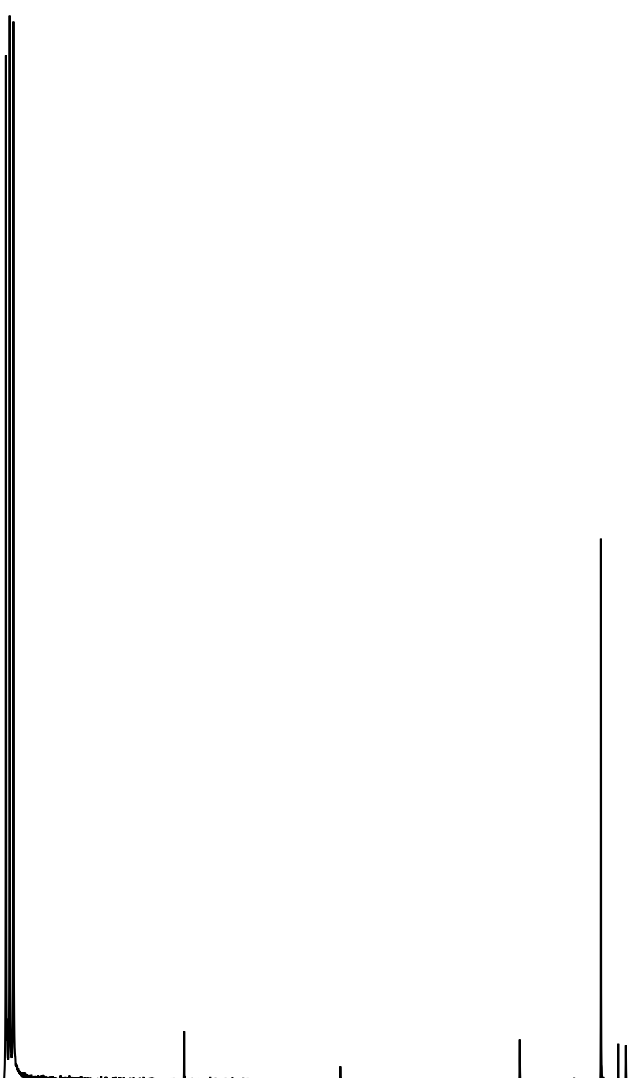




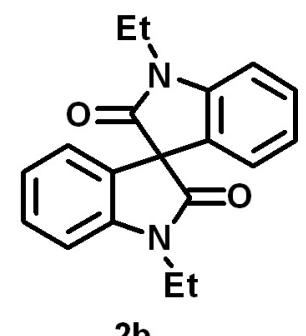

$2 b$

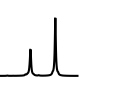

\begin{tabular}{|c|c|c|c|c|c|c|c|c|c|c|c|c|c|c|c|c|}
\hline & & $\begin{array}{l}\mathrm{T} \\
8 \\
\mathrm{i}\end{array}$ & 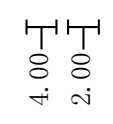 & & & & & & & & & & & & & \\
\hline .5 & 8.0 & 7.5 & 7.0 & 6.5 & 6.0 & 5.5 & 5.0 & 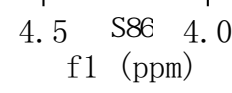 & 3.5 & 3. 0 & 2.5 & 2.0 & 1.5 & 1.0 & 0.5 & 0.0 \\
\hline
\end{tabular}


CC20181120-2

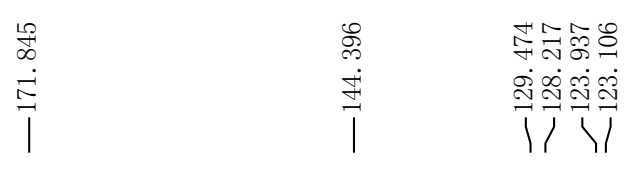

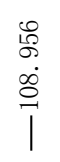

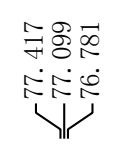

i

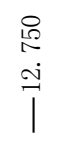

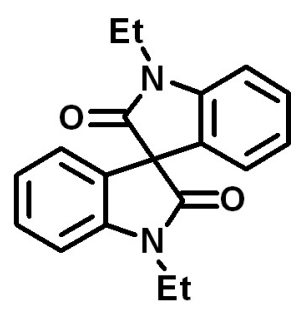

$2 b$
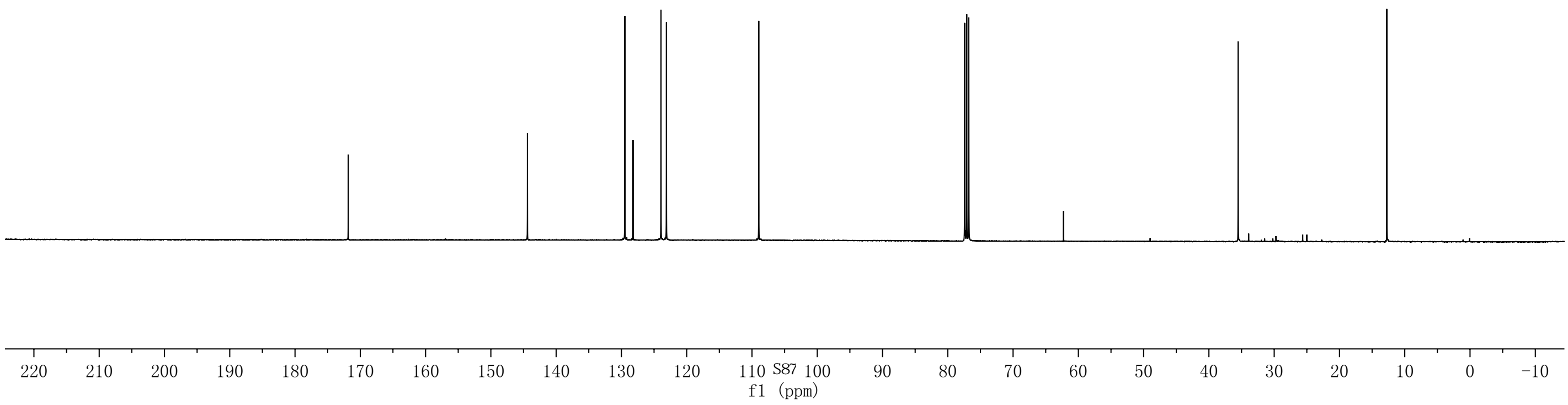

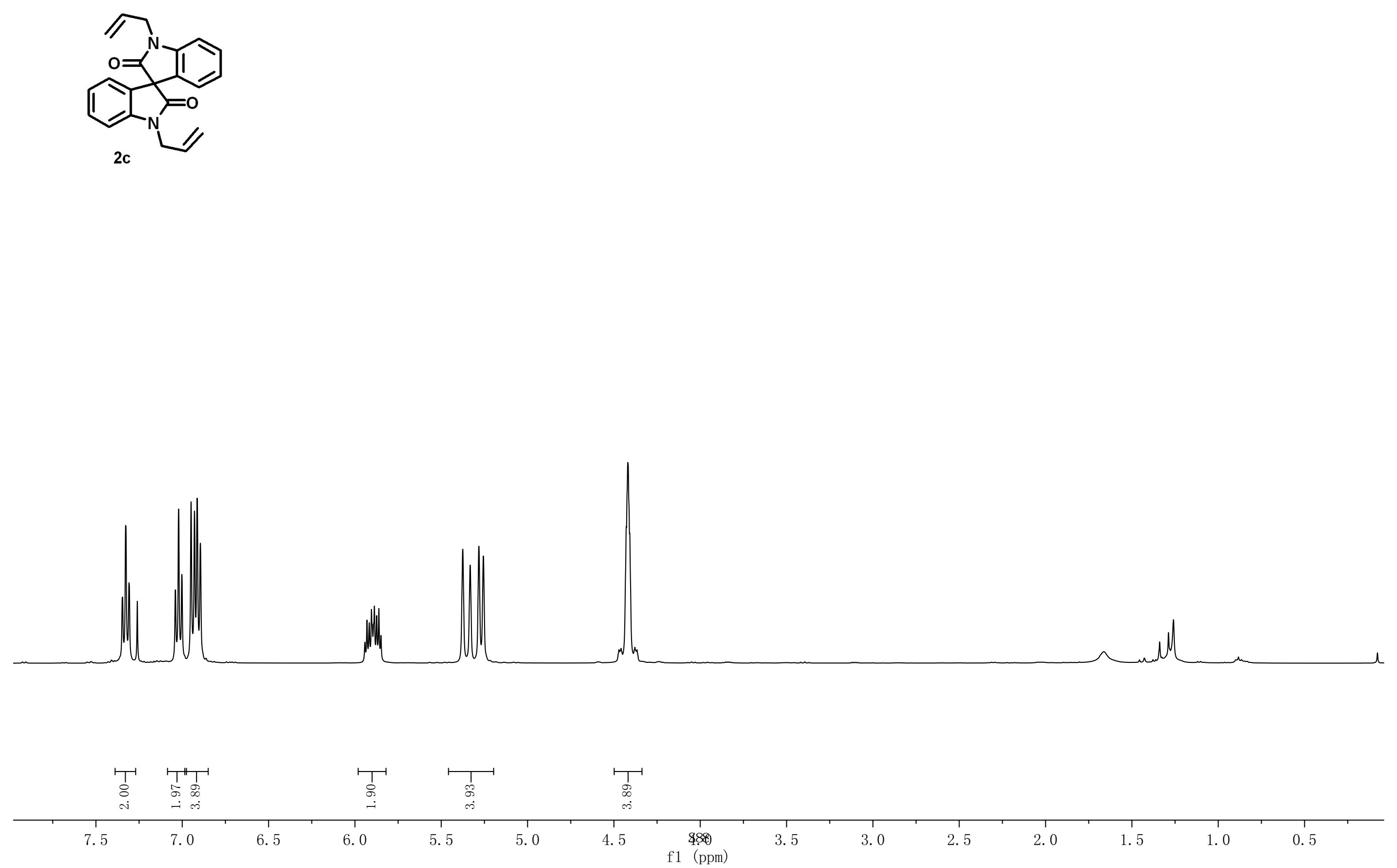
<smiles>C=CCN1C(=O)C2(C(=O)N(CC=C)c3ccccc32)c2ccccc21</smiles>

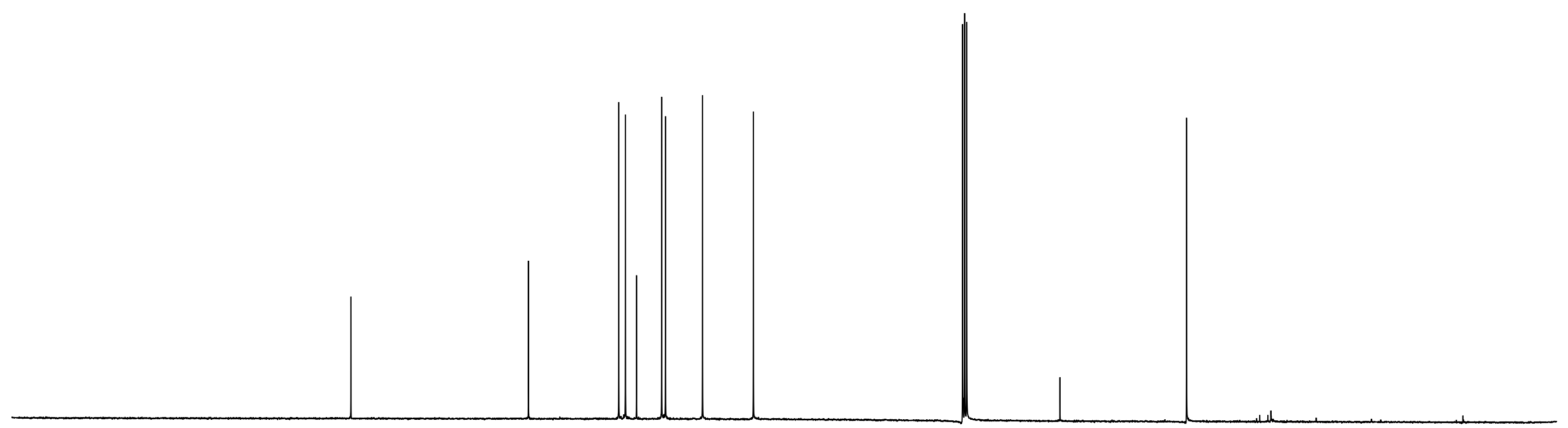

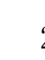




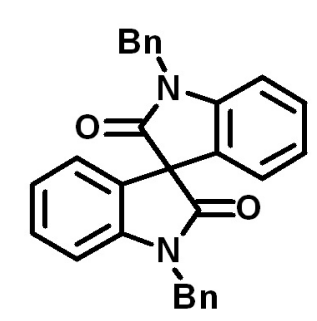

2d
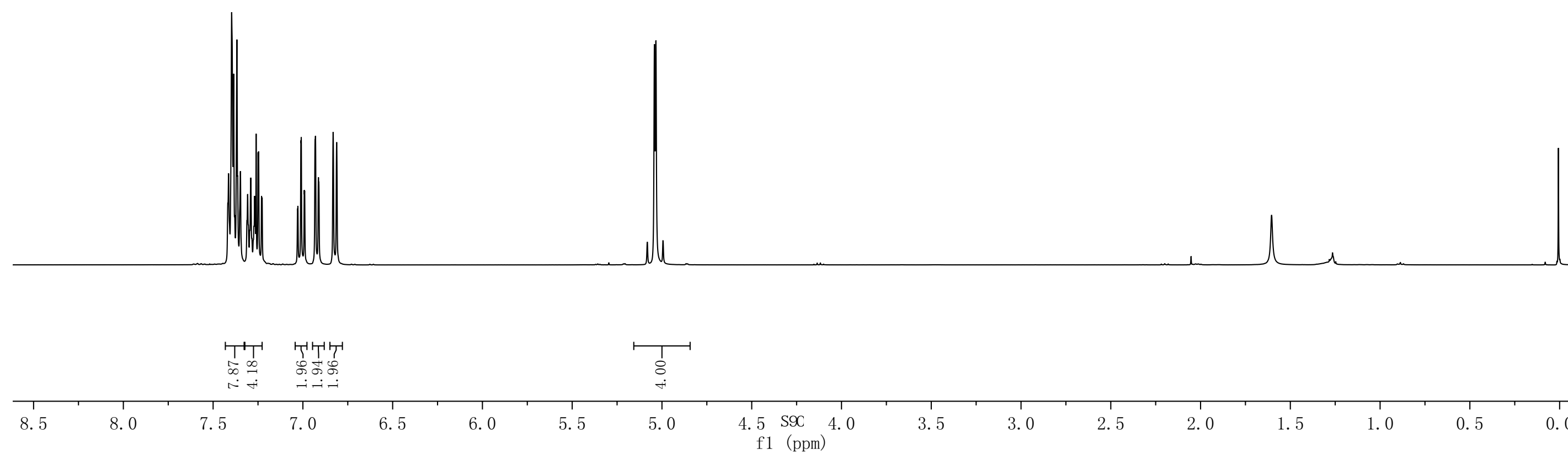
<smiles>O=C1N(Cc2ccccc2)c2ccccc2C12C(=O)N(Cc1ccccc1)c1ccccc12</smiles> 


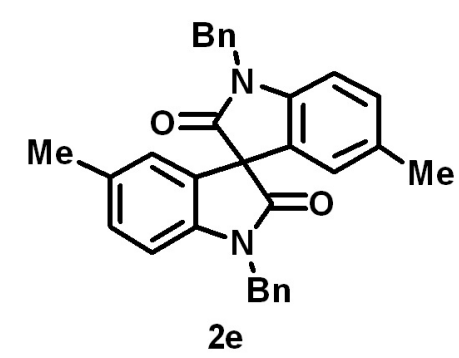

$2 \mathrm{e}$
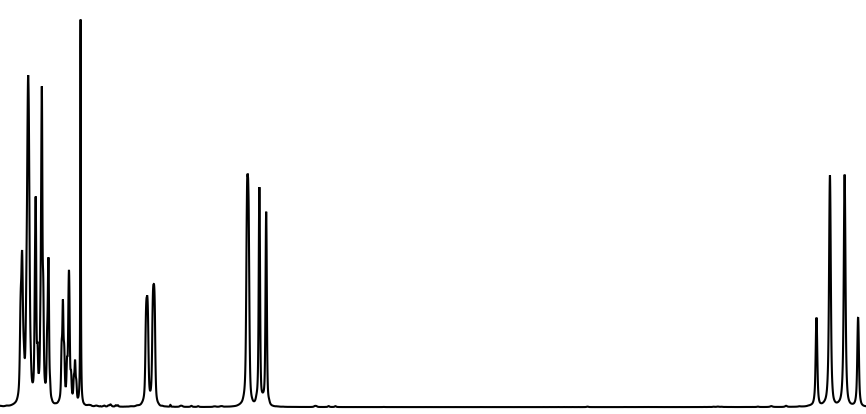

M

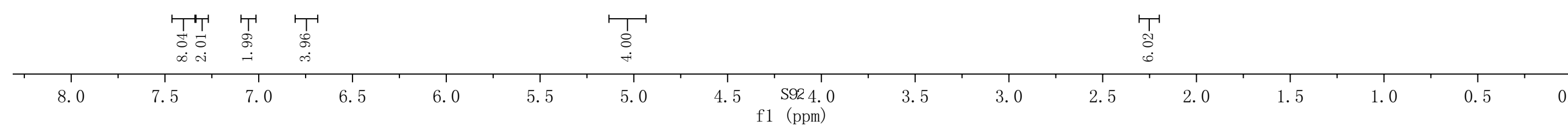


CC20181118-2C
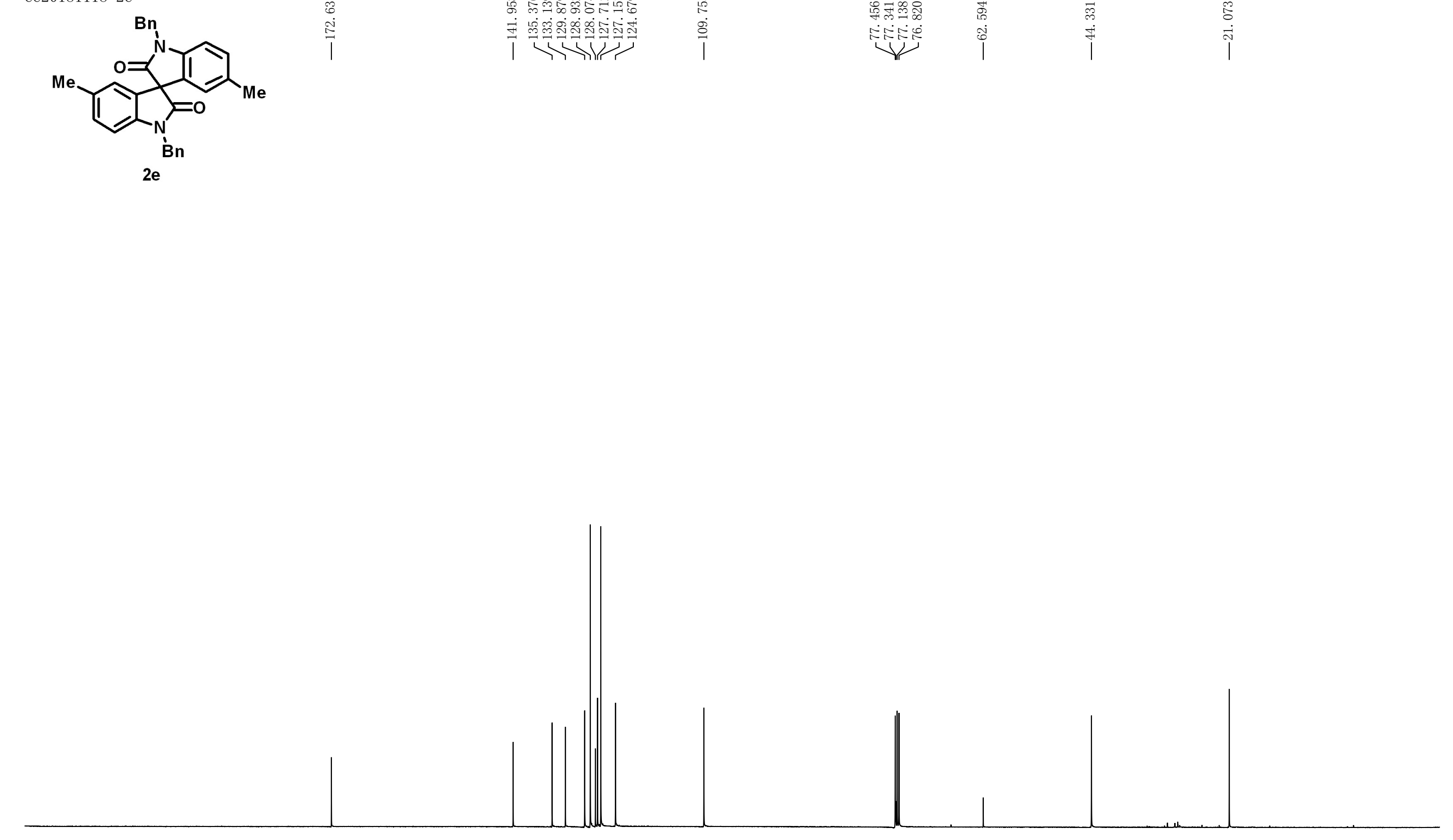

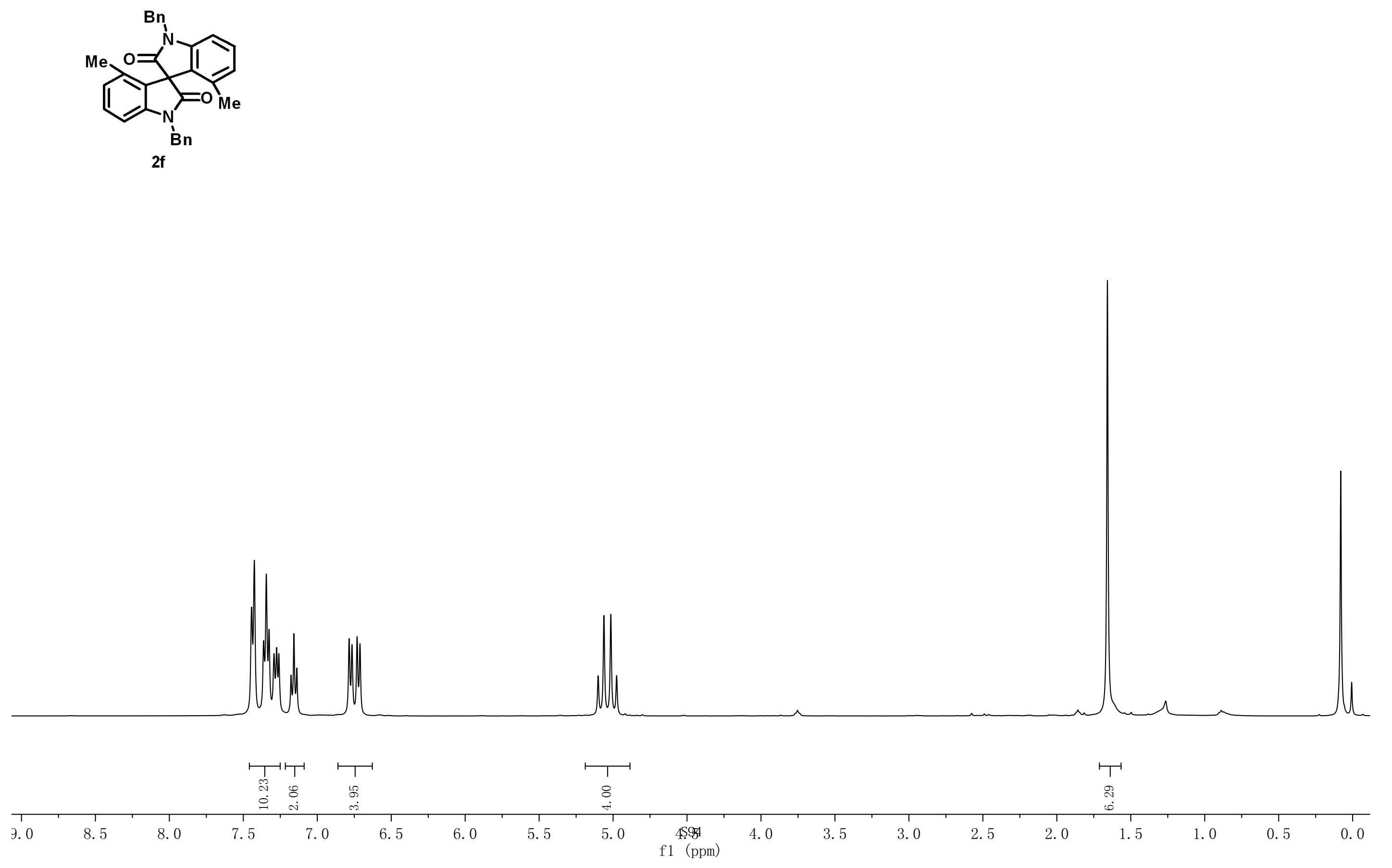


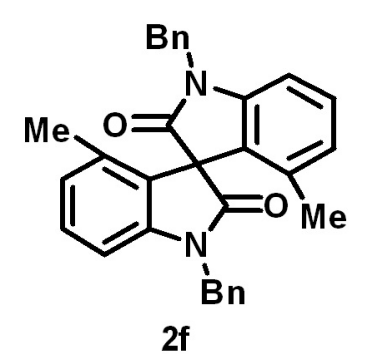

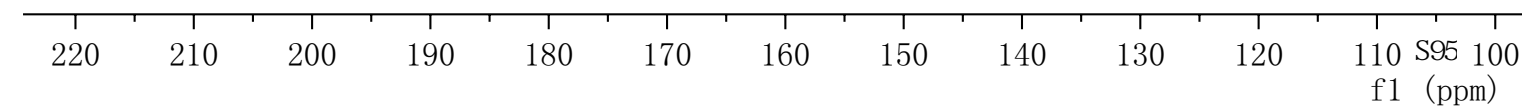


LT201802030. 2

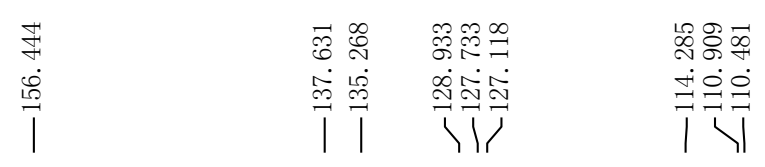

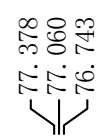

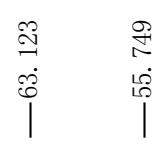

$\infty$
$\infty$
$\infty$
$\stackrel{+}{+}$

OMe

$2 g$
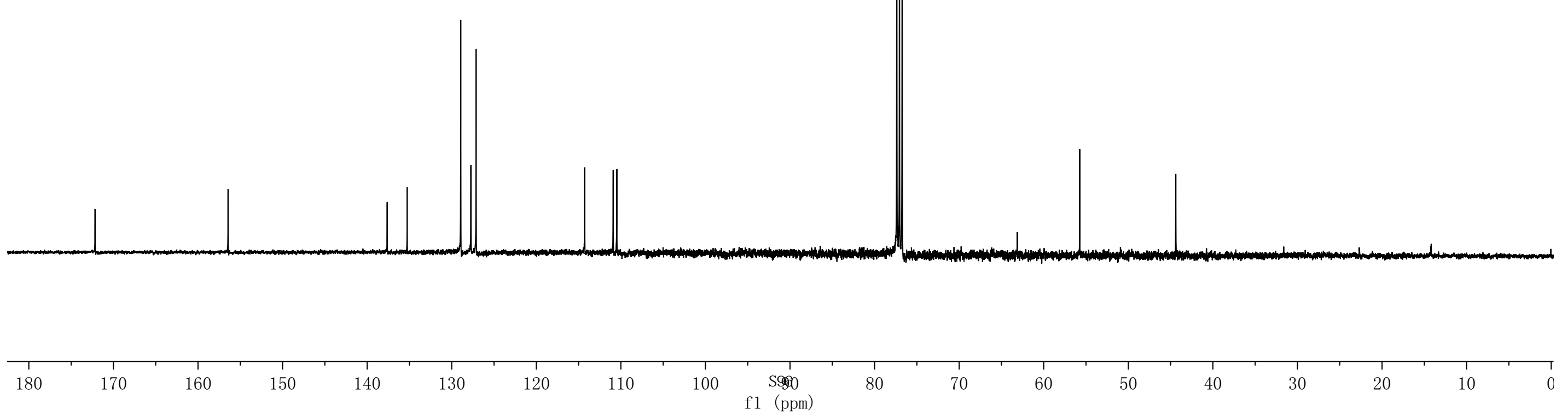


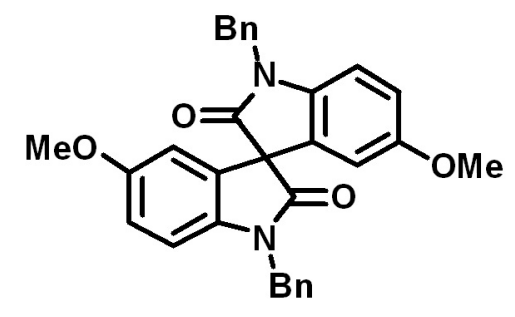

$2 g$

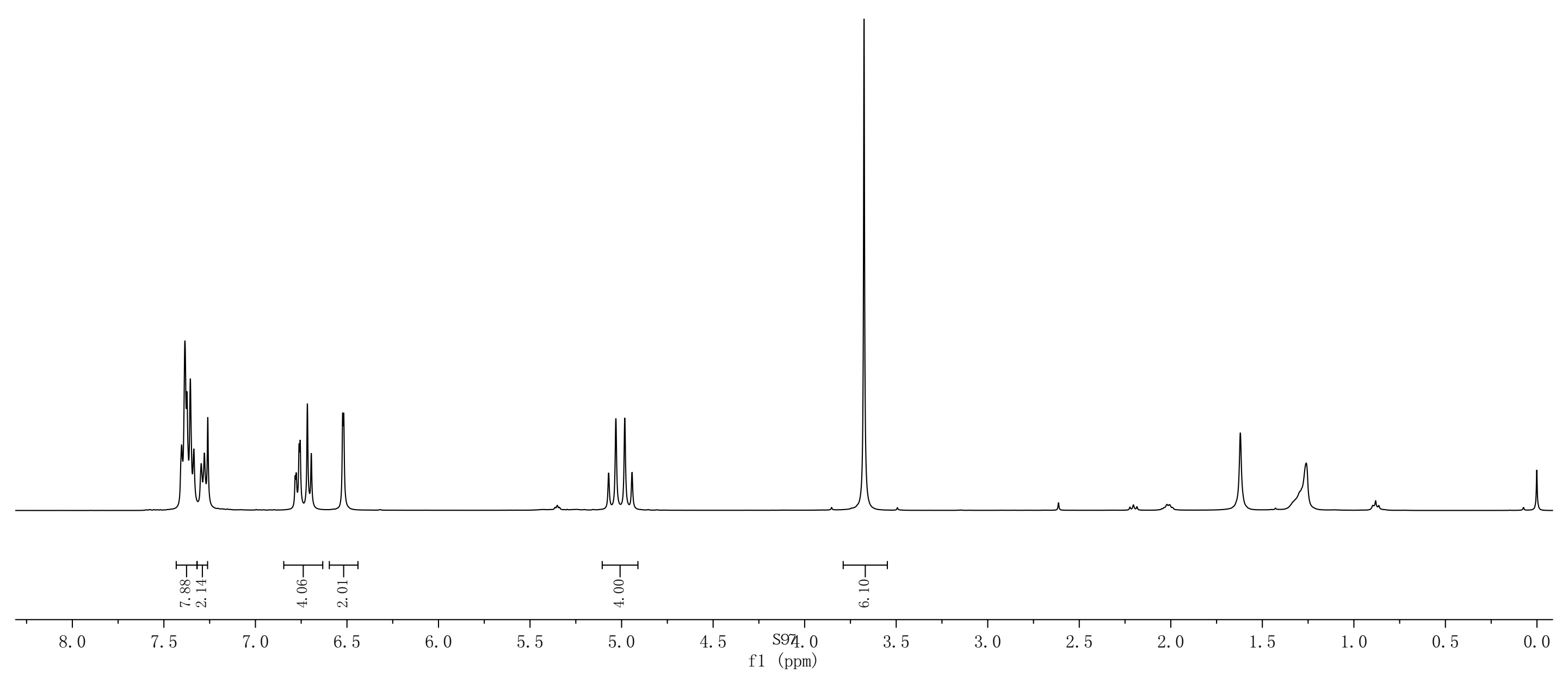




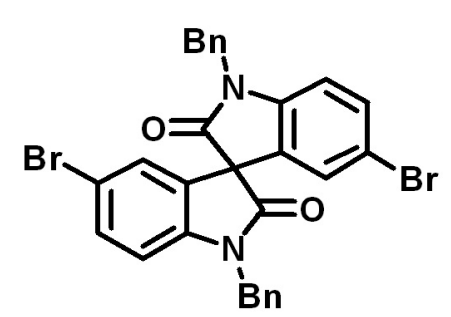

$2 \mathrm{~h}$

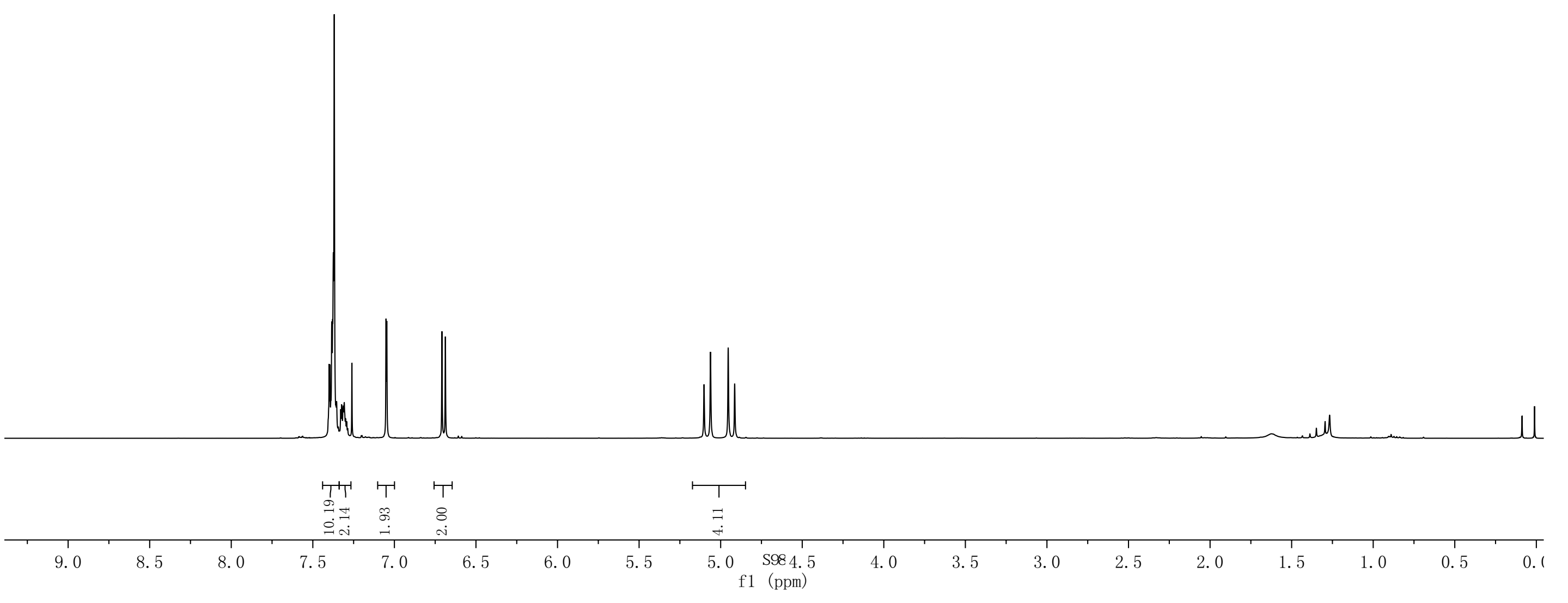


<smiles>O=C1N(Cc2ccccc2)c2ccc(Br)cc2C12C(=O)N(Cc1ccccc1)c1ccc(Br)cc12</smiles>

2h

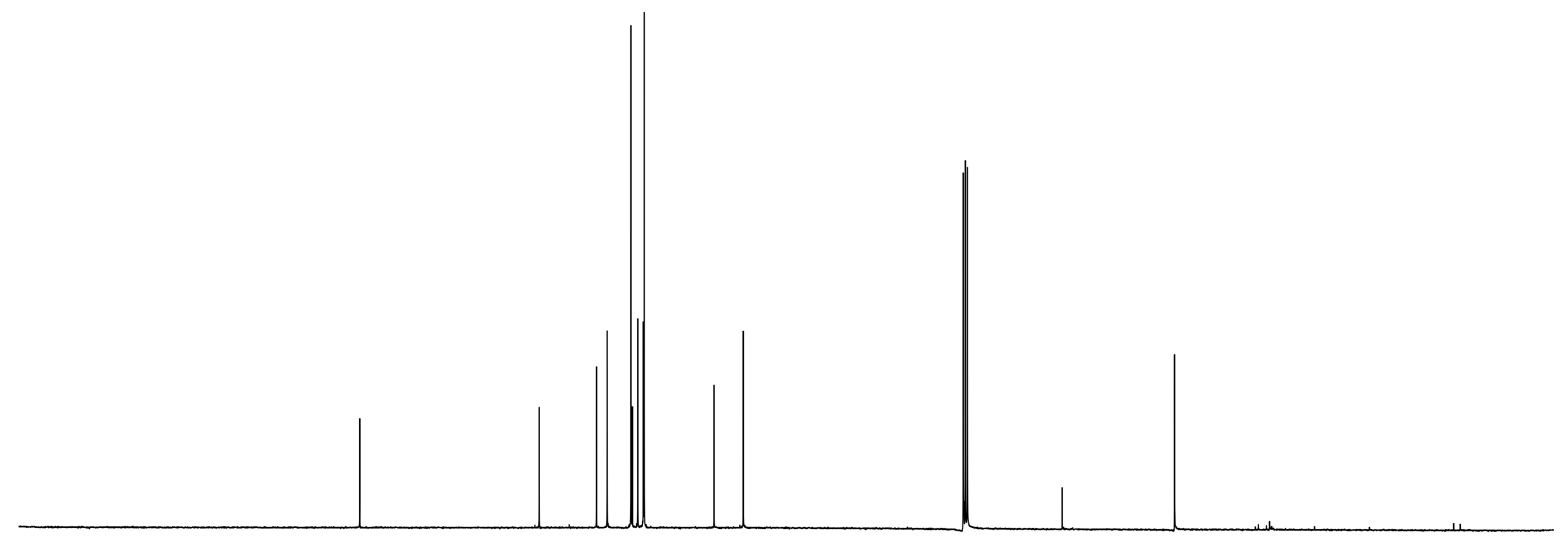

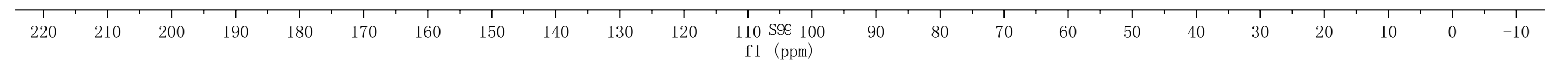




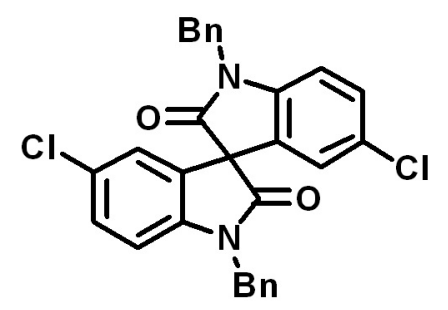

2i

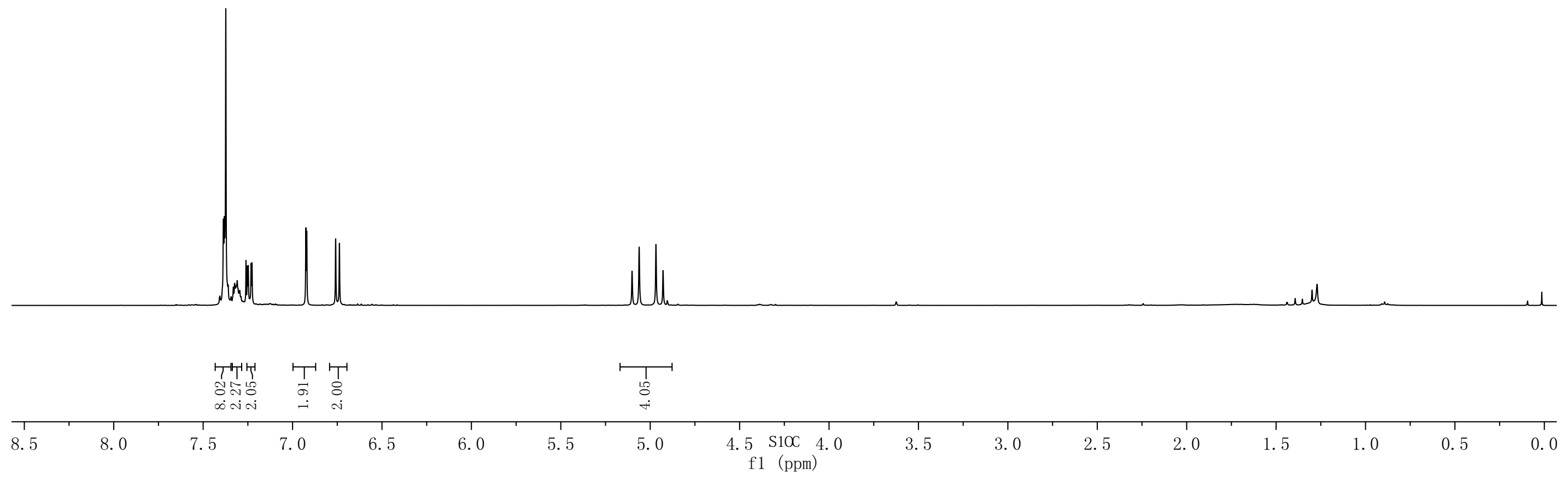



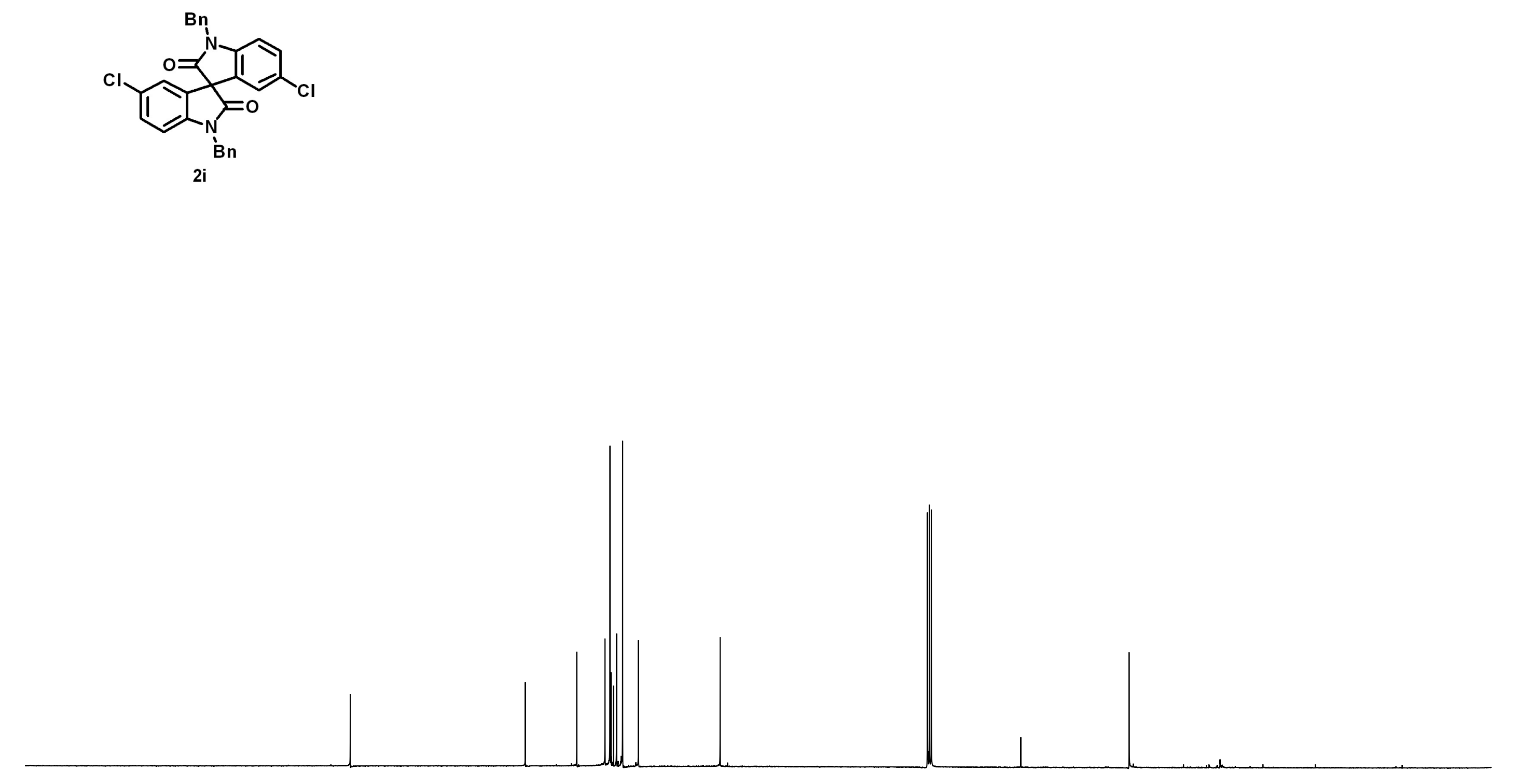

\begin{tabular}{|c|c|c|c|c|c|c|c|c|c|c|c|c|c|c|c|c|c|c|c|c|c|c|}
\hline 1 & 21 & 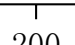 & 1 & 1 & 1 & 1 & 1 & 1 & 1 & 1 & \begin{tabular}{l|l} 
I \\
\end{tabular} & 1 & 1 & 19 & 1 & 19 & 1 & $\begin{array}{l}71 \\
20\end{array}$ & 1 & 11 & 1 & 1 \\
\hline 220 & 210 & 200 & 190 & 180 & 170 & 160 & 150 & 140 & 130 & 120 & $\begin{array}{c}110 \text { S101 } 100 \\
\text { f1 (ppm) }\end{array}$ & 90 & 80 & 70 & 60 & 50 & 40 & 30 & 20 & 10 & 0 & -10 \\
\hline
\end{tabular}


<smiles>COC(=O)c1ccc2c(c1)C1(C(=O)N(Cc3ccccc3)c3ccc(C(C)=O)cc31)C(=O)N2Cc1ccccc1</smiles>

2j

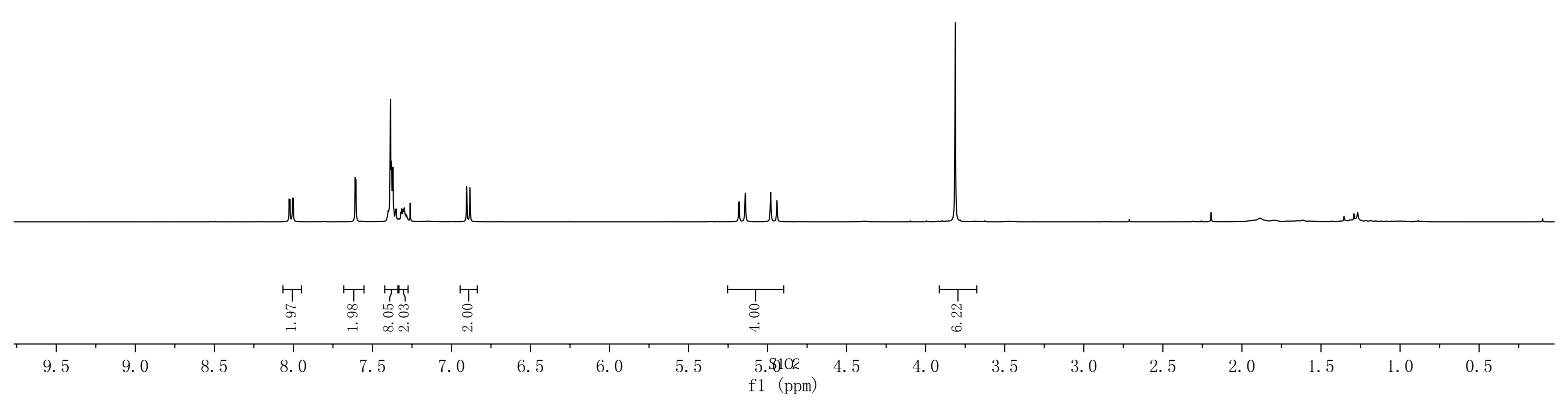


<smiles>COC(=O)c1ccc2c(c1)C1(C(=O)N2Cc2ccccc2)C(=O)N(Cc2ccccc2)c2ccc(C(=O)OC)cc21</smiles>

2j

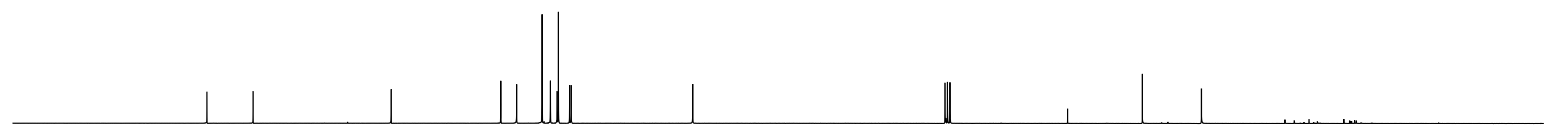

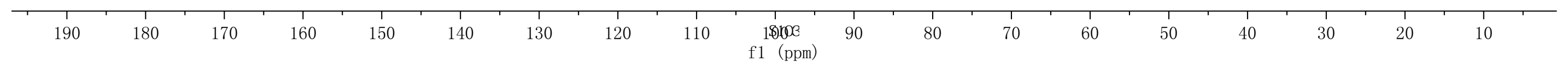




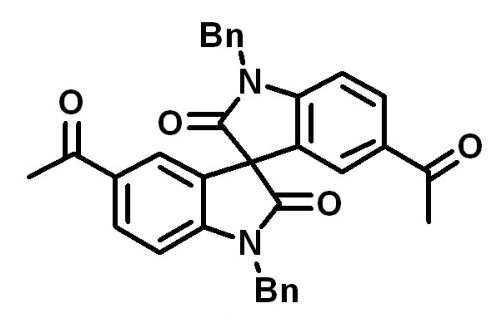

2k

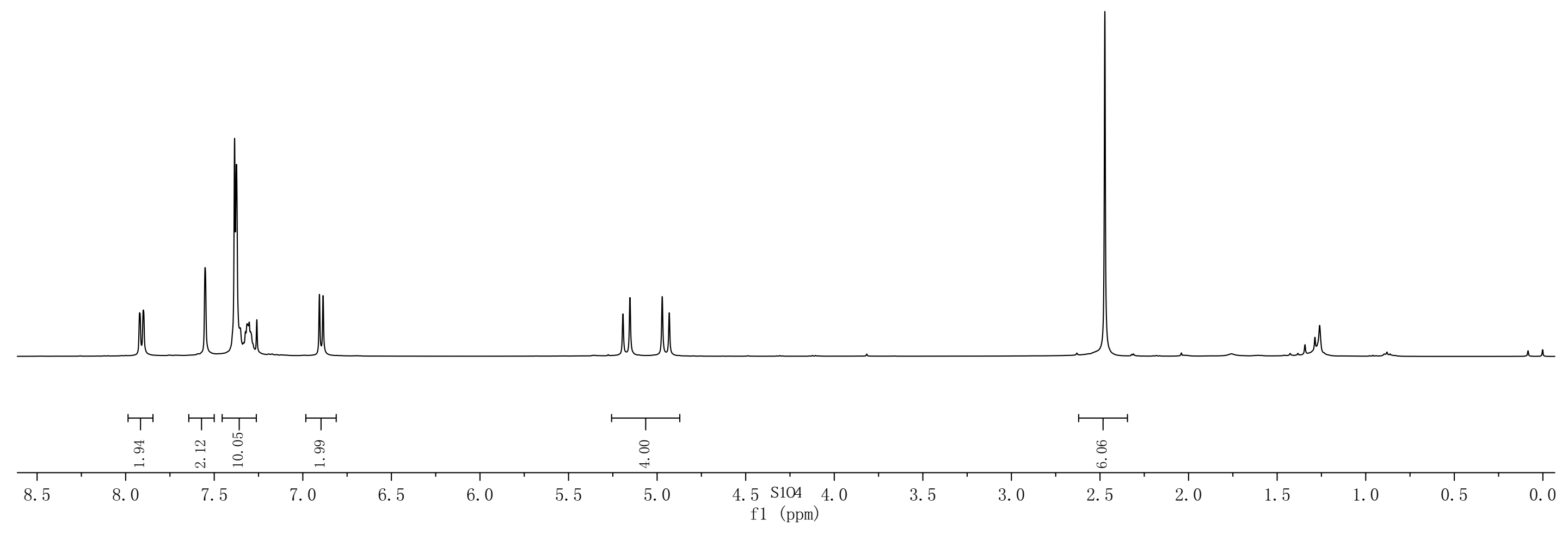



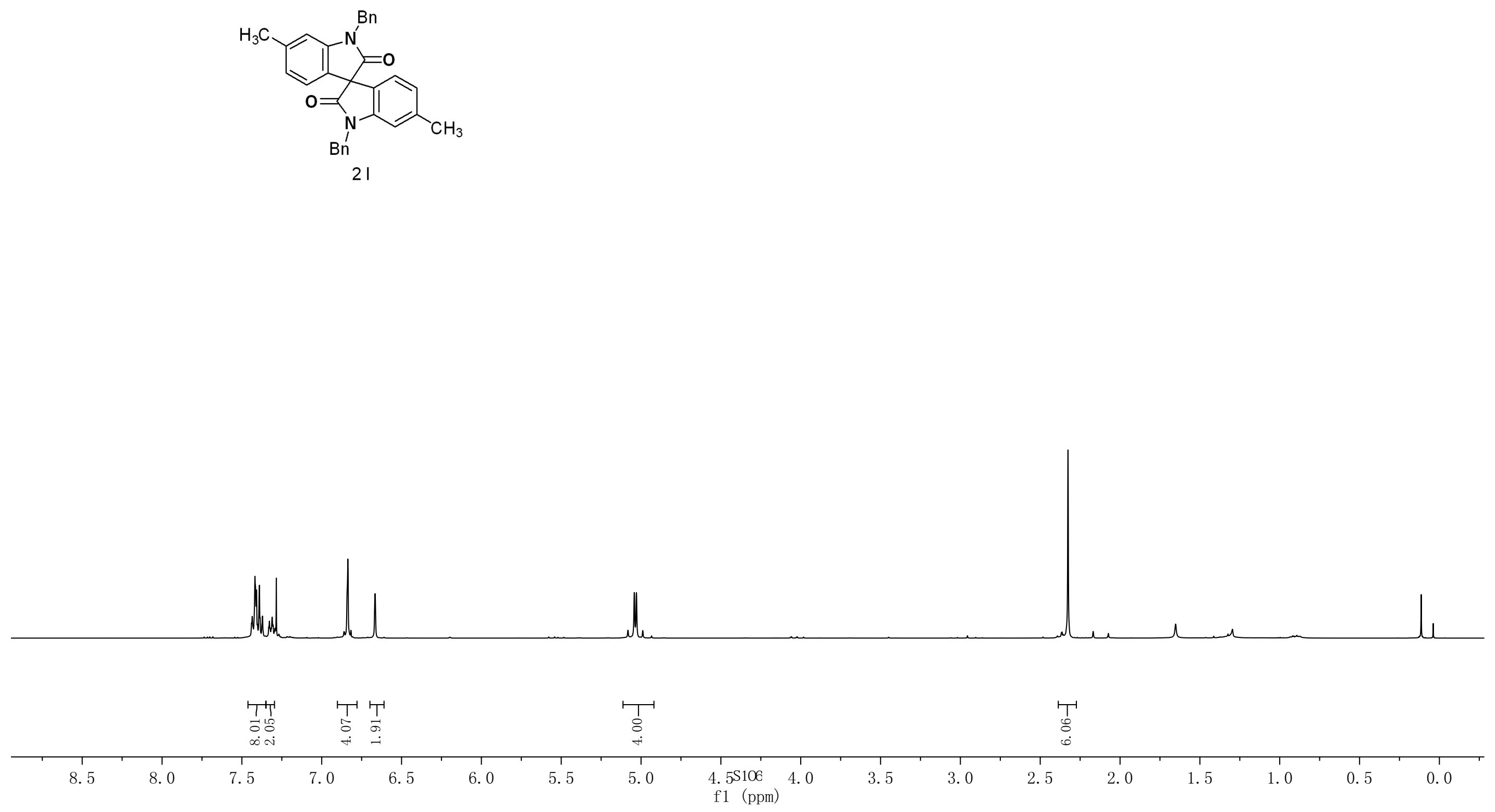

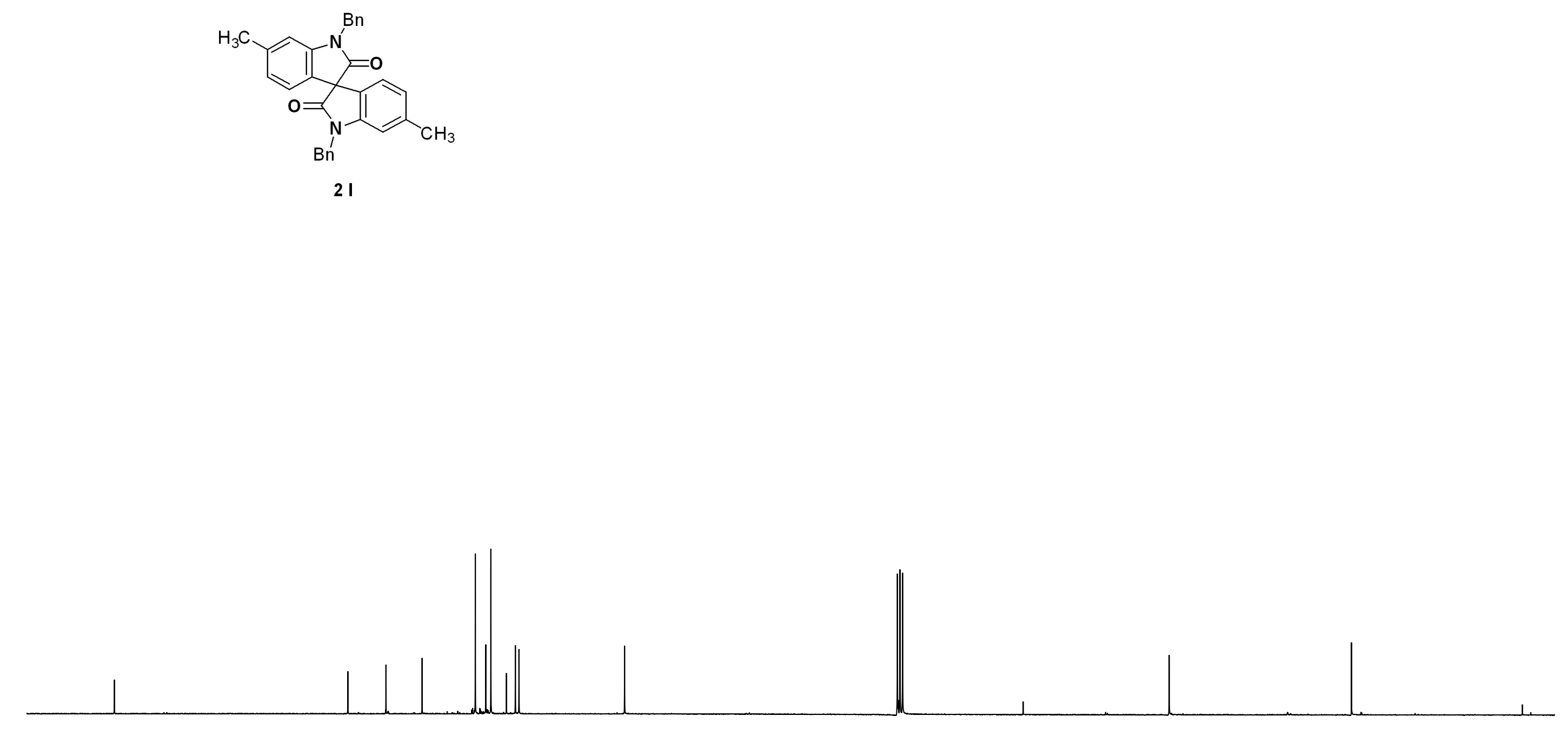

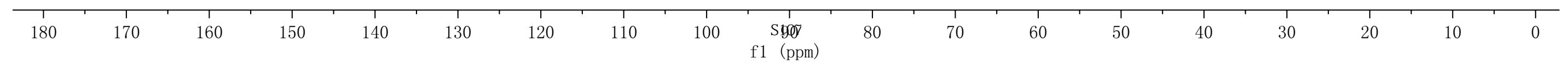



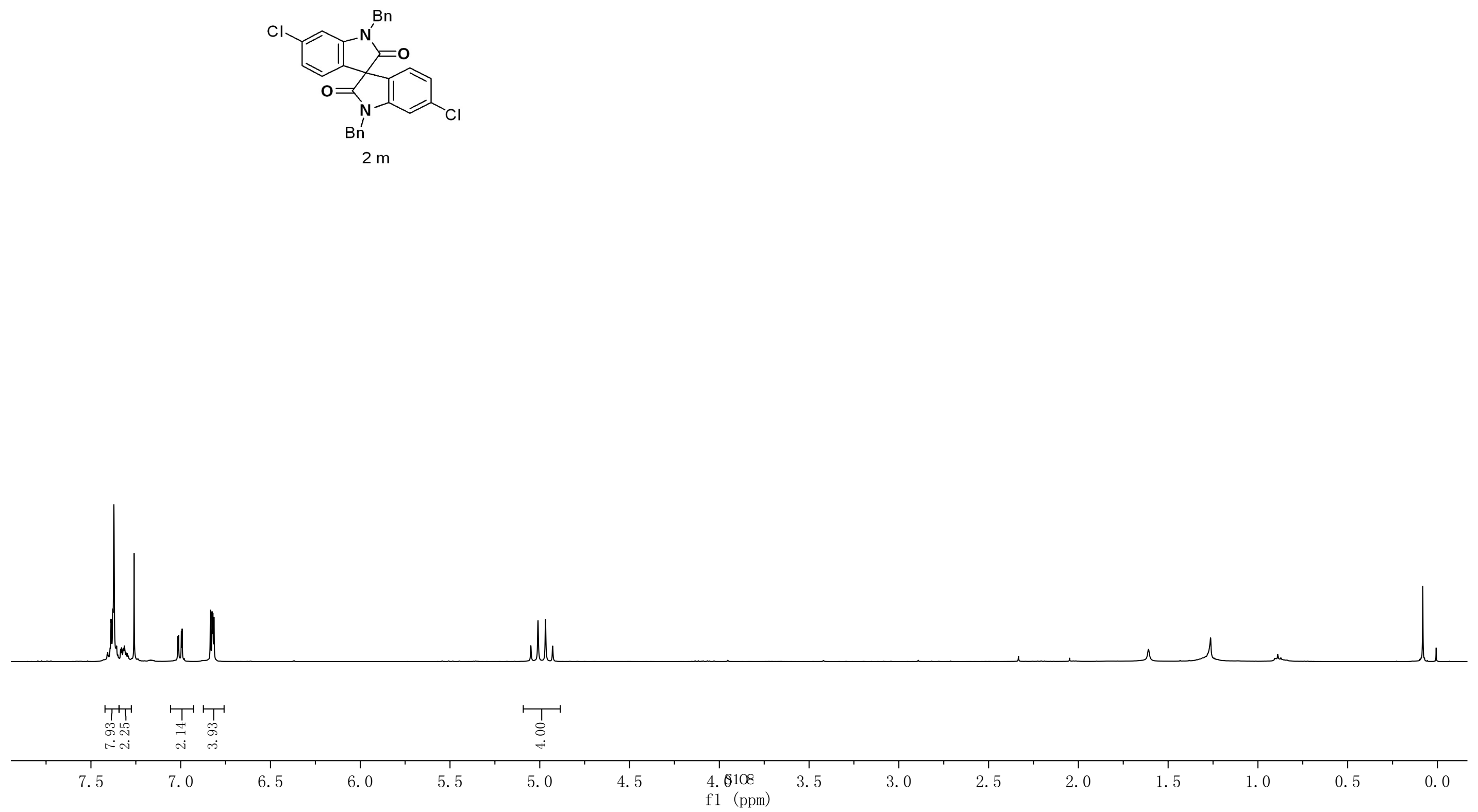
LT0339- 总

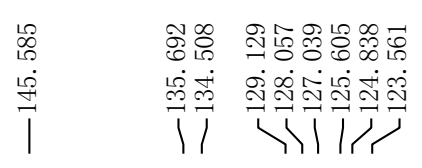

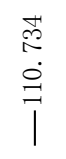

$\underset{i}{\overrightarrow{0}}$

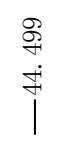
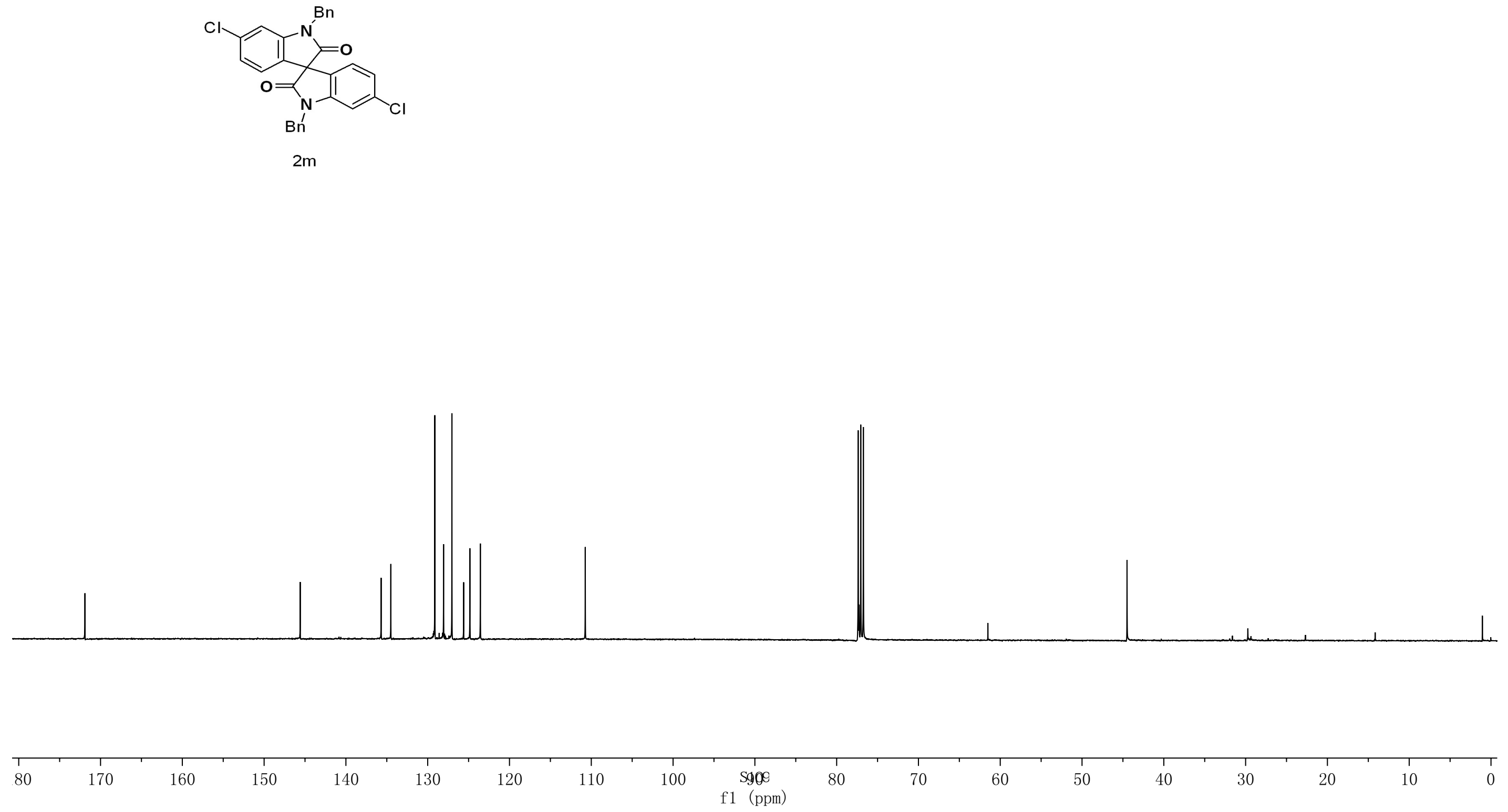


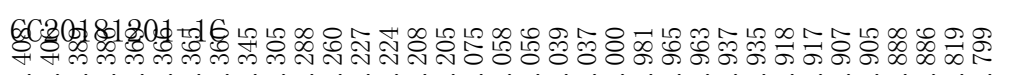

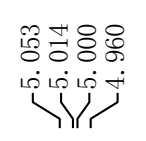

<smiles>CN1C(=O)C2(C(=O)N(Cc3ccccc3)c3ccccc32)c2ccccc21</smiles>

$4 a$
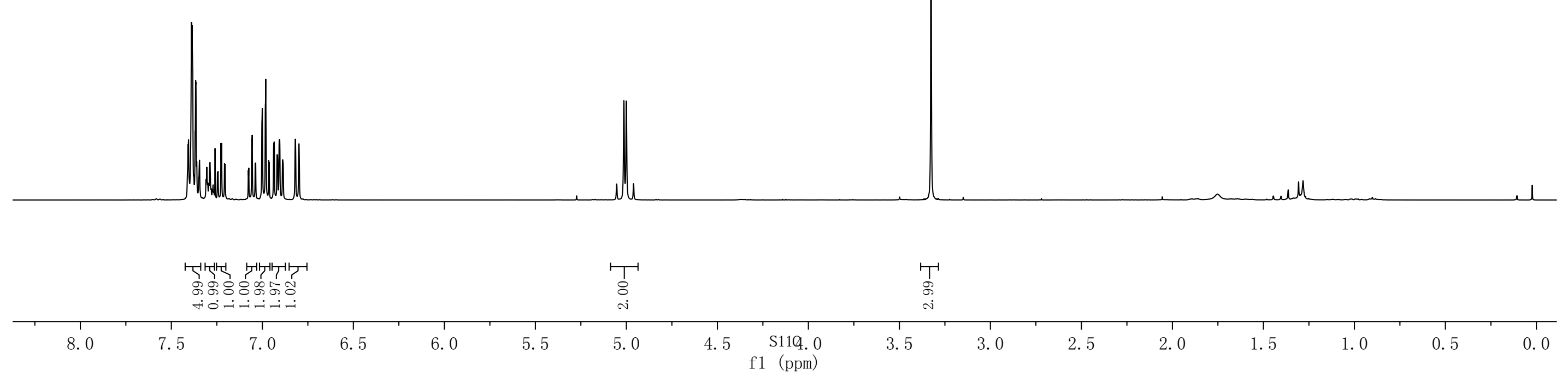

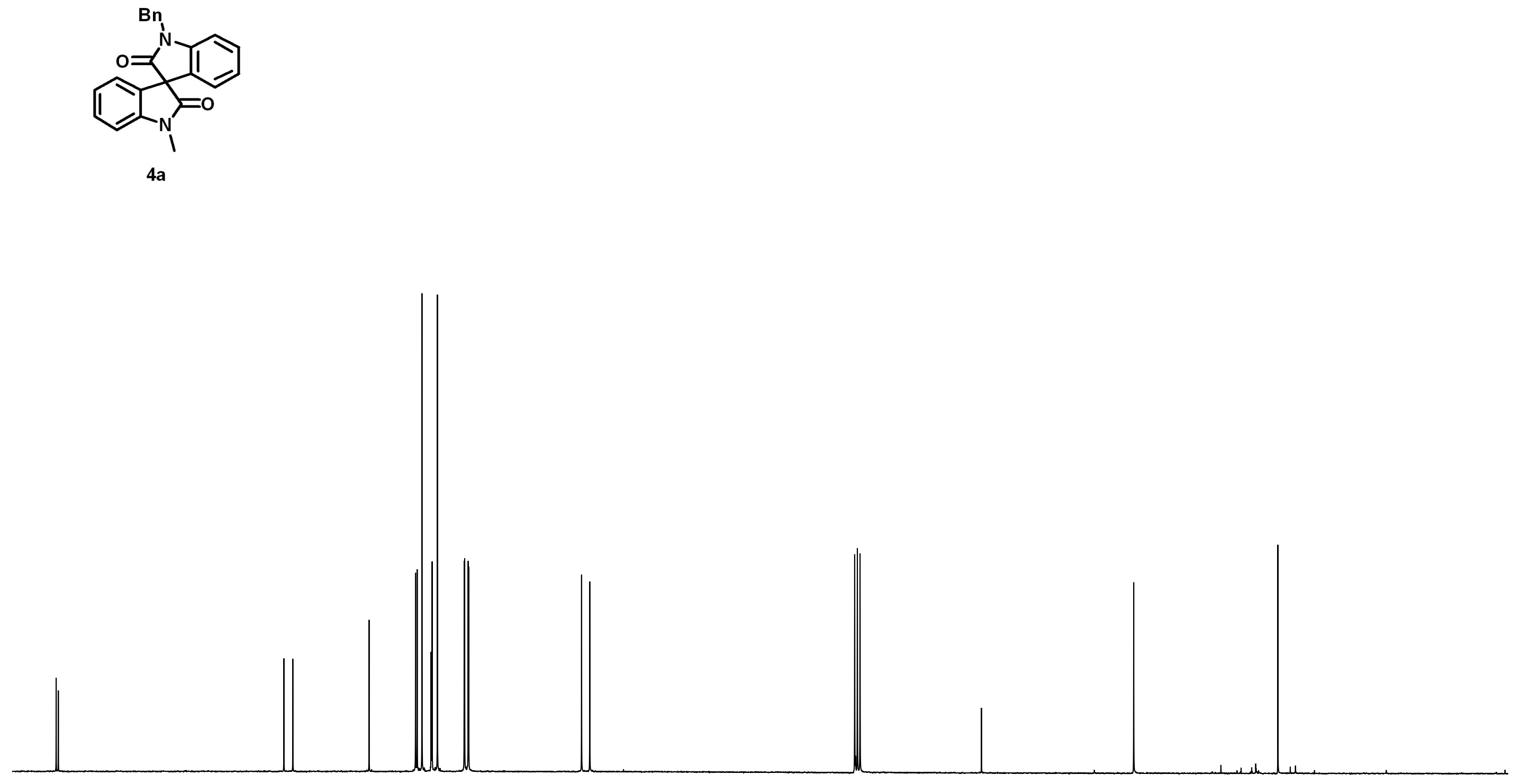

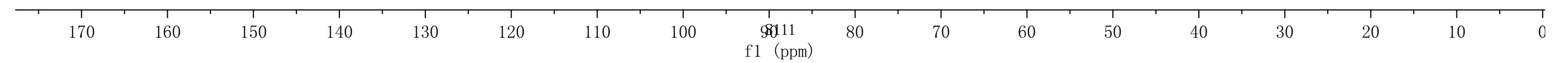



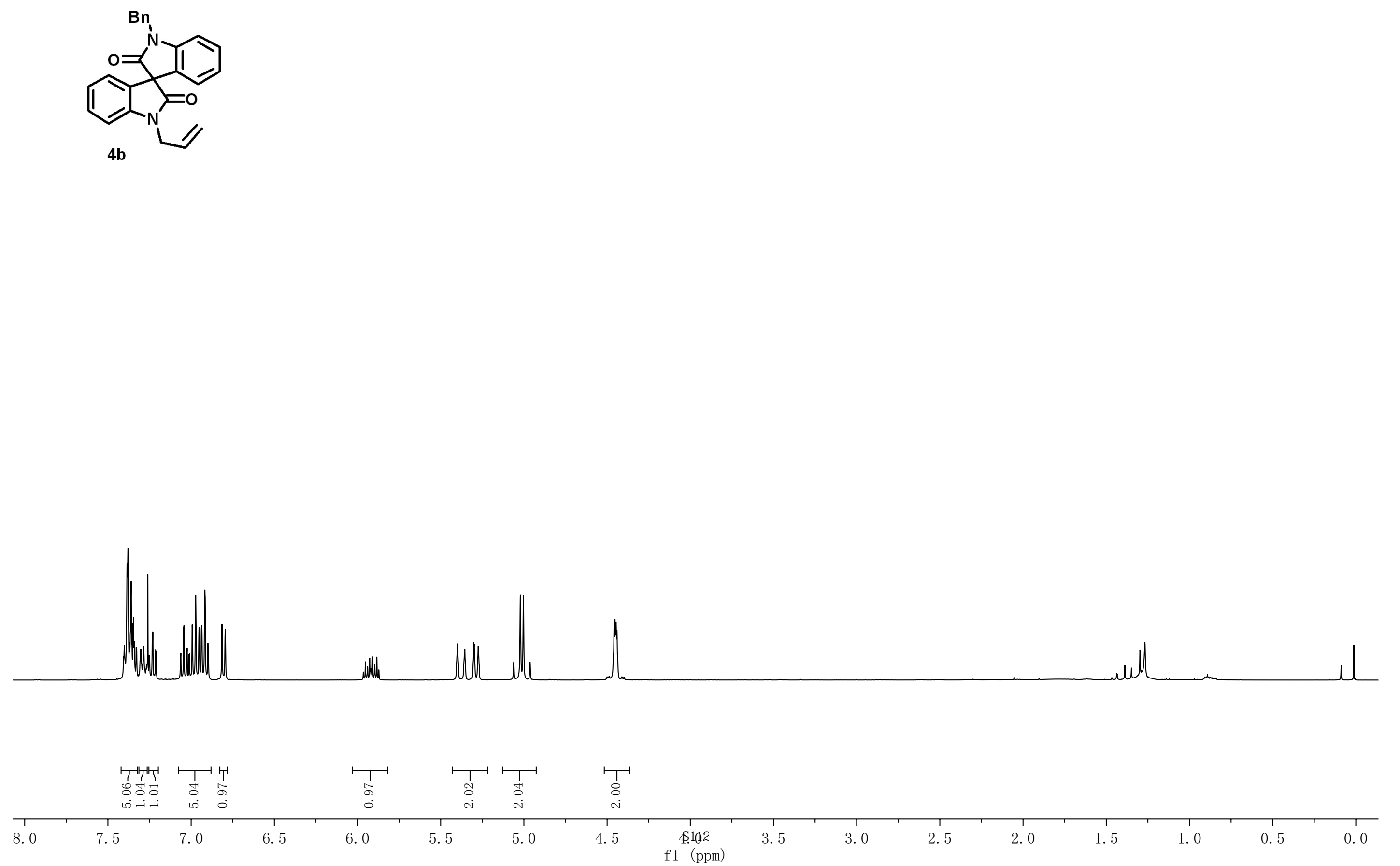

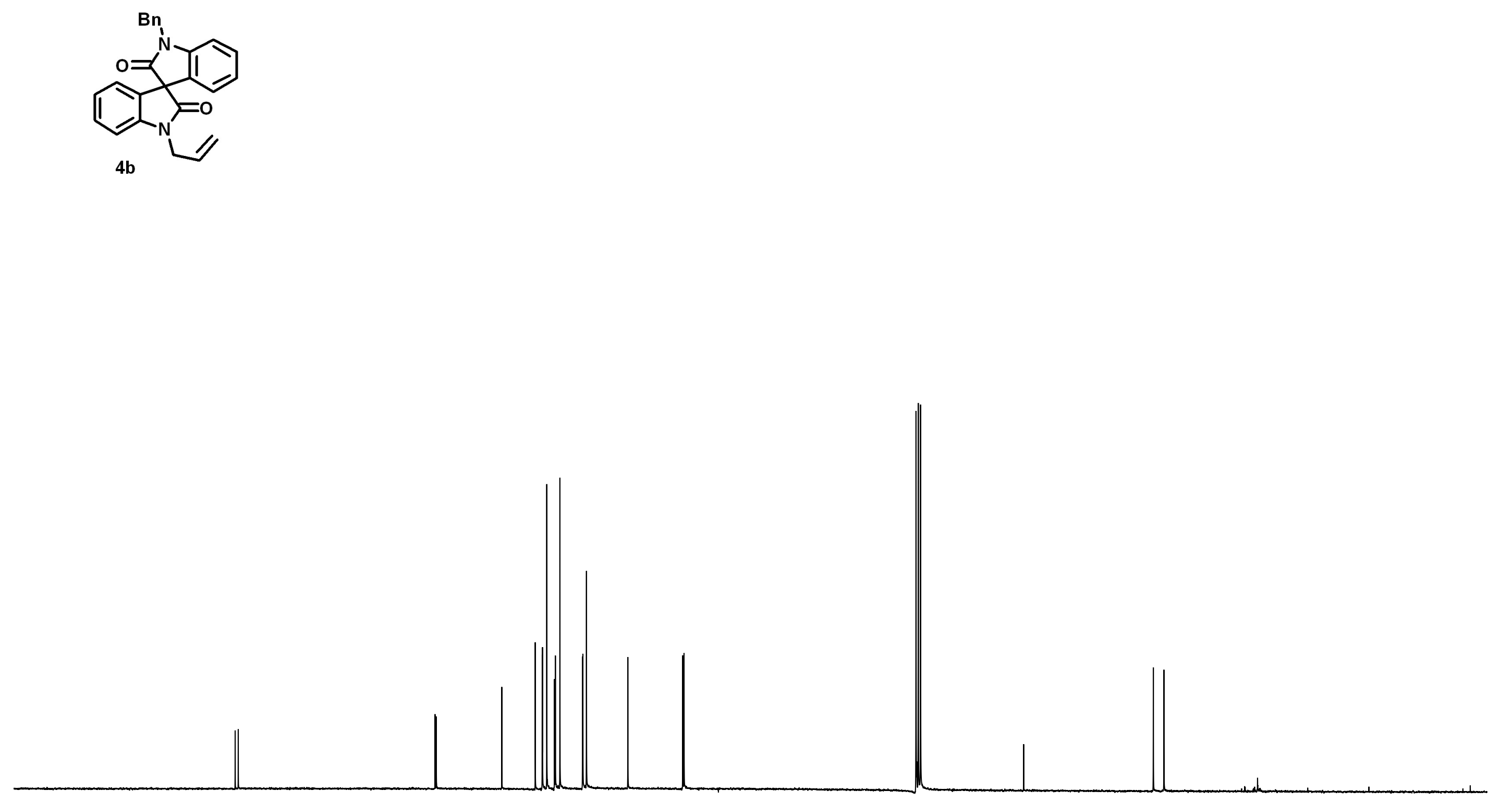


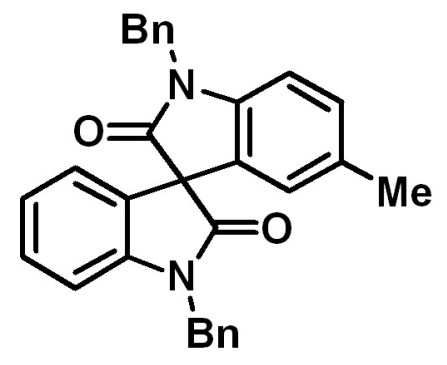

4c

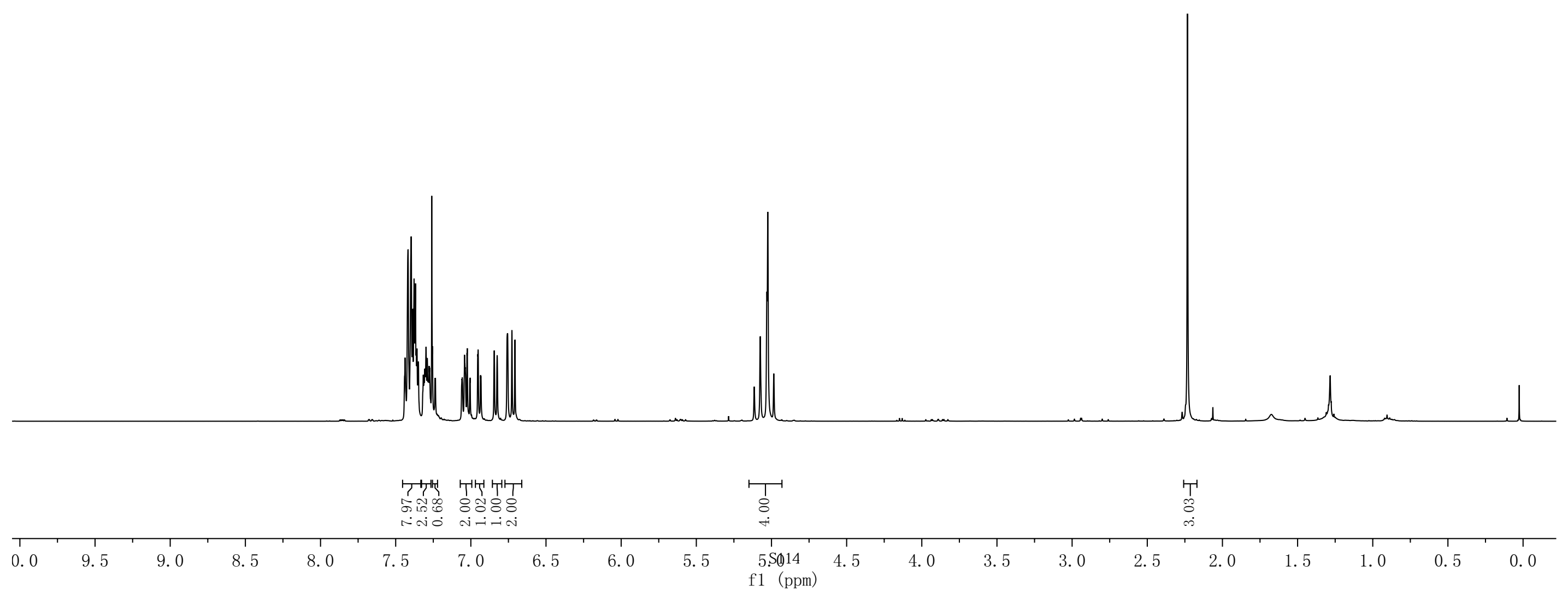




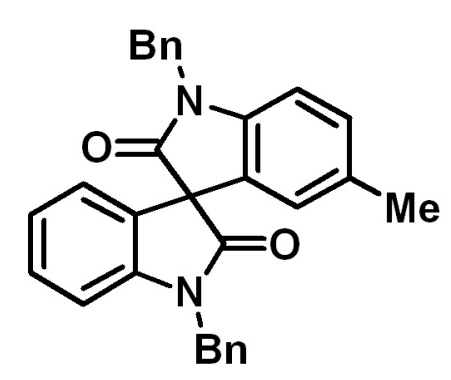

4c

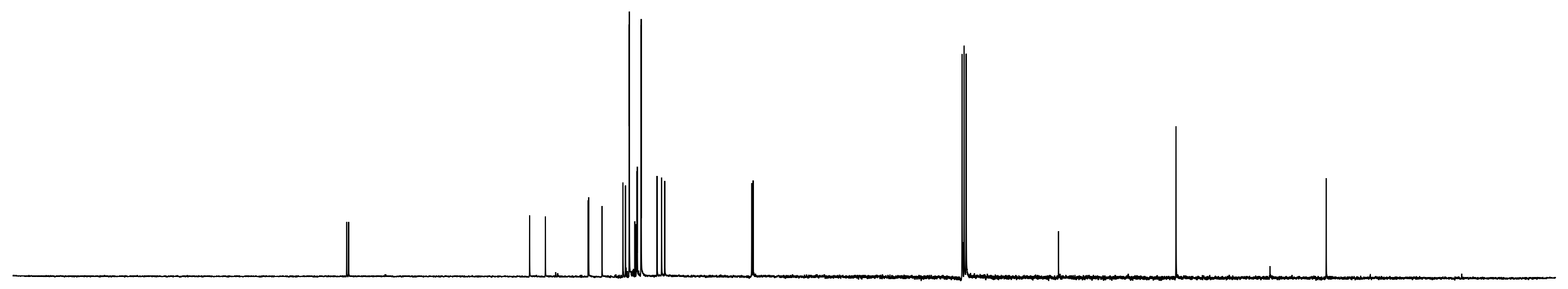


<smiles>COc1ccc2c(c1)C1(C(=O)N(Cc3ccccc3)c3ccccc31)C(=O)N2Cc1ccccc1</smiles>

4d

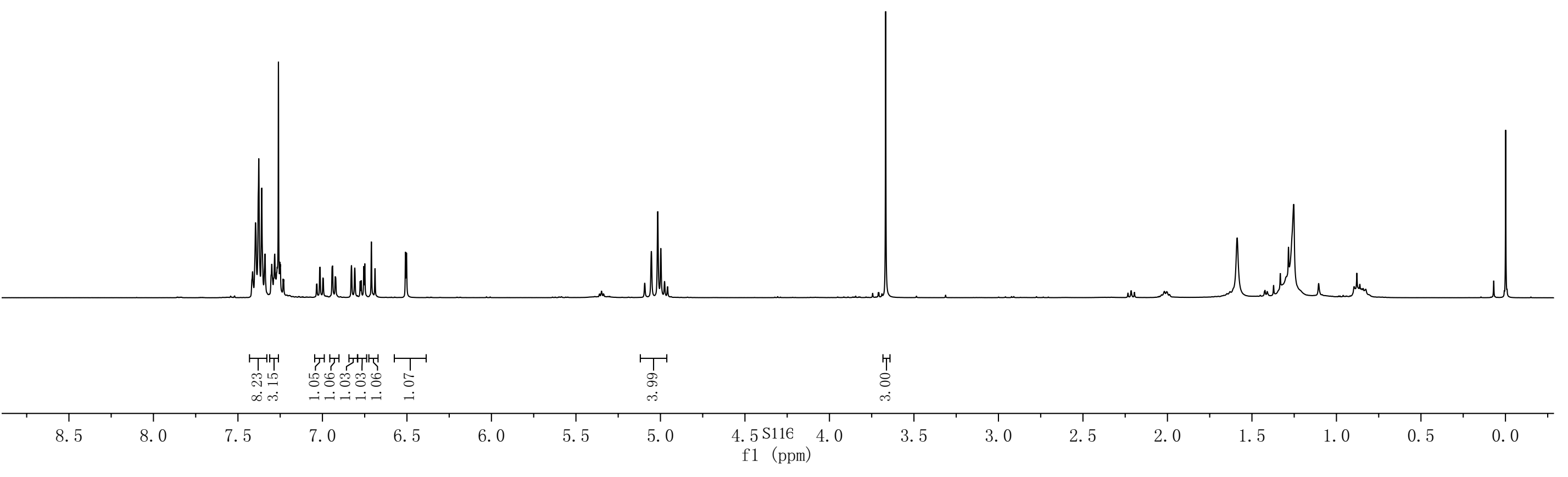


<smiles>COc1ccc2c(c1)C1(C(=O)N(Cc3ccccc3)c3ccccc31)C(=O)N2Cc1ccccc1</smiles>

4d

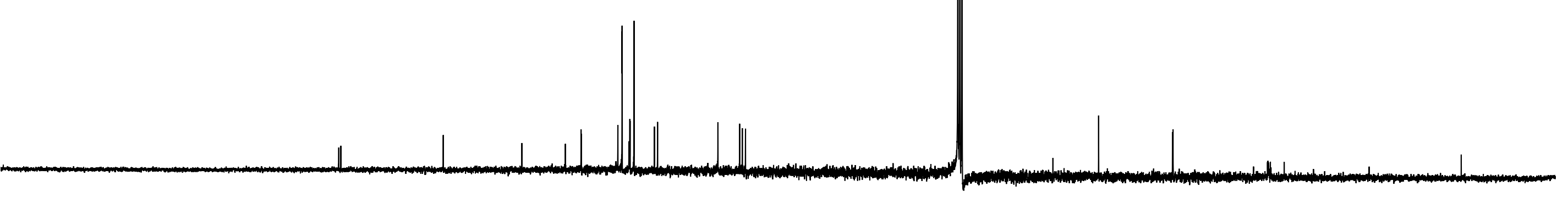

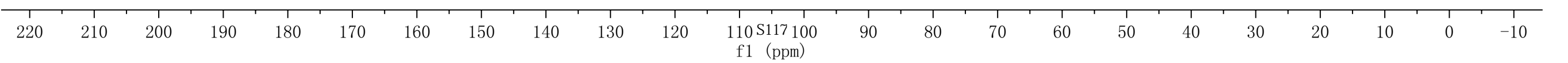



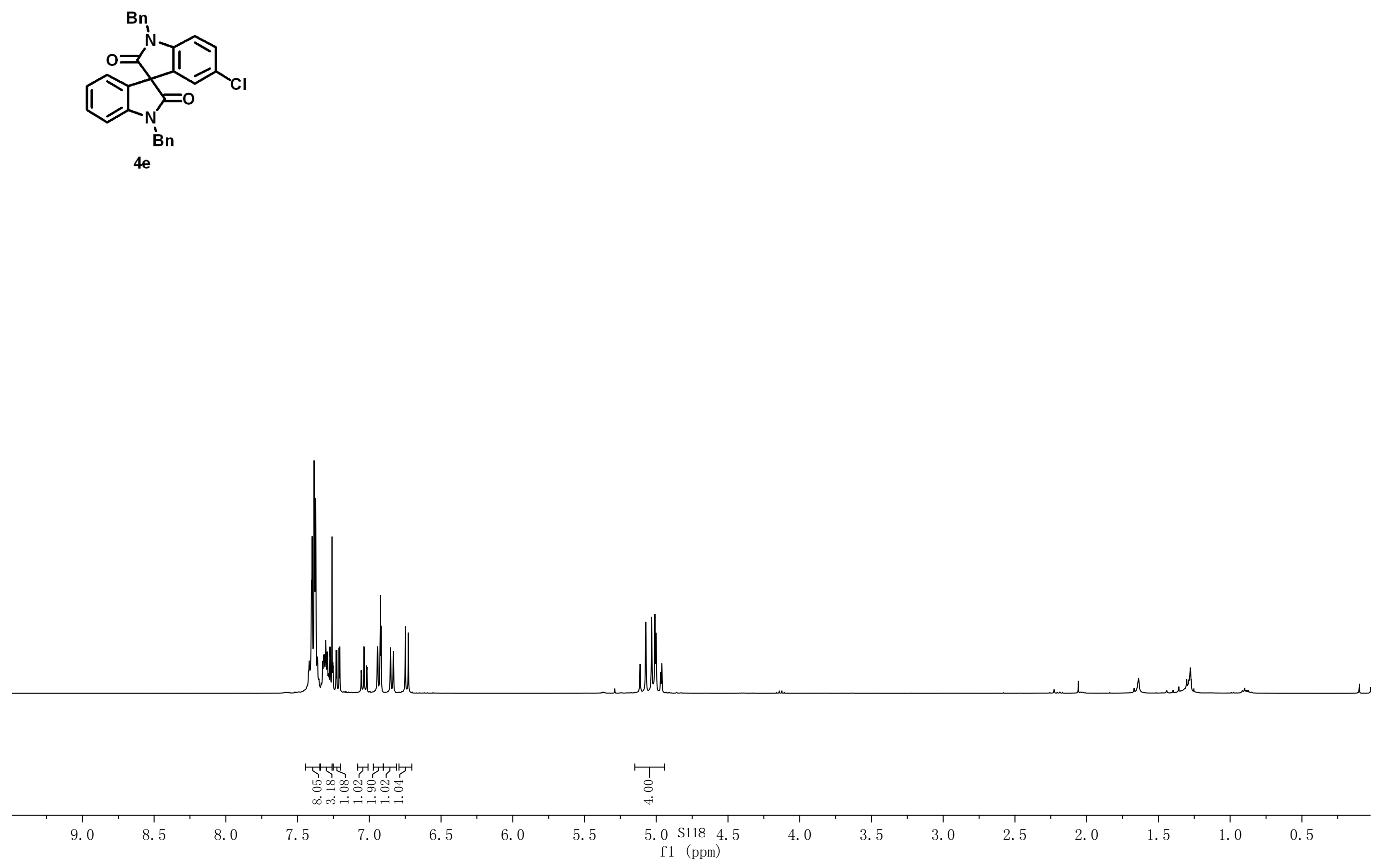

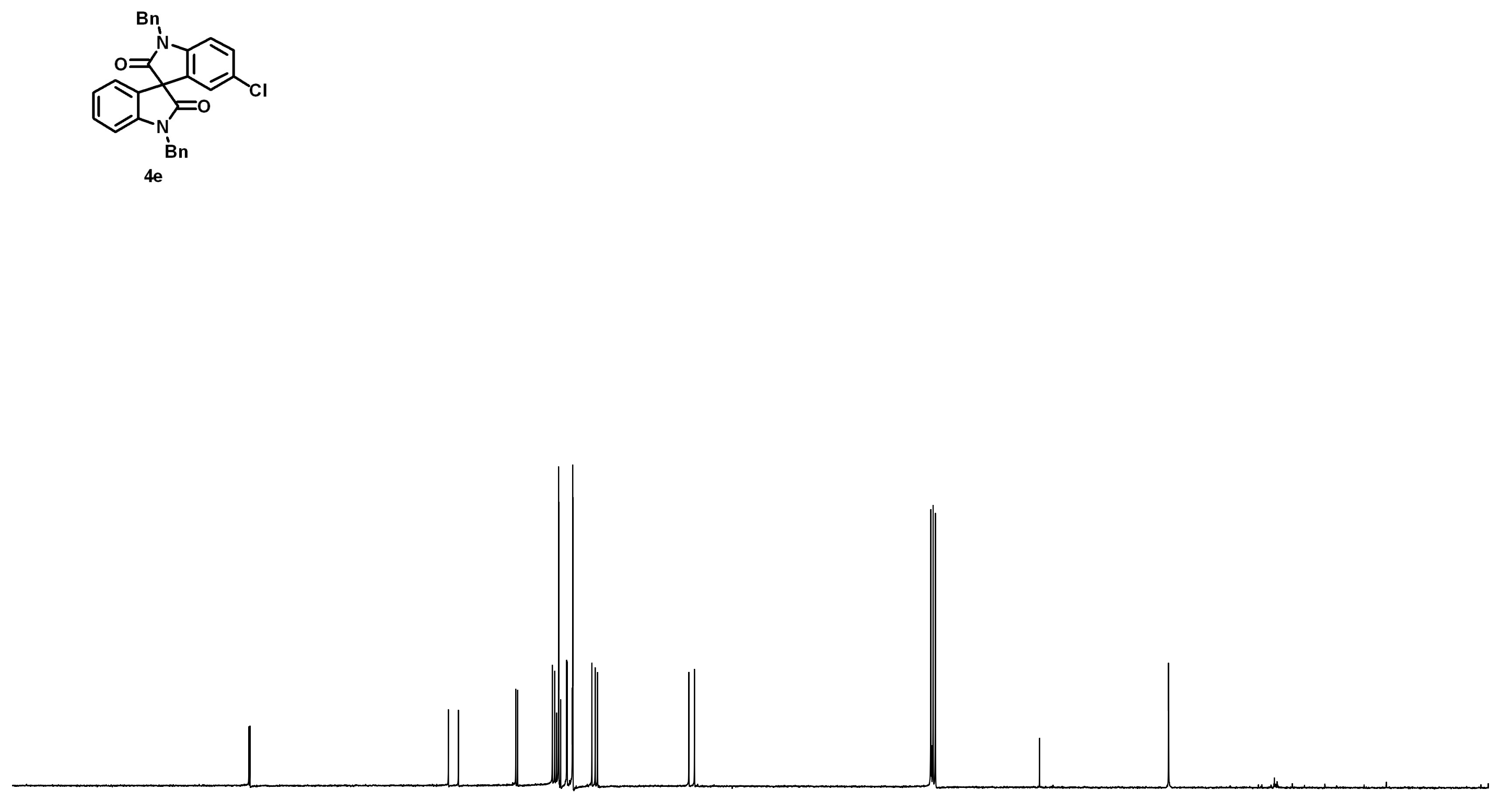

\begin{tabular}{|c|c|c|c|c|c|c|c|c|c|c|c|c|c|c|c|c|c|c|}
\hline 200 & 190 & 180 & 170 & 160 & 150 & 140 & 130 & 120 & $110 \begin{array}{r}\mathrm{S} 11 \mathrm{p} 00 \\
\mathrm{f} 1 \quad(\mathrm{ppm})\end{array}$ & 90 & 80 & 70 & 60 & 50 & 40 & 30 & 20 & 10 \\
\hline
\end{tabular}



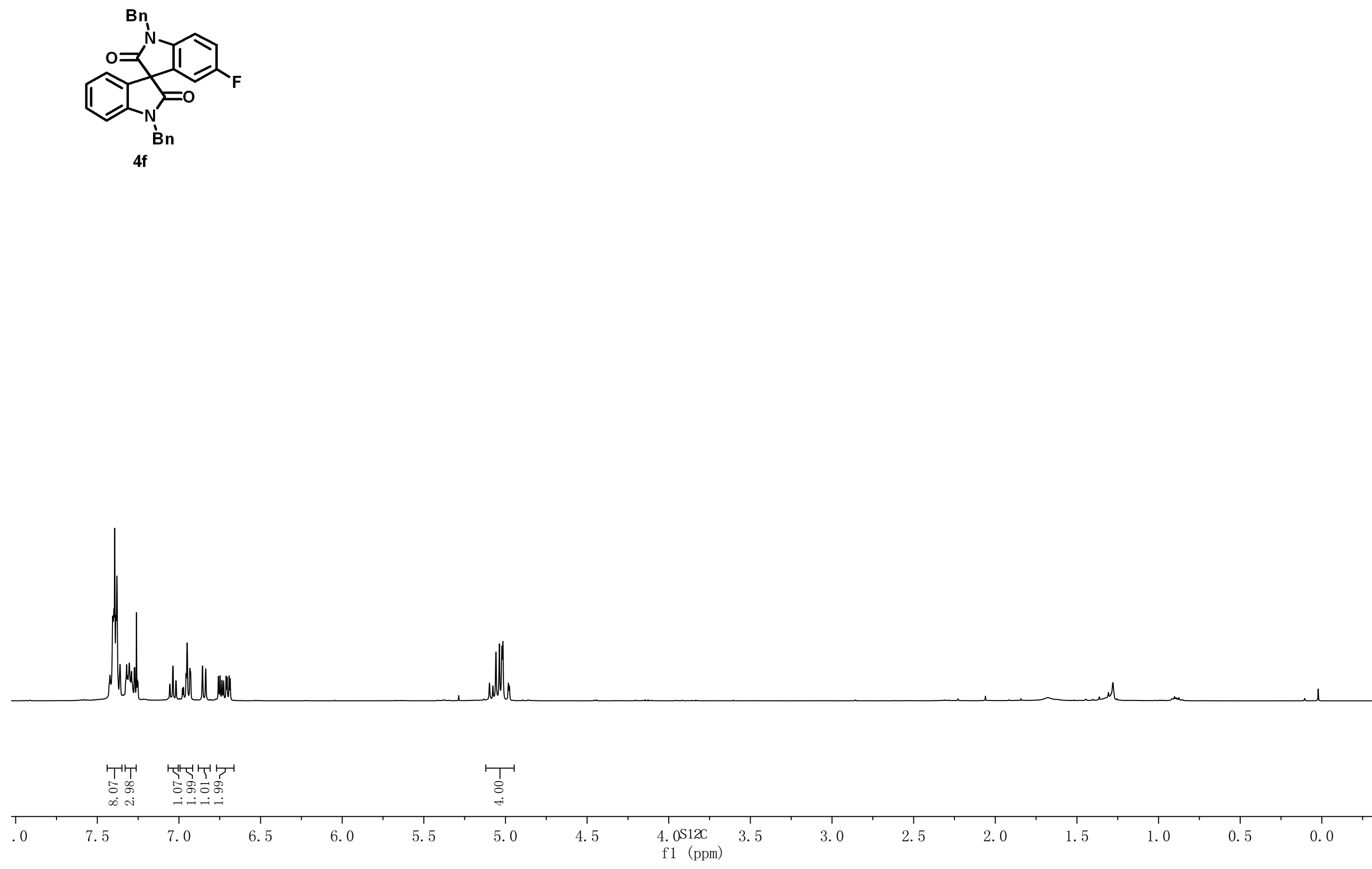


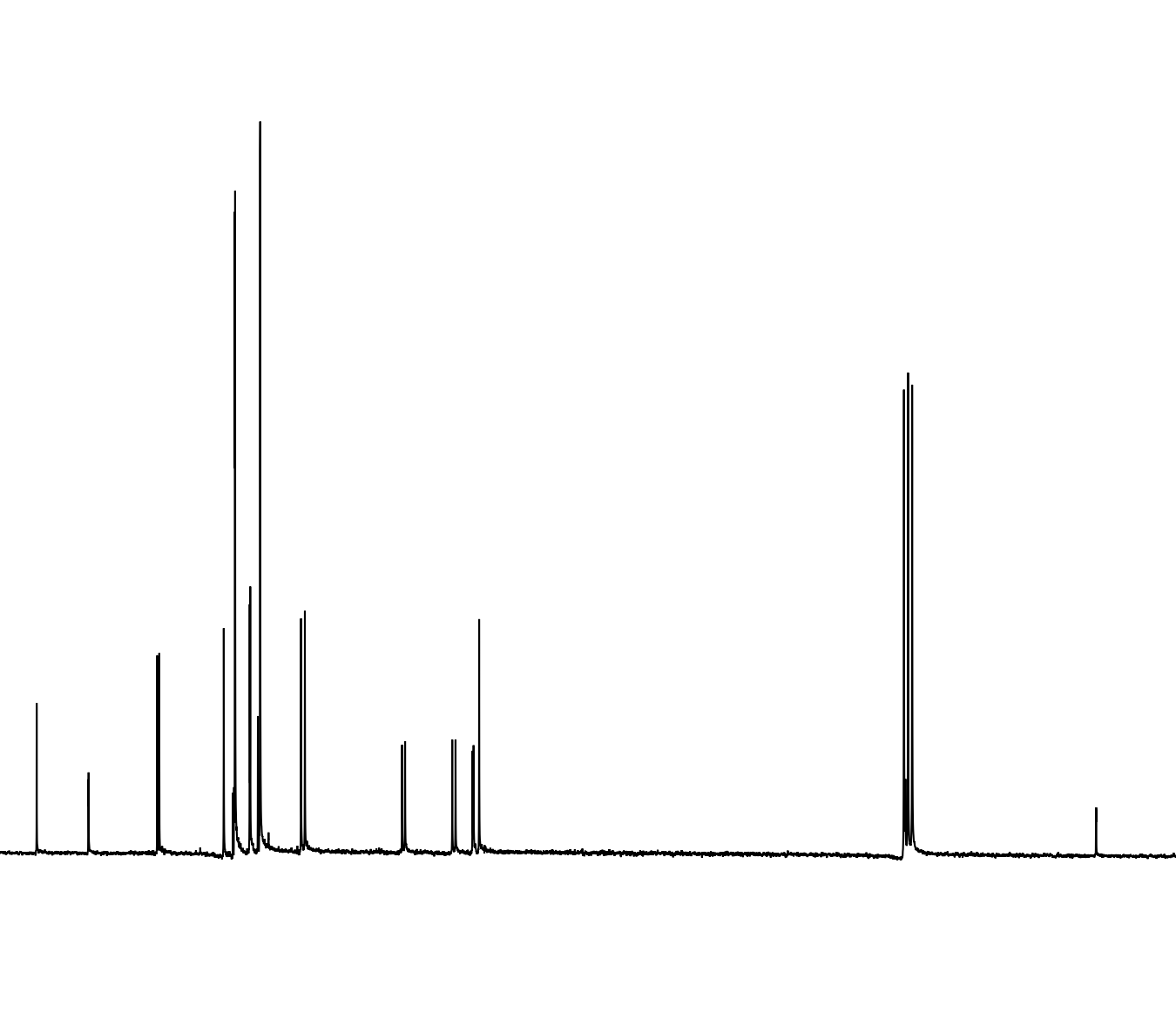




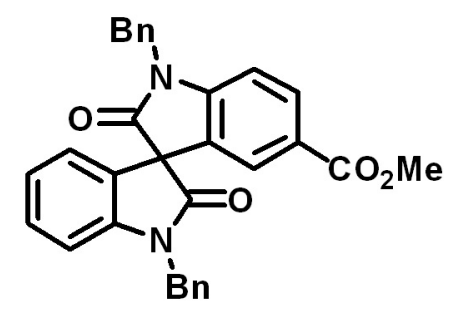

$4 \mathrm{~g}$

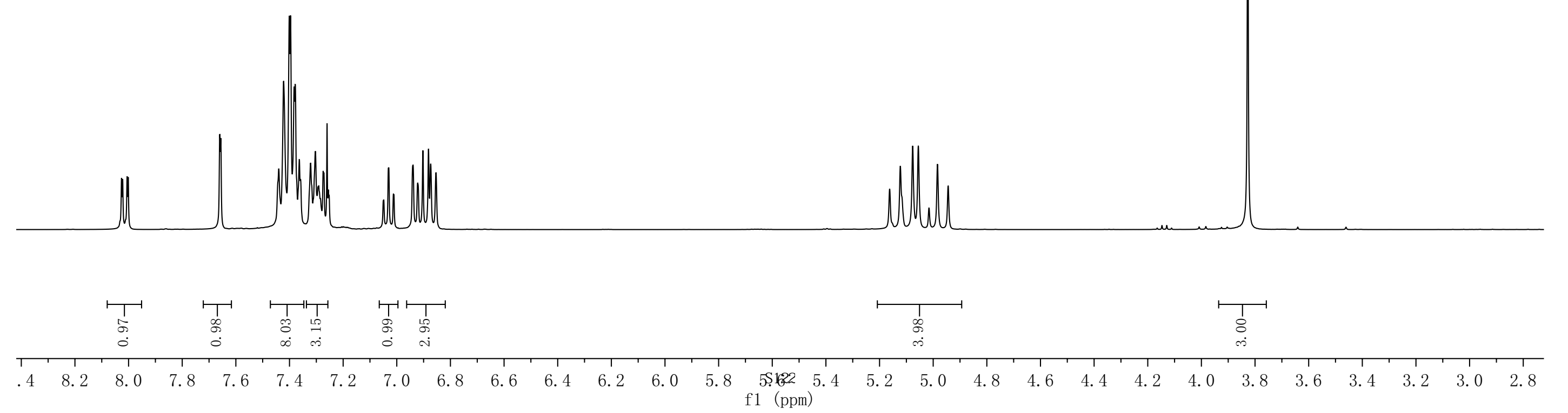


<smiles>CC(=O)c1ccc2c(c1)C1(C(=O)N(Cc3ccccc3)c3ccccc31)C(=O)N2Cc1ccccc1</smiles>

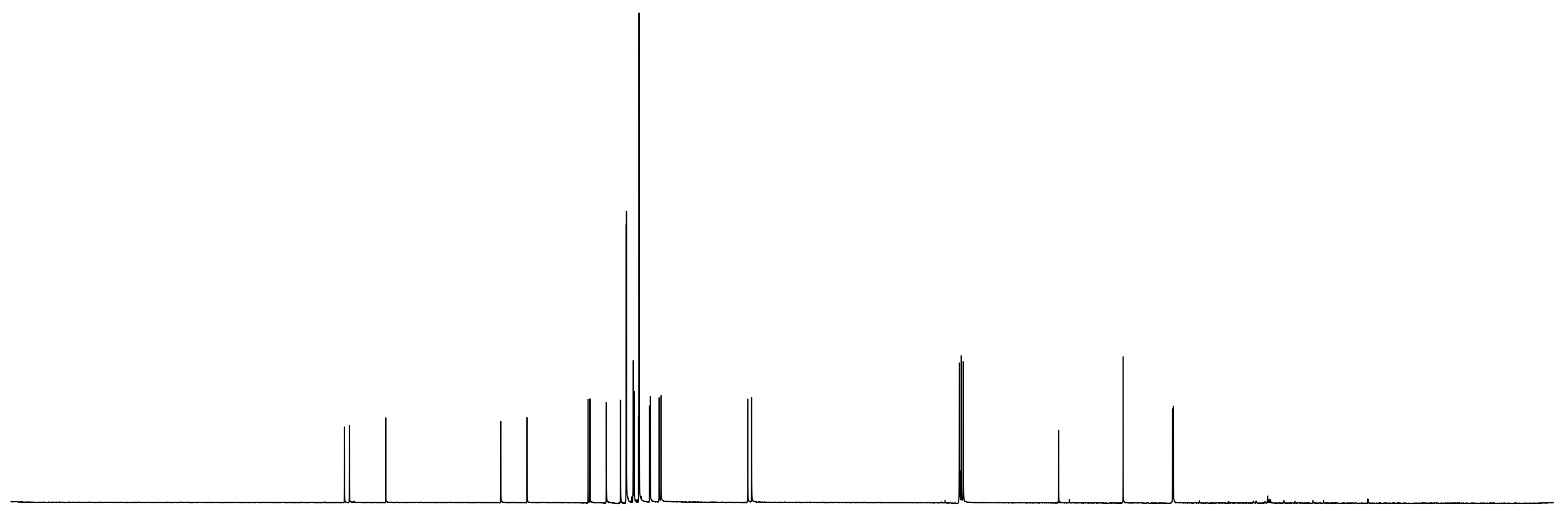




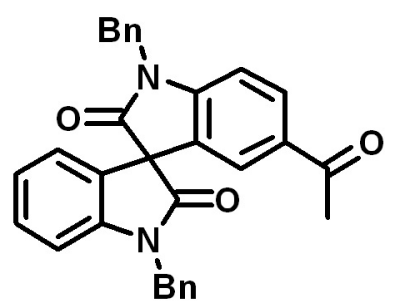

4h
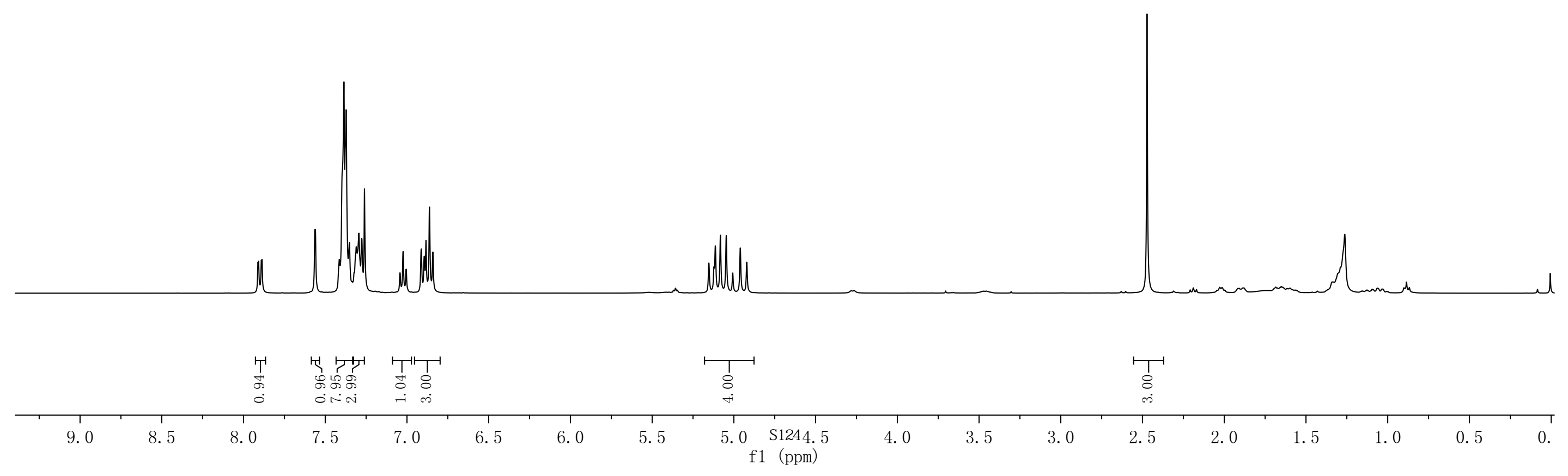
<smiles>CC(=O)c1ccc2c(c1)C1(C(=O)N(Cc3ccccc3)c3ccccc31)C(=O)N2Cc1ccccc1</smiles>

4h

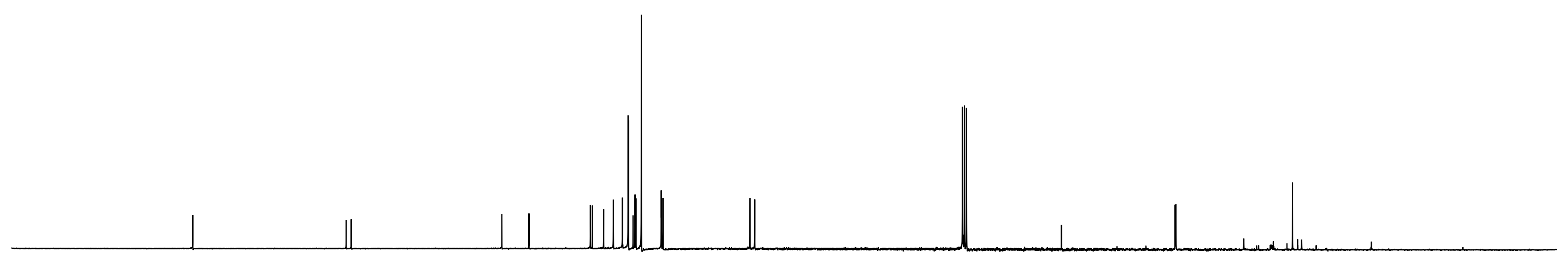




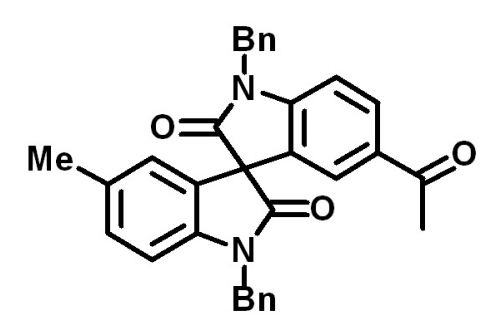

$4 i$

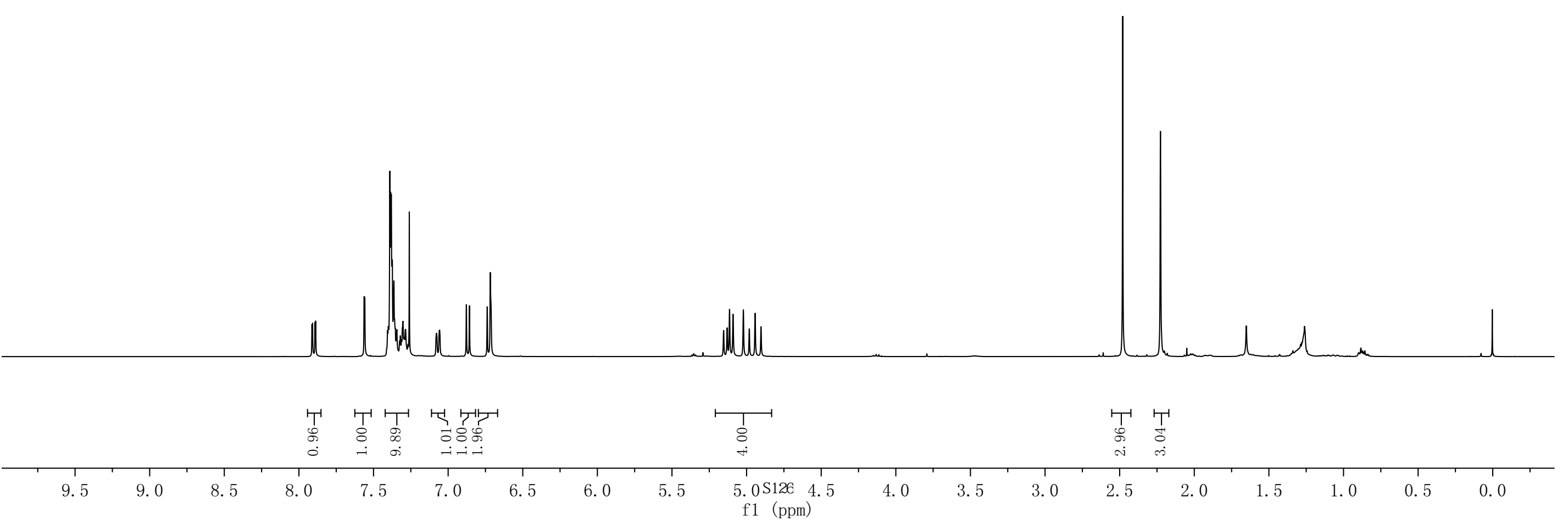


LT201802075

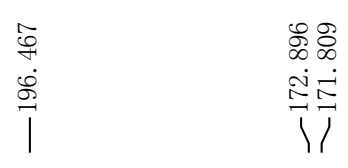

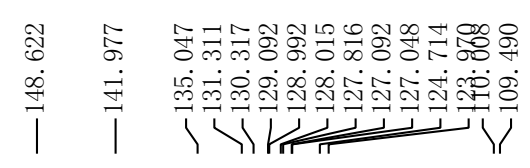

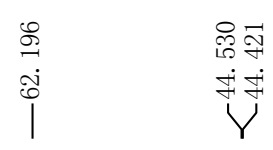

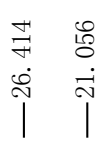

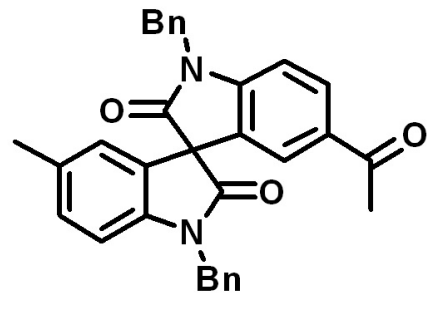

$4 i$
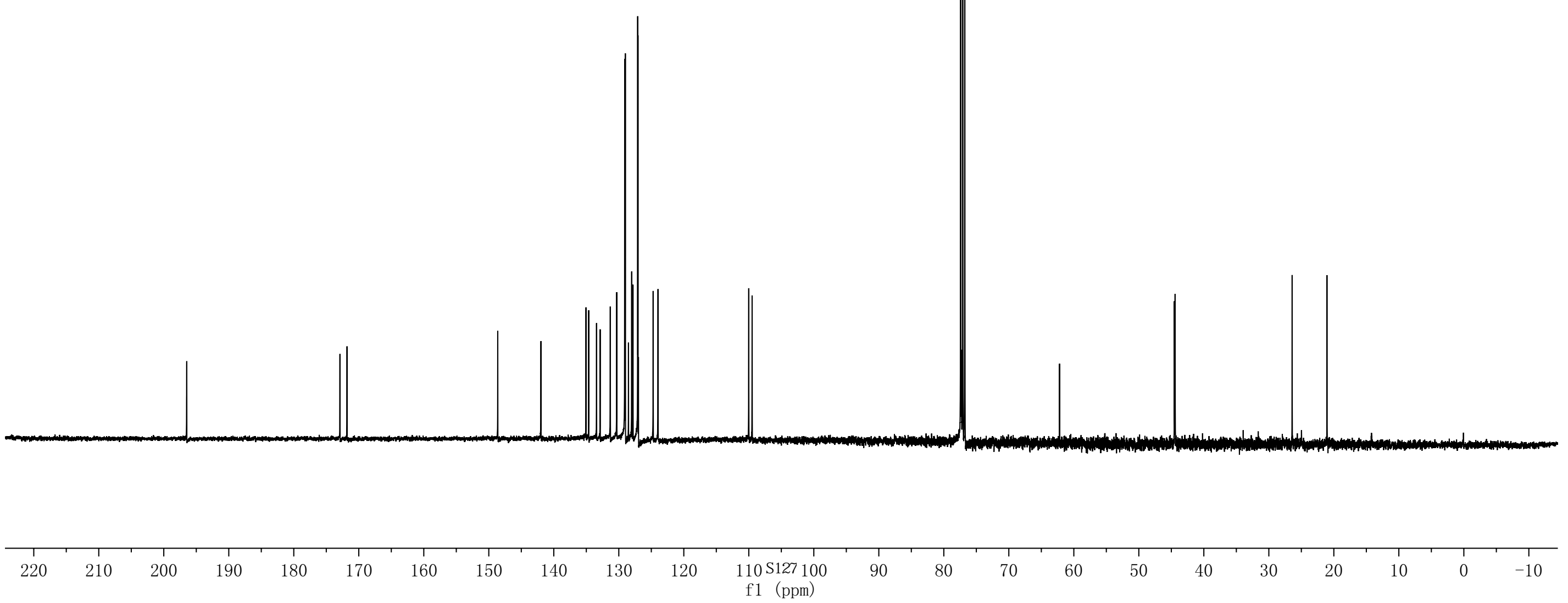
 \\ LabSolutions 分析报告}

《样品信息>

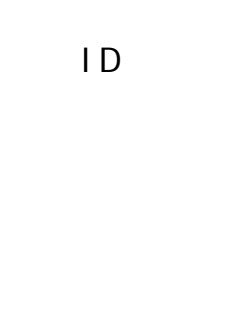

$:$ It

: LTJIAJI - OD H 30. I cd

: OD H 30\% 60mi n. I cm

1- 1

$1 \mathrm{uL}$

2019/1/21 19: 04: 30

样品类型：未知

2019/1/21 20: 32: 57

分析者 : Syst em Admi ni st r at or

处理者 : Syst em Admi ni st $r$ at or

<色谱图>

$\mathrm{nV}$

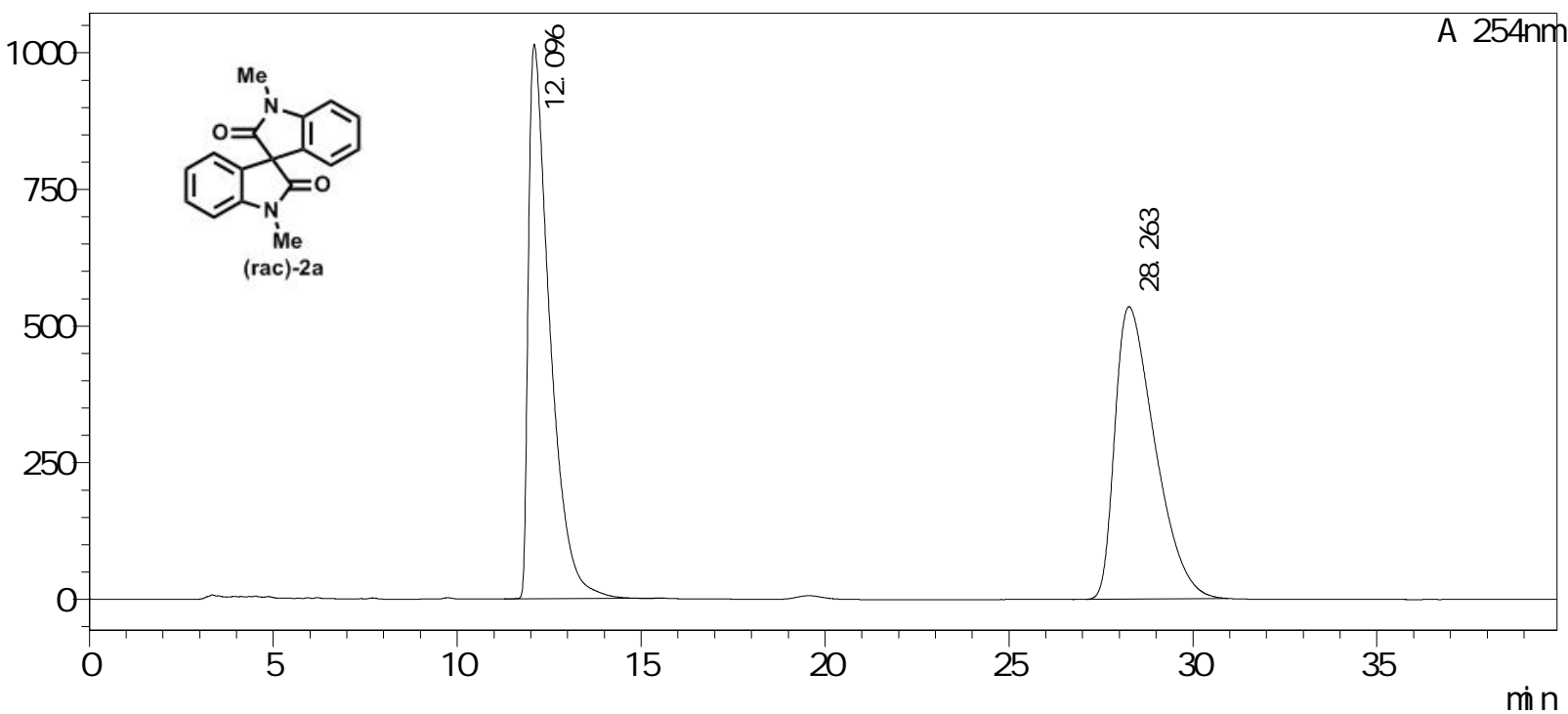

峰表>

检测器A $254 n m$

峰呚保卯时间

\begin{tabular}{|r|r|} 
峰号 & 保留时间 \\
\hline 1 & 12.096 \\
\hline 2 & 28.263 \\
\hline
\end{tabular}

面积 高度 1014518 浓度 浓度单位

标记

总计

50. 355 
 \\ LabSolutions 分析报告}

《样品信息>

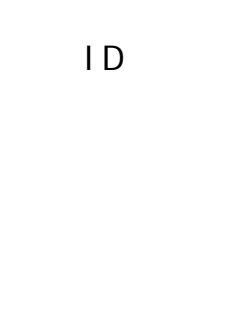

: CC

LT201801129- OD 30. I cd

OD H 30\% 60m n. I cm

1- 1

$1 \mathrm{uL}$

2018/12/12 15: 12: 27

样品类型：末知

2018/12/12 16: 18: 07

分析者 : Syst em Admi ni st $r$ at or

处理者 : Syst em Admi ni st $r$ at or

<色谱图>

$\mathrm{nV}$

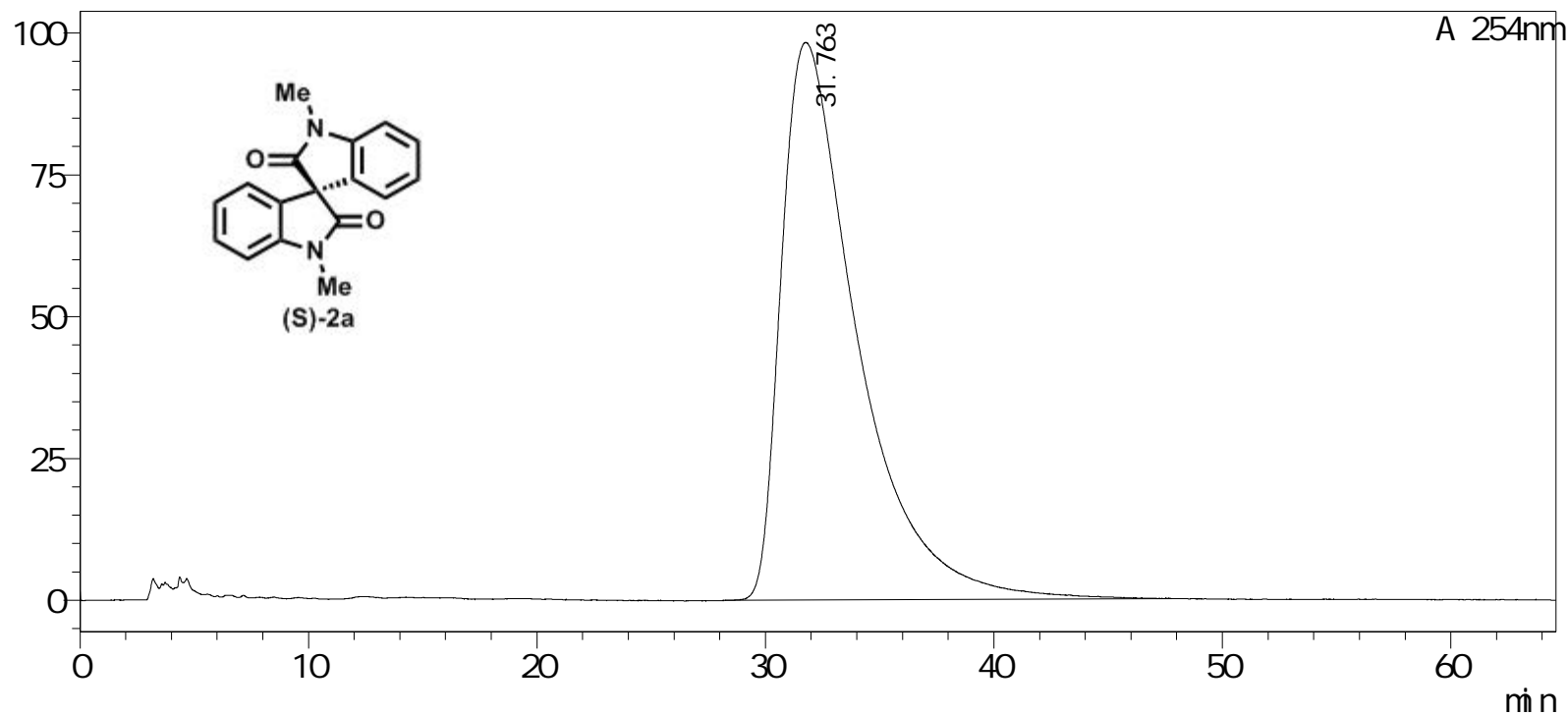

峰表>

检测器A $254 n m$

峰咢保卯时间

\begin{tabular}{r|r|}
\hline 1 & 31.763 \\
\hline
\end{tabular}

面积

22714491

9827

浓度 100.000 浓度单位

标记

98272 
 \\ LabSolutions 分析报告}

〈样品信息>

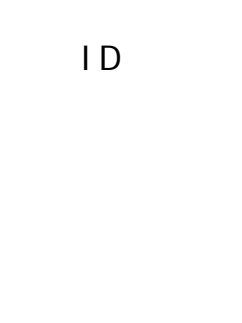

: CC

YI JI Bl AOO. I cd

OD H 30\% 60mi n. I cm

: $1-1$

$1 \mathrm{uL}$

2018/12/3 16: 19: 39

样品类型 ：未知

2018/12/3 16: 34: 40

分析者 : Syst em Admi ni st r at or

处理者 : Syst em Admi ni st $r$ at or

<色谱图>

nV

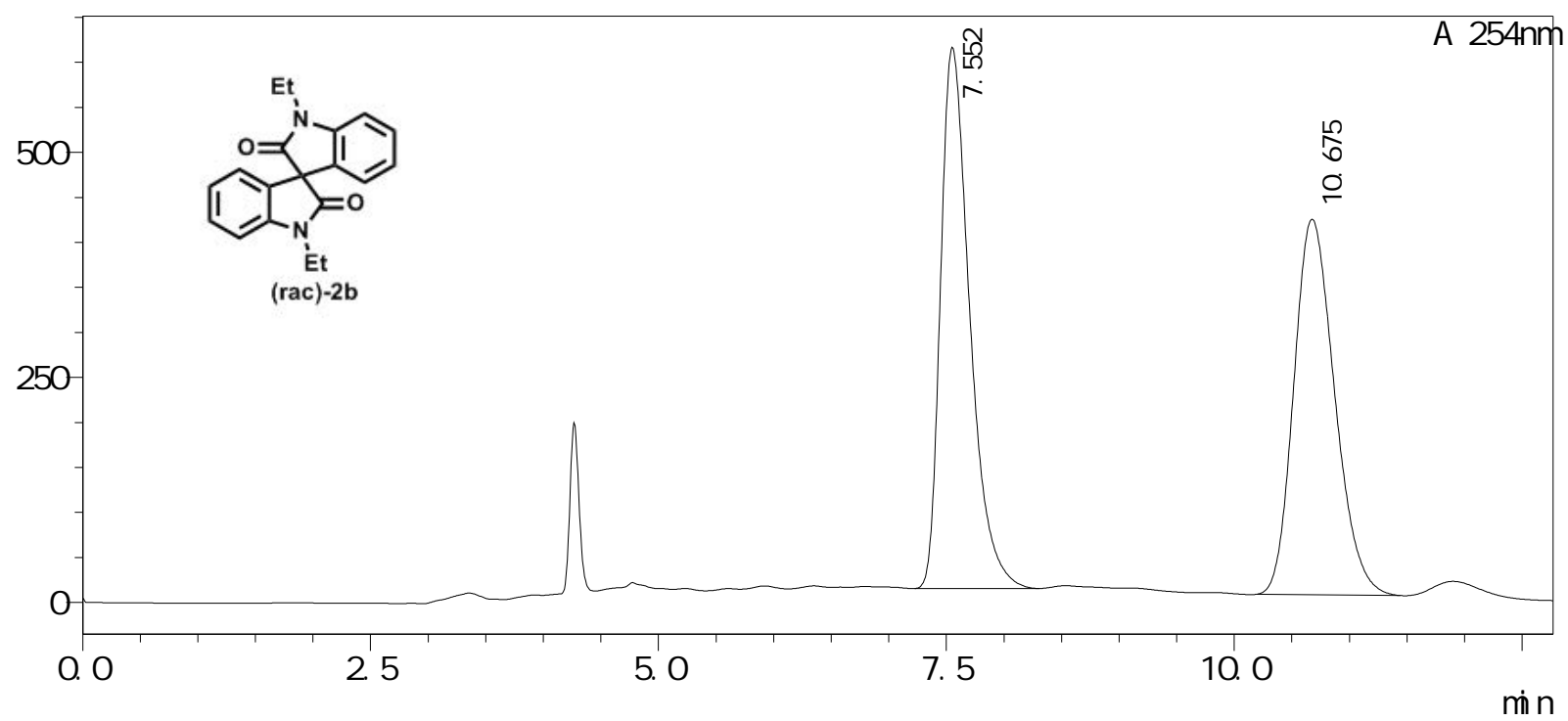

峰表>

检测器A $254 \mathrm{~nm}$

\begin{tabular}{|r|r|r|r|r|r|r|r|}
\hline 峰号 & 保留时间 & \multicolumn{1}{|c|}{ 面积 } & \multicolumn{1}{|c|}{ 高度 } & 浓度 & 浓度单位 & 标记 & 化合物名 \\
\hline 1 & 7.552 & 10586502 & 601246 & 50.785 & & $\mathrm{M}$ & \\
\hline 2 & 10.675 & 10259208 & 417248 & 49.215 & & $\mathrm{M}$ & \\
\hline 总计 & & 20845710 & 1018494 & & & & \\
\hline
\end{tabular}


 \\ LabSolutions 分析报告}

《样品信息>

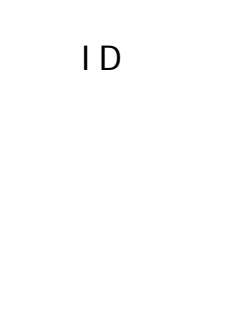

: CC

LT201801113 OD H 30\% I cd

OD H $30 \% 60 \mathrm{~min}$. I cm

1- 1

$1 \mathrm{uL}$

样品类型：未知

处理日期

2018/11/29 14: 58: 27

2018/11/29 19: $28: 08$

分析者

处理者

: Syst em Admi ni st $r$ at or

: Syst em Admi ni st $r$ at or

<色谱图>

nV

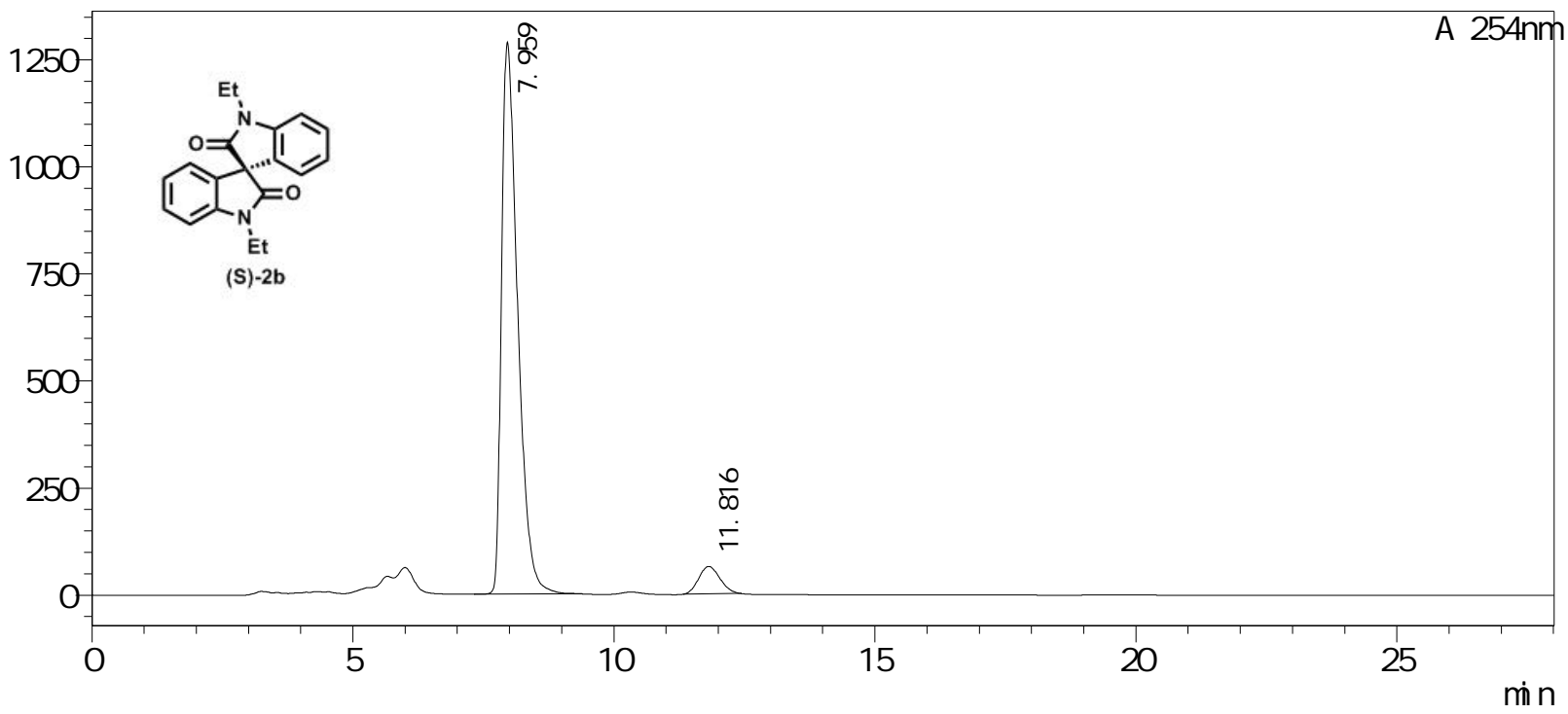

《峰表>

检测器A 254nm

|峰号 保留时间

\begin{tabular}{|r|r|}
\hline 1 & 7.959 \\
\hline 2 & 11.816 \\
\hline 总计
\end{tabular}

面积

高度

1812133

1289187

29516610

1353048

\begin{tabular}{r|r|r|r}
\multicolumn{1}{c|}{ 浓度 } & 浓度单位 & 标记 & 化合物名 \\
\hline 93.861 & & $\mathrm{M}$ & \\
\hline 6.139 & & $\mathrm{M}$ & \\
& & &
\end{tabular}


 \\ LabSolutions 分析报告}

《样品信息>

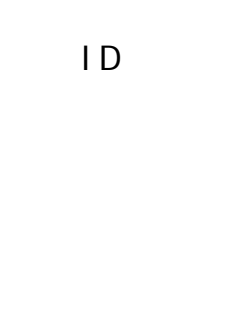

: CC

CC20181120-3 OD 30\% I cd

I C. H 30\% 60mi n. I cm

1- 1

$1 \mathrm{uL}$

2018/11/26 19: 14: 31

样品类型

: 未知

2018/11/29 19: 28: 07

分析者

: Syst em Admi ni st $r$ at or

处理者 $\quad$ Syst em Admi ni st $r$ at or

<色谱图>

$\mathrm{nV}$

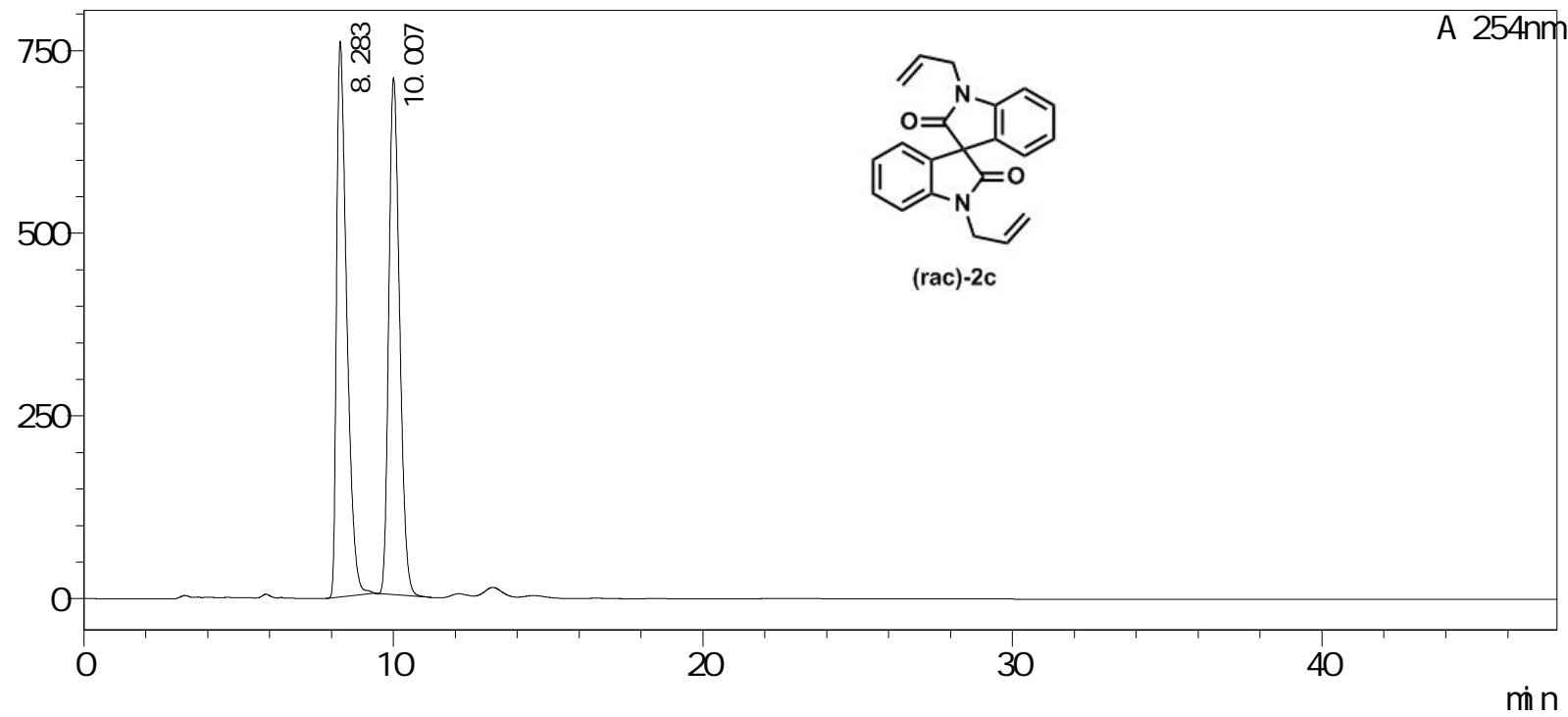

峰表>

检测器A $254 \mathrm{~nm}$

\begin{tabular}{|r|r|r|r|r|r|r|r|}
\hline 峰号 & 保留时间 & \multicolumn{1}{|c|}{ 面积 } & 高度 & 浓度 & 浓度单位 & 标记 & 化合物名 \\
\hline 1 & 8.283 & 17374664 & 759989 & 50.222 & & $\mathrm{M}$ & \\
\hline 2 & 10.007 & 17221138 & 707143 & 49.778 & & $\mathrm{M}$ & \\
\hline 总计 & & 34595802 & 1467132 & & & & \\
\hline
\end{tabular}


 \\ LabSolutions 分析报告}

《样品信息>

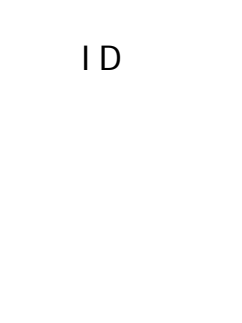

: $\mathrm{CC}$

LT201801122- OD 30. I cd

OD H 30\% 60mi n. I cm

1- 1

$1 \mathrm{uL}$

2018/12/2 16: 04: 08

样品类型：未知

2018/12/2 16: $34: 35$

分析者 : Syst em Admi ni st $r$ at or

处理者 : Syst em Admi ni st $r$ at or

<色谱图>

$\mathrm{nV}$

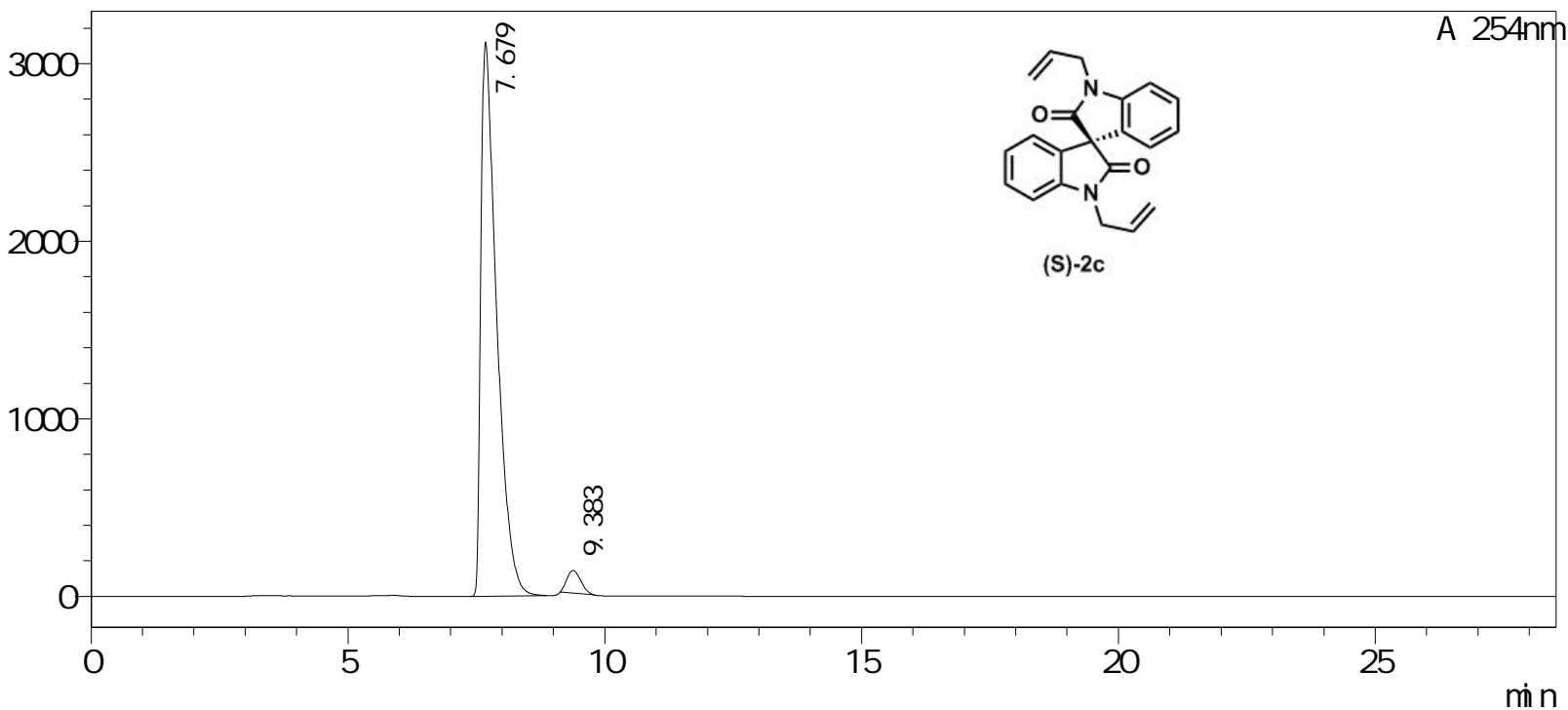

《峰表>

检测器A $254 n m$

\begin{tabular}{|r|r|r|r|r|r|r|r|}
\hline 峰号 & 保留时间 & \multicolumn{1}{|c|}{ 面积 } & 高度 & \multicolumn{1}{|c|}{ 浓度 } & 浓度单位 & 标记 & 化合物名 \\
\hline 1 & 7.679 & 67170385 & 3119235 & 96.517 & & $\mathrm{M}$ & \\
\hline 2 & 9.383 & 2423702 & 128415 & 3.483 & & $\mathrm{M}$ & \\
\hline 总计 & & 69594087 & 3247650 & & & & \\
\hline
\end{tabular}


 \\ LabSolutions 分析报告}

《样品信息>

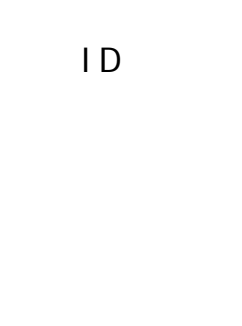

$:$ It

: CCBI ANI . 2-AS H 20. I cd

AS H $20 \% 100 \mathrm{mi}$. I cm

: $1-1$

$1 \mathrm{uL}$

2018/12/20 9: 30: 45

2018/12/20 10:04:02

样品类型：未知

分析者 : Syst em Admi ni st r at or

处理者 $\quad$ Syst em Admi ni st $r$ at or

<色谱图>

nV

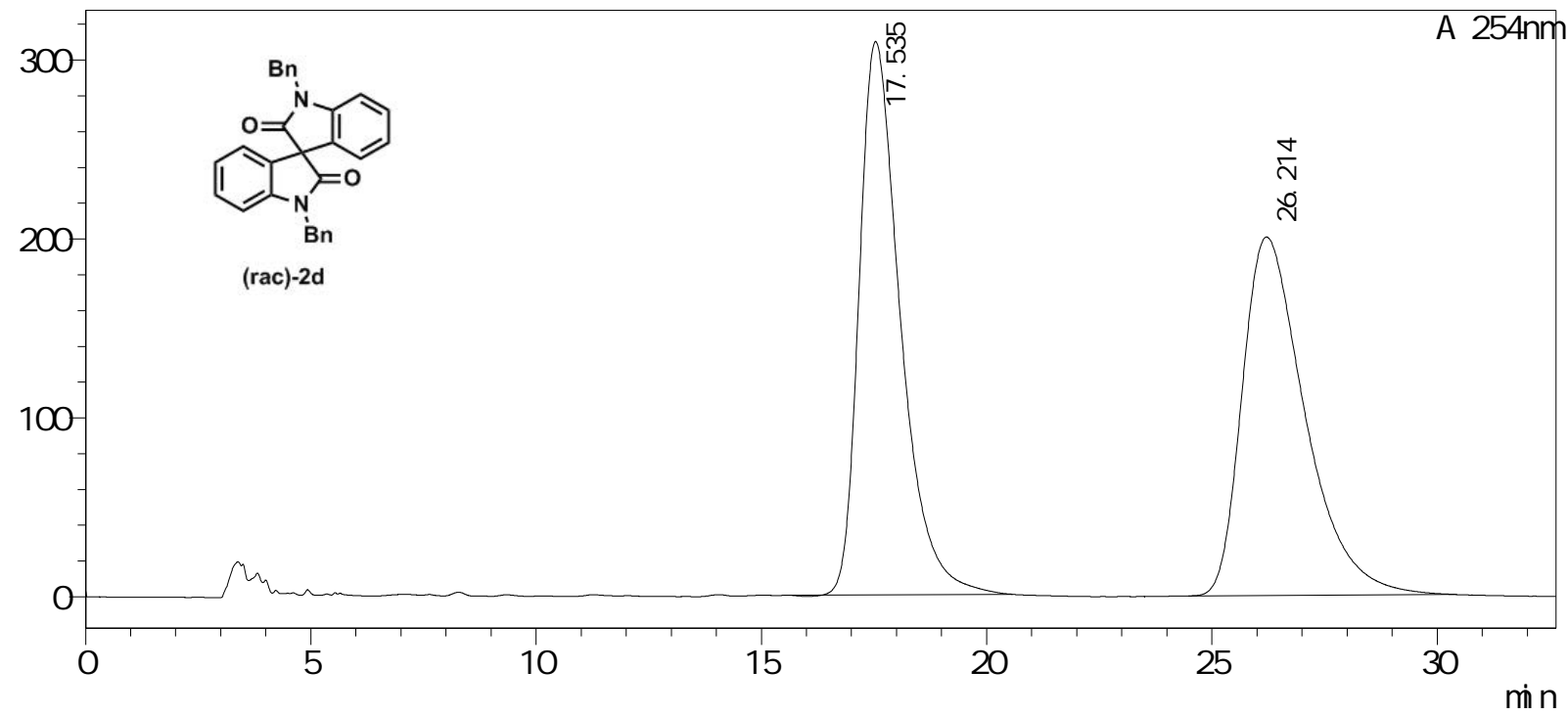

峰表>

检测器 $\mathrm{A} 254 \mathrm{~nm}$

峰咢 保留时间

\begin{tabular}{|r|r|}
\hline 1 & 17.535 \\
\hline 2 & 26.214 \\
\hline
\end{tabular}

面积

19651183

19419401

39070584

\begin{tabular}{|l|}
\hline 高度 \\
\hline 309314 \\
\hline 200364 \\
\hline 509678 \\
\hline
\end{tabular}

浓度

50.297

49.703

浓度单位

标记

总计 
 \\ LabSolutions 分析报告}

《样品信息>

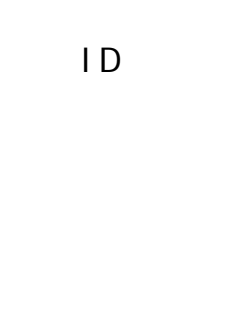

$:$ It

: LT201801145-AS H 20. I cd

AS H $20 \% 100 \mathrm{mi}$. I cm

1- 1

$1 \mathrm{uL}$

2018/12/19 21:53: 50

样品类型：未知

: 2018/12/21 11: $45: 56$

分析者

- Syst em Admi ni st r at or

处理者 : Syst em Admi ni st $r$ at or

<色谱图>

nV

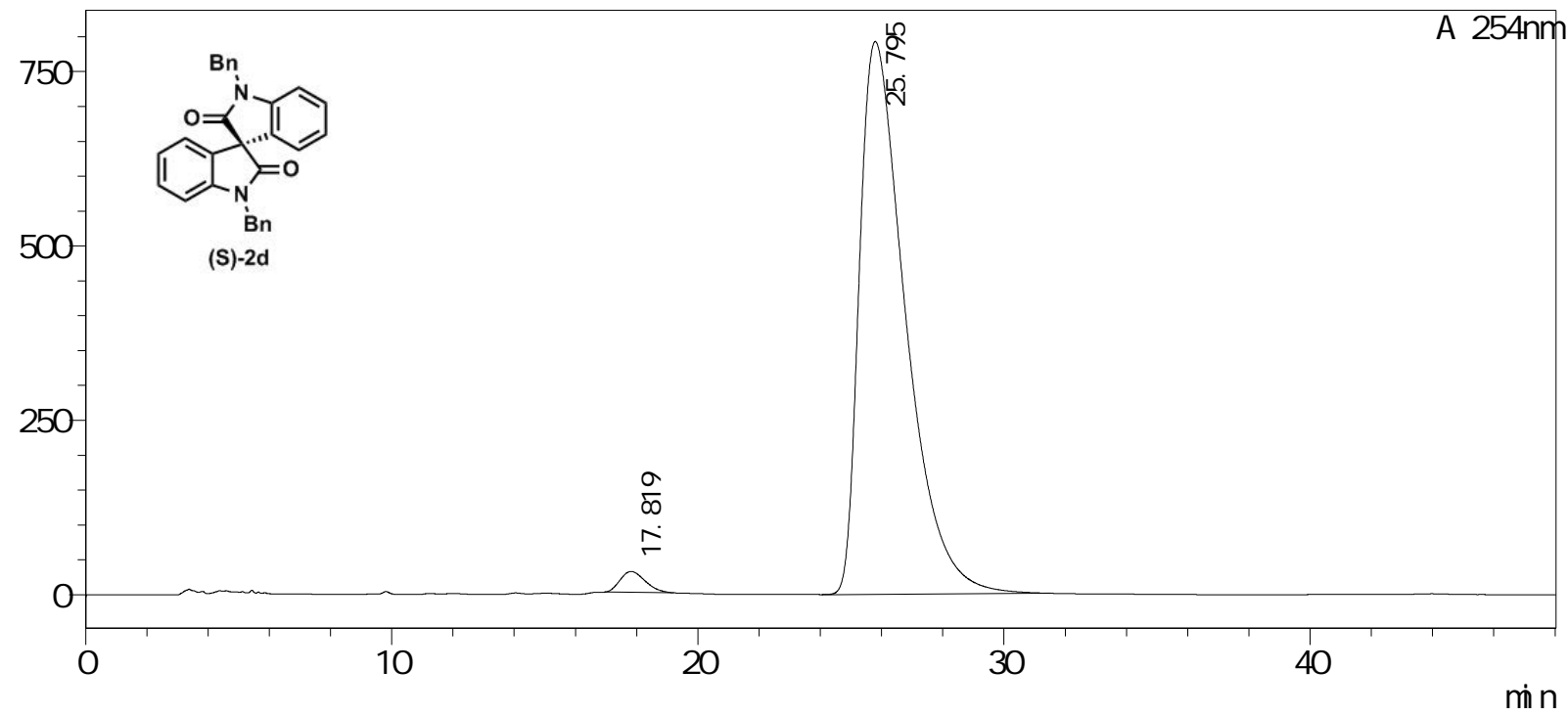

峰表>

检测器A $254 \mathrm{~nm}$

\begin{tabular}{|r|r|r|r|r|r|r|r|}
\hline 峰号 & 保留时间 & \multicolumn{1}{|c|}{ 面积 } & \multicolumn{1}{|c|}{ 高度 } & 浓度 & 浓度单位 & 标记 & 化合物名 \\
\hline 1 & 17.819 & 1712946 & 29973 & 2.025 & & $\mathrm{M}$ & \\
\hline 2 & 25.795 & 82859084 & 792647 & 97.975 & & $\mathrm{M}$ & \\
\hline 总计 & & 84572030 & 822620 & & & & \\
\hline
\end{tabular}


 \\ LabSolutions 分析报告}

《样品信息>

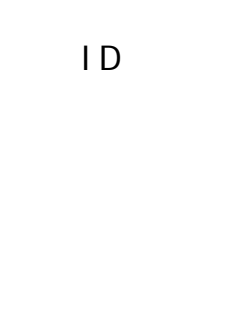

: $\mathrm{CC}$

: CC20181118- 2- OD H 15\% I cd

OD H $15 \%$ 40mi n. I cm

: $1-1$

$1 \mathrm{uL}$

样品类型：未知

处理日期

2018/11/30 14: $56: 51$

2018/12/1 11: 24: 27

分析者

处理者

: Syst em Admi ni st r at or

: Syst em Admi ni st $r$ at or

<色谱图>

$\mathrm{nV}$

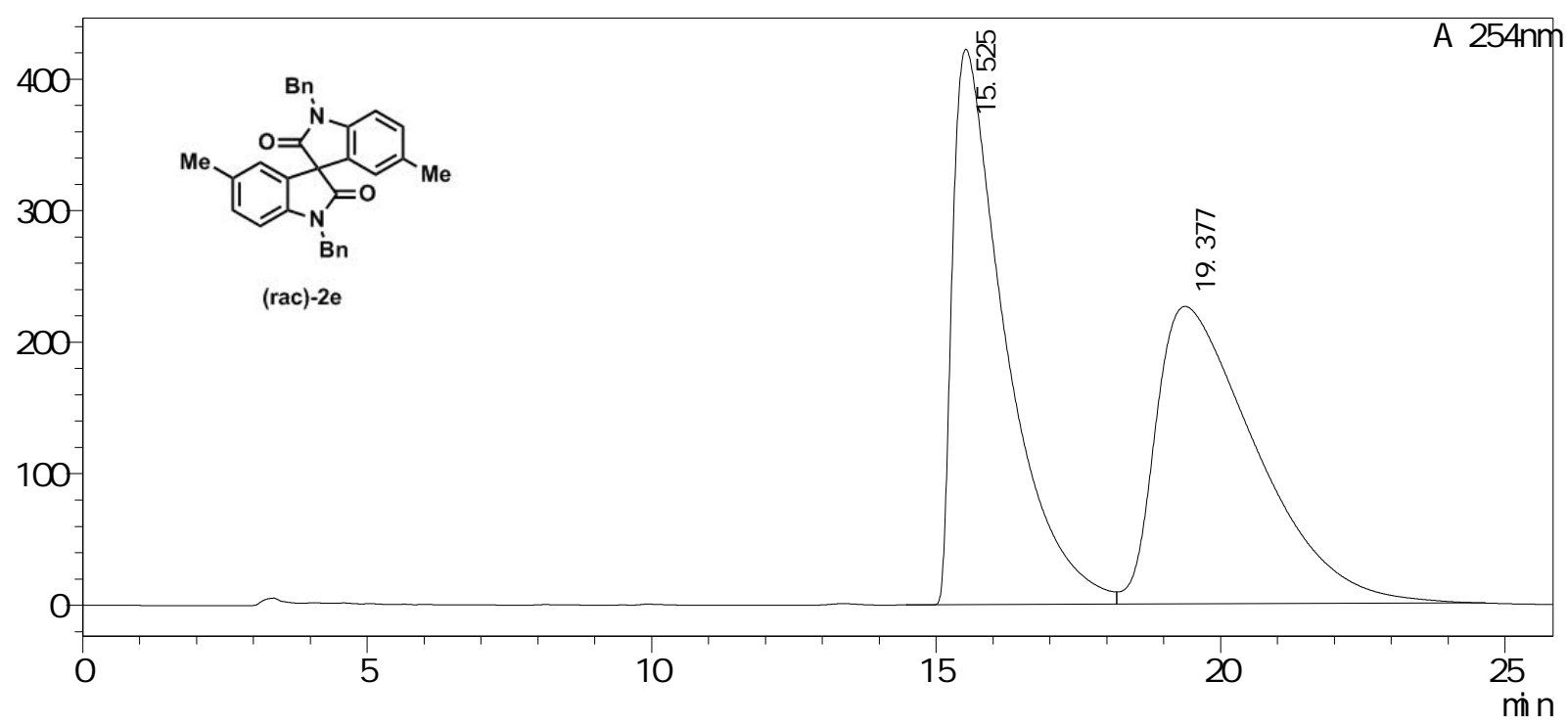

峰表>

检测器 $\mathrm{A} 254 \mathrm{~nm}$

\begin{tabular}{|r|r|r|r|r|r|r|r|}
\hline 峰号 & 保留时间 & 面积 & 高度 & 浓度 & 浓度单位 & 标记 & 化合物名 \\
\hline 1 & 15.525 & 27410566 & 422338 & 49.649 & & $\mathrm{M}$ & \\
\hline 2 & 19.377 & 27798470 & 226267 & 50.351 & & $\mathrm{~V} \mathrm{M}$ & \\
\hline 总计 & & 55209037 & 648605 & & & & \\
\hline
\end{tabular}


 \\ LabSolutions 分析报告}

《样品信息>

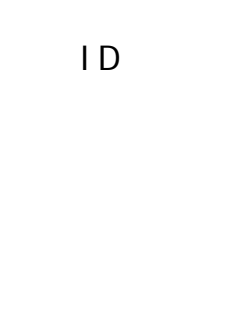

: CC

LT201801128 OD 15\% I cd

OD H $15 \%$ 40mi n. I cm

1- 1

$1 \mathrm{uL}$

2018/12/6 16: 01: 27

样品类型：未知

处理日期

: 2018/12/7 9: 02: 16

分析者 : Syst em Admi ni st r at or

处理者 : Syst em Admi ni st $r$ at or

<色谱图>

$\mathrm{nV}$

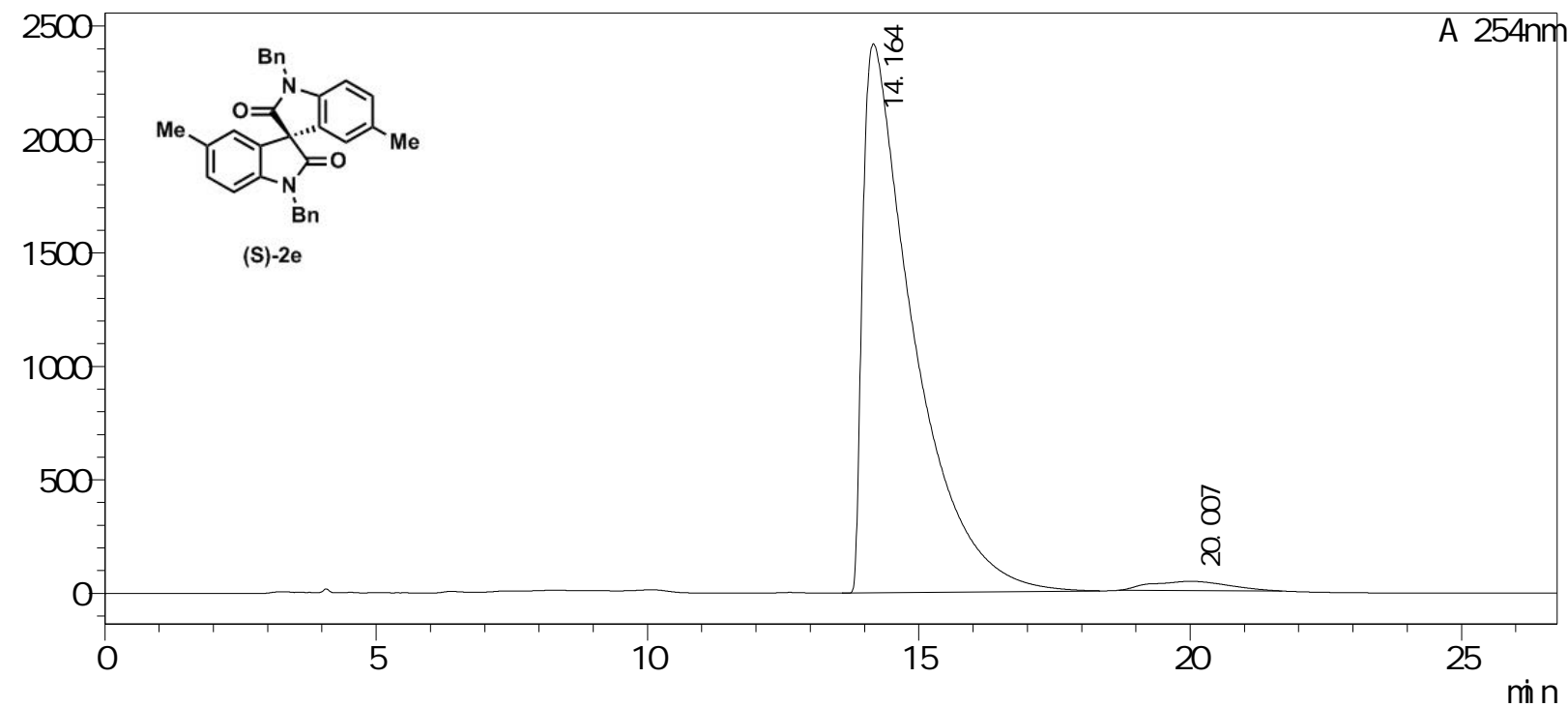

《峰表>

检测器A $254 \mathrm{~nm}$

\begin{tabular}{|r|r|r|r|r|r|r|r|}
\hline 峰号 & 保留时间 & \multicolumn{1}{|c|}{ 面积 } & \multicolumn{1}{|c|}{ 高度 } & \multicolumn{1}{|c|}{ 浓度 } & 浓度单位 & 标记 & 化合物名 \\
\hline 1 & 14.164 & 157604482 & 2420115 & 97.482 & & $\mathrm{M}$ & \\
\hline 2 & 20.007 & 4071082 & 40641 & 2.518 & & $\mathrm{M}$ & \\
\hline 总计 & & 161675563 & 2460756 & & & & \\
\hline
\end{tabular}


 \\ LabSolutions 分析报告}

《样品信息>

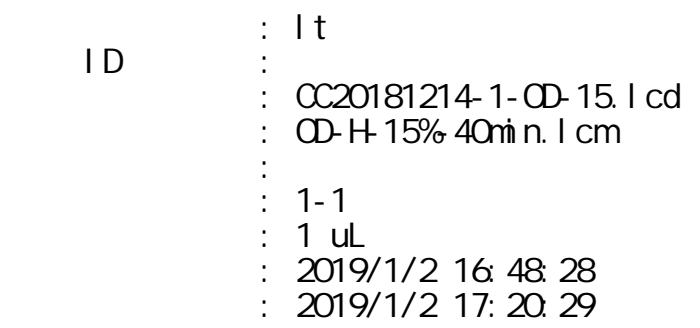

样品类型 ：未知

分析者 : Syst em Admi ni st $r$ at or

处理者 : Syst em Admi ni st $r$ at or

<色谱图>

$\mathrm{nV}$

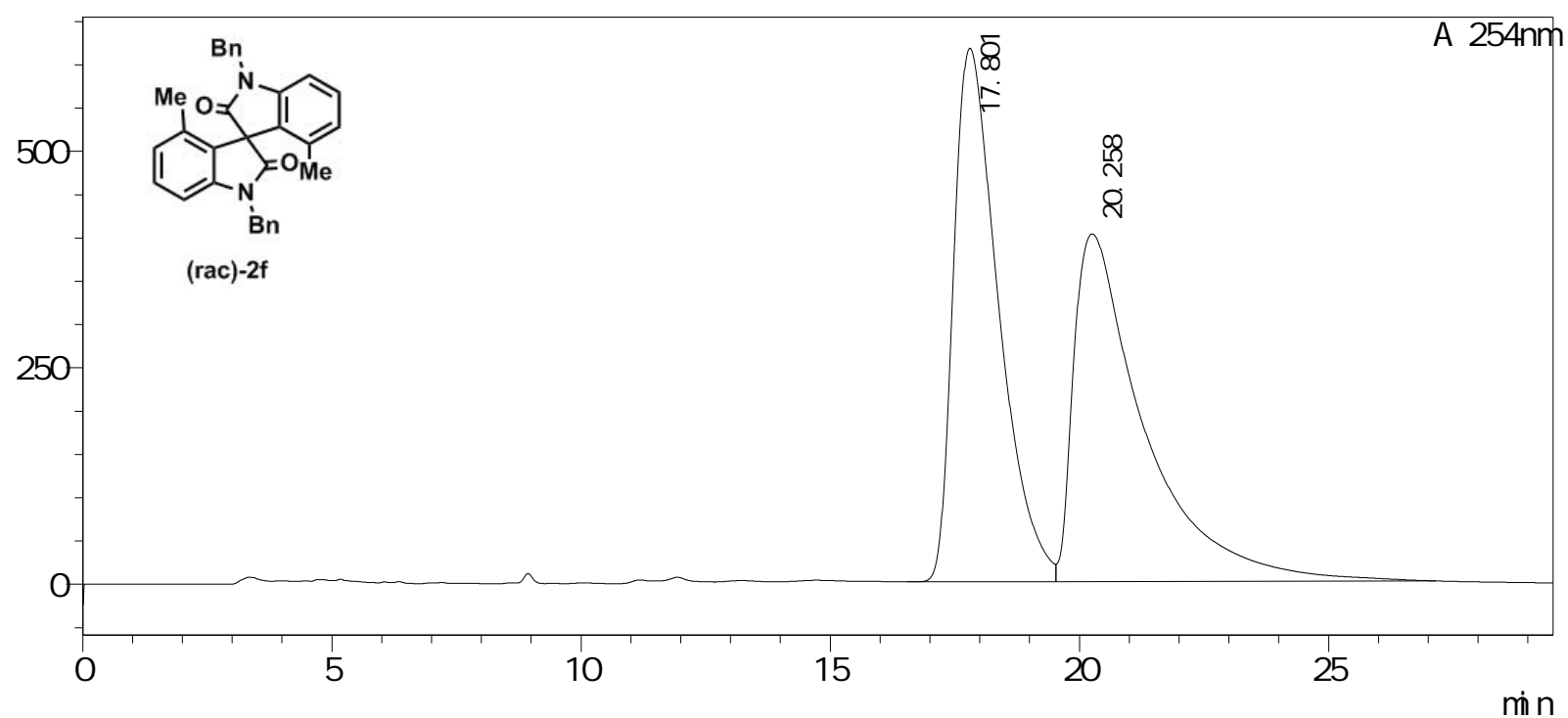

峰表>

检测器A $254 \mathrm{~nm}$

\begin{tabular}{|r|r|r|r|r|r|r|r|}
\hline 峰号 & 保留时间 & \multicolumn{1}{|c|}{ 面积 } & \multicolumn{1}{|c|}{ 度 } & 浓度 & 浓度单位 & 标记 & 化合物名 \\
\hline 1 & 17.801 & 38706407 & 616197 & 49.355 & & $\mathrm{M}$ & \\
\hline 2 & 20.258 & 39718341 & 401550 & 50.645 & & $\mathrm{~V} \mathrm{M}$ & \\
\hline 总计 & & 78424748 & 1017746 & & & & \\
\hline
\end{tabular}


 \\ LabSolutions 分析报告}

《样品信息>

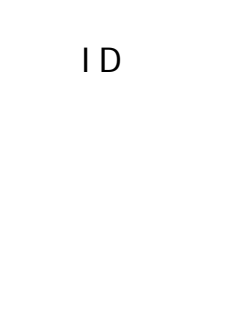

$:$ It

: LT201802029- OD 15. I cd

: OD H 15\% 40mi n. I cm

: $1-1$

$1 \mathrm{uL}$

2018/12/31 10: 36: 16

样品类型：未知

处理日期

: 2019/1/2 17: 05: 31

分析者 : Syst em Admi ni st r at or

处理者 : Syst em Admi ni st $r$ at or

<色谱图>

nV

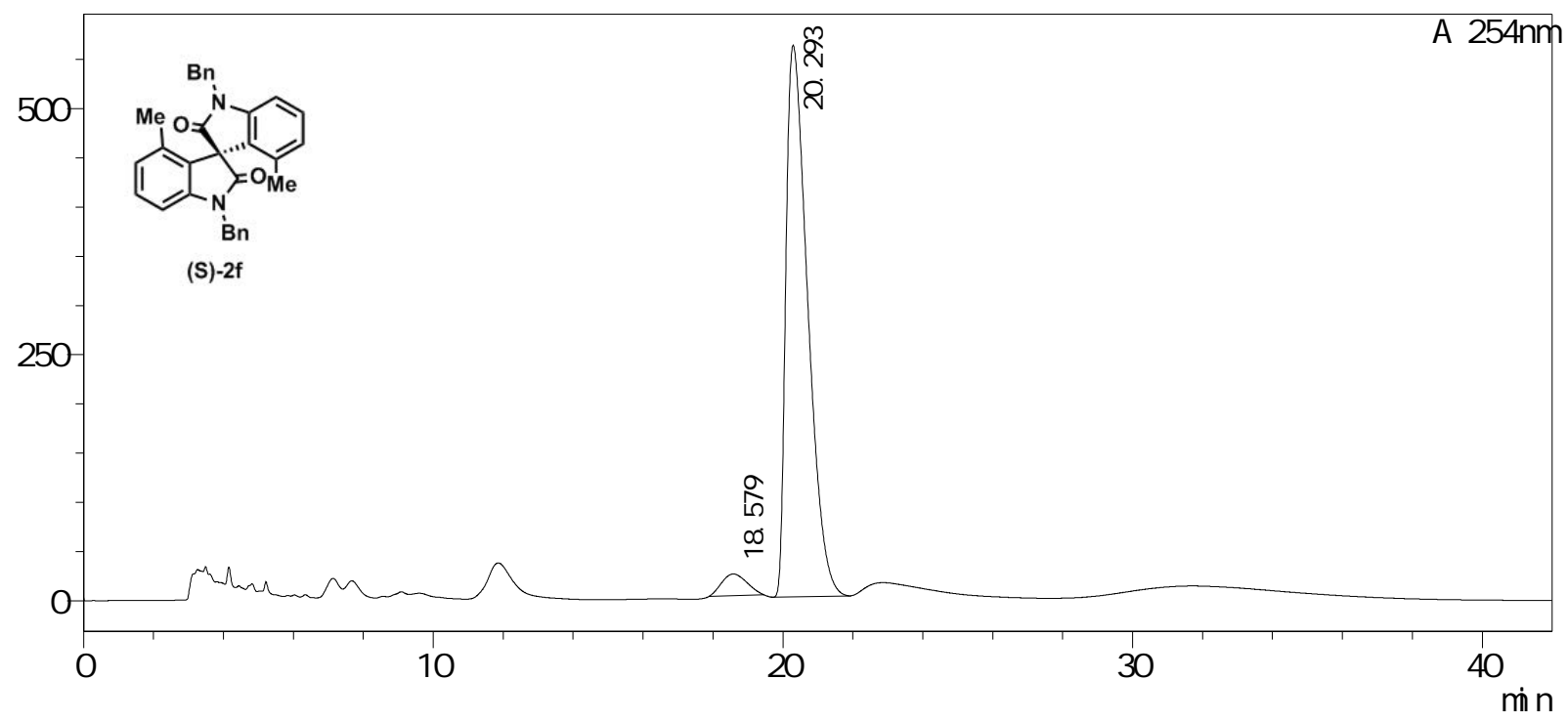

《峰表>

检测器A $254 \mathrm{~nm}$

\begin{tabular}{|r|r|r|r|r|r|r|r|}
\hline 峰号 & 保留时间 & 面积 & \multicolumn{1}{|c|}{ 高度 } & \multicolumn{1}{|c|}{ 浓度 } & 浓度单位 & 标记 & 化合物名 \\
\hline 1 & 18.579 & 1093389 & 21918 & 4.318 & & $\mathrm{M}$ & \\
\hline 2 & 20.293 & 24225369 & 560456 & 95.682 & & $\mathrm{M}$ & \\
\hline 总计 & & 25318758 & 582374 & & & & \\
\hline
\end{tabular}


 \\ LabSolutions 分析报告}

《样品信息>

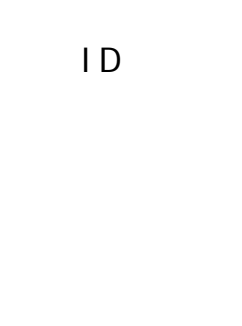

: It

: LT201802031BI AO. 2- AS H 20. I cd AS H $20 \% 100 \mathrm{mi}$. I cm

1- 1

$1 \mathrm{uL}$

2019/1/3 17: 23: 36

2019/1/3 22: 09: 19
样品类型 ：未知

分析者 : Syst em Admi ni st $r$ at or

处理者 $\quad$ Syst em Admi ni st $r$ at or

<色谱图>

nV

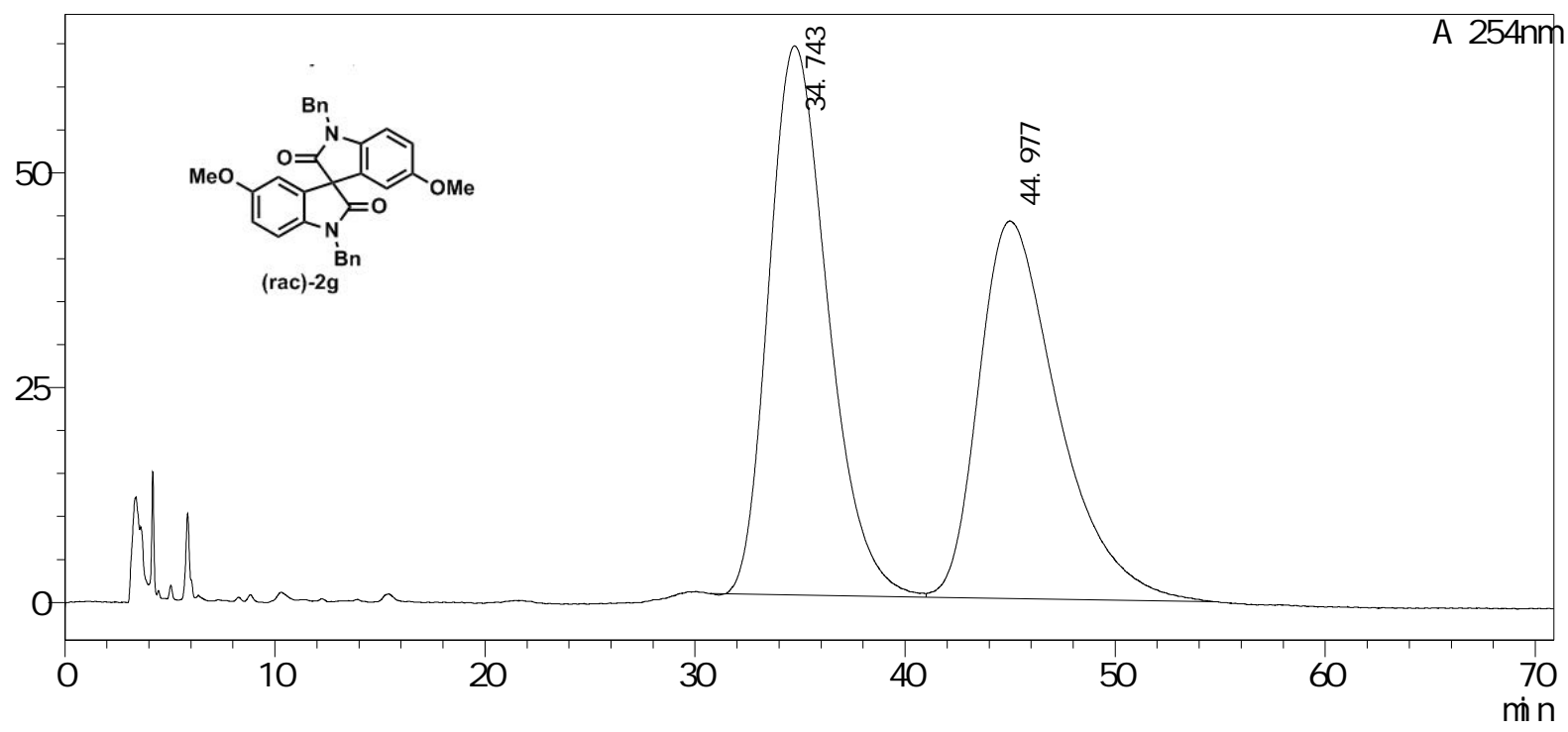

《峰表>

检测器 $\mathrm{A} 254 \mathrm{~nm}$

峰咢 保留时间

\begin{tabular}{|r|}
\hline 1 \\
\hline 2
\end{tabular}

34.743

44. 977

面积

12500421

高度

24018906

63907

浓度 浓度单位

标记


 \\ LabSolutions 分析报告}

《样品信息>

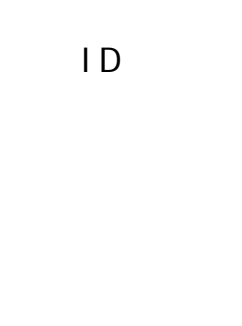

: It

: LT201802030-AS H 20. I cd

AS H $20 \% 100 \mathrm{mi}$. I cm

1- 1

$1 \mathrm{uL}$

2019/1/3 16: 17: 25

样品类型：未知

处理日期

: 2019/1/3 22: 09: 21

分析者 : Syst em Admi ni st r at or

处理者 : Syst em Admi ni st $r$ at or

<色谱图>

nV

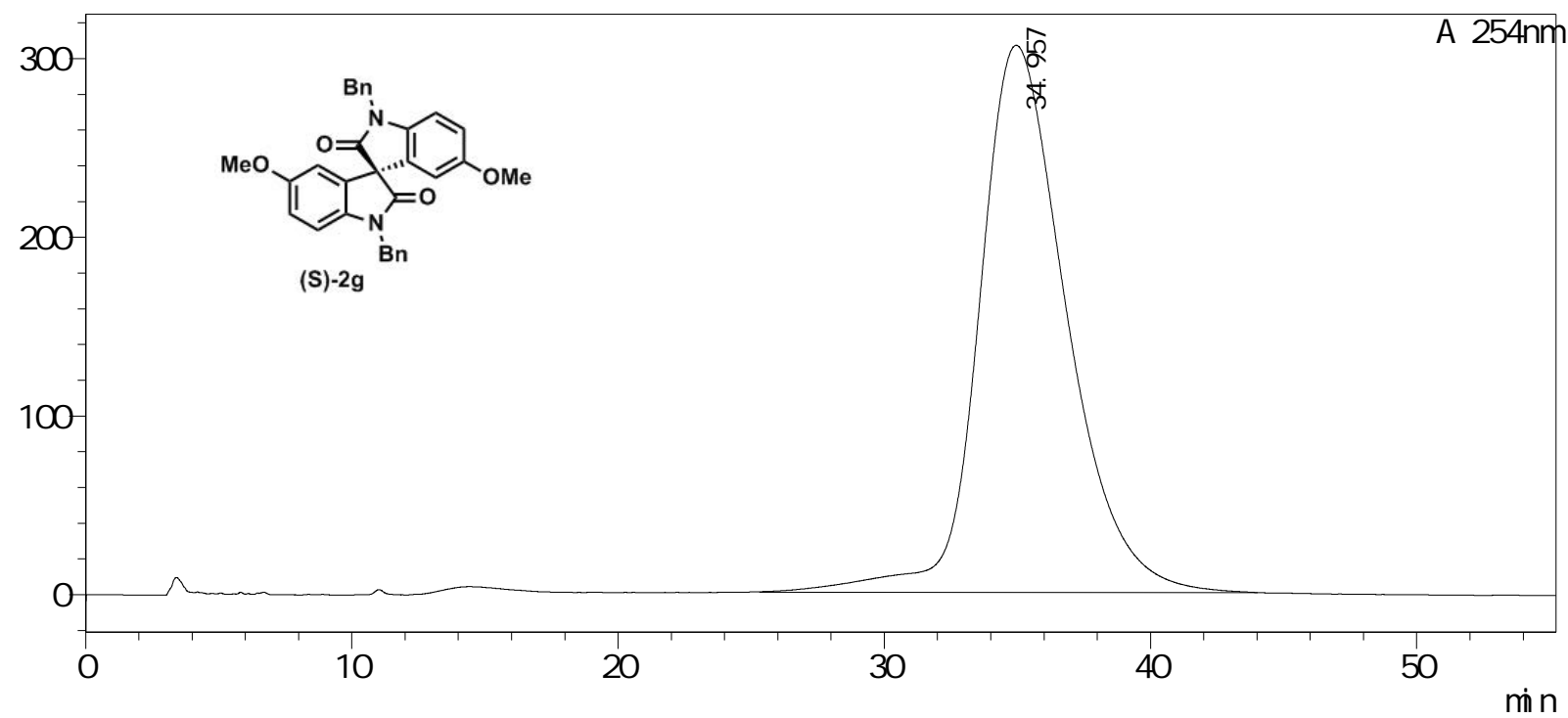

峰表>

检测器A $254 \mathrm{~nm}$

峰骂保留时间

\begin{tabular}{r|r|}
\hline 1 & 34.957 \\
\hline
\end{tabular}

34. 957 面积

总计

70113281

高度
306220
306220

浓度 100.000 浓度单位 
 \\ LabSolutions 分析报告}

《样品信息>

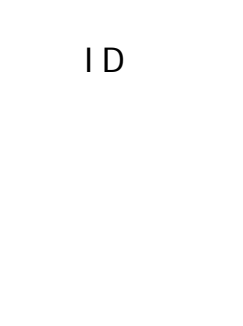

: It

CC20181118 1. 3 AS H 20. I cd

AS $\mathrm{H} 20 \%$ 100m n. I cm

1- 1

$1 \mathrm{uL}$

样品类型 ：未知

处理日期

2018/12/21 14: $22: 57$

2018/12/21 15: 43: 01

分析者

处理者

: Syst em Admi ni st r at or

: Syst em Admi ni st $r$ at or

<色谱图>

$\mathrm{nV}$

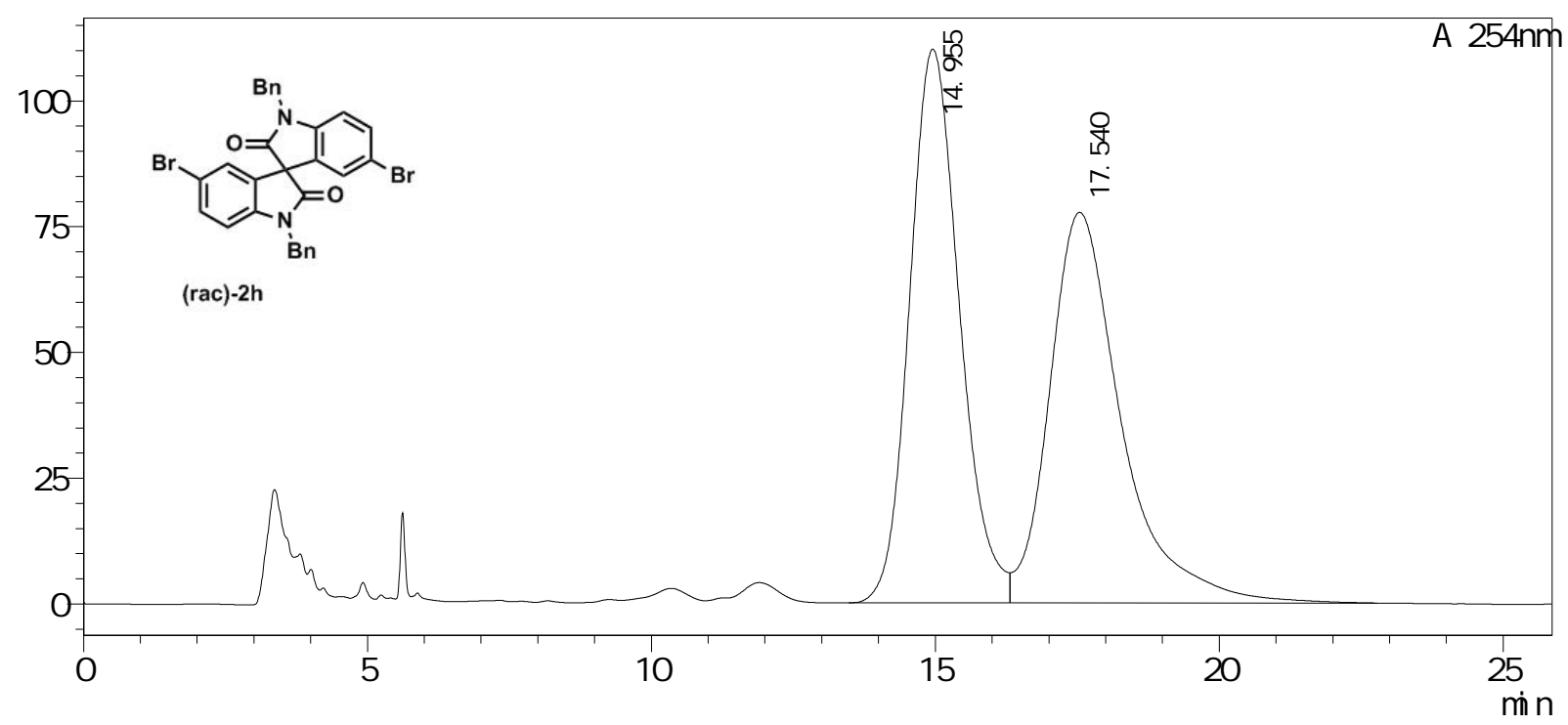

凶峰表>

检测器A $254 \mathrm{~nm}$

检洛器 $254 n$

\begin{tabular}{|r|r|r|r|r|r|r|r|}
\hline 峰号 & 保留时间 & \multicolumn{1}{|c|}{ 面积 } & \multicolumn{1}{|c|}{ 高度 } & 浓度 & 浓度单位 & 标记 & 化合物名 \\
\hline 1 & 14.955 & 6743004 & 110103 & 49.692 & & $\mathrm{~V}$ & \\
\hline 2 & 17.540 & 6826542 & 77681 & 50.308 & & $\mathrm{SV}$ & \\
\hline 总计 & & 13569546 & 187785 & & & & \\
\hline
\end{tabular}


 \\ LabSolutions 分析报告}

《样品信息>

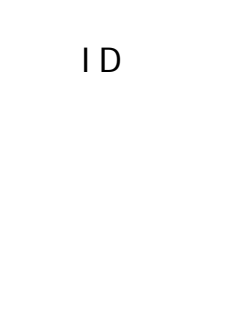

$:$ It

: LT201801117- AS- H 20. I cd AS H $20 \% 100 \mathrm{mi}$. I cm

1- 1

$1 \mathrm{uL}$

2018/12/21 11: 19: 49

样品类型 ：未知

: 2018/12/21 15: 43: 37

分析者 : Syst em Admi ni st $r$ at or

处理者 : Syst em Admi ni st $r$ at or

<色谱图>

nV

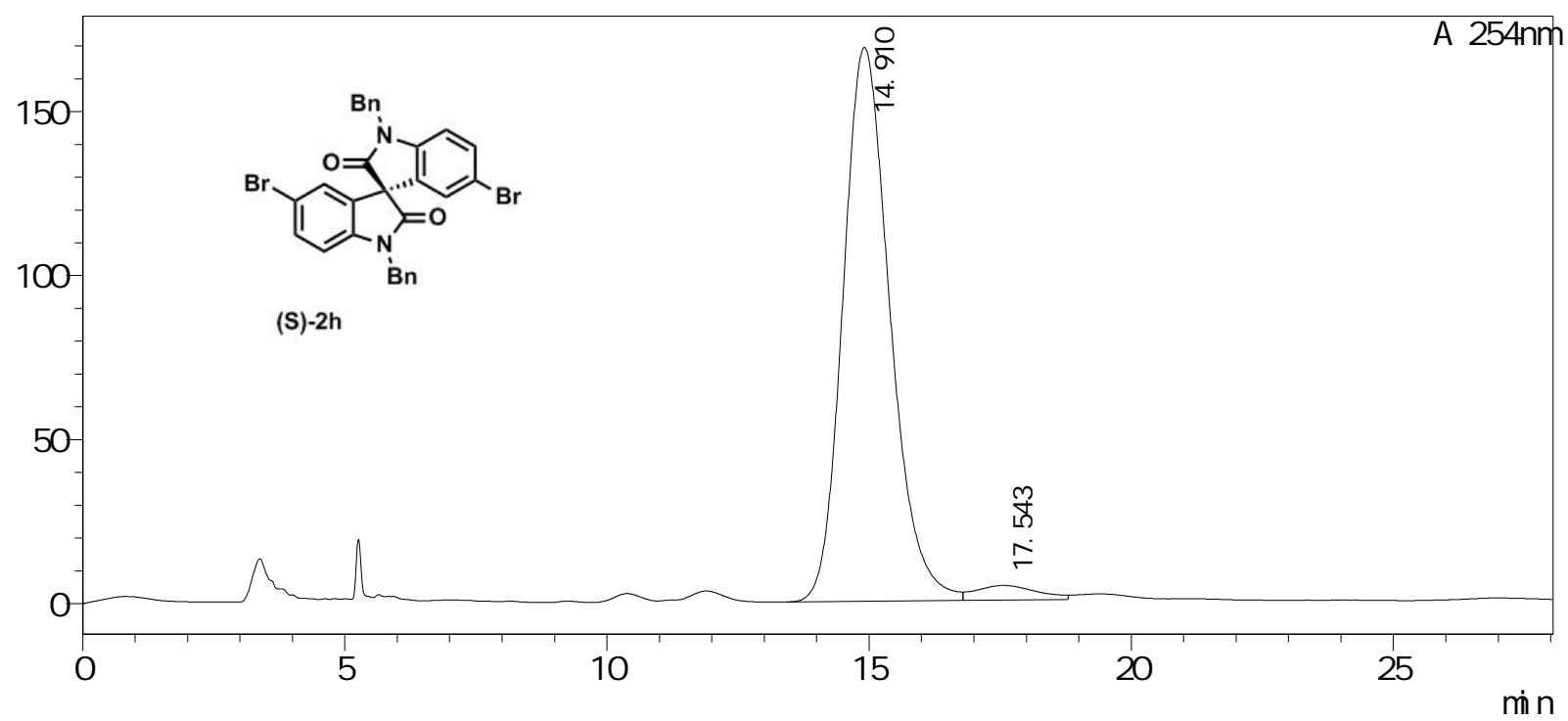

凶峰表>

检测器A $254 \mathrm{~nm}$

峰咢保留时间

\begin{tabular}{|r|r|}
\hline 1 & 14.910 \\
\hline 2 & 17.543 \\
\hline
\end{tabular}

面积

17.543

10521016
372233

10893249

高度

4510

173430

浓度

96.583 浓度单位

标记

总计 
 \\ LabSolutions 分析报告}

《样品信息>

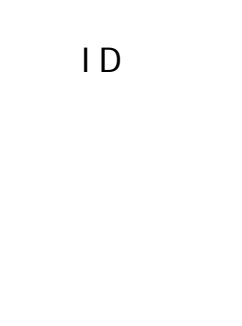

: It

: cc20181129-3- oj - 20. I cd

OJ - H $20 \% 60 \mathrm{mi} \mathrm{n.} \mathrm{I} \mathrm{cm}$

: $1-1$

$1 \mathrm{uL}$

2018/12/22 10: 49: 14

样品类型：未知

: 2018/12/22 11: 21: 01

分析者 : Syst em Admi ni st r at or

处理者 : Syst em Admi ni st $r$ at or

<色谱图>

$\mathrm{nV}$

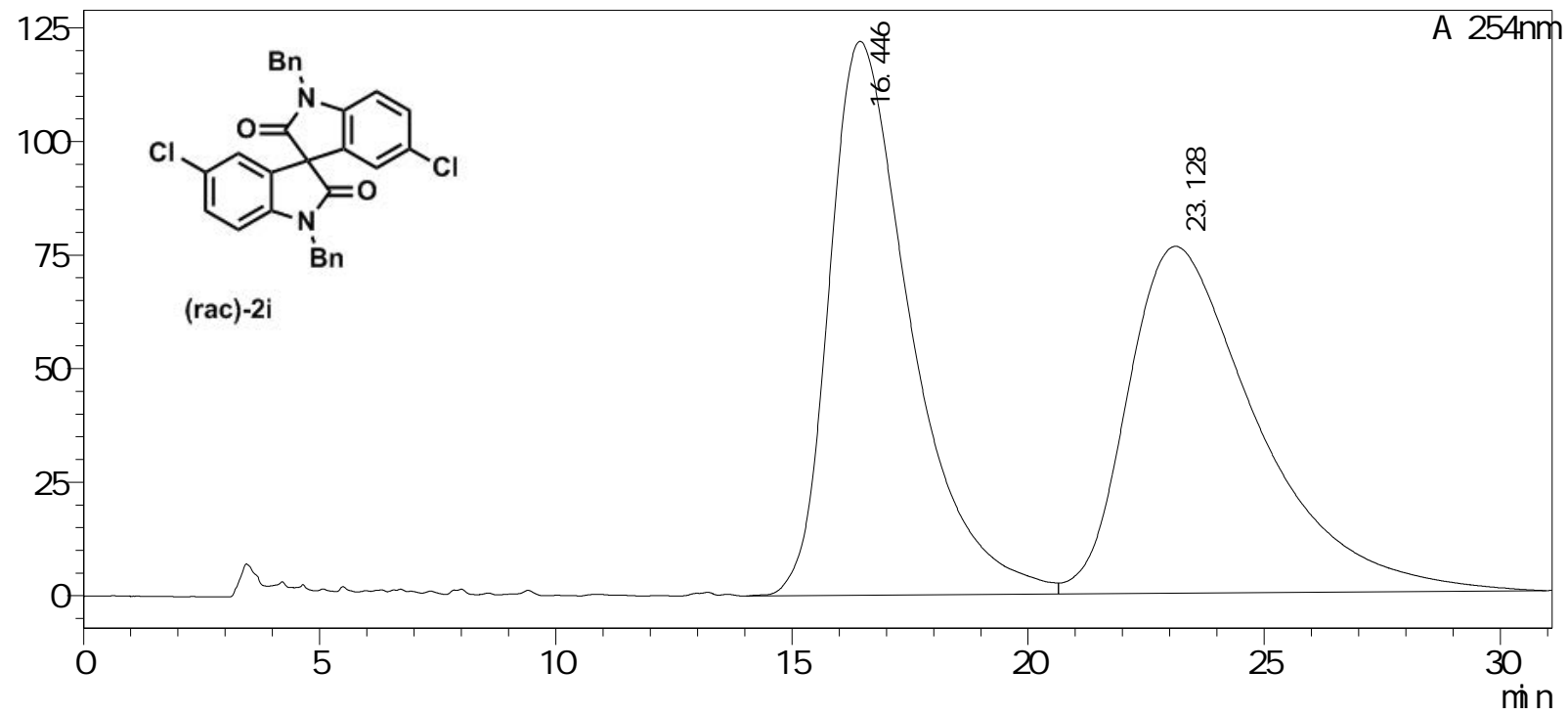

峰表>

检测器A $254 \mathrm{~nm}$

\begin{tabular}{|r|r|r|r|r|l|l|l|}
\hline 峰号 & 保留时间 & \multicolumn{1}{|c|}{ 面积 } & 高度 & 浓度 & 浓度单位 & 标记 & 化合物名 \\
\hline 1 & 16.446 & 14644415 & 121925 & 50.059 & & & \\
\hline 2 & 23.128 & 14609844 & 76353 & 49.941 & & $\mathrm{~V}$ & \\
\hline 总计 & & 29254259 & 198278 & & & & \\
\hline
\end{tabular}


 \\ LabSolutions 分析报告}

《样品信息>

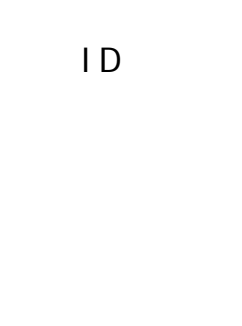

$:$ It

LT201801144 OJ-20. I cd

OJ - H $20 \%$ 60mi n. I cm

1- 1

$1 \mathrm{uL}$

2018/12/18 18: 54: 53

样品类型：未知

: 2018/12/18 21: 21: 48

分析者 : Syst em Admi ni st r at or

处理者 : Syst em Admi ni st $r$ at or

<色谱图>

$\mathrm{nV}$

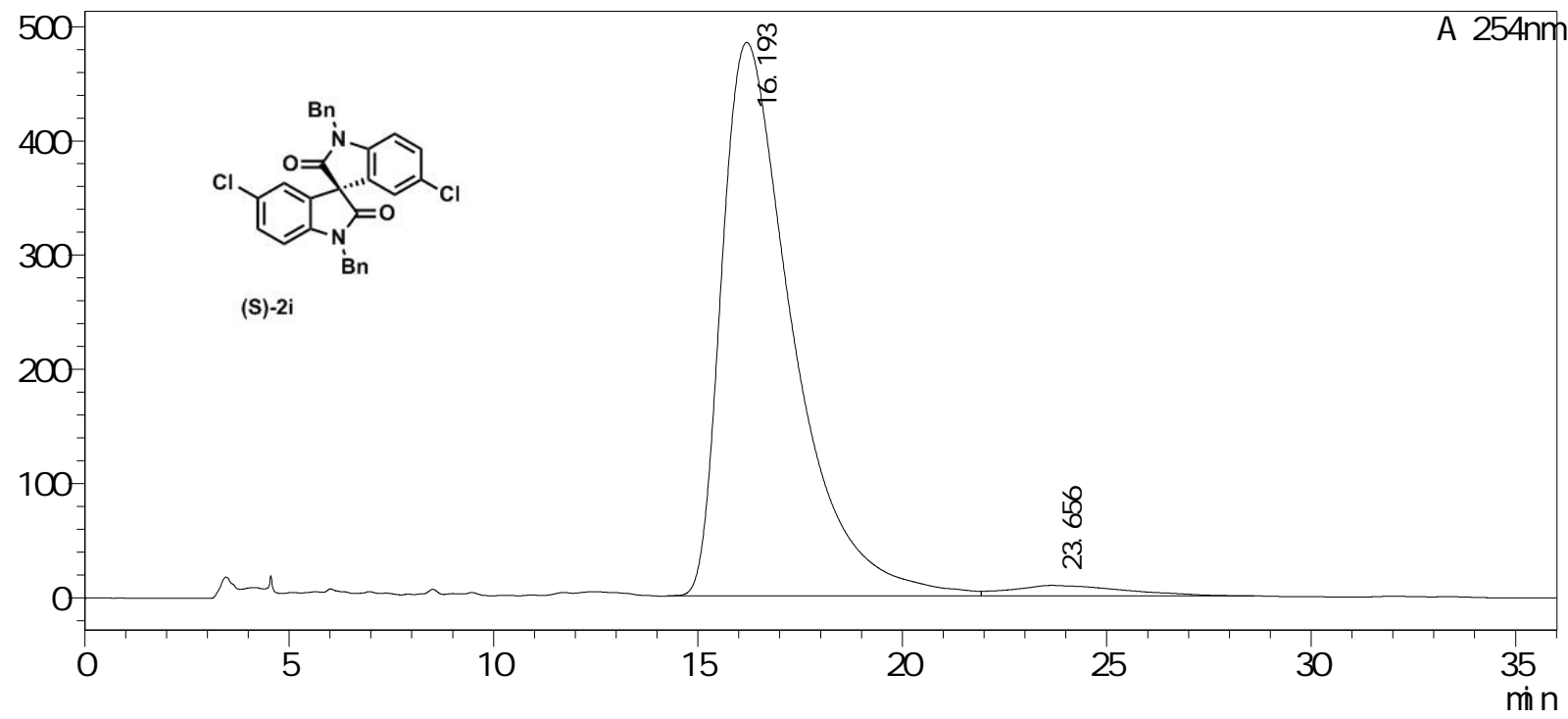

峰表>

检测器 $\mathrm{A} 254 \mathrm{~nm}$

峰咢保留时间

\begin{tabular}{|r|r|}
\hline 1 & 16.193 \\
\hline 2 & 23.656 \\
\hline
\end{tabular}

面积

58450797

高度

1752026

60202823

484355

浓度

97.090 浓度单位

\begin{tabular}{|r|r}
\hline 标记 & 化合物名 \\
\hline $\mathrm{VM}$ & \\
\hline
\end{tabular}


 \\ LabSolutions 分析报告}

《样品信息>

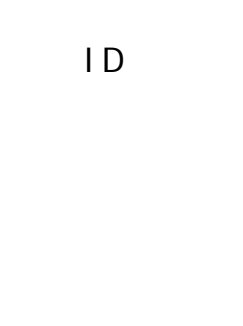

: It

: CCZH JI 1. 21- OD H 40. I cd

OD H 40\% 100mi n. I cm

: $1-1$

$1 \mathrm{uL}$

2019/1/21 9: 51: 04

样品类型：未知

2019/1/21 10: 29: 10

分析者 : Syst em Admi ni st $r$ at or

处理者 : Syst em Admi ni st $r$ at or

<色谱图>

nV

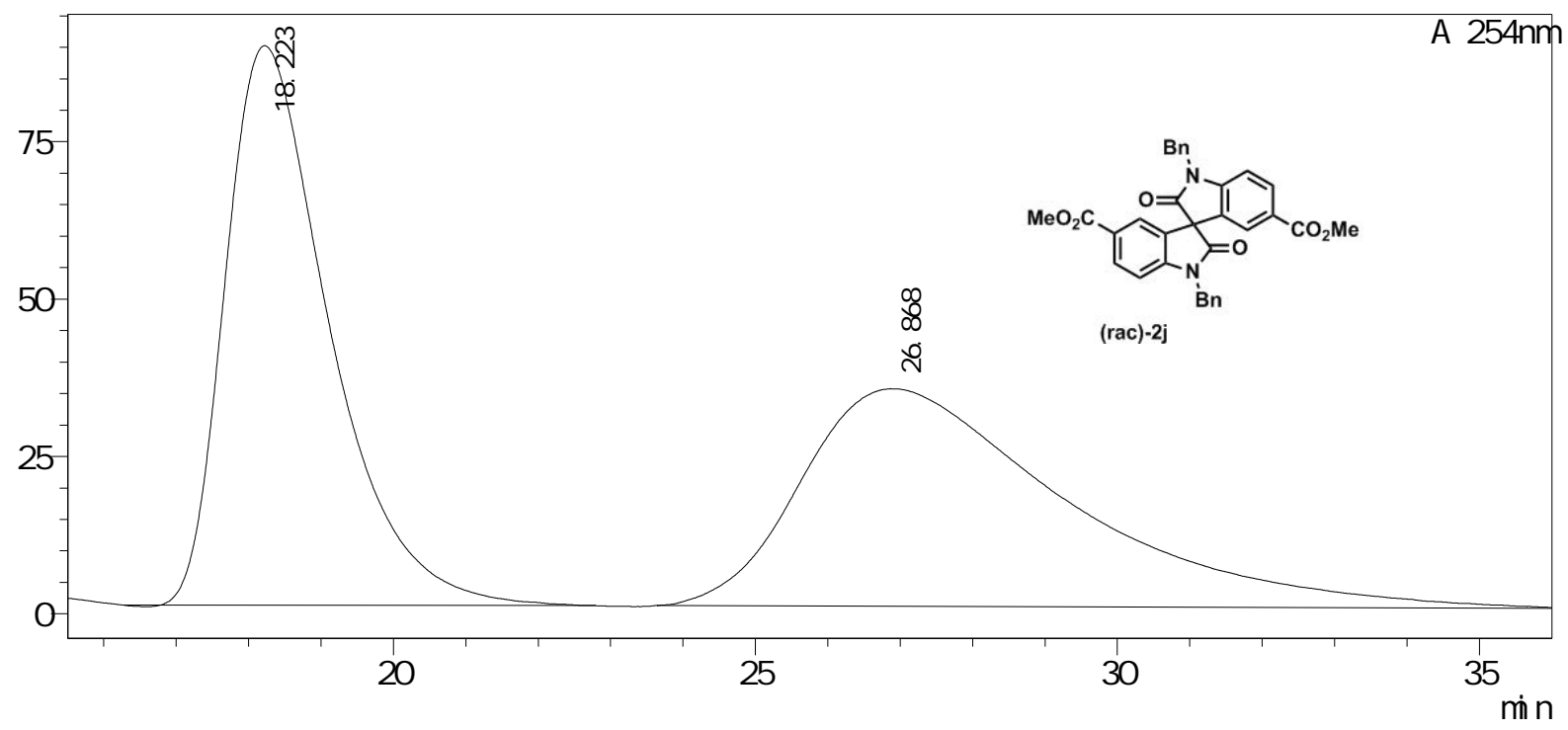

峰表>

检测器 $\mathrm{A} 254 \mathrm{~nm}$

\begin{tabular}{|r|r|r|r|r|r|r|r|}
\hline 峰号 & 保留时间 & \multicolumn{1}{|c|}{ 面积 } & 高度 & 浓度 & 浓度单位 & 标记 & 化合物名 \\
\hline 1 & 18.223 & 8865423 & 88886 & 50.259 & & $\mathrm{M}$ & \\
\hline 2 & 26.868 & 8774216 & 34559 & 49.741 & & $\mathrm{M}$ & \\
\hline 总计 & & 17639638 & 123445 & & & & \\
\hline
\end{tabular}


 \\ LabSolutions 分析报告}

《样品信息>

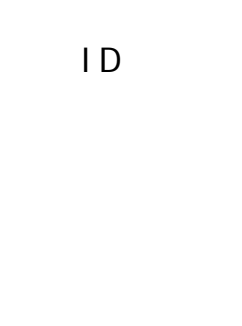

$:$ It

: LTZH JI 1. 21- OD H 40. I cd

: OD H 40\% 100m n. I cm

: $1-1$

$1 \mathrm{uL}$

2019/1/21 10: 56: 26

样品类型：未知

处理日期

2019/1/21 11: 30: 00

$\begin{array}{ll}\text { 分析者 } & \text { Syst em Admi ni st r at or } \\ \text { 处理者 } & \text { : Syst em Admi ni st } r \text { at or }\end{array}$

<色谱图>

nV

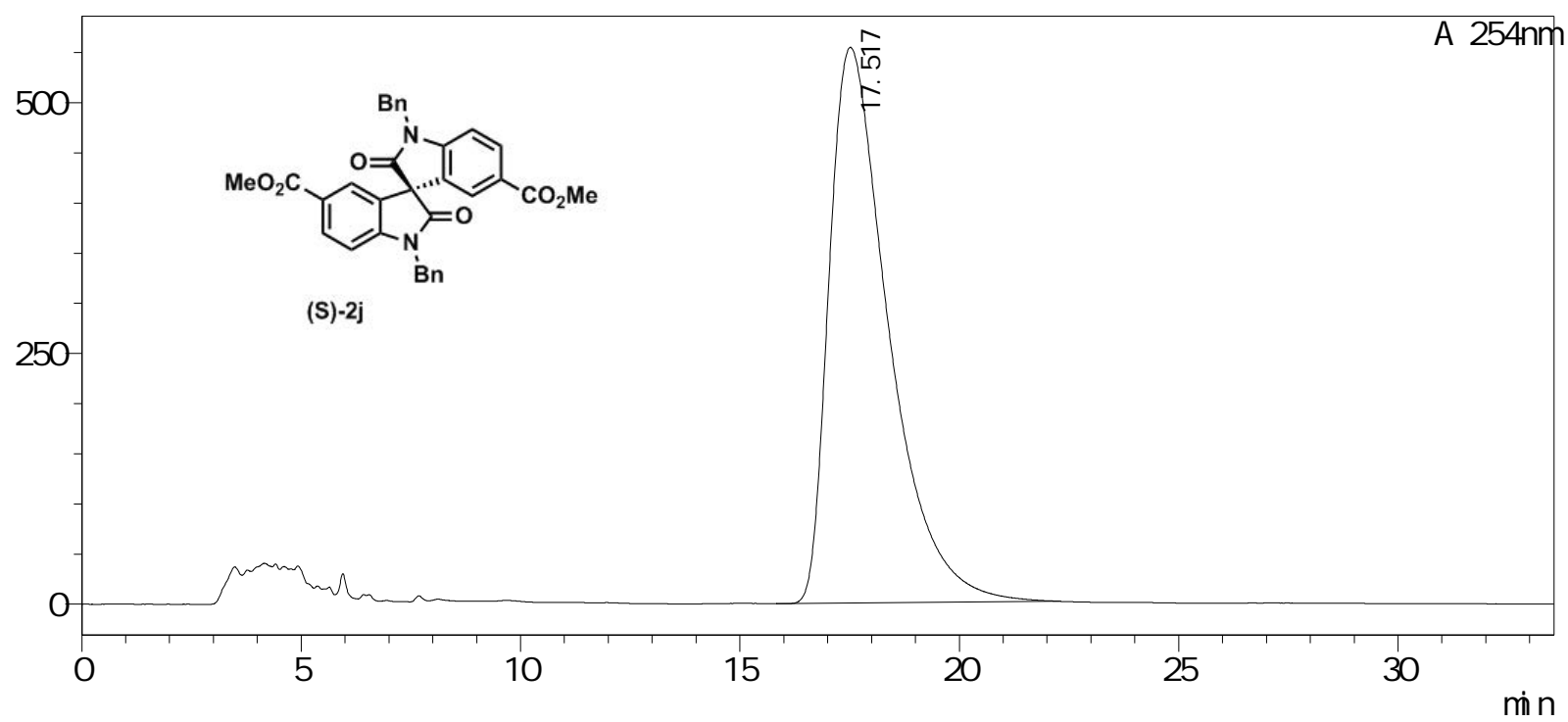

峰表>

检测器 $\mathrm{A} 254 \mathrm{~nm}$

\begin{tabular}{|r|r|r|r|l|l|r|r|}
\hline 峰号 & 保留时间 & 面积 & 高度 & 浓度 & 浓度单位 & 标记 & 化合物名 \\
\hline 1 & 17.517 & 52222119 & 554179 & 100.000 & & $\mathrm{M}$ & \\
\hline 总计 & 52222119 & 554179 & & & & \\
\hline
\end{tabular}


 \\ LabSolutions 分析报告}

《样品信息>

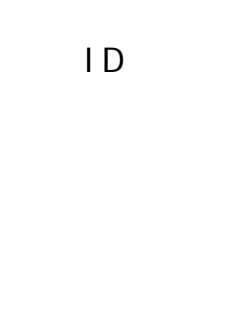

: It

CC20181214- 2. 5- OJ - H 50. I cd

OJ- H 50\% 100mi n. I cm

: $1-1$

$1 \mathrm{uL}$

样品类型 ：未知

处理日期

2019/1/18 15: 20: 17

2019/1/18 17: 07: 37

分析者

- Syst em Adm ni st $r$ at or

处理者 : Syst em Admi ni st $r$ at or

<色谱图>

nV

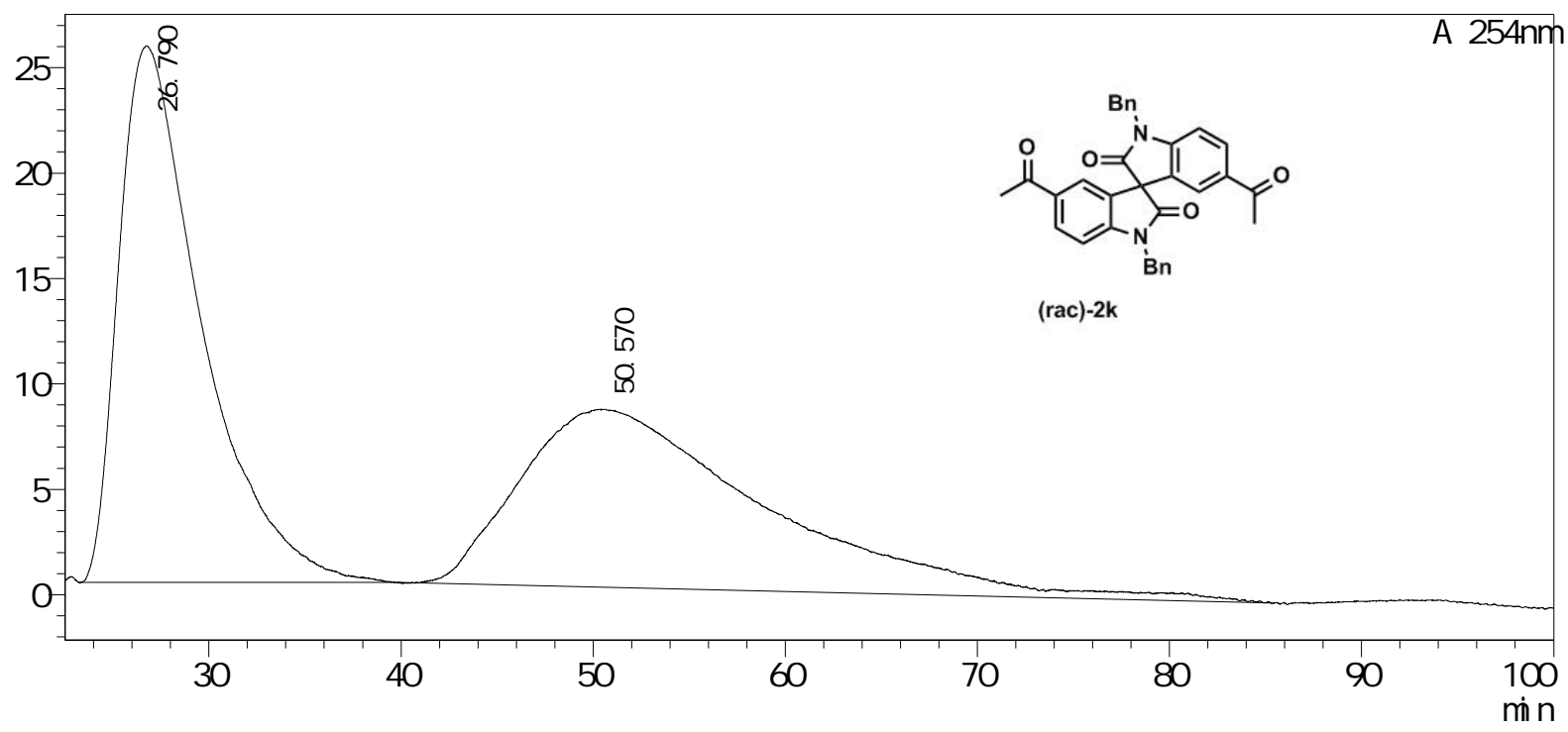

峰表>

检测器A $254 \mathrm{~nm}$

\begin{tabular}{|r|r|r|r|r|r|r|r|}
\hline 峰号 & 保留时间 & \multicolumn{1}{|c|}{ 面积 } & 高度 & \multicolumn{1}{|c|}{ 浓度 } & 浓度单位 & 标记 & 化合物名 \\
\hline 1 & 26.790 & 7612920 & 25453 & 50.402 & & $\mathrm{M}$ & \\
\hline 2 & 50.570 & 7491444 & 8450 & 49.598 & & $\mathrm{M}$ & \\
\hline 总计 & & 15104364 & 33903 & & & & \\
\hline
\end{tabular}


 \\ LabSolutions 分析报告}

《样品信息>

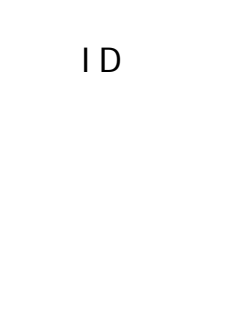

: It

LT201801157. 3 OJ- H 50. I cd

OJ- H 50\% 100mi n. I cm

1- 1

$1 \mathrm{uL}$

2019/1/18 21: 48: 02

样品类型：未知

处理日期

2019/1/18 23: 07: 11

$\begin{array}{ll}\text { 分析者 } & \text { Syst em Admi ni st } r \text { at or } \\ \text { 处理者 } & \text { : Syst em Admi ni st } r \text { at or }\end{array}$

<色谱图>

$\mathrm{nV}$

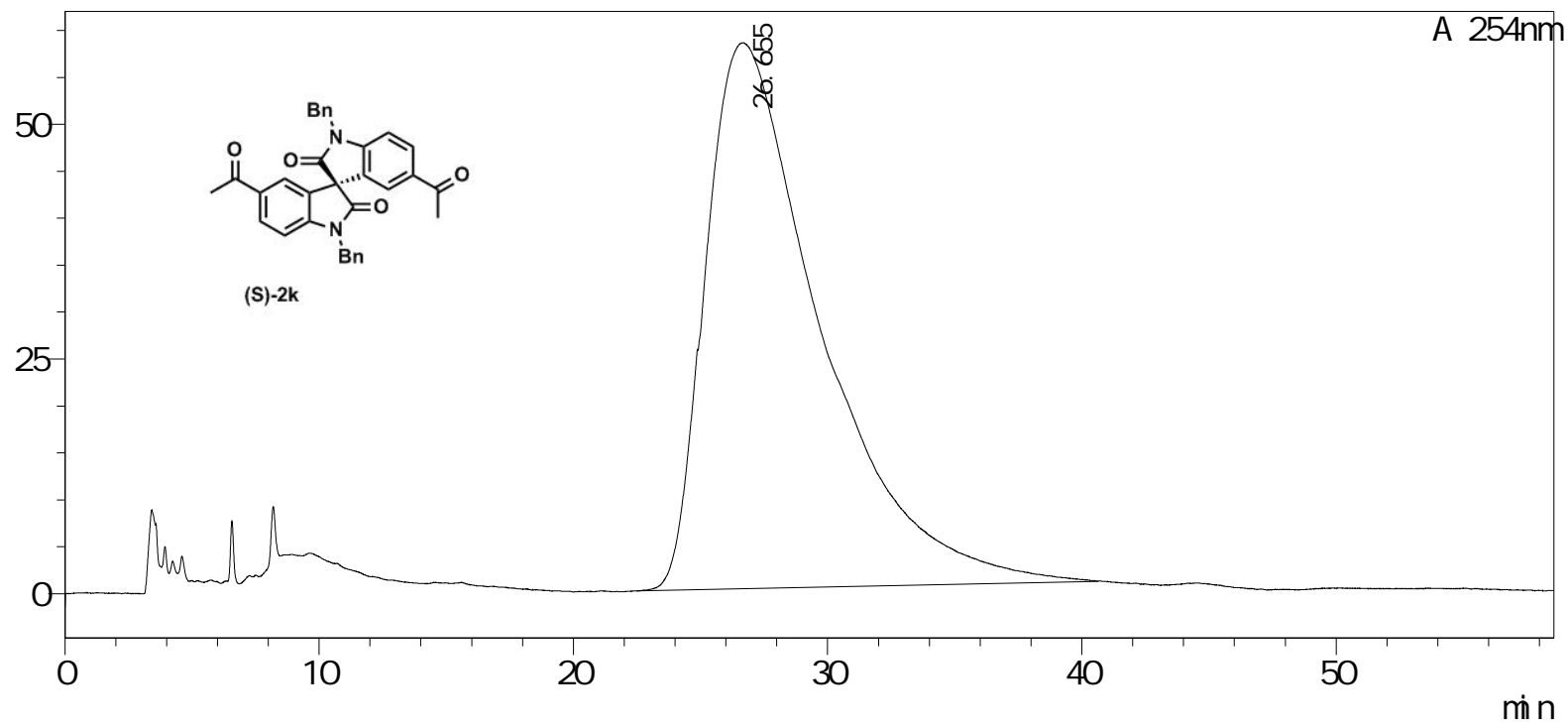

峰表>

检测器A $254 n m$

\begin{tabular}{|r|r|}
\hline 1 & 峰号 \\
\hline 留时间 \\
\hline 总计
\end{tabular}

26. 655

面积

18240679

高度

58145

浓度 100. 000 浓度单位

标记


 \\ LabSolutions 分析报告}

《样品信息>

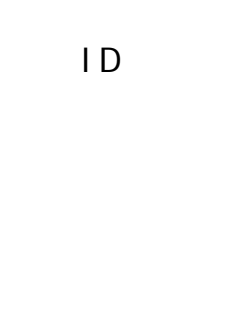

: OYYF

: LT0340 1. 1- AS H 15\% I cd

AS H 30\% 100m n. I cm

1- 1

$1 \mathrm{uL}$

2019/5/10 10: 40: 58

样品类型：未知

处理日期

: 2019/5/10 11: 20: 07

分析者 : Syst em Admi ni st r at or

处理者 : Syst em Admi ni st $r$ at or

<色谱图>

nV

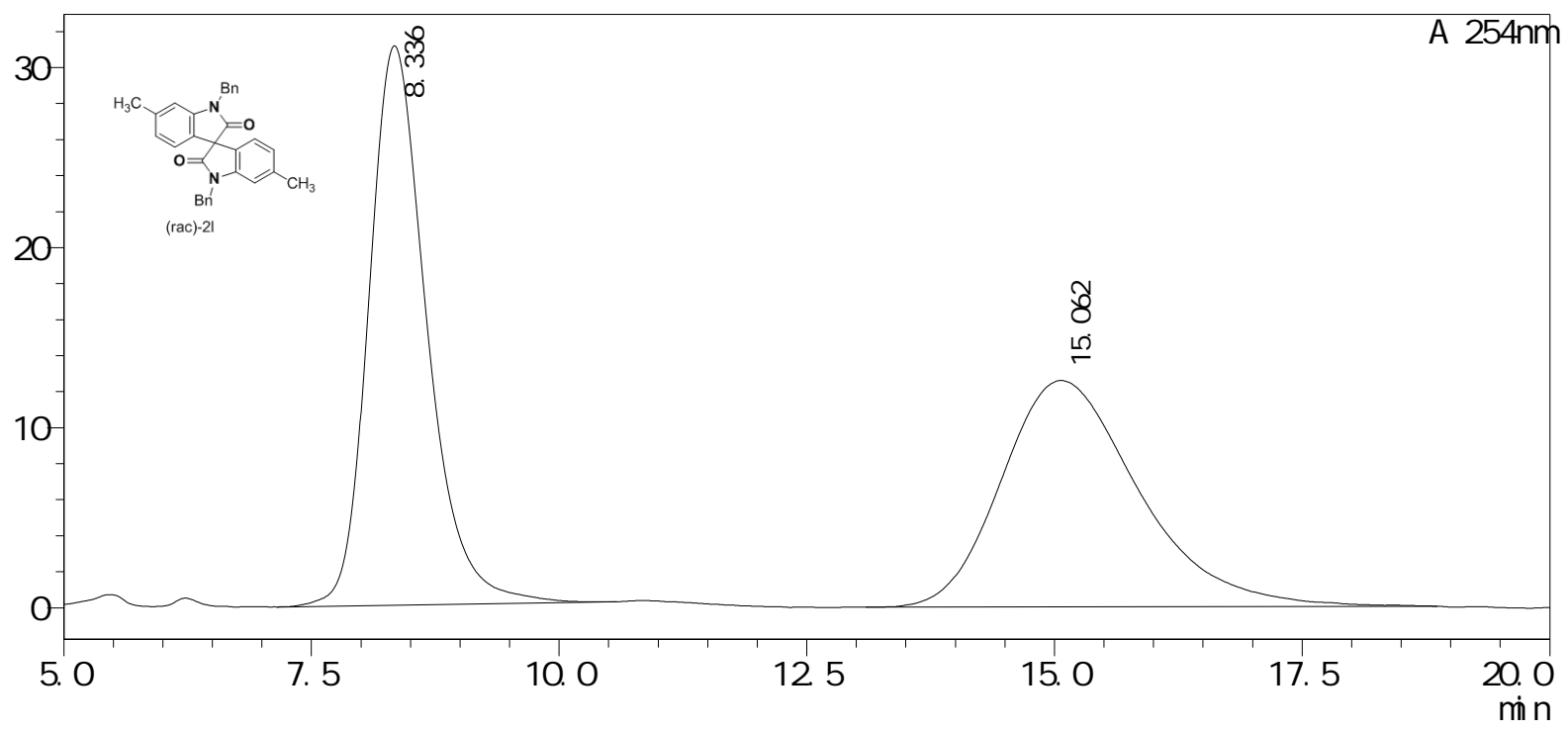

峰表>

检测器A $254 \mathrm{~nm}$

\begin{tabular}{|r|r|r|r|r|r|r|r|}
\hline 峰号 & 保留时间 & 面积 & 高度 & 浓度 & 浓度单位 & 标记 & 化合物名 \\
\hline 1 & 8.336 & 1262808 & 31061 & 50.950 & & $\mathrm{M}$ & \\
\hline 2 & 15.062 & 1215715 & 12573 & 49.050 & & $\mathrm{M}$ & \\
\hline 总计 & & 2478523 & 43635 & & & & \\
\hline
\end{tabular}


 \\ LabSolutions 分析报告}

《样品信息>

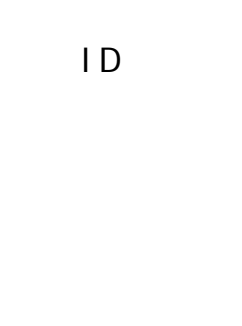

: OYYF

: LT0334 1. 2- AS H 15\% I cd

AS H 30\% 100m n. I cm

1- 1

$1 \mathrm{uL}$

2019/5/10 9: 53: 34

样品类型：未知

2019/5/10 10: 42: 38

分析者 : Syst em Admi ni st r at or

处理者 : Syst em Admi ni st $r$ at or

<色谱图>

nV

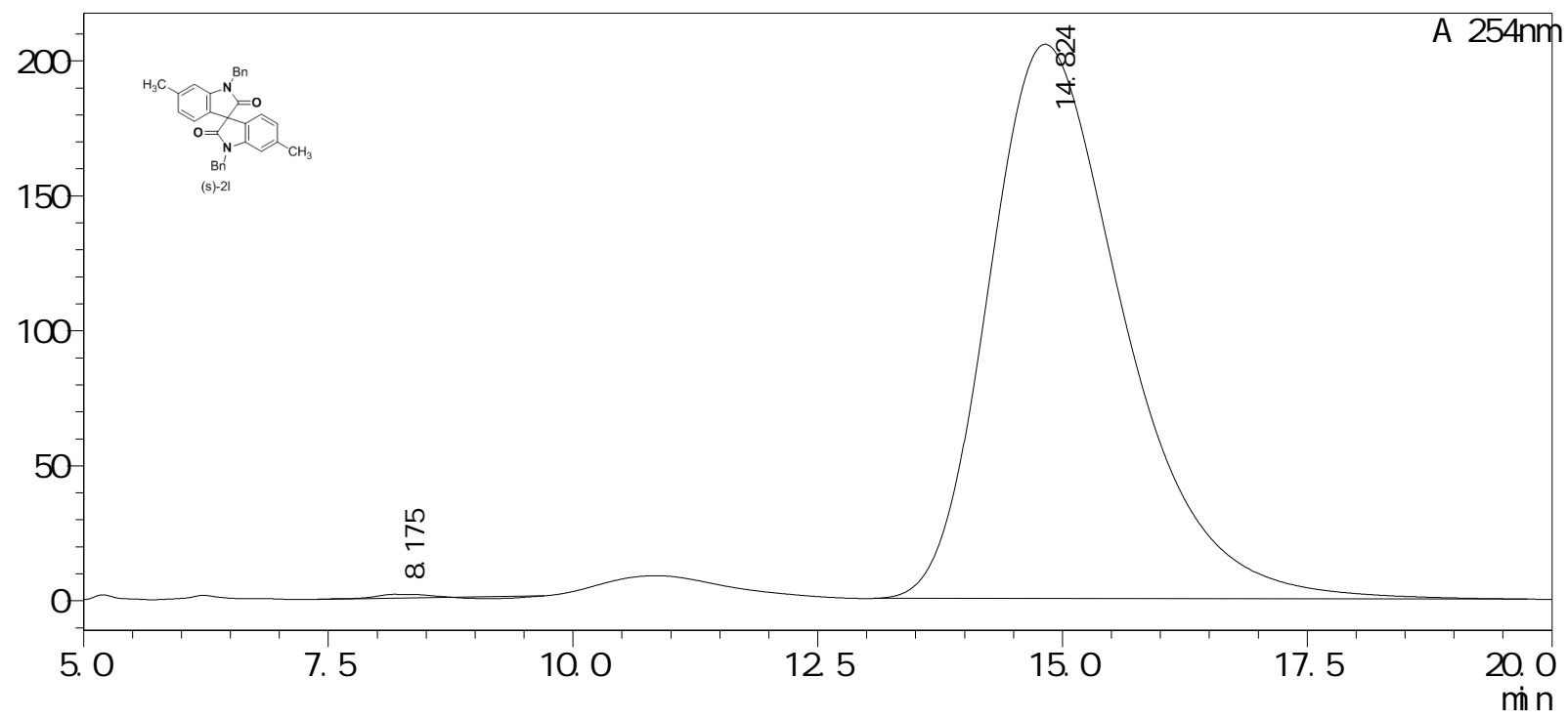

峰表>

检测器 $\mathrm{A} 254 \mathrm{~nm}$

\begin{tabular}{|r|r|r|r|r|r|r|r|}
\hline 峰号 & 保留时间 & \multicolumn{1}{|c|}{ 面积 } & \multicolumn{1}{|c|}{ 高度 } & 浓度 & 浓度单位 & 标记 & 化合物名 \\
\hline 1 & 8.175 & 24257 & 1405 & 0.123 & & $\mathrm{M}$ & \\
\hline 2 & 14.824 & 19745577 & 205346 & 99.877 & & $\mathrm{M}$ & \\
\hline 总计 & & 19769834 & 206751 & & & & \\
\hline
\end{tabular}


 \\ LabSolutions 分析报告}

《样品信息>

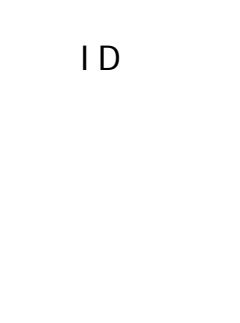

: OYYF- 2941

: LT0339-2-OD H 15\% I cd

: OD H 15\% 40mi n. I cm

: $1-1$

$1 \mathrm{uL}$

2019/5/9 19:52: 50

样品类型 ：未知

2019/5/9 20: 15: 17

分析者 : Syst em Admi ni st r at or

处理者 : Syst em Admi ni st $r$ at or

<色谱图>

nV

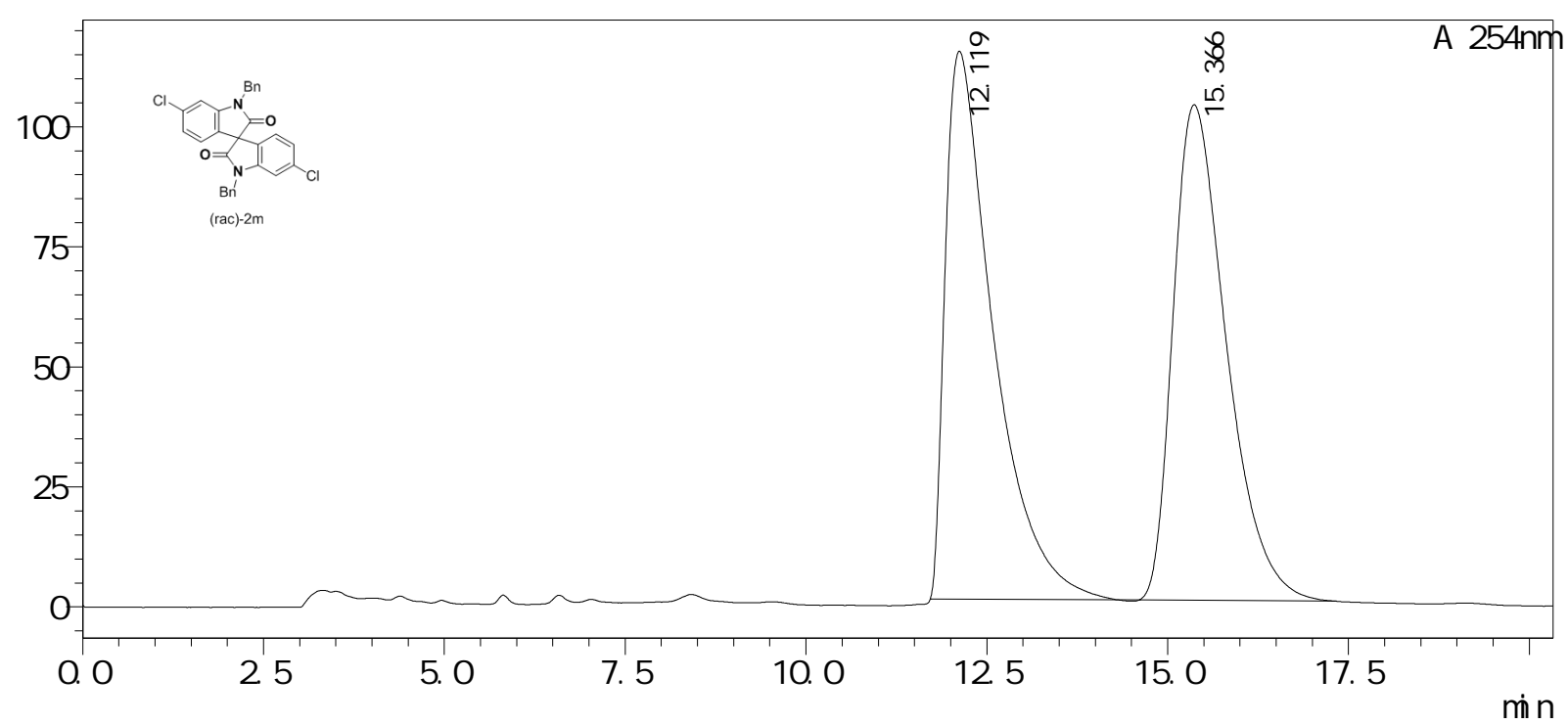

凶峰表>

检测器A $254 \mathrm{~nm}$

峰号 保留时间

\begin{tabular}{|r|r|}
\hline 1 & 12.119 \\
\hline 2 & 15.366 \\
\hline 总计 & \multicolumn{1}{|}{$\mid$}
\end{tabular}

5270225

10548070

\begin{tabular}{|l|}
\hline 高度 \\
\hline 114122 \\
\hline 103213 \\
\hline 217334 \\
\hline
\end{tabular}


 \\ LabSolutions 分析报告}

《样品信息>

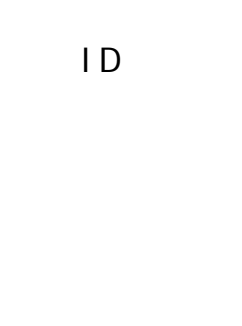

: LT

: LT0339-1CC OD H 15\% I cd

OD H 15\% 40mi n. I cm

1- 1

$1 \mathrm{uL}$

2019/5/12 10: 26: 44

2019/5/12 11: 15: 37

样品类型 ：未知

分析者 : Syst em Admi ni st $r$ at or

处理者 : Syst em Admi ni st $r$ at or

<色谱图>

nV

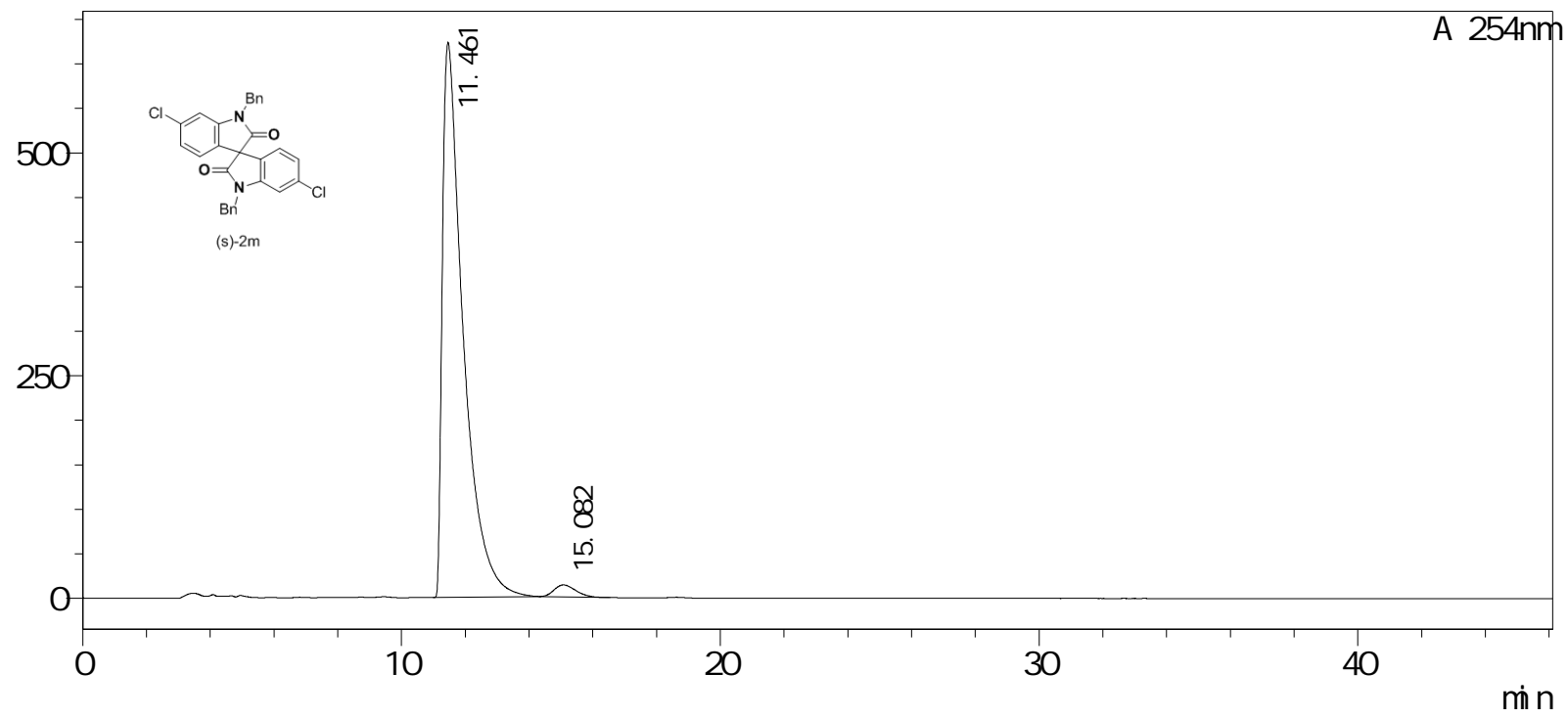

峰表>

检测器A $254 \mathrm{~nm}$

峰号 保留时间

\begin{tabular}{|r|r|}
\hline 1 & 11.461 \\
\hline 2 & 15.082 \\
\hline
\end{tabular}

总计

面积

28256208

高度

630095

28886303

623061
13383

636444

\begin{tabular}{l} 
农度 \\
\hline 97.819 \\
\hline 2.181
\end{tabular}


 \\ LabSolutions 分析报告}

《样品信息>

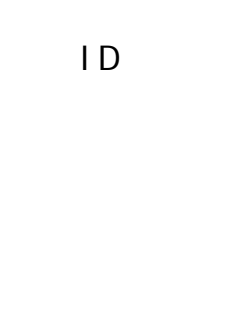

: CC

CC20181201- 1- OD 15. I cd

OD. H $15 \%$ 4Om n. I cm

1- 1

$1 \mathrm{uL}$

2018/12/4 15: 57: 12

样品类型：未知

2018/12/4 16: 29: 21

分析者 : Syst em Admi ni st $r$ at or

处理者 : Syst em Admi ni st $r$ at or

<色谱图>

$\mathrm{nV}$

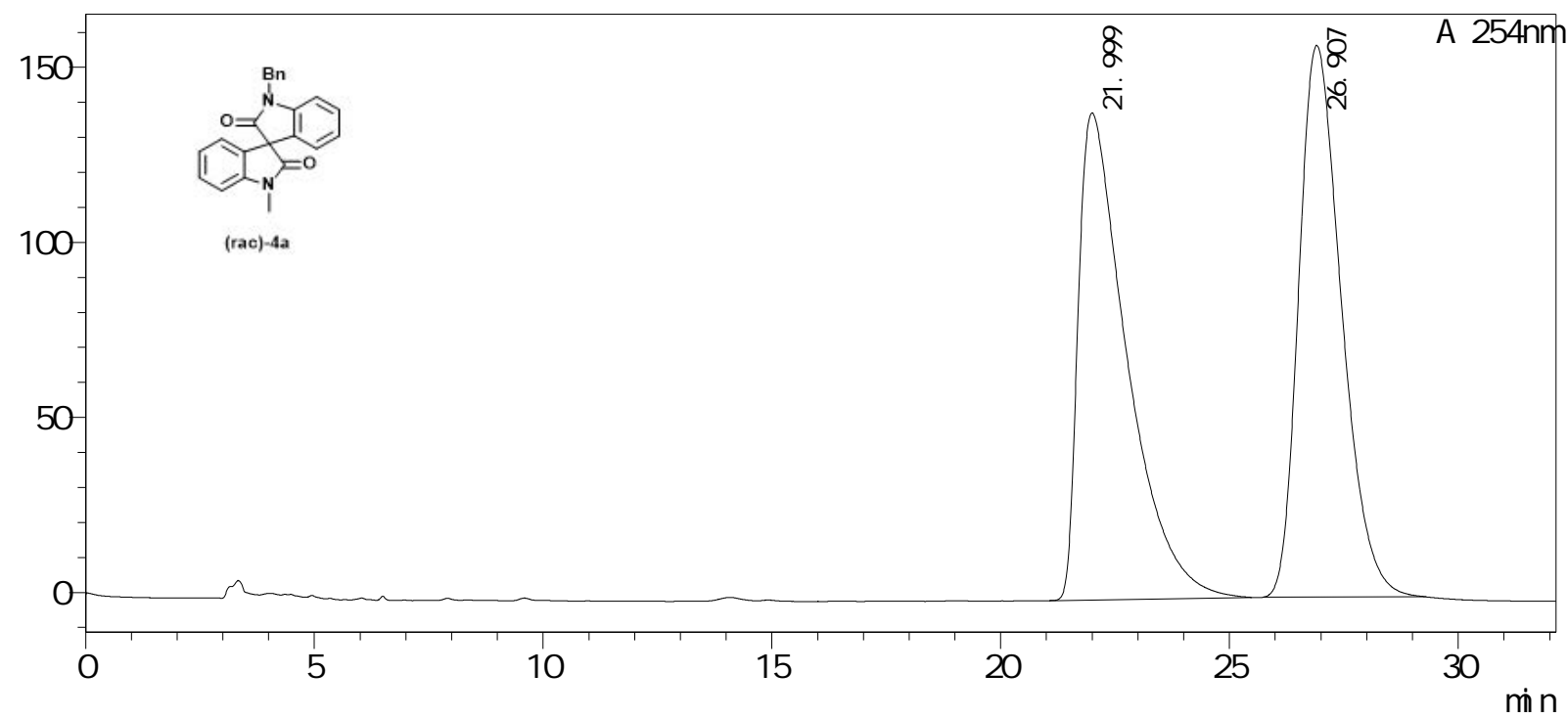

峰表>

检测器A $254 n m$

峰㗊 保留时间

\begin{tabular}{|r|r|}
\hline 1 & 21.999 \\
\hline 2 & 26.907 \\
\hline
\end{tabular}

面积

10257624

20472811

\begin{tabular}{|l|}
\hline 高度 \\
\hline 139133 \\
\hline 157606 \\
\hline 296739 \\
\hline
\end{tabular}

浓度 浓度单位

标记

50. 104

49.896

$\mathrm{M}$

总计 
 \\ LabSolutions 分析报告}

《样品信息>

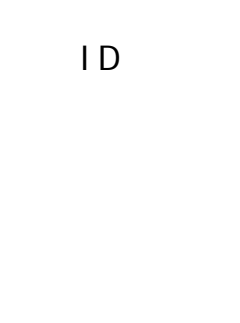

: CC

LT201801130 OD 15\% I cd

OD H 15\% 40m n. I cm

1- 1

$1 \mathrm{uL}$

2018/12/6 9: 06: 11

2018/12/6 9: 46: 26
样品类型 ：未知

分析者 : Syst em Admi ni st r at or

处理者 $\quad$ Syst em Admi ni st $r$ at or

<色谱图>

nV

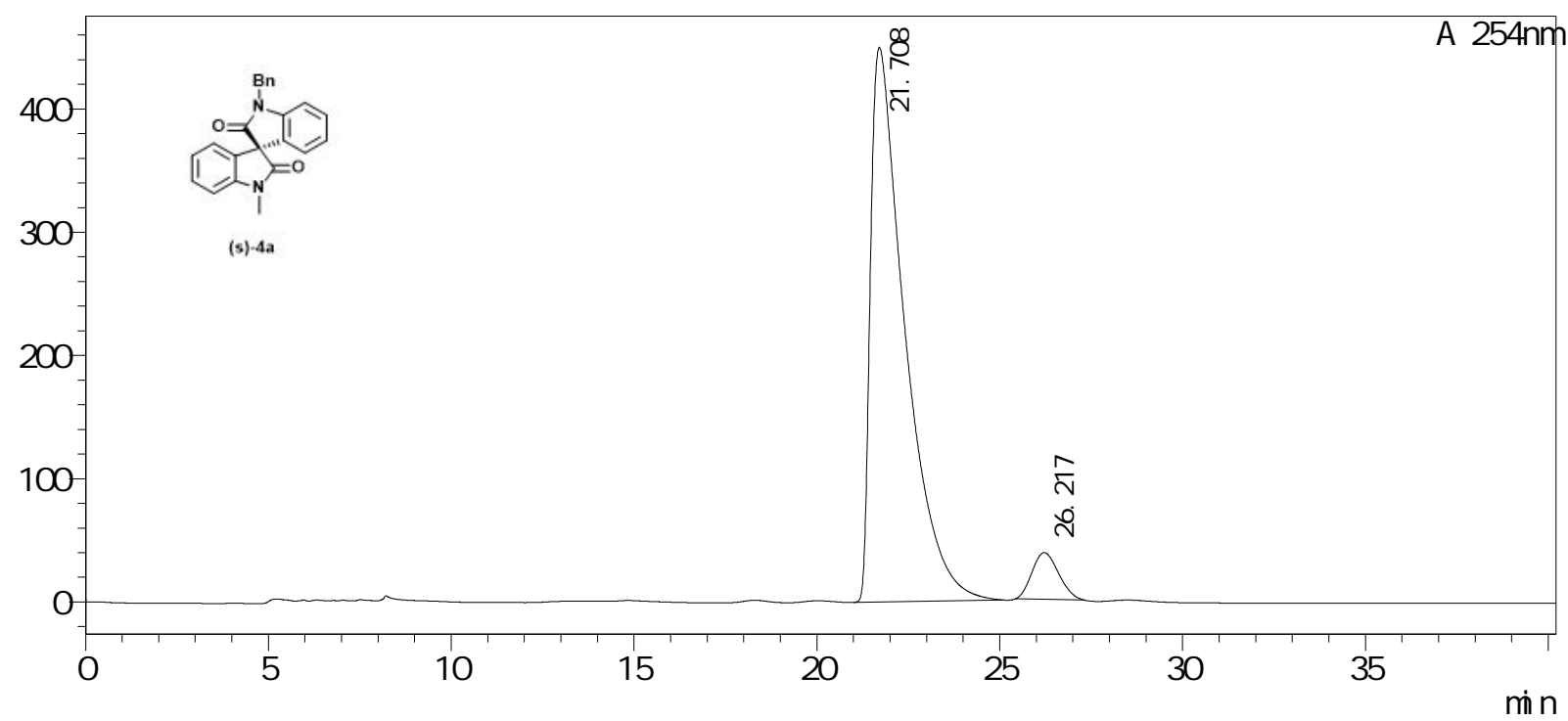

峰表>

检测器 $\mathrm{A} 254 \mathrm{~nm}$

\begin{tabular}{|r|r|r|r|r|r|r|r|}
\hline 峰号 & 保留时间 & \multicolumn{1}{|c|}{ 面积 } & \multicolumn{1}{|c|}{ 高度 } & \multicolumn{1}{|c|}{ 浓度 } & 浓度单位 & 标记 & 化合物名 \\
\hline 1 & 21.708 & 29313215 & 450063 & 93.887 & & $\mathrm{M}$ & \\
\hline 2 & 26.217 & 1908534 & 37802 & 6.113 & & $\mathrm{M}$ & \\
\hline 总计 & & 31221750 & 487865 & & & & \\
\hline
\end{tabular}


 \\ LabSolutions 分析报告}

《样品信息>

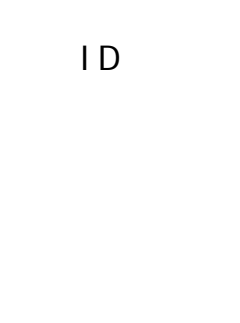

: CC

CC20181201- 2- OD 5\% I cd

OD. H $5 \% 35 \mathrm{~min}$. I cm

: $1-1$

$1 \mathrm{uL}$

2018/12/5 10: 49: 01

样品类型：未知

2018/12/5 14: 14: 47

分析者 : Syst em Admi ni st r at or

处理者 : Syst em Admi ni st $r$ at or

<色谱图>

$\mathrm{nV}$

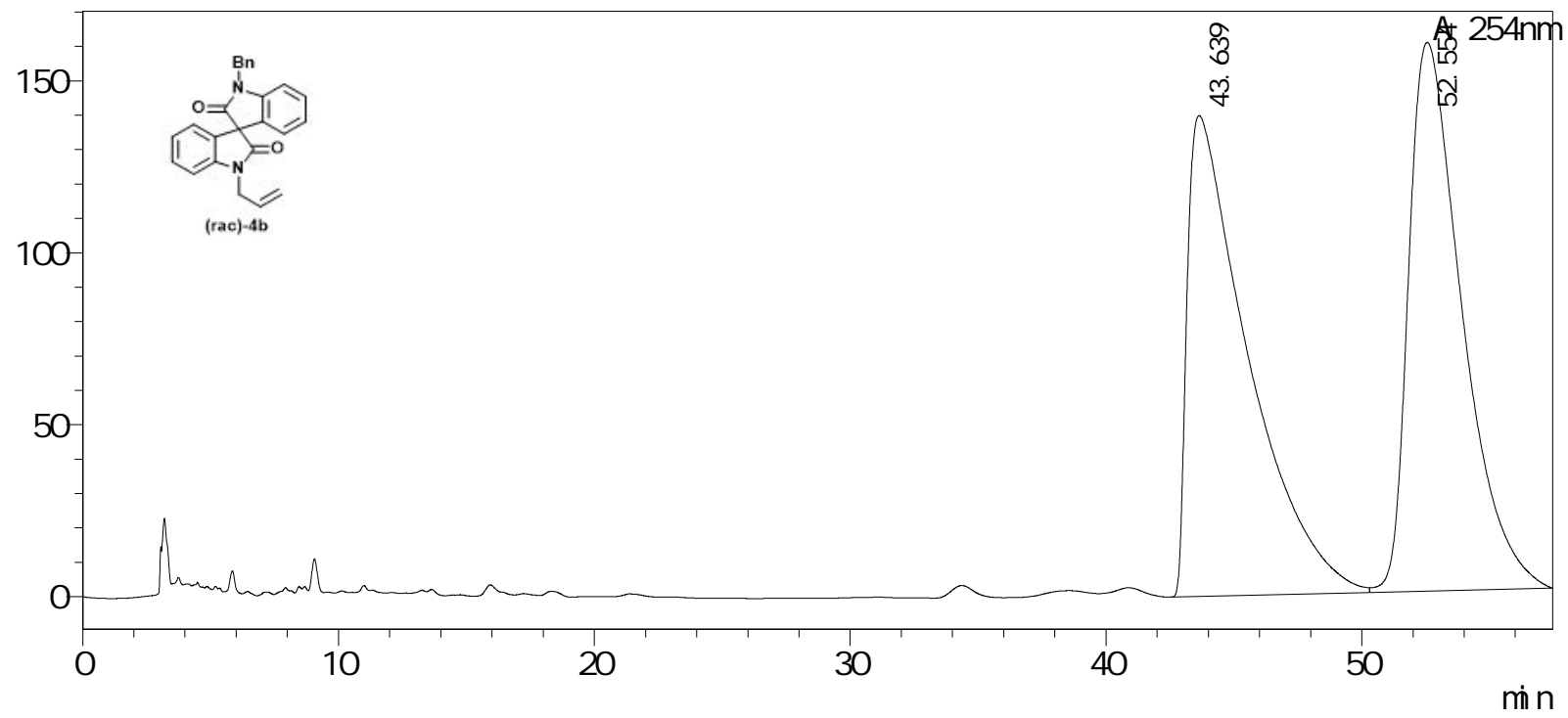

《峰表>

检测器A $254 \mathrm{~nm}$

峰咢保卯时间

\begin{tabular}{|r|r|r|r|}
\hline 峰号 & 保留时间 & 面积 & 高度 \\
\hline 1 & 43.639 & 23131112 & 139895 \\
\hline 2 & 52.554 & 23103349 & 159596 \\
\hline 总计 & & 46234461 & 299490 \\
\hline
\end{tabular}


 \\ LabSolutions 分析报告}

《样品信息>

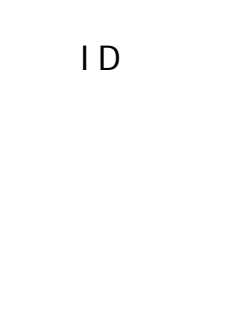

: CC

LT201801131- OD 5\% I cd

: OD H $5 \% 35 \mathrm{mi} \mathrm{n} . \mathrm{cm}$

1- 1

$1 \mathrm{uL}$

2018/12/6 9: 56: 09

样品类型：未知

2018/12/6 11: 30: 26

分析者

: Syst em Admi ni st $r$ at or

处理者 : Syst em Admi ni st $r$ at or

<色谱图>

nV

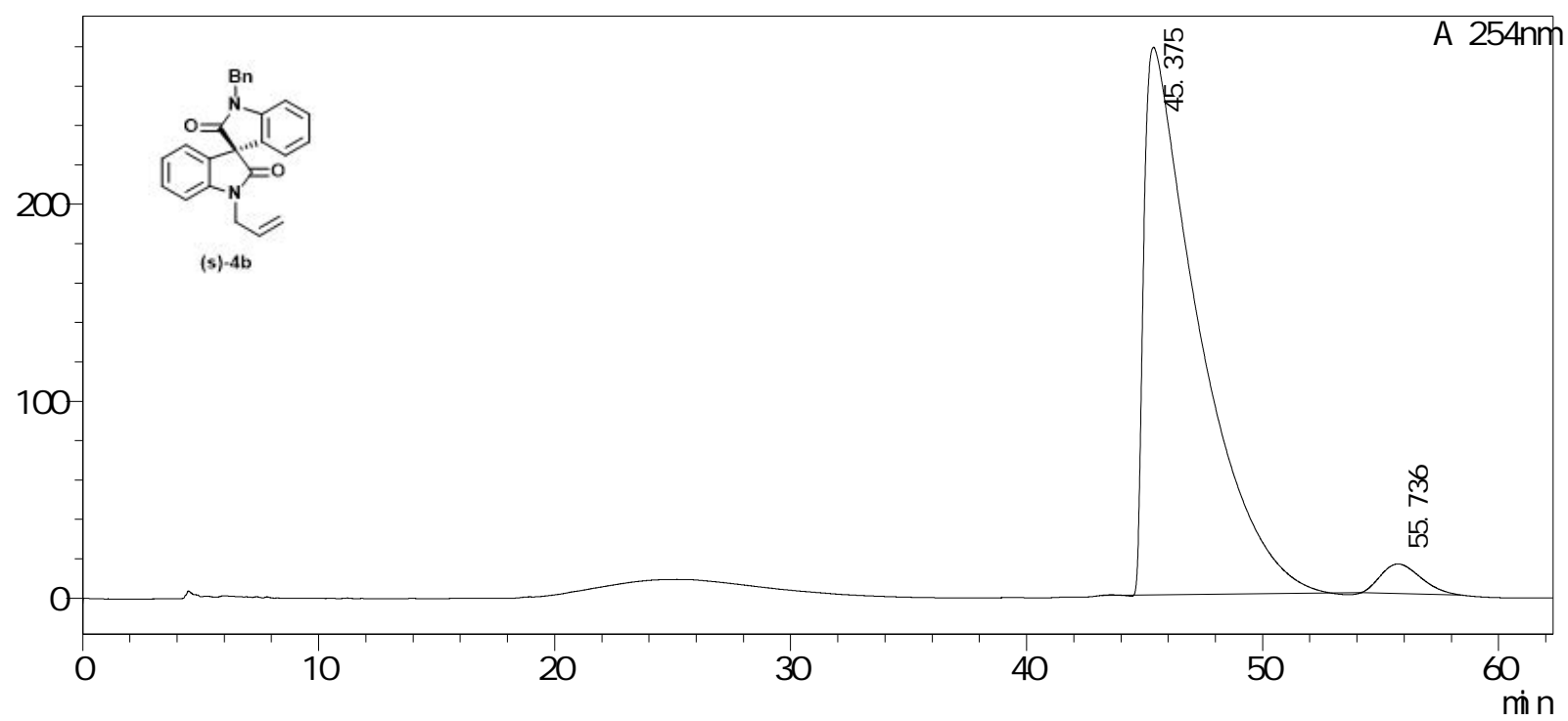

峰表>

检测器 $\mathrm{A} 254 \mathrm{~nm}$

\begin{tabular}{|r|r|r|r|r|r|r|r|}
\hline \multicolumn{1}{|c|}{ 峰号 } & 保留时间 & \multicolumn{1}{|c|}{ 面积 } & \multicolumn{1}{|c|}{ 高 } & \multicolumn{1}{|c|}{ 浓度 } & 浓度单位 & 标记 & 化合物名 \\
\hline 1 & 45.375 & 44825307 & 278049 & 96.201 & & $\mathrm{M}$ & \\
\hline 2 & 55.736 & 1770272 & 14998 & 3.799 & & $\mathrm{M}$ & \\
\hline 总计 & & 46595580 & 293047 & & & & \\
\hline
\end{tabular}


 \\ LabSolutions 分析报告}

《样品信息>

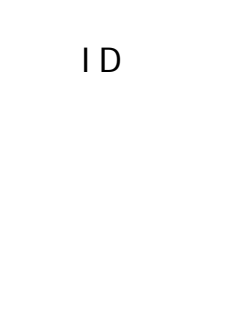

$:$ It

CC20181208- 1. 2- OD 10. I cd

OD. $\mathrm{H} .10 \% 30 \mathrm{~min} . \mathrm{lcm}$

1- 1

$1 \mathrm{uL}$

2018/12/17 22: 42: 53

样品类型 ：未知

: 2018/12/17 23: 22: 27

分析者

- Syst em Admi ni st $r$ at or

处理者 : Syst em Admi ni st $r$ at or

<色谱图>

nV

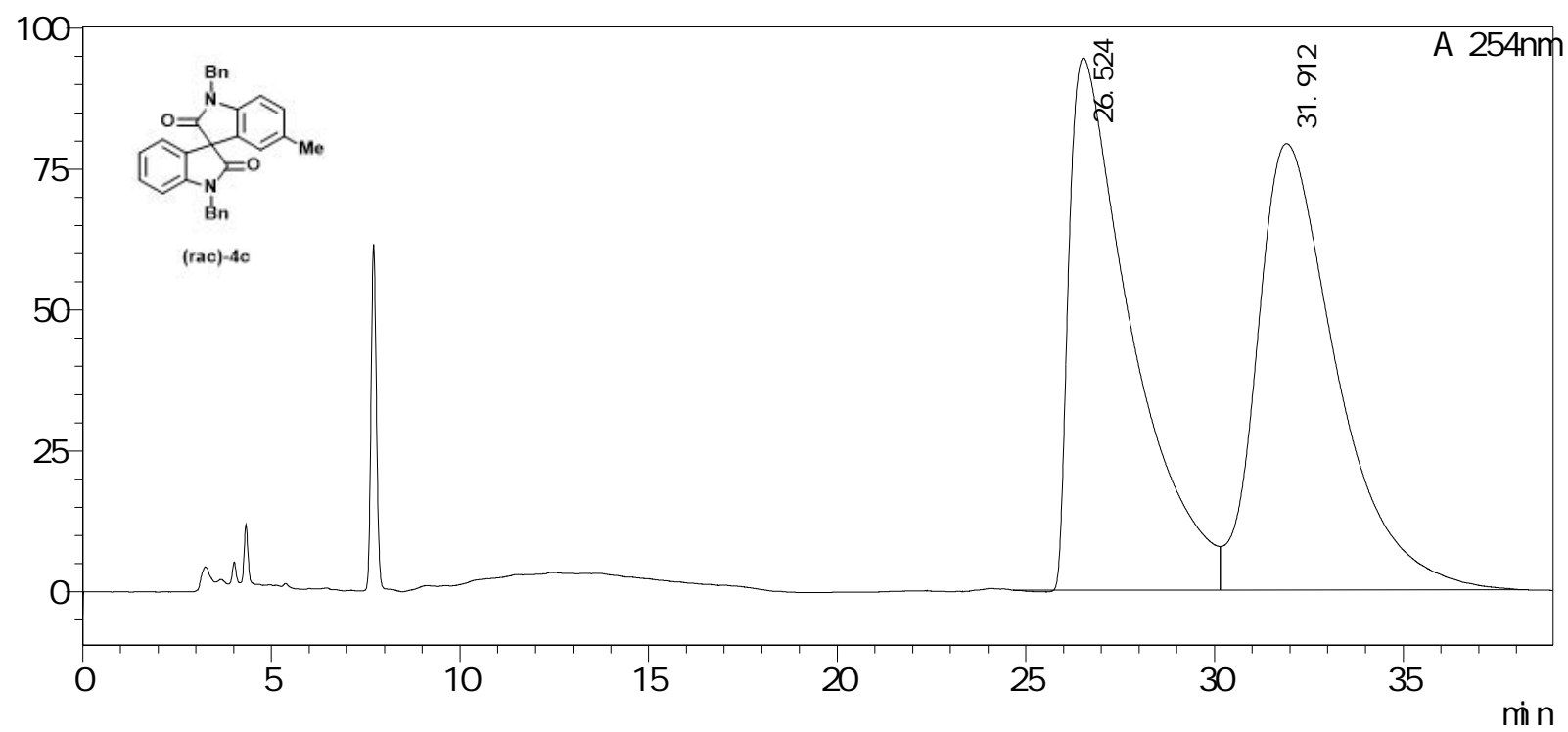

《峰表>

检测器A $254 \mathrm{~nm}$

峰咢保留时间

\begin{tabular}{|r|r|}
\hline 1 & 26.524 \\
\hline 2 & 31.912 \\
\hline
\end{tabular}

面积 10827010 高度 11606328 22433338

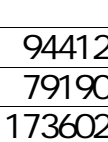

\begin{tabular}{r|r} 
标记 & 化合物名 \\
\hline$M$ & \\
\hline $\mathrm{VM}$ &
\end{tabular}


 \\ LabSolutions 分析报告}

《样品信息>

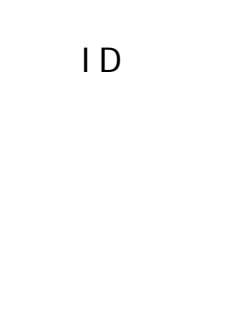

: It

LT201801140. 2- OD H 10. I cd

OD. H $10 \% 30 \mathrm{~min}$. I cm

1- 1

$1 \mathrm{uL}$

2019/1/17 22: 29: 58

样品类型：未知

处理日期

2019/1/18 11: 29: 07

分析者 : Syst em Admi ni st r at or

处理者 : Syst em Admi ni st $r$ at or

<色谱图>

nV

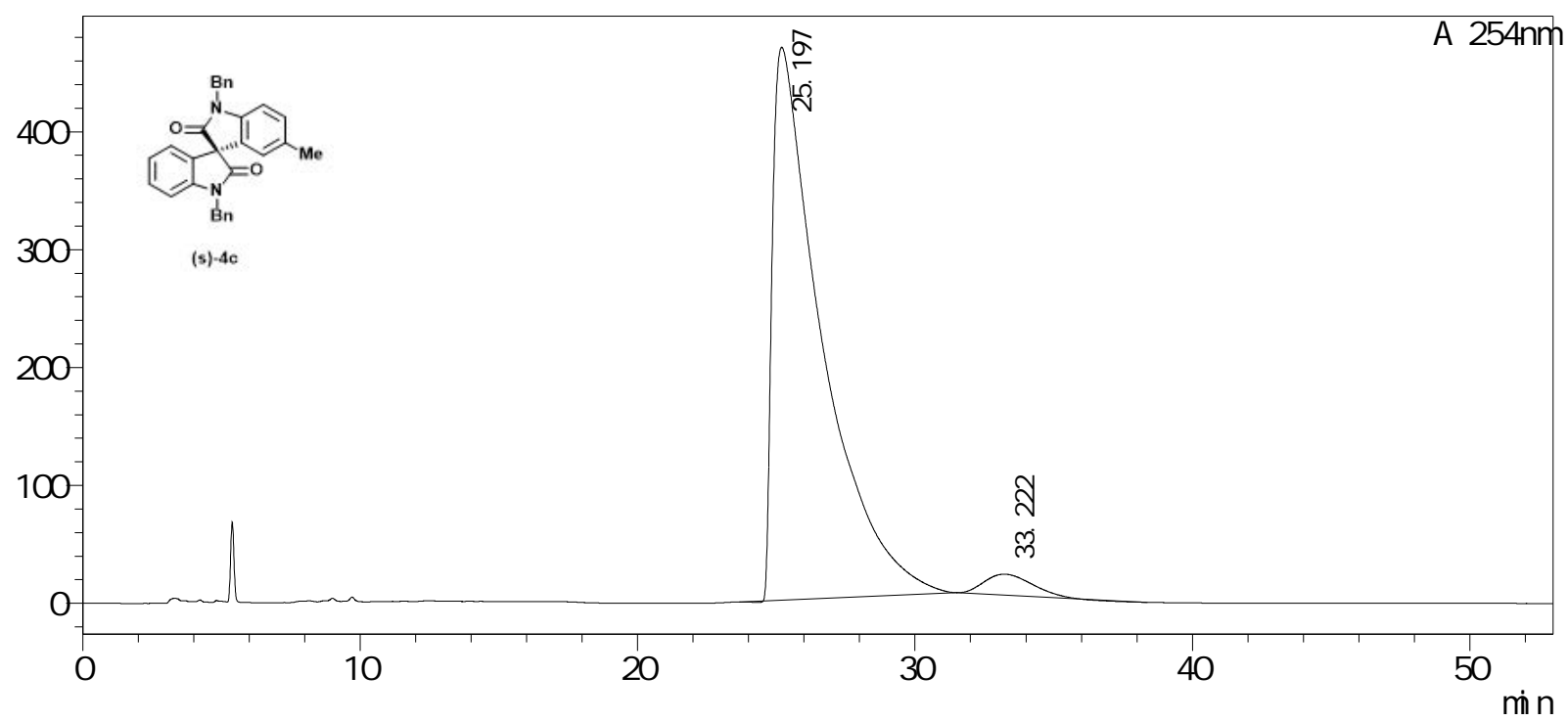

凶峰表>

检测器A $254 \mathrm{~nm}$

\begin{tabular}{|r|r|r|r|r|r|r|r|}
\hline 峰号 & 保留时间 & \multicolumn{1}{|c|}{ 面积 } & \multicolumn{1}{|c|}{ 高度 } & \multicolumn{1}{|c|}{ 浓度 } & 浓度单位 & 标记 & 化合物名 \\
\hline 1 & 25.197 & 58023645 & 468945 & 96.473 & & $\mathrm{M}$ & \\
\hline 2 & 33.222 & 2121551 & 17839 & 3.527 & & $\mathrm{M}$ & \\
\hline 总计 & & 60145196 & 486783 & & & & \\
\hline
\end{tabular}


 \\ LabSolutions 分析报告}

《样品信息>

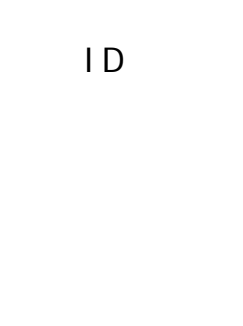

$:$ It

: LT201802072- OJ- H 50. I cd

OJ- H. 50\% 100mi n. I cm

: $1-1$

$1 \mathrm{uL}$

: 2019/1/22 20: 13: 17

: 2019/1/22 21: 59: 57

样品类型：未知

分析者 : Syst em Admi ni st r at or

处理者 : Syst em Admi ni st $r$ at or

<色谱图>

nV

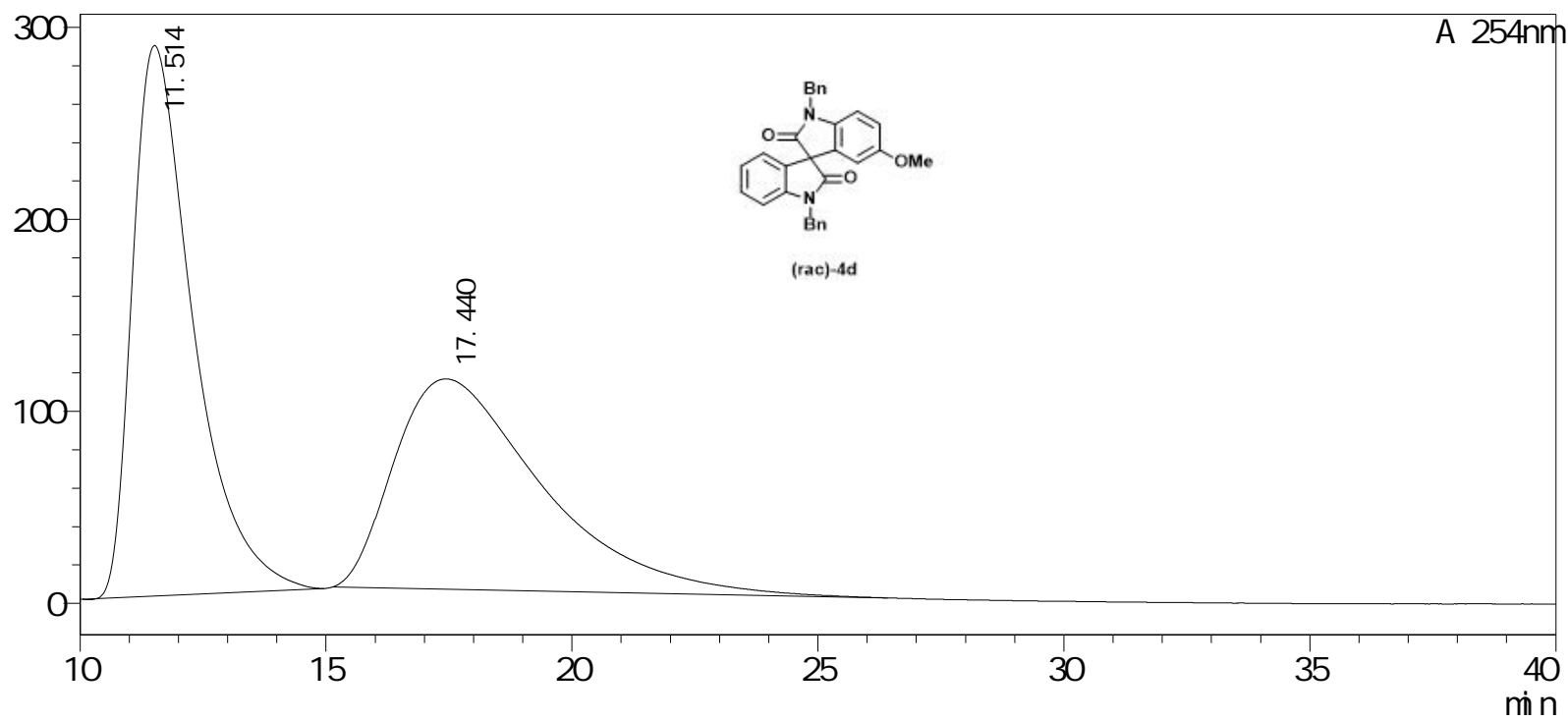

《峰表>

检测器A $254 \mathrm{~nm}$

\begin{tabular}{|r|r|r|r|r|r|r|r|}
\hline 峰号 & 保留时间 & \multicolumn{1}{|c|}{ 面积 } & 高度 & 浓度 & 浓度单位 & 标记 & 化合物名 \\
\hline 1 & 11.514 & 24626890 & 286760 & 51.737 & & $\mathrm{M}$ & \\
\hline 2 & 17.440 & 22973325 & 109554 & 48.263 & & $\mathrm{M}$ & \\
\hline 总计 & & 47600215 & 396314 & & & & \\
\hline
\end{tabular}


 \\ LabSolutions 分析报告}

《样品信息>

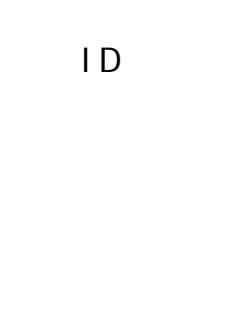

: It

LT201802071. 1- OJ- H 50. I cd

OJ- H 50\% 100mi n. I cm

1- 1

$1 \mathrm{uL}$

2019/1/23 21: 12: 08

样品类型：未知

处理日期

2019/1/24 20: 50: 23

分析者

- System Admini st rat or

处理者 : Syst em Admi ni st $r$ at or

<色谱图>

$\mathrm{mV}$

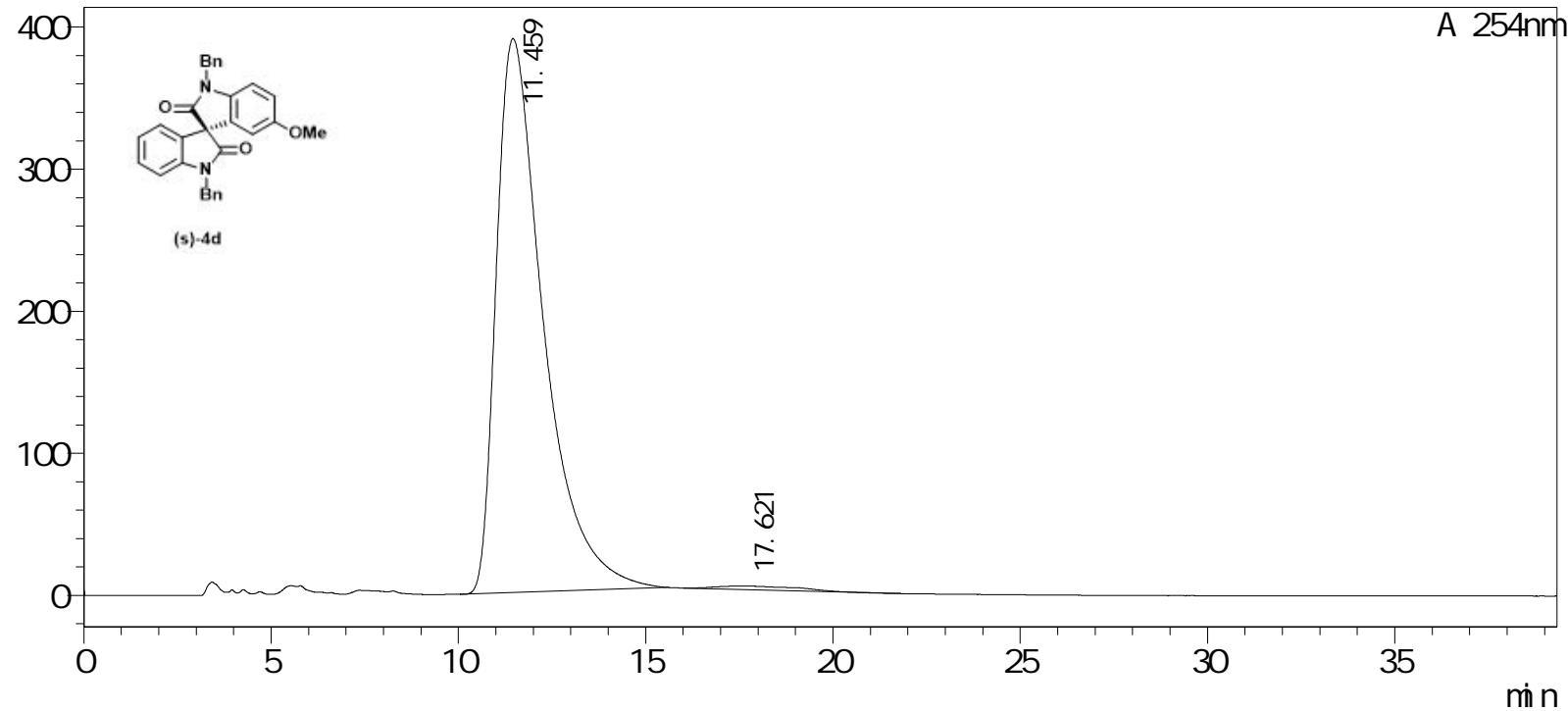

《峰表>

检测器A $254 \mathrm{~nm}$

峰号 保留时间

\begin{tabular}{r|r|}
\hline 1 & 11.459 \\
\hline 2 & 17.621 \\
\hline
\end{tabular}

总计

面积

34236736

高度

浓度

98.792

34655517

392625

1. 208

浓度单位

\begin{tabular}{r|r} 
标记 & 化合物名 \\
\hline$M$ & \\
$M$ & \\
\hline
\end{tabular}


 \\ LabSolutions 分析报告}

《样品信息>

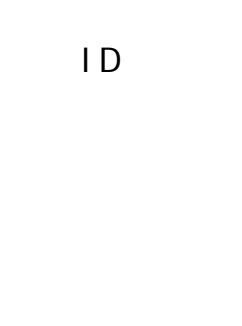

$:$ It

CC20181218 1- AS H 20. I cd

AS H $20 \% 100 \mathrm{~m} \mathrm{n}$. I cm

1- 1

$1 \mathrm{uL}$

2018/12/20 16: 26: 27

样品类型：未知

处理日期

: 2018/12/20 22: 40: 45

分析者 : Syst em Admi ni st $r$ at or

处理者 : Syst em Admi ni st $r$ at or

<色谱图>

nV

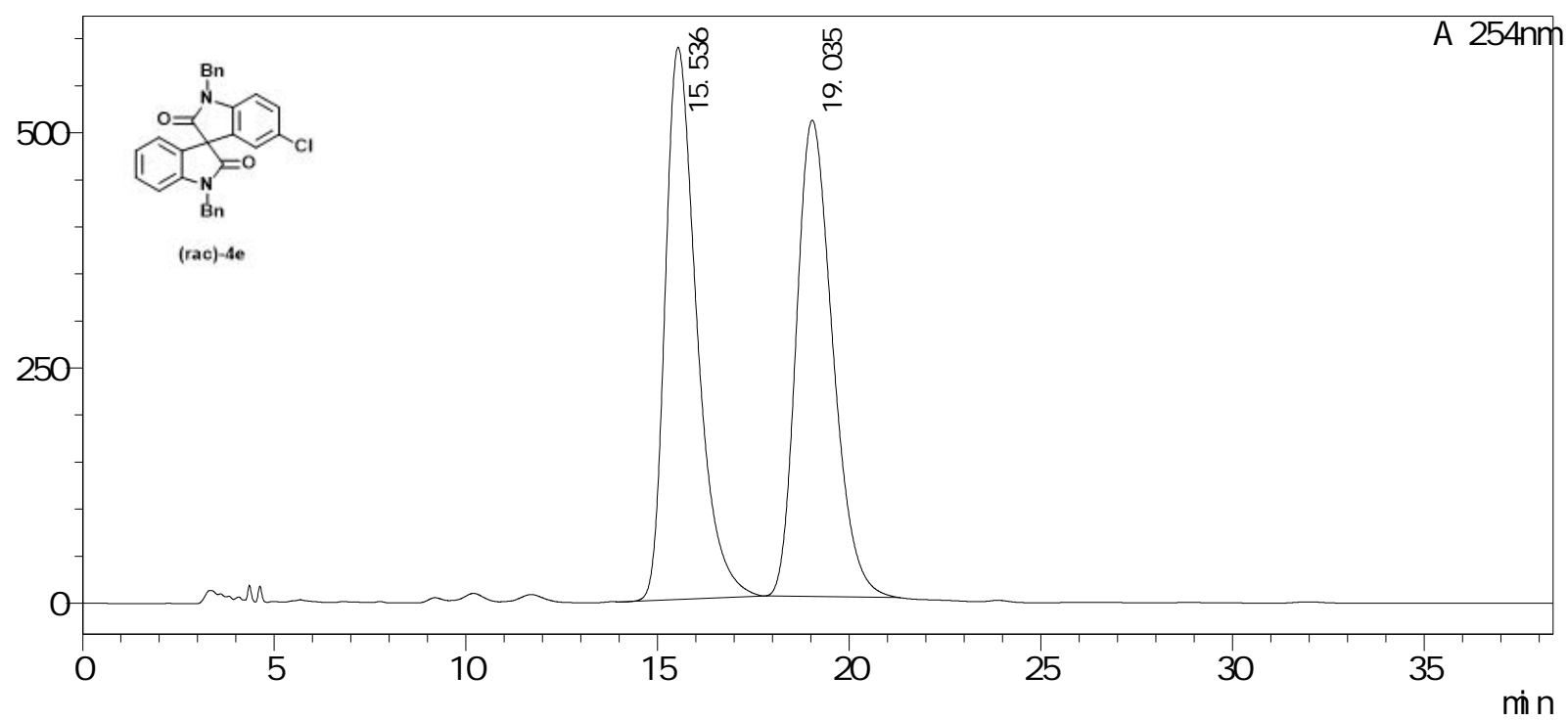

峰表>

检测器A $254 \mathrm{~nm}$

峰咢保留时间

\begin{tabular}{|r|r|}
\hline 1 & 15.536 \\
\hline 2 & 19.035 \\
\hline 总计 & \\
\hline
\end{tabular}

面积

33495265

586890

浓度

50.188

49.812

浓度单位

\begin{tabular}{|r|r|}
\hline 标记 & 化合物名 \\
\hline$M$ & \\
\hline$M$ & \\
\hline
\end{tabular}


 \\ LabSolutions 分析报告}

《样品信息>

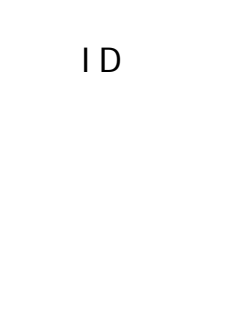

$:$ It

: LT201802007- AS H 20. I cd

AS H $20 \% 100 \mathrm{mi} \mathrm{n.} \mathrm{I} \mathrm{cm}$

1- 1

$1 \mathrm{uL}$

2018/12/20 17:06: 25

样品类型：未知

处理日期

: 2018/12/21 11: 44: 26

分析者

- Syst em Admi ni st r at or

处理者 $\quad$ Syst em Admi ni st $r$ at or

<色谱图>

$\mathrm{nV}$

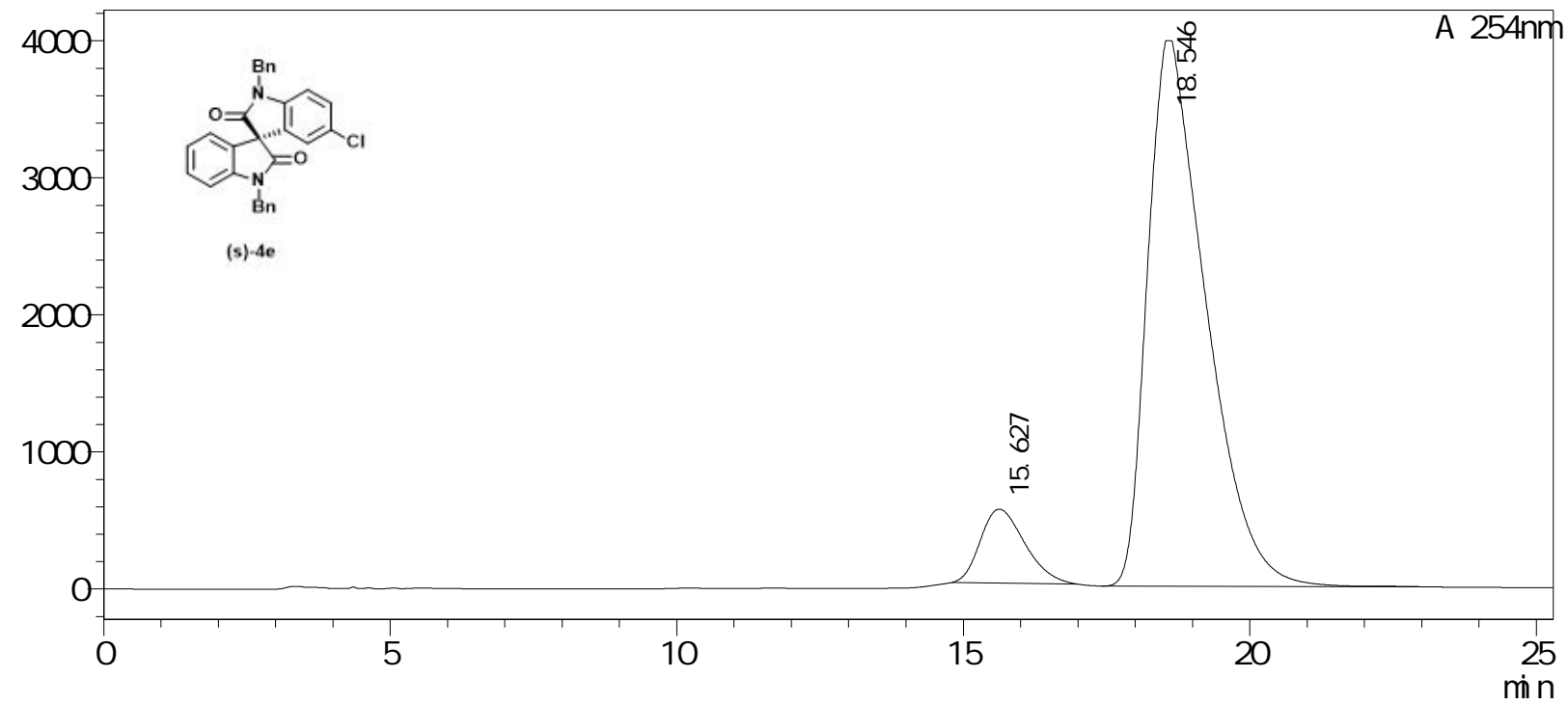

峰表>

检测器A $254 \mathrm{~nm}$

\begin{tabular}{|r|r|r|r|r|r|r|r|}
\hline \multicolumn{1}{|c|}{ 峰号 } & 保留时间 & \multicolumn{1}{|c|}{ 面积 } & \multicolumn{1}{|c|}{ 高度 } & \multicolumn{1}{|c|}{ 浓度 } & 浓度单位 & 标记 & 化合物名 \\
\hline 1 & 15.627 & 29027808 & 538609 & 9.331 & & $\mathrm{M}$ & \\
\hline 2 & 18.546 & 282046711 & 3979465 & 90.669 & & $\mathrm{M}$ & \\
\hline 总计 & & 311074519 & 4518073 & & & & \\
\hline
\end{tabular}


 \\ LabSolutions 分析报告}

<样品信息>

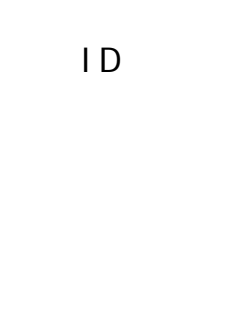

: It

CC20181208- 2. 2- AS H 20. I cd

AS $\mathrm{H} 20 \%$ 100m n. I cm

1- 1

$1 \mathrm{uL}$

样品类型 ：未知

处理日期

2019/1/18 9: 52: 30

2019/1/18 11: 31: 12

分析者 : Syst em Admi ni st r at or

处理者 : Syst em Admi ni st $r$ at or

<色谱图>

$\mathrm{nV}$

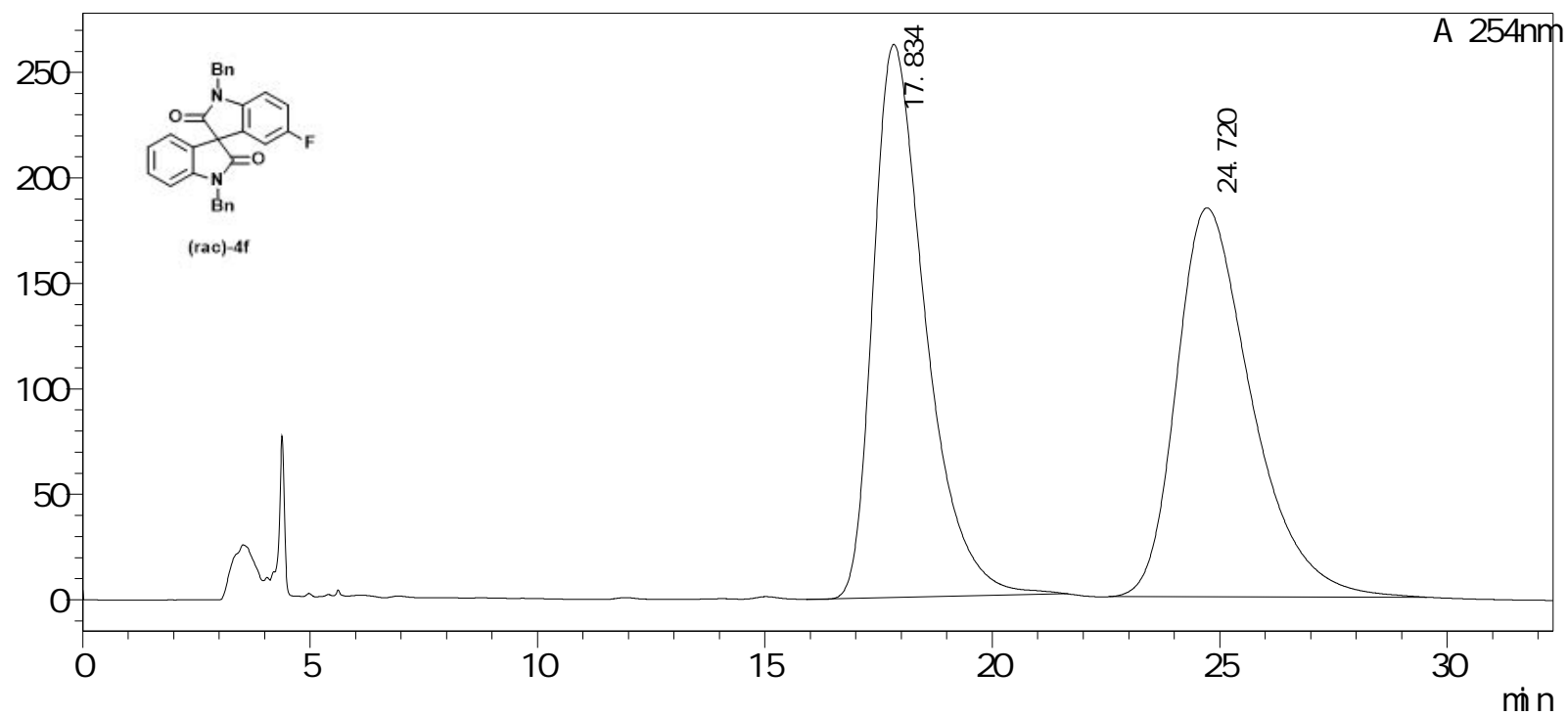

峰表>

检测器A $254 \mathrm{~nm}$

峰咢保留时间

\begin{tabular}{|r|r|}
\hline 1 & 17.834 \\
\hline 2 & 24.720 \\
\hline 总计 & \\
\hline
\end{tabular}

面积

21197918

42223220

高度
262291
184399
446690

浓度

49. 796 浓度单位

标记


 \\ LabSolutions 分析报告}

《样品信息>

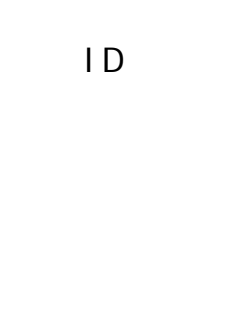

: It

: LT201802039. 2-AS H 20. I cd AS H $20 \% 100 \mathrm{mi}$. I cm

1- 1

$1 \mathrm{uL}$

样品类型：未知

处理日期

2019/1/18 10: 27: 47

2019/1/18 11: 31: 18

分析者

处理者

: Syst em Admi ni st $r$ at or

: Syst em Admi ni st $r$ at or

<色谱图>

nV

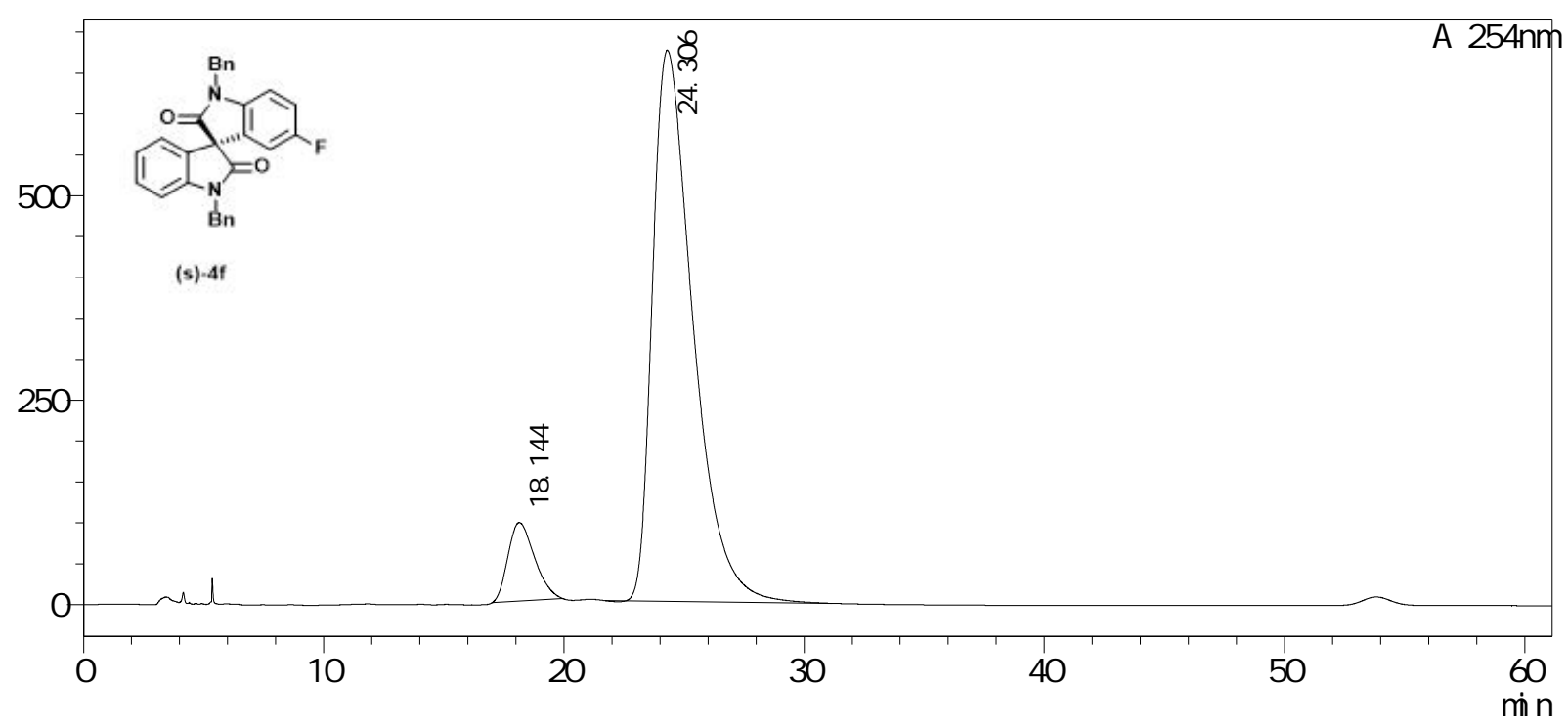

《峰表>

检测器A $254 \mathrm{~nm}$

检财器A

\begin{tabular}{|r|r|r|r|r|r|r|r|}
\hline 峰号 & 保留时间 & \multicolumn{1}{|c|}{ 面积 } & \multicolumn{1}{|c|}{ 高度 } & \multicolumn{1}{|c|}{ 浓度 } & 浓度单位 & 标记 & 化合物名 \\
\hline 1 & 18.144 & 7358058 & 95880 & 8.520 & & $\mathrm{M}$ & \\
\hline 2 & 24.306 & 79006016 & 674239 & 91.480 & & $\mathrm{M}$ & \\
\hline 总计 & & 86364074 & 770118 & & & & \\
\hline
\end{tabular}


 \\ LabSolutions 分析报告}

《样品信息>

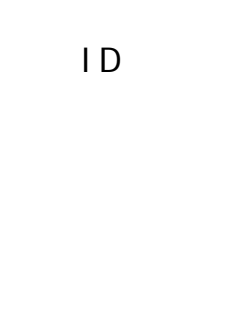

: It

CC20181226- 1- OD 30+TFA. I cd

OD. H $30 \% 60 \mathrm{mi} \mathrm{n}$. I cm

: $1-1$

$1 \mathrm{uL}$

样品类型 ：未知

处理日期

2018/12/28 14: 58: 55

2018/12/28 16: 12: 55

分析者

- System Adm ni st r at or

处理者 : Syst em Admi ni st $r$ at or

<色谱图>

$\mathrm{nV}$

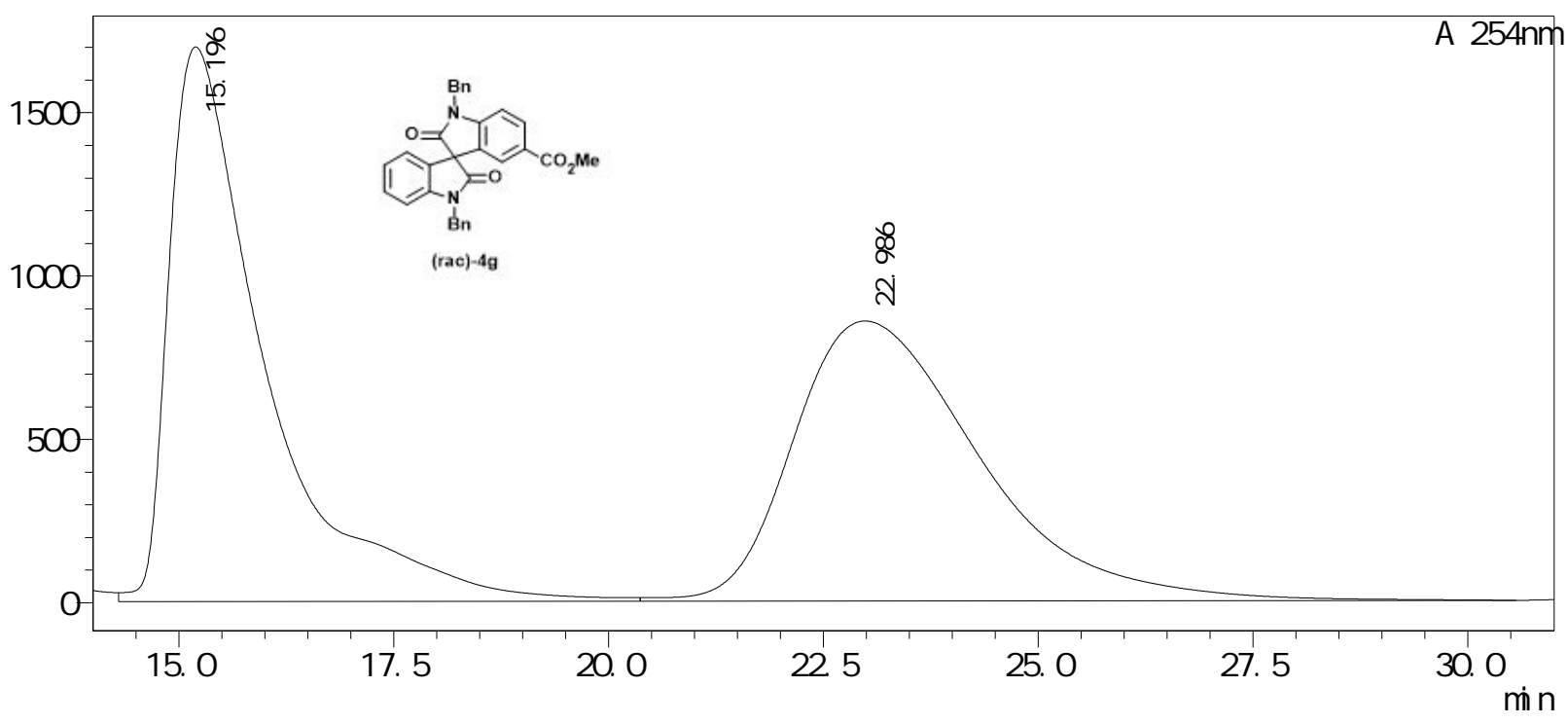

凶峰表>

检测器A $254 \mathrm{~nm}$

峰号 保留时间

\begin{tabular}{|r|r|}
\hline 1 & 15.196 \\
\hline 2 & 22.986 \\
\hline
\end{tabular}

面积

高度

132112273

261610950

$\begin{array}{r}\multicolumn{1}{|l}{\text { 高度 }} \\ \hline 1697813 \\ \hline 856956 \\ \hline 2554769\end{array}$

浓度

50.500

49.500

浓度单位

标记

化合物名

总计

2554769 
 \\ LabSolutions 分析报告}

《样品信息>

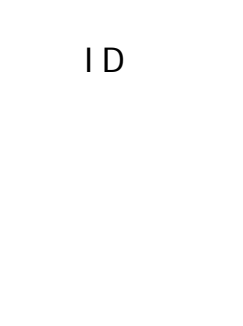

: It

LT201802027. 1- OD H 30. I cd

OD. H $30 \% 60 \mathrm{mi} \mathrm{n}$. I cm

1- 1

$1 \mathrm{uL}$

样品类型：未知

处理日期

2019/1/17 16: 50: 21

分析者

: Syst em Admi ni st $r$ at or

2019/1/17 18: 51: 56

处理者

: Syst em Admi ni st $r$ at or

<色谱图>

nV

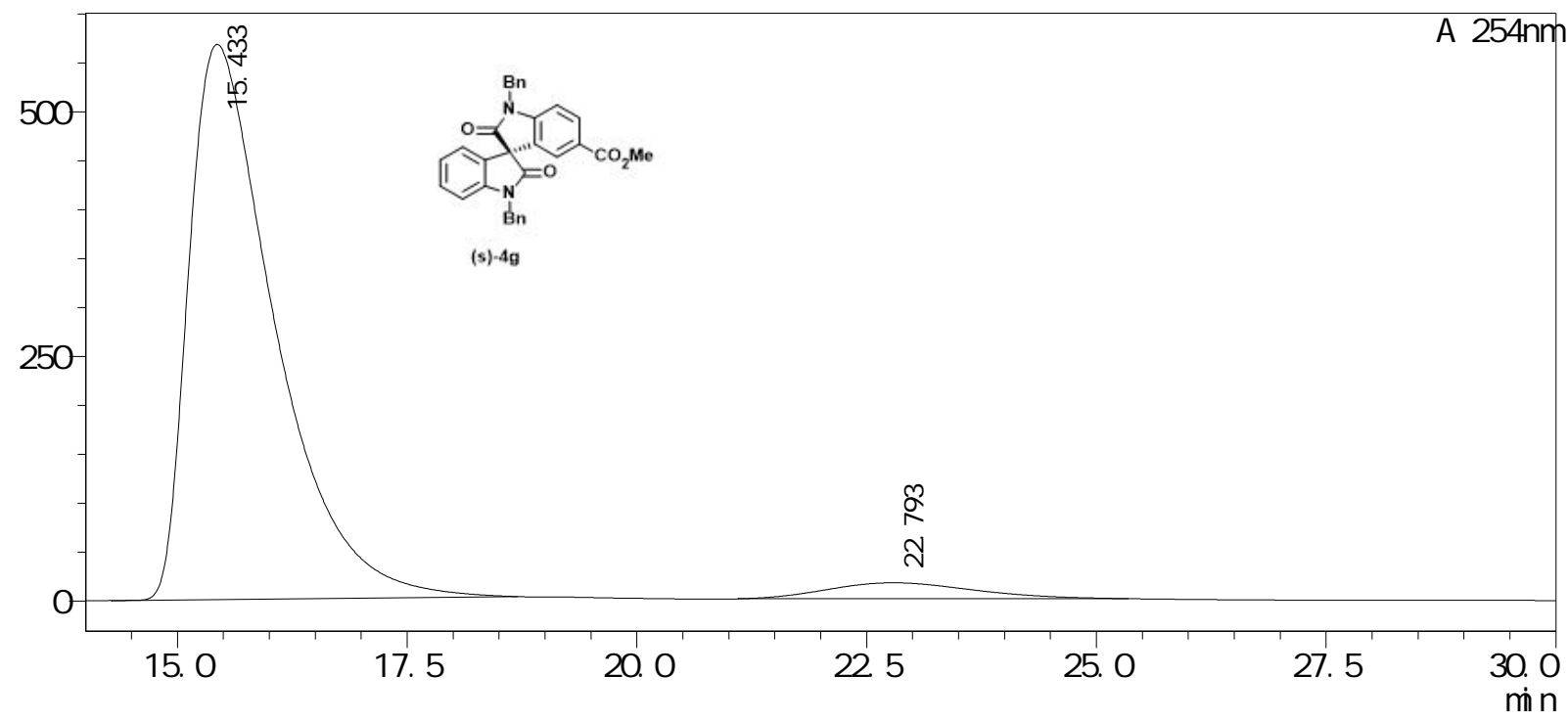

峰表>

检测器A 254nm

留时间

\begin{tabular}{|r|r|r|r|r|r|r|r|}
\hline 峰号 & 保留时间 & \multicolumn{1}{|c|}{ 面积 } & \multicolumn{1}{|c|}{ 高度 } & \multicolumn{1}{|c|}{ 浓度 } & 浓度单位 & 标记 & 化合物名 \\
\hline 1 & 15.433 & 36998758 & 567641 & 95.341 & & $\mathrm{M}$ & \\
\hline 2 & 22.793 & 1807940 & 16368 & 4.659 & & $\mathrm{M}$ & \\
\hline 总计 & & 38806698 & 584008 & & & & \\
\hline
\end{tabular}


 \\ LabSolutions 分析报告}

《样品信息>

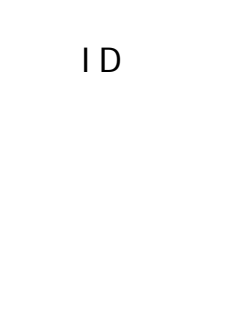

$:$ It

LT201802045-2BI AO OD H 40. I cd

OD H 40\% 100mi n. I cm

1- 1

$1 \mathrm{uL}$

2019/1/10 15: 47: 31

样品类型

: 未知

处理日期

2019/1/10 20: 57: 24

分析者 : Syst em Admi ni st r at or

处理者 : Syst em Admi ni st $r$ at or

<色谱图>

$\mathrm{nV}$

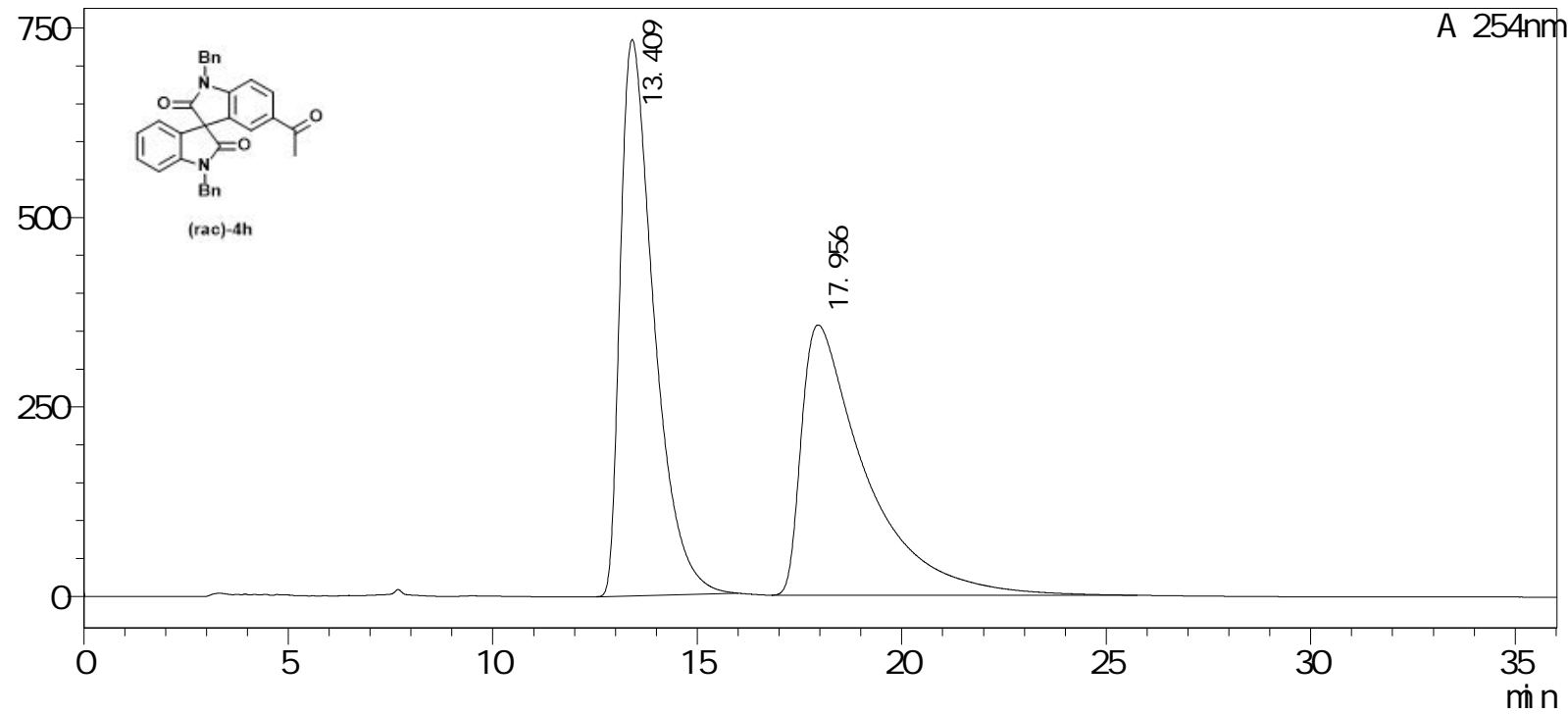

峰表>

检测器A $254 n m$

\begin{tabular}{|r|r|r|r|r|r|r|r|}
\hline 峰号 & 保留时间 & 面积 & 高度 & 浓度 & 浓度单位 & 标记 & 化合物名 \\
\hline 1 & 13.409 & 42033003 & 733996 & 51.827 & & $\mathrm{M}$ & \\
\hline 2 & 17.956 & 39069017 & 356635 & 48.173 & & $\mathrm{M}$ & \\
\hline 总计 & & 81102020 & 1090631 & & & & \\
\hline
\end{tabular}


 \\ LabSolutions 分析报告}

《样品信息>

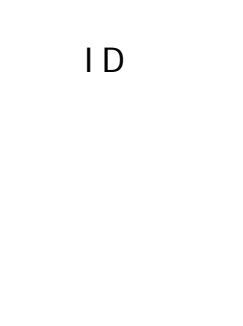

$:$ It

: LT201802055- OD H 40. I cd

OD H 40\% 100m n. I cm

: $1-1$

$1 \mathrm{uL}$

2019/1/15 15: 45: 03

样品类型 ：未知

处理日期

2019/1/15 20: 52: 49

分析者 : Syst em Admi ni st $r$ at or

处理者 : Syst em Admi ni st $r$ at or

<色谱图>

nV

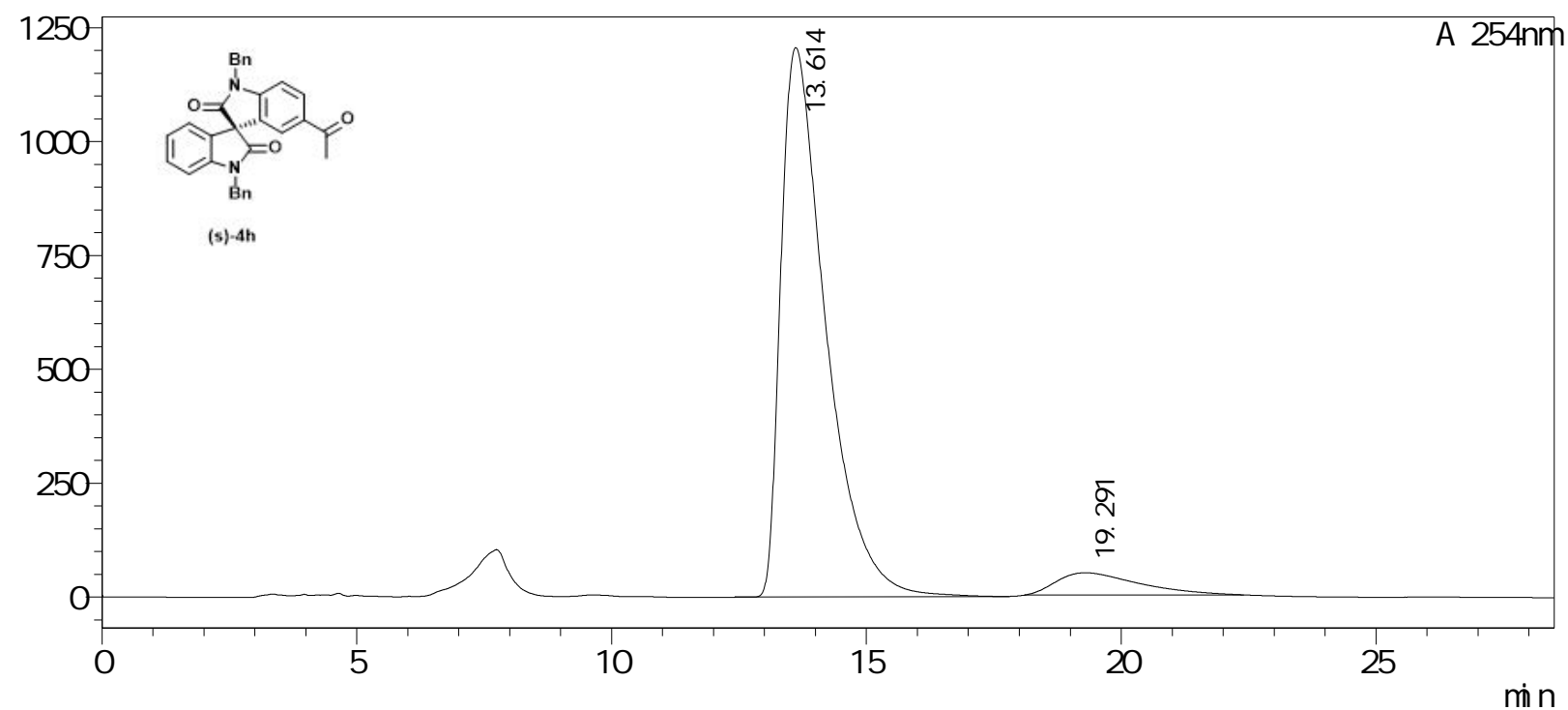

峰表>

检测器A $254 \mathrm{~nm}$

\begin{tabular}{|r|r|r|r|r|r|r|r|}
\hline 峰号 & 保留时间 & \multicolumn{1}{|c|}{ 面积 } & \multicolumn{1}{|c|}{ 高度 } & \multicolumn{1}{|c|}{ 浓度 } & 浓度单位 & 标记 & 化合物名 \\
\hline 1 & 13.614 & 74014635 & 1206000 & 93.064 & & $\mathrm{M}$ & \\
\hline 2 & 19.291 & 5516401 & 48947 & 6.936 & & $\mathrm{M}$ & \\
\hline 总计 & & 79531036 & 1254947 & & & & \\
\hline
\end{tabular}


 \\ LabSolutions 分析报告}

《样品信息>

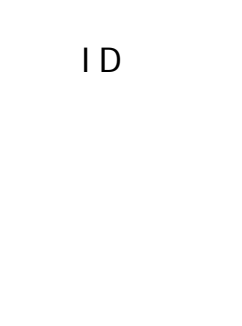

: It

LT201802075. 3 OJ- H 40. I cd

OJ. H $40 \% 60 \mathrm{~min}$. I cm

1- 1

$1 \mathrm{uL}$

样品类型 ：未知

处理日期

2019/1/24 15: 15: 21

2019/1/24 16: $28: 07$

分析者

处理者

: Syst em Admi ni st r at or

: Syst em Admi ni st $r$ at or

<色谱图>

nV

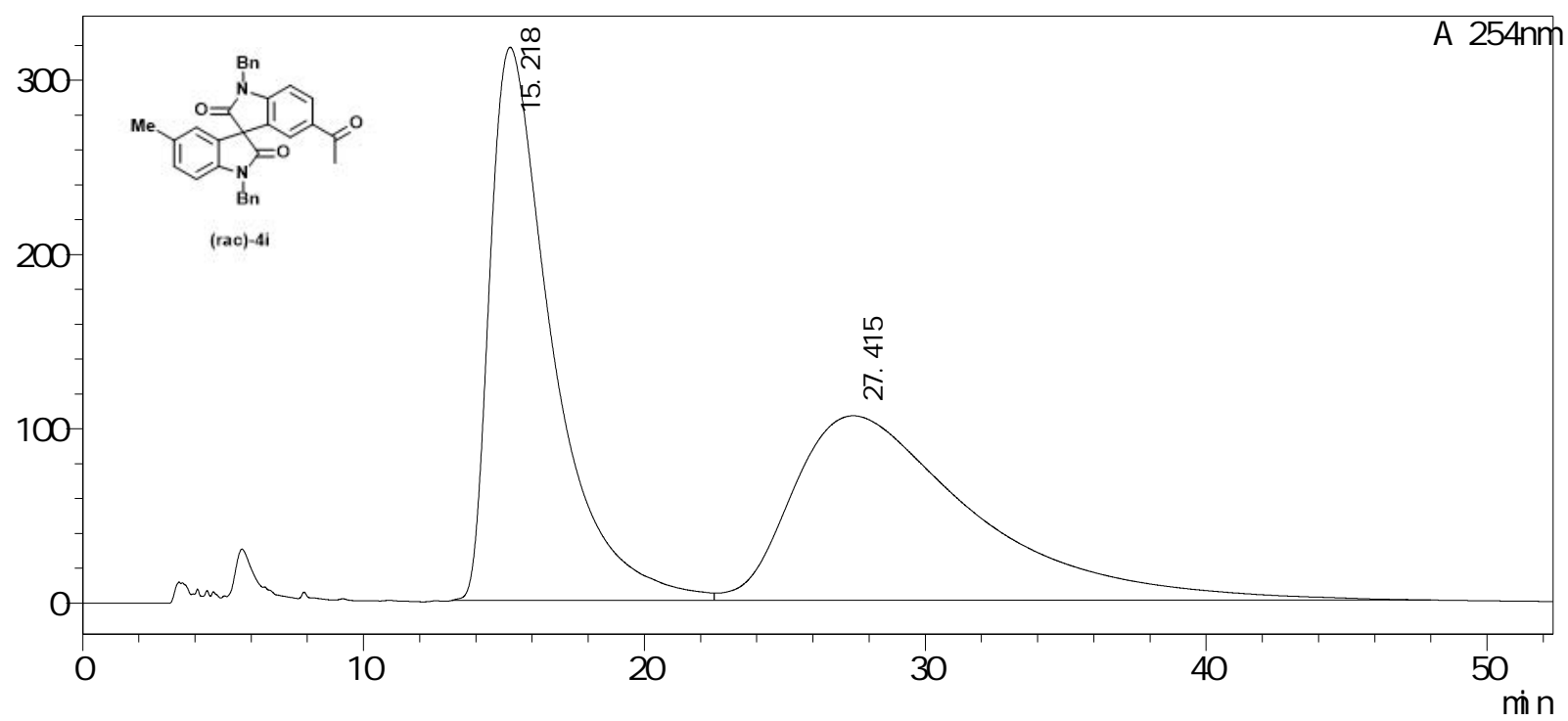

峰表>

检测器A $254 \mathrm{~nm}$

\begin{tabular}{|r|r|r|r|r|r|r|r|}
\hline 峰号 & 保留时间 & 面积 & 高度 & 浓度 & 浓度单位 & 标记 & 化合物名 \\
\hline 1 & 15.218 & 48971429 & 317385 & 50.758 & & $\mathrm{M}$ & \\
\hline 2 & 27.415 & 47508955 & 105882 & 49.242 & & $\mathrm{~V} \mathrm{M}$ & \\
\hline 总计 & & 96480384 & 423267 & & & & \\
\hline
\end{tabular}


 \\ LabSolutions 分析报告}

《样品信息>

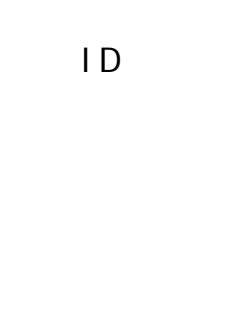

$:$ It

LT201802080. 2- OJ- H 40. I cd

OJ. H $40 \% 60 \mathrm{~min}$. I cm

1- 1

$1 \mathrm{uL}$

样品类型：未知

处理日期

2019/1/24 16: 10: 16

分析者

: 未知

2019/1/24 17: $42: 09$

处理者

<色谱图>

$\mathrm{nV}$

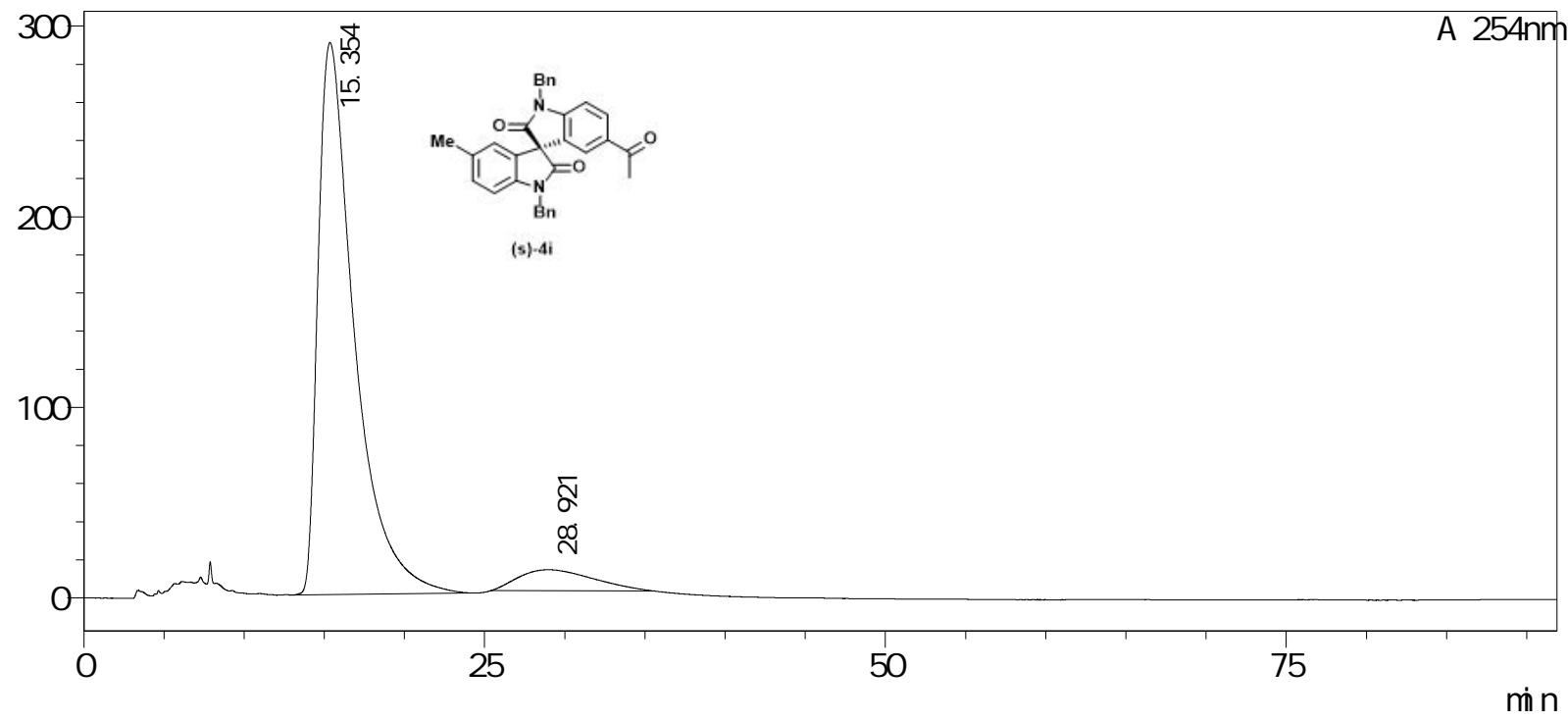

《峰表>

检测器 $\mathrm{A} 254 \mathrm{~nm}$

\begin{tabular}{|r|r|r|r|r|r|r|r|}
\hline 峰号 & 保留时间 & \multicolumn{1}{|c|}{ 面积 } & \multicolumn{1}{|c|}{ 高度 } & \multicolumn{1}{|c|}{ 浓度 } & 浓度单位 & 标记 & 化合物名 \\
\hline 1 & 15.354 & 45616006 & 289629 & 92.696 & & $\mathrm{M}$ & \\
\hline 2 & 28.921 & 3594086 & 11013 & 7.304 & & $\mathrm{M}$ & \\
\hline 总计 & & 49210092 & 300642 & & & & \\
\hline
\end{tabular}

